Schriftenreihe des Instituts für Angewandte Informatik / Automatisierungstechnik Universität Karlsruhe (TH) Band 5

Dipl.-Ing. Jörg Matthes

\title{
Eine neue Methode zur Quellenlokalisierung auf der Basis räumlich verteilter, punktweiser Konzentrationsmessungen
}

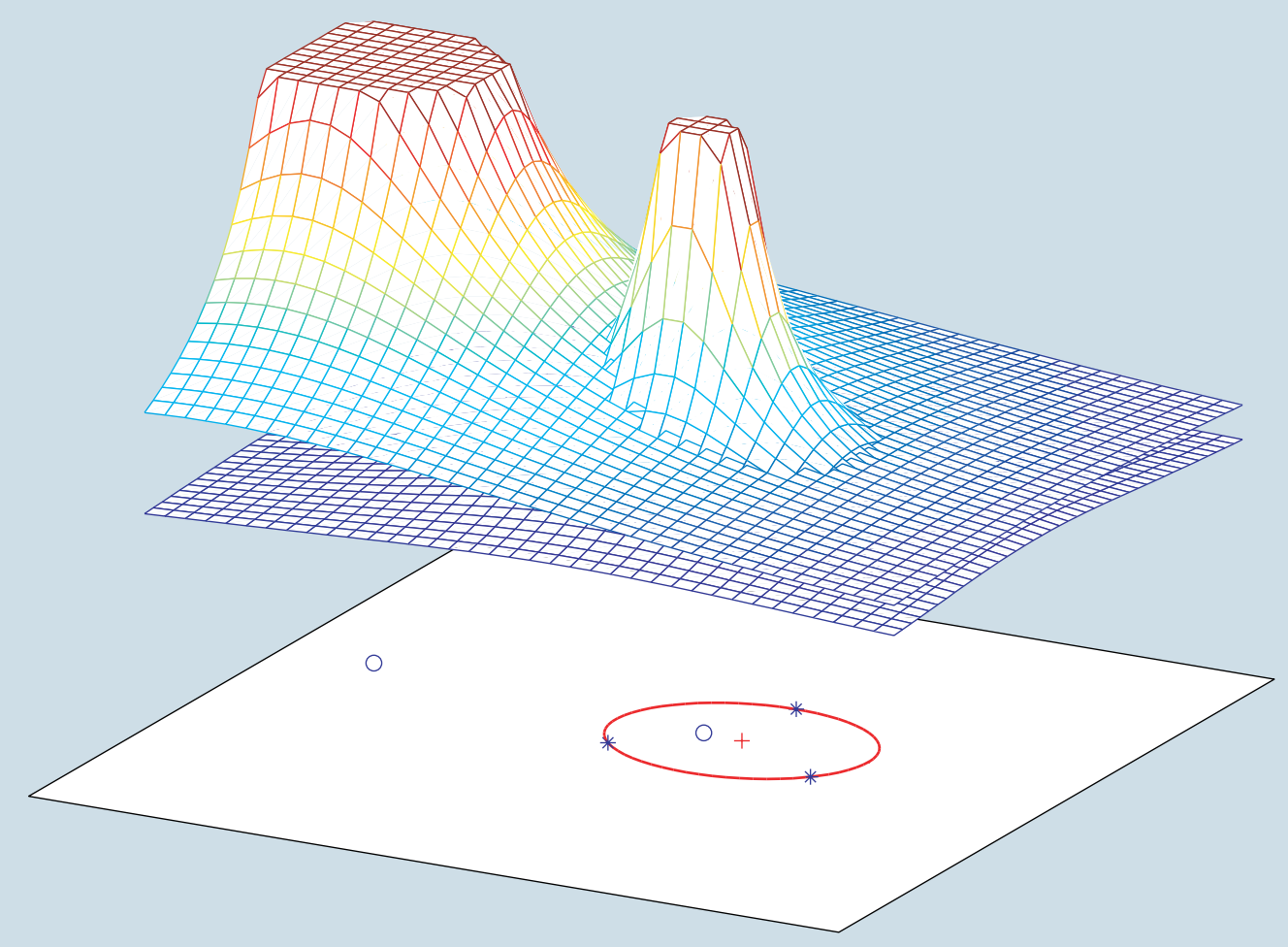


Dissertation, Universität Karlsruhe (TH), Fakultät für Maschinenbau, 2004

\section{Impressum}

Universitätsverlag Karlsruhe c/o Universitätsbibliothek

Straße am Forum 2

D-76131 Karlsruhe

www.uvka.de

(c) Universitätsverlag Karlsruhe 2004

Print on Demand

ISSN 1614-5267

ISBN 3-937300-16-3 


\title{
Eine neue Methode zur Quellenlokalisierung auf der Basis räumlich verteilter, punktweiser Konzentrationsmessungen
}

\author{
Zur Erlangung des akademischen Grades eines \\ Doktors der Ingenieurwissenschaften \\ von der Fakultät für Maschinenbau der \\ Universität Karlsruhe genehmigte \\ DisserTATION \\ von \\ Dipl.-Ing. Jörg Matthes \\ geb. am 30. März 1976 in Dresden
}

Tag der mündlichen Prüfung:

23. Juli 2004

Hauptreferent:

Prof. Dr.-Ing. habil. Georg Bretthauer

Korreferent:

Prof. Dr.-Ing. habil. Jürgen Wernstedt 


\section{Danksagung}

Die vorliegende Dissertation entstand während meiner Tätigkeit als wissenschaftlicher Mitarbeiter am Institut für Angewandte Informatik des Forschungszentrums Karlsruhe. Mein ganz besonderer Dank gilt Herrn Prof. Dr.-Ing. habil. Georg Bretthauer, der die Arbeit ermöglichte und mich durch Diskussionen und kritische Hinweise unterstützte.

Für die Übernahme des Korreferats und wertvolle fachliche Hinweise bin ich zudem Herrn Prof. Dr.-Ing. habil. Jürgen Wernstedt zu Dank verpflichtet.

Weiterhin möchte ich Herrn Dr.-Ing. Hubert B. Keller danken, der als Leiter der Projektgruppe „Intelligente Sensorsysteme“ die Beschäftigung mit der Thematik anregte und begleitete.

Besonders danke ich Herrn Dr.-Ing. Lutz Gröll für die zahlreichen fachlichen Hinweise und Diskussionen sowie für die Bereitstellung eines von ihm entwickelten Algorithmus zur Lösung quadratisch restringierter, linearer Least-squares-Probleme.

Weiterhin bedanke ich mich bei den Mitarbeitern des HGF-Strategiefondsprojekts „Elektronische Mikronasen für Überwachungs- und Regelaufgaben in Gebäuden und Produktionsanlagen" und insbesondere bei Frau Dr.-Ing. Christina Arnold für die tatkräftige Unterstützung bei den Experimenten.

Zudem möchte ich mich bei allen Kollegen und Studenten bedanken, die mich durch Diskussionsbeiträge, Hinweise und Diplomarbeiten unterstützt haben. 


\section{Inhaltsverzeichnis}

$\begin{array}{ll}\text { Wichtige Formelzeichen und Symbole } & \text { IV }\end{array}$

1 Einleitung 1

1.1 Motivation . . . . . . . . . . . . . . . . . 1

1.2 Bestehende Verfahren zur Quellenlokalisierung . . . . . . . . . . . . . 2

1.2 .1 Überblick . . . . . . . . . . . . . . . . . . . . . . . . . . . 2

1.2.2 Quellenlokalisierung mit mobilen Sensoren . . . . . . . . . . . . 3

1.2.3 Ad-hoc-Verfahren zur Quellenlokalisierung mit stationären Sensoren 3

1.2.4 Klassifikationsbasierte Quellenlokalisierung mit stationären Sensoren 6

1.2.5 Modellbasierte Quellenlokalisierung mit stationären Sensoren . . . . 6

1.3 Offene Fragen modellbasierter Verfahren . . . . . . . . . . . . . . 8

1.4 Ziele und Gliederung der Arbeit . . . . . . . . . . . . . . . . . . . . . . 10

2 Modellierung der Ausbreitung von Luftbeimengungen 12

2.1 Diffusions-Advektions-Gleichung . . . . . . . . . . . . . . . . . . . . . . . 12

2.2 Vereinfachung der Ausbreitungsgleichung . . . . . . . . . . . . . . . . . . 14

2.3 Wahl des Quellterms . . . . . . . . . . . . . . . . . . 15

2.4 Lösungen der Diffusions-Advektions-Gleichung . . . . . . . . . . . . . . . . 16

2.4 .1 Greensche Funktion . . . . . . . . . . . . . . . . . . . 16

2.4.2 Diffusion im unendlichen Medium . . . . . . . . . . . . . . . 17

2.4.3 Diffusion-Advektion im unendlichen Medium . . . . . . . . . . . . 21

2.4.4 Lösungen für das halbunendliche Medium . . . . . . . . . . . . . . 22

2.4.5 Lösung und Diskussion für das endliche Medium . . . . . . . . . . . 24

2.5 Lösungen für ein- und zweidimensionale Medien . . . . . . . . . . . . . 26

2.6 Zusammenhang zwischen instantaner und sprungförmiger Quelle . . . . . . 28

2.7 Experimentelle Modellvalidierung . . . . . . . . . . . . . . . . . . . . . . . 29

2.8 Konsequenzen für die Quellenlokalisierung . . . . . . . . . . . . . . . . 31 
3 Ein neues Verfahren zur Quellenlokalisierung bei Diffusion 34

3.1 Schätzung skalierbarer Sensor-Quell-Abstände . . . . . . . . . . . . . 36

3.1.1 Schätzung auf der Basis stationärer Konzentrationen . . . . . . . . 36

3.1.2 Schätzung auf der Basis instationärer Konzentrationen ..... . 37

3.1.3 Bewertung der Schätzverfahren . . . . . . . . . . . . . . . . . . 40

3.2 Ermittlung des Quellorts im ungestörten Fall . . . . . . . . . . . . . . . . 41

3.2.1 Vorbemerkungen . . . . . . . . . . . . . . . . 41

3.2 .2 Linienkonfiguration . . . . . . . . . . . . . . . . . . . . 42

3.2.3 Ebenenkonfiguration . . . . . . . . . . . . . . . . . 43

3.2.4 Raumkonfiguration . . . . . . . . . . . . . . . . . . 49

3.2.5 Halbraumkonfiguration . . . . . . . . . . . . . . . 50

3.2 .6 Allgemeine Lösung . . . . . . . . . . . . . . . . . . . . . . . 51

3.3 Untersuchung der Lösung . . . . . . . . . . . . . . . . . . . 52

3.3.1 Eigenschaften von wahrem und scheinbarem Quellort . . . . . . . 52

3.3.2 Eigenschaften von wahrem und scheinbarem Skalierungsfaktor . . . 57

3.3.3 Konsequenzen für die Sensorplatzierung . . . . . . . . . . . . . 58

3.3.4 Zur Existenz von Lösungen im gestörten Fall . . . . . . . . . . . . . 59

3.4 Ermittlung des Quellorts im gestörten Fall . . . . . . . . . . . . . . . . . 60

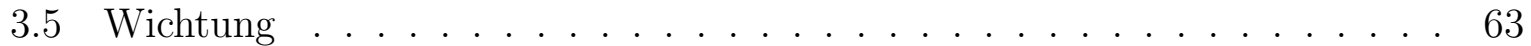

3.6 Ermittlung der Quellintensität . . . . . . . . . . . . . . 65

3.6 .1 Vorbemerkung . . . . . . . . . . . . . . . 65

3.6.2 Ermittlung der Quellintensität mit stationären Konzentrationen . 66

3.6.3 Ermittlung der Quellintensität mit instationären Konzentrationen 67

3.7 Fehler der Quellortschätzung mit stationären Konzentrationen . . . . . . . 67

3.8 Probleme bei ungünstigen Sensorstandorten . . . . . . . . . . . . . 68

3.9 Zusammenfassung . . . . . . . . . . . . . . . . 70

4 Ein neues Verfahren zur Quellenlokalisierung bei Diffusion-Advektion 72

4.1 Schätzung verallgemeinerter skalierbarer Sensor-Quell-Abstände . . . . . . 74

4.2 Quellortschätzung im ungestörten Fall . . . . . . . . . . . . . . . . . 75

4.2.1 Vorbemerkungen . . . . . . . . . . . . . . . . . 75

4.2 .2 Linienkonfiguration . . . . . . . . . . . . . . . . 76

4.2 .3 Ebenenkonfiguration . . . . . . . . . . . . . . . . . 80

4.2.4 Raumkonfiguration . . . . . . . . . . . . . . . . 83

4.2.5 Halbraumkonfiguration . . . . . . . . . . . . . . . . . 84

4.2 .6 Allgemeine Lösung . . . . . . . . . . . . . . . . . . . . . . . . . 84 
4.3 Untersuchung der Lösung . . . . . . . . . . . . . . . . . . . . . . 84

4.3.1 Eigenschaften von wahrem und scheinbarem Quellort . . . . . . . . 84

4.3.2 Konsequenzen für die Sensorplatzierung . . . . . . . . . . . . . . 85

4.3.3 Zur Existenz von Lösungen im gestörten Fall . . . . . . . . . . . . . 88

4.4 Quellortschätzung im gestörten Fall . . . . . . . . . . . . . . . . . . 88

4.5 Wichtung . . . . . . . . . . . . . . . . . . . . . 90

4.6 Fehler der Quellortschätzung mit stationären Konzentrationen . . . . . . . 93

4.7 Zusammenfassung . . . . . . . . . . . . . . . . . . . . . . . . . 94

5 Simulative und praktische Erprobung der neuen Verfahren 96

5.1 Komplexbeispiel Diffusion . . . . . . . . . . . . . . . . . . . . . 96

5.2 Komplexbeispiel Diffusion-Advektion . . . . . . . . . . . . . . . 100

5.3 Praktische Erprobung . . . . . . . . . . . . . . . . . . . . . . 104

6 Zusammenfassung und Ausblick 111

$\begin{array}{lr}\text { A Elektronische Nasen } & 114\end{array}$

$\begin{array}{ll}\text { B Nachweis der Greenschen Funktionen } & 117\end{array}$

C Übergang vom drei- zum zweidimensionalen Medium 121

D Nachweis $\frac{\mathrm{dC}_{\mathrm{S}}(\mathrm{x}, t)}{\mathrm{d} t}=\mathrm{C}_{\mathrm{G}}(\mathrm{x}, t) \cdot q_{0}$

$\begin{array}{ll}\text { Literaturverzeichnis } & 126\end{array}$ 


\section{Wichtige Formelzeichen und Symbole}

\begin{tabular}{|c|c|}
\hline$C(\mathbf{x}, t)$ & Konzentration als Funktion von Ort und Zeit \\
\hline$C_{G}(\mathbf{x}, t)$ & Greensche Funktion bei Diffusion \\
\hline$C_{G, A}(\mathbf{x}, t)$ & Greensche Funktion bei Diffusion und Advektion \\
\hline \multirow[t]{2}{*}{$C_{I}(\mathbf{x}, t)$} & Lsg. der Ausbreitungsgleichung für impulsförmige Intensität bei \\
\hline & Diffusion im unendlichen, dreidimensionalen Medium \\
\hline \multirow[t]{2}{*}{$C_{I, H}(\mathbf{x}, t)$} & Lsg. der Ausbreitungsgleichung für impulsförmige Intensität bei \\
\hline & Diffusion im halbunendlichen, dreidimensionalen Medium \\
\hline \multirow[t]{2}{*}{$C_{I, A}(\mathbf{x}, t)$} & Lsg. der Ausbreitungsgleichung für impulsförmige Intensität bei \\
\hline & Diffusion-Advektion im unendlichen, dreidimensionalen Medium \\
\hline \multirow[t]{2}{*}{$C_{I, A, H}(\mathbf{x}, t)$} & Lsg. der Ausbreitungsgleichung für impulsförmige Intensität bei \\
\hline & Diffusion-Advektion im halbunendlichen, dreidimensionalen Medium \\
\hline \multirow[t]{2}{*}{$C_{I}^{1 D}(\mathbf{x}, t)$} & Lsg. der Ausbreitungsgleichung für impulsförmige Intensität bei \\
\hline & Diffusion im unendlichen, eindimensionalen Medium \\
\hline \multirow[t]{2}{*}{$C_{I, A}^{1 D}(\mathbf{x}, t)$} & Lsg. der Ausbreitungsgleichung für impulsförmige Intensität bei \\
\hline & Diffusion-Advektion im unendlichen, eindimensionalen Medium \\
\hline \multirow[t]{2}{*}{$C_{S}^{1 D}(\mathbf{x}, t)$} & Lsg. der Ausbreitungsgleichung für sprungförmige Intensität bei \\
\hline & Diffusion im unendlichen, eindimensionalen Medium \\
\hline \multirow[t]{2}{*}{$C_{I}^{2 D}(\mathbf{x}, t)$} & Lsg. der Ausbreitungsgleichung für impulsförmige Intensität bei \\
\hline & Diffusion im unendlichen, zweidimensionalen Medium \\
\hline \multirow[t]{2}{*}{$C_{S}^{2 D}(\mathbf{x}, t)$} & Lsg. der Ausbreitungsgleichung für sprungförmige Intensität bei \\
\hline & Diffusion im unendlichen, zweidimensionalen Medium \\
\hline \multirow[t]{2}{*}{$C_{S}(\mathbf{x}, t)$} & Lsg. der Ausbreitungsgleichung für sprungförmige Intensität bei \\
\hline & Diffusion im unendlichen dreidimensionalen Medium \\
\hline \multirow[t]{2}{*}{$C_{S, H}(\mathbf{x}, t)$} & Lsg. der Ausbreitungsgleichung für sprungförmige Intensität bei \\
\hline & Diffusion im halbunendlichen dreidimensionalen Medium \\
\hline \multirow[t]{2}{*}{$C_{S, A}(\mathbf{x}, t)$} & Lsg. der Ausbreitungsgleichung für sprungförmige Intensität bei \\
\hline & Diffusion-Advektion im unendlichen dreidimensionalen Medium \\
\hline \multirow[t]{2}{*}{$C_{S, A, H}(\mathbf{x}, t)$} & Lsg. der Ausbreitungsgleichung für sprungförmige Intensität bei \\
\hline & Diffusion-Advektion im halbunendlichen dreidimensionalen Medium \\
\hline$C_{i}$ & gemessene Konzentration am Sensor $i$ \\
\hline$C_{i, k}$ & gemessene Konzentration am Sensor Sensor $i$ zur Abtastzeit $t_{k}$ \\
\hline$d$ & Abstand zur Quelle; $d=\left\|\mathbf{x}-\mathbf{x}_{0}\right\|_{2}$ \\
\hline$d_{i}$ & Sensor-Quell-Abstand für Sensor $i ; d_{i}=\left\|\mathbf{x}_{i}-\mathbf{x}_{0}\right\|_{2}$ \\
\hline
\end{tabular}




\begin{tabular}{|c|c|}
\hline$\delta(t)$ & Dirac-Funktion \\
\hline $\operatorname{erfc}(b)$ & Komplement der Fehlerfunktion, $\operatorname{erfc}(b)=\frac{2}{\sqrt{\pi}} \int_{b}^{\infty} \exp \left(-\tau^{2}\right) \mathrm{d} \tau$ \\
\hline$\gamma$ & Skalierungsfaktor \\
\hline$g_{i}$ & Wichtungsfaktor für Sensor $i$ \\
\hline$i$ & laufende Indices für Sensoren \\
\hline$J(\theta)$ & Gütewert in Abhängigkeit vom Parametervektor \\
\hline$k$ & diskrete Zeit \\
\hline$K$ & isotroper Diffusionskoeffizient \\
\hline$K_{x}, K_{y}, K_{z}$ & Diffusionskoeffizienten in $\mathrm{x}-, \mathrm{y}-$ und $\mathrm{z}$-Richtung \\
\hline $\boldsymbol{I}$ & Einheitsmatrix \\
\hline$m$ & minimale Anzahl von Sensoren für die Quellenlokalisierung \\
\hline$M_{0}$ & freigesetzte Stoffmenge bei instantanem Quellverlauf \\
\hline$N(\mathbf{A})$ & Nullraum einer Matrix A \\
\hline$n$ & Dimension des Ausbreitungsmediums (Kap. 2); \\
\hline & Dimension des Quellorts (Kap. 3 bis 5) \\
\hline $\mathcal{P}_{i}$ & Menge der Punkte, auf der die Quelle aus Sicht von Sensor $i$ liegt \\
\hline$p$ & Anzahl von Sensoren für die Quellenlokalisierung \\
\hline$q(t)$ & zeitabhängige Quellintensität \\
\hline$q_{0}$ & konstante Quellintensität bei sprungförmigem Quellverlauf \\
\hline$Q(\mathbf{x}, t)$ & Quellterm \\
\hline$R$ & Radius des Sensorumkreises bzw. der Sensorumkugel \\
\hline$R g(\mathbf{A})$ & Rang einer Matrix A \\
\hline$\varrho_{i}$ & skalierbarer Sensor-Quell-Abstand für Sensor $i$ \\
\hline$r_{S}$ & Radius eines Schnittkreises \\
\hline$t$ & Zeit (kontinuierlich) \\
\hline$t_{0}$ & Wirkungsbeginn der Emissionsquelle (kontinuierlich) \\
\hline$\theta$ & Parametervektor \\
\hline $\boldsymbol{\theta}_{i: j}$ & Teil des Parametervektors, \\
\hline & der sich aus den Komponenten $i$ bis $j$ zusammensetzt \\
\hline$v_{x}, v_{y}, v_{z}$ & Advektionsgeschwindigkeiten in $\mathrm{x}-, \mathrm{y}$ - und z-Richtung \\
\hline$W_{0}(z)$ & Lambert-W-Funktion, Inverse der Funktion $f(w)=w \mathrm{e}^{w}=z$ \\
\hline$w_{i}$ & Wichtungsfaktor für Sensor $i$ \\
\hline $\mathbf{x}_{M}$ & Mittelpunkt des Sensorumkreises bzw. der Sensorumkugel \\
\hline $\mathbf{x}_{S}$ & Mittelpunkt eines Schnittkreises; $\mathbf{x}_{S}=\left(x_{S}, y_{S}\right)^{T}$ \\
\hline $\mathrm{x}$ & Ortsvektor $\mathbf{x}=(x, y, z)^{T}$ \\
\hline
\end{tabular}


$\mathbf{x}_{0}$

$\mathbf{x}_{i}$

$\|\cdot\|_{2}$

$\mathbf{x}^{T}, \mathbf{A}^{T}$

$\mathrm{A}^{-1}$

$\mathbf{A}^{+}$

$1(t)$

Quellort $\mathbf{x}_{0}=\left(x_{0}, y_{0}, z_{0}\right)^{T}$

Standort $i$-ten Sensors $\mathbf{x}_{i}=\left(x_{i}, y_{i}, z_{i}\right)^{T}$

Euklidische Vektornorm

transponierter Vektor bzw. transponierte Matrix

Inverse einer Matrix

Moore-Penrose-Inverse einer Matrix

Sprungfunktion 


\section{Einleitung}

\subsection{Motivation}

In zahlreichen Anwendungen, z. B.

- Leckageerkennung in Gefahrenstofflagern [2, 3],

- Überwachung der Luftqualität auf Deponien [54] und

- Ortung von Landminen [55],

besteht die Aufgabe darin, die Emissionsquelle einer bestimmten Luftbeimengung zu lokalisieren. Bevorzugt geschieht dies über räumlich verteilte Online-Konzentrationsmessungen. Solche Konzentrationsmessungen wurden in den letzten Jahren durch so genannte Elektronische Nasen [28, 29, 30, 34, 37, 57, 63, 93, 103] (Anhang A) möglich, die durch die Anwendung der Mikrosystemtechnik den Aufbau preiswerter Sensornetzwerke erlauben. Aus diesem Grund gewinnt auch die Quellenlokalisierung auf der Basis räumlich verteilter Konzentrationsmessungen in letzter Zeit an Bedeutung.

Erste Anwendungen räumlich verteilter Sensornetze zielten zunächst auf eine Emissionsdetektion. Eine Emission wird dann detektiert, wenn der Konzentrationsschwellwert für den betreffenden Stoff an einem oder an mehreren Sensoren überschritten wird. Aus der Lage dieser Sensoren lässt sich jedoch, abhängig vom Abstand der Sensoren untereinander, nur eine sehr grobe Aussage über den Quellort treffen. Der Quellort kann aber weitaus genauer oder mit einer geringeren Anzahl von Sensoren ermittelt werden, wenn die gemessenen Konzentrationen durch geeignete Verfahren zur Lokalisierung der Emissionsquelle genutzt werden.

In der vorliegenden Arbeit werden zunächst bestehende Verfahren zur Quellenlokalisierung untersucht und deren Schwachpunkte herausgearbeitet. Ausgehend von einem neuen, geometrischen Zugang werden auf der Basis analytischer Lösungen der Diffusions-AdvektionsGleichung neue, zweistufige Verfahren entwickelt, die diese Schwächen umgehen und zudem tiefere Untersuchungen des Quellenlokalisierungsproblems zulassen. 
Da die Diffusions-Advektionsgleichung (parabolische partielle Differenzialgleichungen zweiter Ordnung) nicht nur für die Ausbreitung von Luftbeimengungen in gasförmigen Medien, sondern grundsätzlich auch für

- die Ausbreitung von Schadstoffen in flüssigen sowie porösen Medien (z. B. Grundwasser) [26, 60, 61, 68, 107],

- die Ausbreitung von Wärme in homogenen Medien [18],

- das Verhalten der Schallintensität bei der räumlichen Schallausbreitung [76, 88, 111]

gilt, sind die in dieser Arbeit entwickelten Verfahren und Erkenntnisse zur Quellenlokalisierung prinzipiell auch auf derartige Anwendungsfelder übertragbar.

\subsection{Bestehende Verfahren zur Quellenlokalisierung}

\subsection{1 Überblick}

Abbildung 1.1 gibt eine Übersicht über die bestehenden Verfahren zur Quellenlokalisierung. In den nachfolgenden Abschnitten werden die Prinzipien sowie die Vor- und Nachteile der gezeigten Verfahren herausgearbeitet.

\section{Quellenlokalisierung}

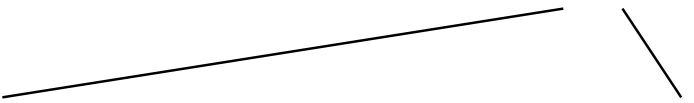

mit mobilen Sensoren

mit stationären Sensoren

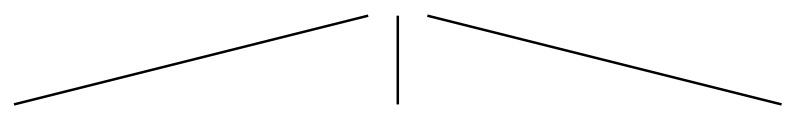

Ad-hoc- modellbasierte klassifikationsbasierte

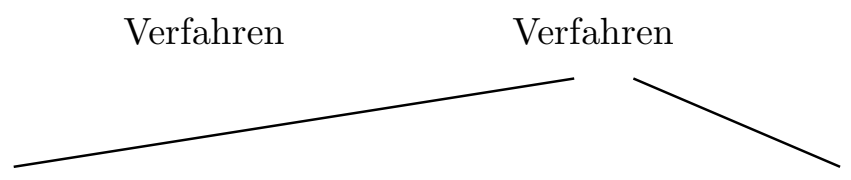

Verfahren

mit numerischen Ausbreitungsmodellen

(Finite Differenzen, Finite Elemente) mit analytischen Ausbreitungsmodellen

(geschlossene Lösungen der

Ausbreitungsgleichung)

Abb. 1.1: Überblick über bestehende Verfahren zur Quellenlokalisierung. 


\subsubsection{Quellenlokalisierung mit mobilen Sensoren}

Die Verfahren zur Quellenlokalisierung mit mobilen Sensoren [24, 48, 51, 52, 64, 65, 95, 96] arbeiten meist nur mit einem Sensor, der durch einen autonomen Roboter bewegt wird. Anhand der gemessenen Konzentration entscheidet der Roboter mittels einer Suchstrategie, in welche Richtung er als nächstes fährt, um sich dem Ort maximaler Konzentration und damit der Quelle zu nähern. Dies wird solange durchgeführt, bis der Roboter den Quellort erreicht. Im industriellen Umfeld ist es jedoch aus technischen und sicherheitstechnischen Gründen oft unmöglich, autonome Roboter frei im Überwachungsgebiet umherfahren zu lassen. Zudem ist ein mobiler Sensor nicht in der Lage, den Quellort einer instantanen (kurzzeitig wirkenden, impulsförmigen) Quelle zu finden, da sich in diesem Fall der Ort maximaler Konzentration mit dem Strömungsfeld bewegt. Der Roboter wird dem Ort maximaler Konzentration bestenfalls hinterherfahren, nicht aber den Quellort finden (Beispiel 1.1).

Beispiel 1.1 Am Quellort $\mathbf{x}_{0}=(5 \mathrm{~m}, 10 \mathrm{~m})^{T}$ wirkt zum Zeitpunkt $t=0$ s eine punktförmige Quelle mit instantaner Quellintensität. Die Ausbreitung erfolgt durch Diffusion und durch Advektion (Strömung) in x-Richtung. In Abb. 1.2 ist die daraus resultierende Konzentrationsverteilung für vier verschiedene Zeitpunkte dargestellt (Linien gleicher Konzentration). Ein mobiler Sensor, der zum Zeitpunkt $t=0$ s die Position $\mathbf{x}_{S}=(10 \mathrm{~m}, 5 \mathrm{~m})^{T}$ besitzt, versucht die Quelle zu orten, indem er sich immer in Richtung maximaler Konzentration bewegt. Das Bild zeigt die Position des Sensors zum jeweiligen Zeitpunkt und die von ihm bis dahin durchfahrene Bahn. Es ist zu erkennen, dass der mobile Sensor den Quellort nicht findet, sondern lediglich dem Punkt maximaler Konzentration folgt.

\subsubsection{Ad-hoc-Verfahren zur Quellenlokalisierung mit stationären Sensoren}

Ad-hoc-Verfahren basieren meist auf einem intuitiven Verständnis der Ausbreitungsvorgänge. Sie nutzen vorwiegend diskrete Informationen, wie z. B. die nach dem Emissionsbeginn verstrichene Zeit bis zum Erreichen von Konzentrationsschwell- oder -maximalwerten.

Ein in [81] vorgeschlagenes Verfahren stützt sich beispielsweise darauf, dass im Fall reiner Diffusion die Zeitspanne zwischen Beginn der Emission und Erreichen eines bestimmten Konzentrationsschwellwerts von der Entfernung des Sensors zur Quelle abhängt. Schwierigkeiten bereitet dabei zum einen die unbekannte Startzeit der Emission. Zum anderen 

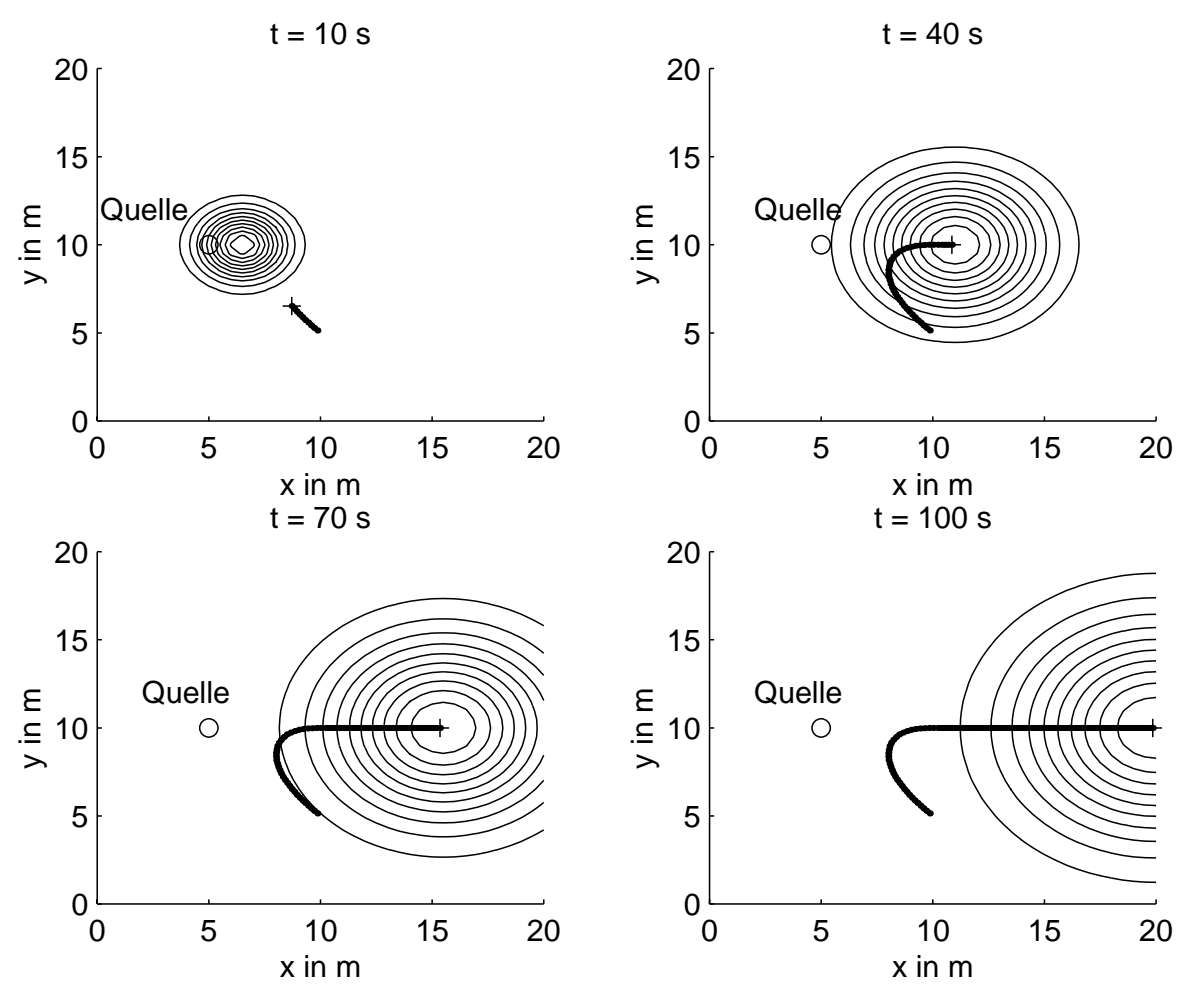

Abb. 1.2: Problem der Quellortung mit einem mobilen Sensor bei instantaner Quelle.

ist die betrachtete Zeitspanne zusätzlich von der Quellintensität (die i. Allg. unbekannt ist) abhängig, was anhand von Beispiel 1.2 verdeutlicht wird.

Beispiel 1.2 Auf der Basis der Konzentrationsmessungen $C_{i, k}$ von vier Sensoren $S_{1}, S_{2}$, $S_{3}, S_{4}$ mit den Positionen $\mathbf{x}_{1}=(0 \mathrm{~m}, 0 \mathrm{~m})^{T}, \mathbf{x}_{2}=(20 \mathrm{~m}, 0 \mathrm{~m})^{T}, \mathbf{x}_{3}=(10 \mathrm{~m}, 10 \mathrm{~m})^{T}$ und $\mathbf{x}_{4}=(10 \mathrm{~m}, 20 \mathrm{~m})^{T}$ zu den Zeitpunkten $t_{k}(k=1, \ldots, N)$ soll eine punktförmige Quelle bei $\mathbf{x}_{0}=(5 \mathrm{~m}, 10 \mathrm{~m})^{T}$ lokalisiert werden $($ Abb. 1.3, links). Es wird angenommen, dass die Quellintensität zum Zeitpunkt $t_{0}=0$ s sprunghaft den konstanten Wert $q_{0}=100 \mathrm{mg} / \mathrm{s}$ annimmt. Die sich ergebenden zeitlichen Verläufe der Konzentrationen an den einzelnen Sensoren zeigt Abb. 1.3 (rechts). Die Sensoren 1 und 4 besitzen den gleichen Abstand zur Quelle und damit den gleichen Konzentrationsverlauf.

Abbildung 1.4 zeigt für jeden Sensor die Zeit $t_{S}$, die an jedem Sensor bis zum Erreichen einer Konzentration von $C_{i}=0.5 \mathrm{mg} / \mathrm{m}^{3}$ (Schwellwert) verstreicht, in Abhängigkeit von der Quellintensität $q_{0}$.

Die Abbildung lässt erkennen, dass die Zeitspanne $t_{S}$ nichtlinear von $q_{0}$ abhängt. Die nichtlineare Abhängigkeit ist zudem vom Abstand des jeweiligen Sensors zur Quelle geprägt und bereits für den gezeigten einfachen Fall reiner Diffusion nicht separabel. Dies stellt den wesentlichen Nachteil schwellwertbasierter Verfahren dar. 

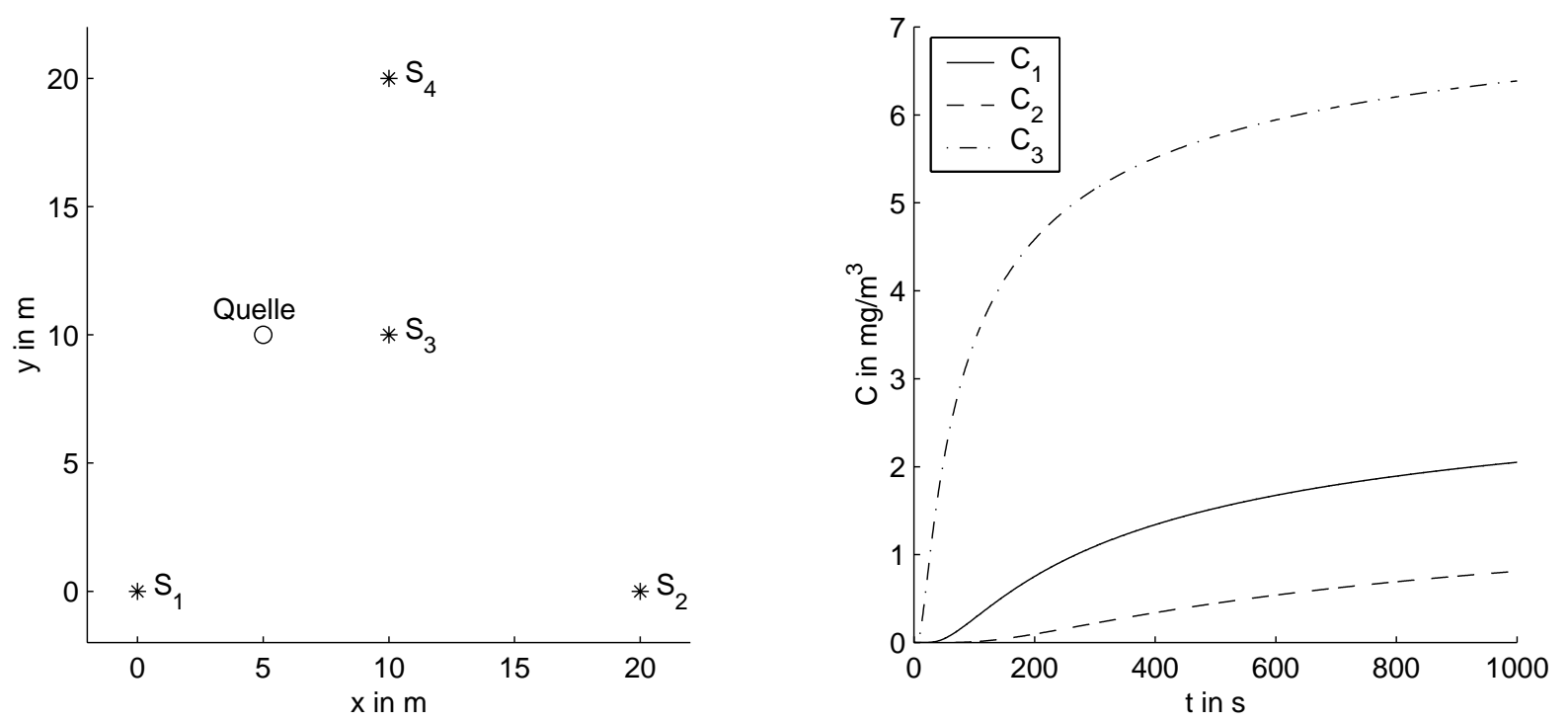

Abb. 1.3: Anordnung von Sensoren und Quelle (links). Simulierte Konzentrationsverläufe für den Fall reiner Diffusion (rechts). (Die Sensoren 1 und 4 besitzen den gleichen Abstand zur Quelle und damit den gleichen Konzentrationsverlauf.)

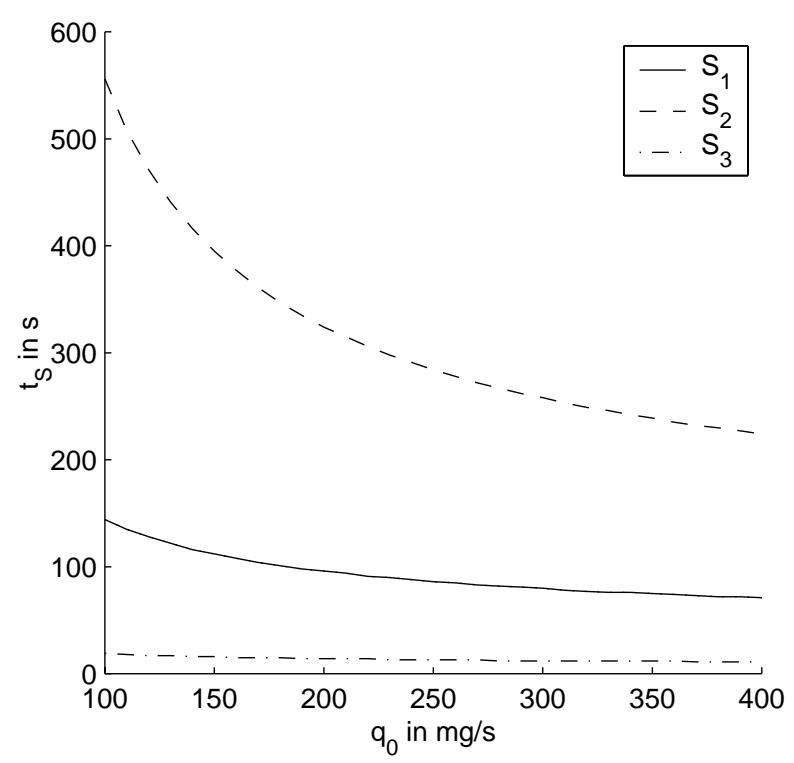

Abb. 1.4: Abhängigkeit der Zeitspanne $t_{S}$ zwischen Emissionsbeginn und Erreichen des Konzentrationsschwellwerts $C=0.5 \mathrm{mg} / \mathrm{m}^{3}$ von der Quellintensität $q_{0}$ für jeden Sensor $i$. (Die Sensoren $S_{1}$ und $S_{4}$ besitzen den gleichen Abstand zur Quelle und damit den gleichen Verlauf für $t_{S}$.)

Ein weiteres Ad-hoc-Verfahren wird in [62] für den Fall eindimensionaler Ausbreitung durch Diffusion-Advektion (konstante Strömung) in einem Gebäudeflur untersucht. Eine instantan wirkende Emissionsquelle ist dabei mit vier im Flur verteilten Sensoren zu lokalisieren. Durch die Bewegung der Emissionswolke ergibt sich an jedem Sensor in Strömungsrichtung ein zeitlicher Konzentrationsverlauf, der ein Maximum aufweist. Die Zeitpunkte, an denen an jedem Sensor die maximale Konzentration gemessen wird, sollen für die Quel- 
lenlokalisierung genutzt werden. Aufgrund der Advektion bewegt sich der Ort maximaler Konzentration mit konstanter Strömungsgeschwindigkeit durch den Flur. Daraus kann vermutet werden, dass die Zeitpunkte maximaler Konzentration an den einzelnen Sensoren linear von den Abständen zwischen den Sensoren abhängen. Die Untersuchungen in [62] zeigen jedoch, dass dies nur für große Strömungsgeschwindigkeiten gilt. Bei kleinen Strömungsgeschwindigkeiten bewirkt die Diffusion ein zeitliches Absinken des räumlichen Konzentrationsprofils und damit eine nichtlineare Verschiebung der Zeitpunkte maximaler Konzentration an den Sensoren. Folglich scheitern maximalwertbasierte Verfahren an den gleichen Problemen wie die schwellwertbasierten Verfahren.

\subsubsection{Klassifikationsbasierte Quellenlokalisierung mit stationären Sensoren}

Bei den klassifikationsbasierten Verfahren werden Konzentrationsverläufe (Referenzmuster) an den Sensoren bei Wirken verschiedener zu klassifizierender Emissionsquellen simulativ oder experimentell bestimmt und in der Überwachungsphase klassifiziert [3, 70]. Es wird also davon ausgegangen, dass alle potenziellen Quellorte a priori bekannt sind, was in vielen Anwendungen nicht erfüllt ist. Klassifikationsbasierte Verfahren besitzen zudem Probleme bei der Behandlung der unbekannten Parameter Quellintensität und -startzeitpunkt. Ein vom Autor in [70] vorgestelltes Klassifikationsverfahren umgeht die letztgenannten Nachteile durch einen Prokrustes-Ansatz.

\subsubsection{Modellbasierte Quellenlokalisierung mit stationären Sensoren}

Modellbasierte Verfahren basieren auf einem Ausbreitungsmodell, das den Quellort, die Quellintensität und den Startzeitpunkt der Quelle als unbekannte Parameter enthält. Das Problem des Ermittelns solcher Parameter anhand gemessener Modellausgangsgrößen (hier Konzentrationen) wird in der Mathematik als inverses Problem bezeichnet [40]. Das hier betrachtete Quellenlokalisierungsproblem ist dadurch gekennzeichnet, dass völlig verschiedene Quellparameter zu gleichen oder ähnlichen Konzentrationsmessungen führen können. In solchen Fällen wird von schlecht gestellten inversen Problemen (engl.: ill-posed inverse problems) gesprochen. Diese Eigenschaft des Quellenlokalisierungsproblems stellt die wesentliche Herausforderung für modellbasierte Verfahren dar. 


\section{Verfahren mit numerischen Modellen}

Die Verfahren mit numerischen Modellen arbeiten mit einem stark vereinfachten Zustandsraummodell, das aus einer örtlichen Diskretisierung des Ausbreitungsgebiets resultiert. Diese Vereinfachung zieht nach sich, dass sich Quell- und Sensorstandorte nur in den Gitterpunkten des Diskretisierungsgitters befinden können. Mit einem Beobachteransatz wird in der Überwachungsphase versucht, die unbekannten Eingangsgrößen (Quellort, -intensität und -startzeit) aus den gemessenen Konzentrationen zu bestimmen $[2,3]$.

Der wesentliche Vorteil dieser Verfahren liegt in der Fähigkeit der verwendeten numerischen Modelle, komplizierte Ausbreitungsprobleme (verwinkelte Räume, ortsabhängige Advektions- und Diffusionsparameter usw.) zu berücksichtigen.

Demgegenüber stehen folgende Nachteile:

- Für jeden Gitterpunkt des Diskretisierungsgitters müssen alle Ausbreitungsparameter vorab identifiziert werden. Je nach Diskretisierungsweite sind auf der Basis weniger Sensoren bis zu mehrere Tausend Parameter zu schätzen.

- Bei praktisch sinnvoller Diskretisierungsweite und Sensoranzahl ist die Zahl der Zustandsgrößen, die durch den Beobachter ermittelt werden müssen, wesentlich größer als die Anzahl der gemessenen Zustandsgrößen.

- Eine Analyse des Quellenlokalisierungsproblems beispielsweise hinsichtlich der Existenz und Eindeutigkeit von Lösungen ist mit numerischen Modellen de facto nicht möglich.

\section{Verfahren mit analytischen Modellen}

Verfahren mit analytischen Modellen [55, 59, 83, 84] nutzen analytische Lösungen der Ausbreitungsgleichung (Diffusions-Advektions-Gleichung) und formulieren auf der Basis des Ausgangsfehlers ein einstufiges, nichtlineares Parameterschätzproblem für die gesuchten Größen Quellort, -intensität und -startzeit.

Bei der Verwendung von analytischen Modellen müssen für den praktischen Einsatz vereinfachende Annahmen getroffen werden, damit geschlossene Lösungen für die Ausbreitungsgleichung existieren. Diesem Nachteil stehen aber wesentliche Vorteile gegenüber:

- Das Ausbreitungsmodell ist übersichtlich und enthält wenige zu identifizierende Ausbreitungsparameter. (Im einfachsten Fall ist nur der turbulente Diffusionskoeffizient vorab zu ermitteln). 
- Bei der Lokalisierung müssen nur die Quellparameter (und keine weiteren Zustandsgrößen) geschätzt werden.

- Die geschlossenen Lösungen der Ausbreitungsgleichung erlauben eine theoretische Analyse des Quellenlokalisierungsproblems hinsichtlich der Existenz und Eindeutigkeit von Lösungen sowie hinsichtlich geeigneter Sensorstandorte. Die daraus gewonnenen Aussagen lassen sich prinzipiell auf die Verfahren mit numerischen Modellen übertragen.

\subsection{Offene Fragen modellbasierter Verfahren}

Der vorangegangene Abschnitt zeigte, dass die Verfahren mit analytischen Modellen wesentliche Vorteile besitzen. Jedoch verhindern numerische Schwierigkeiten, die beim iterativen Lösen des nichtlinearen Parameterschätzproblems auftreten, den Online-Einsatz solcher Verfahren. Diese Schwierigkeiten werden nachfolgend erläutert.

Mit einer analytischen Lösung der Ausbreitungsgleichung lassen sich für jeden beliebigen Quellort $\mathbf{x}_{0}=\left(x_{0}, y_{0}\right)^{T}$ Konzentrationswerte $C\left(\mathbf{x}_{i}, t_{k} ; \mathbf{x}_{0}\right)$ für jeden Sensor $i(i=1, \ldots, p)$ mit dem Standort $\mathbf{x}_{i}$ berechnen. Der gesuchte Quellort $\mathbf{x}_{0}$ lässt sich durch Lösen des einstufigen Parameterschätzproblems

$$
J\left(x_{0}, y_{0}\right)=\sum_{i=1}^{p} \sum_{k=1}^{N_{i}}\left(C_{i, k}-C\left(\mathbf{x}_{i}, t_{i, k}, \mathbf{x}_{0}\right)\right)^{2} \stackrel{!}{=} \operatorname{Min}_{\mathbf{x}_{0}}
$$

finden, wenn vereinfachend Quellintensität, Diffusionskoeffizienten sowie Startzeitpunkt der Quelle als bekannt vorausgesetzt werden. In 1.1 bezeichnen $p$ die Anzahl der Sensoren und $C_{i, k}$ den Konzentrationsmesswert von Sensor $i$ zum Zeitpunkt $t_{i, k}\left(k=1, \ldots, N_{i}\right)$. Die in (1.1) enthaltene analytische Lösung der Ausbreitungsgleichung ist parameternichtlinear. Für das Lösen kommen daher Gradientenverfahren zum Einsatz, die in Abhängigkeit von den Startparametern in Nebenminima oder Sattelpunkten abbrechen und damit falsche Lösungen liefern können. Abbildung 1.5 zeigt das Gütegebirge für $J\left(x_{0}, y_{0}\right)$, das sich für die Sensor-Quell-Konfiguration gemäß Beispiel 1.2 ergibt.

An den Sensorstandorten bilden sich im Gütegebirge Maxima. Für die Quellenlokalisierung sind die Minima relevant. Es ist zu erkennen, dass neben dem globalen Minimum im wahren Quellort $\mathbf{x}_{0}=(5 \mathrm{~m}, 10 \mathrm{~m})^{T}$ noch ein weiteres Nebenminimum bei $\mathbf{x}_{0} \approx(15 \mathrm{~m}, 11 \mathrm{~m})^{T}$ existiert. In diesem bricht ein Gradientenverfahren ab und liefert damit eine falsche Lösung. Werden weitere Sensoren hinzugefügt, entstehen weitere lokale Maxima im Gütegebirge, die die Suche nach dem globalen Minimum nochmals erschweren. 


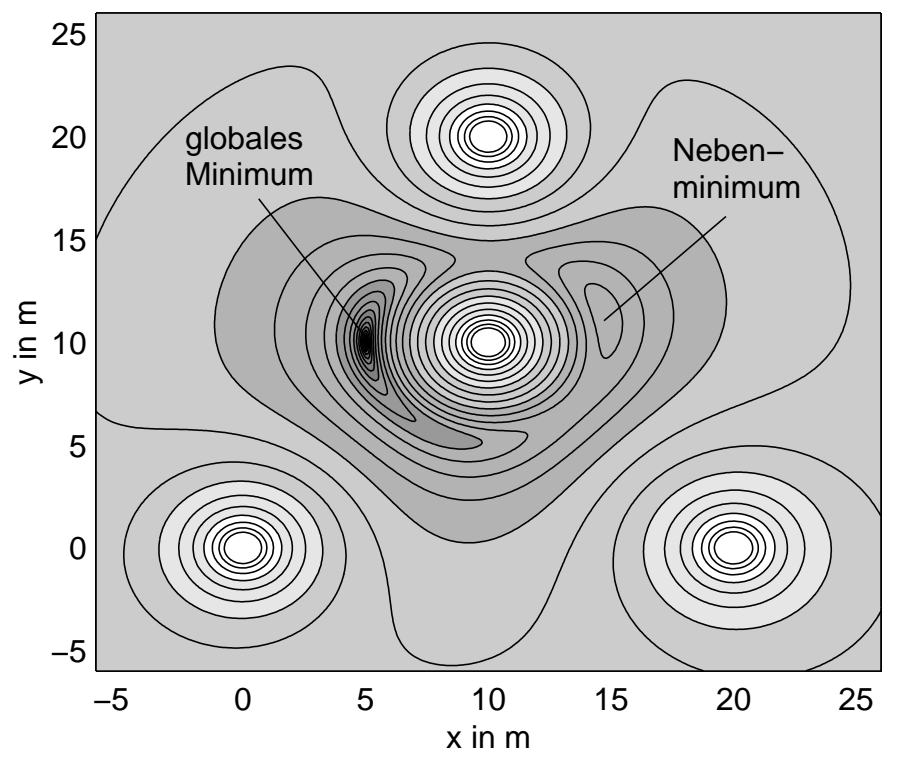

Abb. 1.5: Gütegebirge des Ausgangsfehlers $J\left(x_{0}, y_{0}\right)$ (logarithmisch) in Abhängigkeit vom gewählten Quellort $\mathbf{x}_{0}$. (Dunkle Flächen markieren kleine Werte für $J\left(x_{0}, y_{0}\right)$.)

Dieses Beispiel zeigt, dass einstufige, ausgangsfehlerbasierte Verfahren bereits im einfachsten ungestörten Fall bei Kenntnis der wahren Werte für Quellintensität, Diffusionskoeffizient und Startzeitpunkt der Quelle Probleme bei der Quellenlokalisierung haben. Zusätzlich zu den Nebenminima, die zu falschen Lösungen für den Quellort führen können, führt die Ausprägung des Gütegebirges mit engen Tälern zu einem schlechten Konvergenzverhalten der Gradientenverfahren. Die Situation verschlechtert sich weiter, wenn alle Quellund Ausbreitungsparameter unbekannt sind und sich die Zahl der zu schätzenden Parameter erhöht. Das Hinzufügen von weiteren Sensoren liefert weitere lokale Maxima und damit weitere Täler und lokale Minima im Gütegebirge. Eine Vergrößerung der Sensoranzahl führt damit überraschend zu einer Verschlechterung des Gütegebirges hinsichtlich der Anwendung von Gradientenverfahren und damit zu größeren Schwierigkeiten bei der Suche des globalen Minimums.

Die für den einfachen ungestörten Fall mit reiner Diffusion gezeigten Probleme bleiben prinzipiell auch bei der Betrachtung komplizierterer Fälle (Hinzunahme von Advektion, inhomogene Ausbreitungsmedien usw.), aber auch bei der Anwendung von klassifikationsbasierten Verfahren sowie Verfahren mit numerischen Modellen erhalten oder verschärfen sich sogar. Aus diesem Grund ist es sinnvoll, die vermeintlich einfachen Fälle anhand analytischer Modelle detailliert zu untersuchen. Ferner ist zu prüfen, inwieweit beherrschbare analytische Modelle für praktische Anwendungen geeignet sind. 


\subsection{Ziele und Gliederung der Arbeit}

Ziel der vorliegenden Arbeit ist es, die genannten Probleme bei der Quellenlokalisierung auf der Basis analytischer Ausbreitungsmodelle durch einen neuen, geometrischen Zugang $[36,71,72,73]$ zu umgehen. Die Grundidee des geometrischen Zugangs besteht darin,

- im ersten Schritt für jeden Sensor anhand der gemessenen Konzentrationen die Menge aller Punkte $\mathcal{P}_{i}$ zu bestimmen, auf der sich aus der Sicht des einzelnen Sensors $i$ die Quelle befinden kann und

- im zweiten Schritt durch Bestimmen einer Schnittmenge aller $\mathcal{P}_{i}$ den Quellort zu ermitteln.

In der Arbeit soll der geometrische Zugang dazu genutzt werden, neue Verfahren zur Lokalisierung von Emissionsquellen zu entwickeln. Für die praktische Erprobung der Verfahren werden die Konzentrationsmessungen durch räumlich verteilte, stationäre Elektronische Nasen realisiert. Der geometrische Zugang soll weiterhin dazu dienen, neue Einblicke in das Quellenlokalisierungsproblem hinsichtlich der Existenz und Eindeutigkeit von Lösungen sowie hinsichtlich einer geeigneten Platzierung der Sensoren zu gewinnen.

Die den Verfahren zugrunde liegenden Ausbreitungsmodelle berücksichtigen zum einen turbulente Diffusion ohne Advektion und zum anderen turbulente Diffusion mit homogener Advektion (Diffusion-Advektion). Die Untersuchungen beschränken sich auf den Fall einer punktförmigen Quelle.

Kapitel 2 beschäftigt sich mit der theoretischen Modellierung der Ausbreitung von Luftbeimengungen auf der Basis der Advektions-Diffusions-Gleichung. Die Frage nach der Wahl des Quellterms und insbesondere nach der Wahl des zeitlichen Verlaufs der Quellintensität für das Ausbreitungsmodell wird hinsichtlich praktischer Anwendungen untersucht. Für verschiedene Randbedingungen werden analytische Lösungen der Ausbreitungsgleichung sowohl für den Fall reiner Diffusion als auch für den Fall von Diffusion-Advektion auf der Basis der Greenschen Funktion hergeleitet. Die wichtigsten Ergebnisse der theoretischen Ausbreitungsmodellierung werden in Hinblick auf die Quellenlokalisierung zusammengefasst.

Kapitel 3 beschreibt ein neues Verfahren zur Quellenlokalisierung für den Fall reiner Diffusion. Die Anwendung des geometrischen Zugangs führt auf ein zweistufiges Verfahren, das zunächst so genannte skalierbare Sensor-Quell-Abstände ermittelt und daraus den Quellort bestimmt. Die Vorteile des neuen Verfahrens gegenüber bestehenden einstufigen Verfahren werden herausgearbeitet. Es wird gezeigt, dass durch den neuen Ansatz 
Aussagen zur Existenz und Eindeutigkeit von Lösungen sowie zur Wahl geeigneter Sensorstandorte für die Quellenlokalisierung getroffen werden können.

Kapitel 4 befasst sich mit der Anwendung des geometrischen Zugangs auf den Fall von Diffusion-Advektion. Dafür wird die Idee der skalierbaren Sensor-Quell-Abstände auf so genannte verallgemeinerte skalierbare Sensor-Quell-Abstände erweitert.

Kapitel 5 zeigt die Leistungsfähigkeit der neuen Verfahren anhand zahlreicher Simulationsbeispiele. Schließlich wird die praktische Anwendbarkeit des neuen Verfahrens auf der Basis von Messungen mit räumlich verteilten Elektronischen Nasen in einem industriellen Chemikalienlager nachgewiesen.

Kapitel 6 fasst die Ergebnisse der Arbeit zusammen und gibt einen Ausblick auf zukünftige Arbeiten und weitere mögliche Anwendungsfelder der hier vorgestellten Verfahren. 


\section{Modellierung der Ausbreitung von Luftbeimengungen}

\subsection{Diffusions-Advektions-Gleichung}

Die Ausbreitung von Luftbeimengung [69, 97, 115] ist im Wesentlichen durch

- den diffusiven Transport (Diffusion) und

- den advektiven Transport (Advektion)

gekennzeichnet. Weitere Einflussfaktoren, wie die chemische Umwandlung der Luftbeimengung oder die Absorption der Luftbeimengung an Oberflächen, sind oft vernachlässigbar und werden hier nicht berücksichtigt.

Ursache der Diffusion sind die Brownsche Molekularbewegung (molekulare Diffusion) und die turbulente Bewegung der Luft (turbulente Diffusion/Eddy-Diffusion). Die molekulare Diffusion lässt sich durch die Diffusionsgleichung

$$
\frac{\partial C}{\partial t}=\frac{\partial}{\partial x}\left(K_{x} \frac{\partial C}{\partial x}\right)+\frac{\partial}{\partial y}\left(K_{y} \frac{\partial C}{\partial y}\right)+\frac{\partial}{\partial z}\left(K_{z} \frac{\partial C}{\partial z}\right)
$$

mit den Diffusionskoeffizienten $K_{x}, K_{y}, K_{z}$ beschreiben. Sind die Diffusionskoeffizienten richtungsabhängig $\left(K_{x} \neq K_{y} \neq K_{z}\right)$ handelt es sich um anisotrope Diffusion. Die molekularen Diffusionskoeffizienten sind sehr klein (z. B. $K=8.6 \cdot 10^{-6} \mathrm{~m}^{2} / \mathrm{s}$ [22] für Toluol in Luft). Einen wesentlich größeren Einfluss auf die Ausbreitung hat die turbulente Diffusion. Sie wird durch thermische Effekte, sich bewegende Objekte, Wind usw. hervorgerufen. Durch kleine, im Vergleich zur Gesamtausbreitung mikroskopische Verwirbelungen (Turbulenzen) findet dabei ein Stofftransport statt. Die turbulente Diffusion ist sehr komplex und somit mathematisch schwer modellierbar. Dennoch stellt die Diffusionsgleichung (2.1) eine gute Approximation für die turbulente Diffusion dar [83], insbesondere dann, wenn eine Mittelung der Konzentrationsmesswerte erfolgt. Der turbulente Diffusionskoeffizient ist wesentlich größer als der molekulare Diffusionskoeffizient. Sein Wert ist weitgehend 
unabhängig von der betrachteten Substanz (Luftbeimengung), aber stark abhängig von den oben genannten äußeren Einflüssen.

Sind die Diffusionskoeffizienten ortsunabhängig, wird von Fickscher Diffusion gesprochen

$$
\frac{\partial C}{\partial t}=K_{x} \frac{\partial^{2} C}{\partial x^{2}}+K_{y} \frac{\partial^{2} C}{\partial y^{2}}+K_{z} \frac{\partial^{2} C}{\partial z^{2}} .
$$

Für konstante Diffusionskoeffizienten $K_{x}, K_{y}, K_{z}$ stellt diese Gleichung eine lineare partielle Differenzialgleichung 2. Ordnung vom parabolischen Typ dar ${ }^{1}$.

In einem isotropen Medium sind die Diffusionskoeffizienten richtungsunabhängig $\left(K_{x}=\right.$ $\left.K_{y}=K_{z} \equiv K\right)$, und es gilt die isotrope Diffusionsgleichung

$$
\frac{\partial C}{\partial t}=K\left(\frac{\partial^{2} C}{\partial x^{2}}+\frac{\partial^{2} C}{\partial y^{2}}+\frac{\partial^{2} C}{\partial z^{2}}\right)
$$

Advektion ist die Ausbreitung der Luftbeimengung mit der mittleren Strömungsrichtung (Luftbewegungsrichtung). An jedem Punkt des Ausbreitungsgebiets lässt sich die zeitliche Änderung der Konzentration $C$ der Luftbeimengung bei einer Strömung mit dem Geschwindigkeitsvektor $\mathbf{v}=\left(v_{x}, v_{y}, v_{z}\right)^{T}$ durch die Advektionsgleichung (lineare partielle Differenzialgleichung 1. Ordnung)

$$
\frac{\partial C}{\partial t}=-\left(v_{x} \frac{\partial C}{\partial x}+v_{y} \frac{\partial C}{\partial y}+v_{z} \frac{\partial C}{\partial z}\right)
$$

beschreiben.

In dieser Arbeit wird die Ausbreitung durch Advektion und Ficksche Diffusion betrachtet. Dafür folgt mit 2.4 und 2.2 die Diffusions-Advektions-Gleichung (lineare partielle Differenzialgleichung 2. Ordnung vom parabolischen Typ)

$$
\frac{\partial C}{\partial t}=-\left(v_{x} \frac{\partial C}{\partial x}+v_{y} \frac{\partial C}{\partial y}+v_{z} \frac{\partial C}{\partial z}\right)+K_{x} \frac{\partial^{2} C}{\partial x^{2}}+K_{y} \frac{\partial^{2} C}{\partial y^{2}}+K_{z} \frac{\partial^{2} C}{\partial z^{2}}
$$

\footnotetext{
${ }^{1}$ Um den Typ einer linearen partiellen Differenzialgleichung 2. Ordnung mit konstanten Koeffizienten zu bestimmen, müssen die Koeffizienten betrachtet werden, die in der durch lineare homogene Transformation der unabhängigen Variablen erhaltenen Normalform vor Gliedern mit einer Ableitung zweiter Ordnung stehen. Parabolisch: ein Koeffizient verschwindet, die übrigen sind verschieden von null und besitzen gleiches Vorzeichen. (Bei der Diffusionsgleichung verschwindet der Koeffizient vor der zweiten Ableitung der Konzentration nach der Zeit.) Elliptisch: alle Koeffizienten sind verschieden von null und besitzen gleiches Vorzeichen. Hyperbolisch: alle Koeffizienten sind verschieden von null, ein Koeffizient besitzt ein anderes Vorzeichen.[14]
} 


\subsection{Vereinfachung der Ausbreitungsgleichung}

Wie bereits in [94] beschrieben, lässt sich die Diffusions-Advektions-Gleichung für den anisotropen Fall und beliebige Strömungsgeschwindigkeitskomponenten $v_{x}, v_{y}, v_{z}$

$$
\frac{\partial C}{\partial t}=-\left(v_{x} \frac{\partial C}{\partial x}+v_{y} \frac{\partial C}{\partial y}+v_{z} \frac{\partial C}{\partial z}\right)+K_{x} \frac{\partial^{2} C}{\partial x^{2}}+K_{y} \frac{\partial^{2} C}{\partial y^{2}}+K_{z} \frac{\partial^{2} C}{\partial z^{2}}
$$

durch Koordinatentransformation auf den isotropen Fall $\left(K_{x}=K_{y}=K_{z} \equiv K\right)$ mit Strömung in $x$-Richtung $\left(v_{y}=v_{z} \equiv 0\right)$

$$
\frac{\partial C}{\partial t}=-v_{x} \frac{\partial C}{\partial x}+K\left(\frac{\partial^{2} C}{\partial x^{2}}+\frac{\partial^{2} C}{\partial y^{2}}+\frac{\partial^{2} C}{\partial z^{2}}\right)
$$

überführen. Dies wird nachfolgend ausführlich gezeigt.

Zunächst müssen die richtungsabhängigen Diffusionskoeffizienten eliminiert werden. Dazu wird eine Koordinatentransformation durch die Substitutionen

$$
\tilde{t}:=t, \quad \tilde{x}:=x, \quad \tilde{y}:=a \cdot y, \quad \tilde{z}:=b \cdot z
$$

vorgenommen. Die Diffusions-Advektions-Gleichung in den neuen Variablen lautet

$$
\frac{\partial C}{\partial \tilde{t}}=-\left(v_{x} \frac{\partial C}{\partial \tilde{x}}+\left(v_{y} \cdot a\right) \frac{\partial C}{\partial \tilde{y}}+\left(v_{z} \cdot b\right) \frac{\partial C}{\partial \tilde{z}}\right)+K_{x} \frac{\partial^{2} C}{\partial \tilde{x}^{2}}+\left(K_{y} \cdot a^{2}\right) \frac{\partial^{2} C}{\partial \tilde{y}^{2}}+\left(K_{z} \cdot b^{2}\right) \frac{\partial^{2} C}{\partial \tilde{z}^{2}}
$$

Durch Wahl von

$$
a=\sqrt{\frac{K_{x}}{K_{y}}} \text { und } b=\sqrt{\frac{K_{x}}{K_{z}}}
$$

ergibt sich dann die isotrope Diffusions-Advektions-Gleichung

$$
\frac{\partial C}{\partial \tilde{t}}=-\left(v_{\tilde{x}} \frac{\partial C}{\partial \tilde{x}}+v_{\tilde{y}} \frac{\partial C}{\partial \tilde{y}}+v_{\tilde{z}} \frac{\partial C}{\partial \tilde{z}}\right)+K_{\tilde{x}}\left(\frac{\partial^{2} C}{\partial \tilde{x}^{2}}+\frac{\partial^{2} C}{\partial \tilde{y}^{2}}+\frac{\partial^{2} C}{\partial \tilde{z}^{2}}\right)
$$

mit

$$
K_{\tilde{x}}=K_{x}, \quad v_{\tilde{x}}=v_{x}, \quad v_{\tilde{y}}=v_{y} \cdot a, \quad v_{\tilde{z}}=v_{z} \cdot b
$$

Wird jetzt das Koordinatensystem so gedreht, dass die aus $v_{\tilde{x}}, v_{\tilde{y}}, v_{\tilde{z}}$ resultierende Strömungsgeschwindigkeit in Richtung der $\bar{x}$-Achse des neuen Koordinatensystems $(\bar{x}, \bar{y}, \bar{z})$ zeigt, dann folgt die gewünschte vereinfachte Diffusions-Advektions-Gleichung

$$
\frac{\partial C}{\partial \tilde{t}}=-v_{\bar{x}} \frac{\partial C}{\partial x}+K\left(\frac{\partial^{2} C}{\partial \bar{x}^{2}}+\frac{\partial^{2} C}{\partial \bar{y}^{2}}+\frac{\partial^{2} C}{\partial \bar{z}^{2}}\right)
$$

mit

$$
v_{\bar{x}}=\sqrt{v_{\tilde{x}}^{2}+v_{\tilde{y}}^{2}+v_{\tilde{z}}^{2}}, \quad v_{\bar{y}}=v_{\bar{z}} \equiv 0, \quad K=K_{\tilde{x}} .
$$

Die weiteren Untersuchungen können damit auf (2.7) beschränkt werden. 


\subsection{Wahl des Quellterms}

Gleichung (2.7) ist die homogene Diffusions-Advektions-Gleichung. Um das Verhalten der Konzentration $C(\mathbf{x}, t)$ bezüglich Zeit und Ort nach Eintreten einer Quelle beschreiben zu können, muss die inhomogene Gleichung

$$
\frac{\partial C}{\partial t}+v_{x} \frac{\partial C}{\partial x}-K\left(\frac{\partial^{2} C}{\partial x^{2}}+\frac{\partial^{2} C}{\partial y^{2}}+\frac{\partial^{2} C}{\partial z^{2}}\right)=Q(\mathbf{x}, t)
$$

mit dem Quellterm $Q(\mathbf{x}, t)$ betrachtet werden.

Da in den meisten Anwendungsfällen die Ausdehnung der Quelle in Relation zum Ausbreitungsgebiet klein ist, kann sie idealisiert als Punktquelle am Ort $\mathbf{x}_{0}=\left(x_{0}, y_{0}, z_{0}\right)^{T}$ modelliert werden. Für das hier untersuchte Leckageproblem ist es zudem typisch, dass jeweils nur eine Quelle aktiv ist. Der Fall mehrerer gleichzeitig aktiver Quellen entspricht eher einem Havariefall, der hier nicht betrachtet wird.

Wird die Quellintensität allgemein mit $q(t)$ gekennzeichnet, dann lautet der Quellterm

$$
Q(\mathbf{x}, t)=q(t) \cdot \delta\left(x-x_{0}\right) \cdot \delta\left(y-y_{0}\right) \cdot \delta\left(z-z_{0}\right)
$$

Dabei beschreibt $\delta(\cdot)$ die Dirac-Funktion ${ }^{2}$, die sich in (2.16) auf die Ortskoordinaten bezieht. Der Term $\delta\left(x-x_{0}\right) \cdot \delta\left(y-y_{0}\right) \cdot \delta\left(z-z_{0}\right)$ drückt aus, dass eine punktförmige Quelle am Ort $\left(x_{0}, y_{0}, z_{0}\right)$ wirkt.

Hinsichtlich des zeitlichen Verlaufs der Quellintensität $q(t)$ besitzen folgende Quellterme besondere praktische Relevanz:

- Die instantane (impulsförmige) Quellintensität $q(t)=M_{0} \cdot \delta\left(t-t_{0}\right)$ beschreibt idealisiert die plötzliche Freigabe der Menge $M_{0}$ des betrachteten Stoffs zum Zeitpunkt $t_{0}$. Ein solcher Verlauf der Intensität tritt beispielsweise beim Bersten/Platzen von kleinen Druckbehältern auf.

- Die sprungförmige Quellintensität $q(t)=q_{0} \cdot 1\left(t-t_{0}\right)$ beschreibt idealisiert, dass ab dem Zeitpunkt $t_{0}$ eine konstante Menge des betrachteten Stoffs pro Zeiteinheit $q_{0}$ austritt. Ein solcher Quellverlauf ist beispielsweise bei Leckagen von Rohrleitungen oder bei kleineren Leckagen großer Behälter zu beobachten.

Abb. 2.1 zeigt die zeitlichen Verläufe der Quellintensität $q(t)$ für beide Quellarten. Bei der instantanen Quelle entspricht die Fläche des Impulses der freigesetzten Menge $M_{0}$.

\footnotetext{
${ }^{2}$ Das Gleichheitszeichen in (2.16) ist wegen der Dirac-Funktionen auf der rechten Seite nur im Sinne der Distributionentheorie [33, 101] zu verstehen (vgl. auch Anhang B). Ohne weiteren Kommentar werden Gleichheitszeichen in Gleichungen, die $\delta$-Funktionen enthalten, in diesem Sinne aufgefasst.
} 

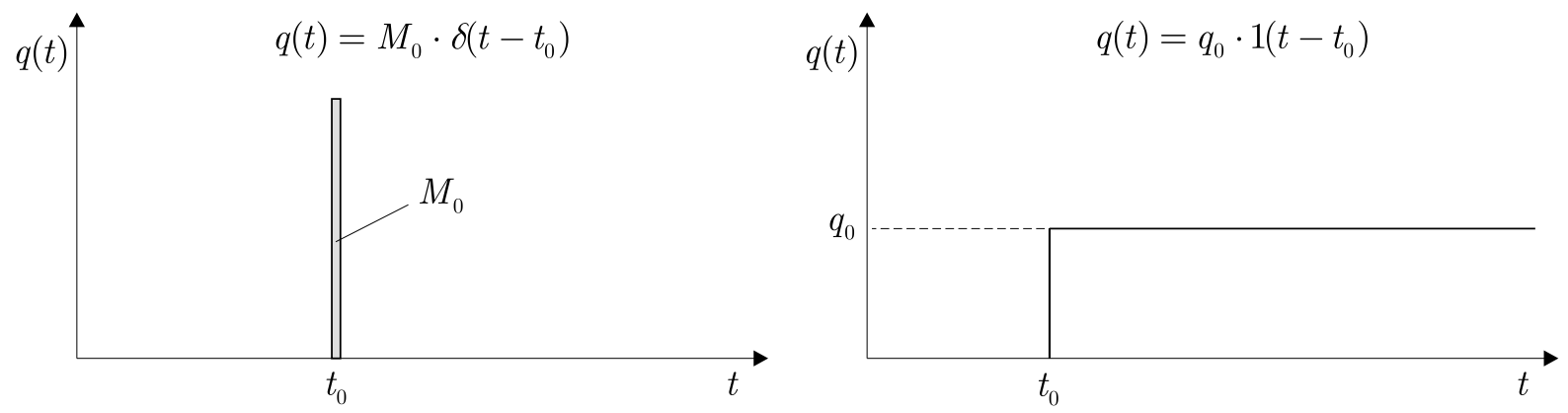

Abb. 2.1: Zeitlicher Verlauf der Quellintensität für instantane und sprungförmige Quelle.

Nachfolgend werden für diese beiden Quellterme analytische Lösungen der DiffusionsAdvektions-Gleichung hergeleitet.

\subsection{Lösungen der Diffusions-Advektions-Gleichung}

\subsubsection{Greensche Funktion}

Wird die Ausbreitung einer Luftbeimengung im betrachteten Gebiet nicht durch Hindernisse (z. B. Wände) beeinflusst, so kann idealisiert ein unendliches dreidimensionales Ausbreitungsmedium für die Modellierung zugrunde gelegt werden. Diese Idealisierung erlaubt analytische Lösungen für die Diffusionsgleichung.

Für das Finden solcher Lösungen bietet sich die Greensche Funktion an [9]. Eine Greensche Funktion beschreibt die Konzentration $C$ am Ort $\mathbf{x}=(x, y, z)^{T}$ zur Zeit $t$ infolge einer instantanen, punktförmigen Quelle im Punkt $\mathbf{x}_{0}=\left(x_{0}, y_{0}, z_{0}\right)^{T}$, zum Zeitpunkt $t_{0}$, mit der Stärke 1 , wenn vor $t_{0}$ die Konzentration $C$ überall gleich null war $[18,50,116] .{ }^{3}$

Für den Fall reiner Diffusion muss die gesuchte Greensche Funktion $C_{G}(\mathbf{x}, t)$ also für $t \geq t_{0}$ und beliebiges $\mathbf{x}$ die Gleichung

$$
\frac{\partial C_{G}}{\partial t}-K\left(\frac{\partial^{2} C_{G}}{\partial x^{2}}+\frac{\partial^{2} C_{G}}{\partial y^{2}}+\frac{\partial^{2} C_{G}}{\partial z^{2}}\right)=\delta\left(t-t_{0}\right) \cdot \delta\left(x-x_{0}\right) \cdot \delta\left(y-y_{0}\right) \cdot \delta\left(z-z_{0}\right)
$$

für die Anfangsbedingungen $C(\mathbf{x}, t) \equiv 0, t<t_{0}$ erfüllen. Hierfür lautet die Greensche Funktion [18, 21] (Nachweis in Anhang B)

$$
C_{G}(\mathbf{x}, t)=\frac{1}{8\left(\pi K\left(t-t_{0}\right)\right)^{\frac{3}{2}}} \cdot \exp \left(-\frac{\left\|\mathbf{x}-\mathbf{x}_{0}\right\|_{2}^{2}}{4 K\left(t-t_{0}\right)}\right) \cdot 1\left(t-t_{0}\right) .
$$

\footnotetext{
${ }^{3}$ Die Greensche Funktion entspricht im Fall von gewöhnlichen Differenzialgleichungen in $t$ der Gewichtsfunktion.
} 
Gleichung (2.18) stellt eine Greensche Funktion für den Fall dreidimensionaler Ausbreitung dar. Die allgemeine Greensche Funktion für den $n$-dimensionalen Fall ${ }^{4}$ lautet

$$
C_{G}(\mathbf{x}, t)=\left(\frac{1}{2 \sqrt{\pi K\left(t-t_{0}\right)}}\right)^{n} \cdot \exp \left(-\frac{\left\|\mathbf{x}-\mathbf{x}_{0}\right\|_{2}^{2}}{4 K\left(t-t_{0}\right)}\right) \cdot 1\left(t-t_{0}\right),
$$

wobei die Vektoren $\mathbf{x}, \mathbf{x}_{0}$ die Dimension $n$ besitzen. Insbesondere gilt für $t_{0}=0$

$$
C_{G, 0}(\mathbf{x}, t)=\left(\frac{1}{2 \sqrt{\pi K t}}\right)^{n} \cdot \exp \left(-\frac{\left\|\mathbf{x}-\mathbf{x}_{0}\right\|_{2}^{2}}{4 K t}\right) \cdot 1(t)
$$

Auf der Basis von (2.19) lässt sich die Greensche Funktion für den Fall von Advektion herleiten. Dabei wird ausgenutzt, dass (2.19) auch bei Advektion gilt, wenn sich das Koordinatensystem mit der Strömungsgeschwindigkeit $v_{x}$ in Richtung der $x$-Achse bewegt. Wird also die $x$-Koordinate durch

$$
x \rightarrow x-v_{x} \cdot\left(t-t_{0}\right)
$$

ersetzt, folgt als Greensche Funktion für den advektionsbehafteten Fall

$$
\begin{aligned}
C_{G, A}(\mathbf{x}, t) & =\left(\frac{1}{2 \sqrt{\pi K\left(t-t_{0}\right)}}\right)^{n} \\
& \times \exp \left(-\frac{\left(x-v_{x} \cdot\left(t-t_{0}\right)-x_{0}\right)^{2}+\left(y-y_{0}\right)^{2}+\left(z-z_{0}\right)^{2}}{4 K\left(t-t_{0}\right)}\right) \cdot 1\left(t-t_{0}\right)
\end{aligned}
$$

und für $t_{0}=0$

$$
\begin{aligned}
C_{G, A, 0}(\mathbf{x}, t) & =\left(\frac{1}{2 \sqrt{\pi K t}}\right)^{n} \\
& \times \exp \left(-\frac{\left(x-v_{x} \cdot t-x_{0}\right)^{2}+\left(y-y_{0}\right)^{2}+\left(z-z_{0}\right)^{2}}{4 K t}\right) \cdot 1(t) .
\end{aligned}
$$

\subsubsection{Diffusion im unendlichen Medium}

Durch Skalieren der Greenschen Funktion für dreidimensionale Medien $n=3$ mit $M_{0}$ wird die Lösung der Diffusionsgleichung für die instantane Quelle $C_{I}(\mathbf{x}, t)$ mit der Stärke $M_{0}$ erhalten

$$
\begin{aligned}
C_{I}(\mathbf{x}, t) & =M_{0} \cdot C_{G}(\mathbf{x}, t) \\
& =\frac{M_{0}}{8\left(\pi K\left(t-t_{0}\right)\right)^{\frac{3}{2}}} \cdot \exp \left(-\frac{\left\|\mathbf{x}-\mathbf{x}_{0}\right\|_{2}^{2}}{4 K\left(t-t_{0}\right)}\right) \cdot 1\left(t-t_{0}\right) .
\end{aligned}
$$

\footnotetext{
${ }^{4}$ Den Übergang der Greenschen Funktion vom dreidimensionalen zum zweidimensionalen Medium zeigt Anhang C.
} 
Die stationäre Lösung $(t \rightarrow \infty)$ für die instantane Quelle lautet

$$
C_{I}(\mathbf{x}, \infty)=0
$$

Die Lösung der Ausbreitungsgleichung für eine zeitlich kontinuierliche Quelle mit einem beliebigen Verlauf der Quellstärke $q(t)$ lässt sich durch Integrieren über die Zeit (Faltung)

$$
C(\mathbf{x}, t)=\int_{-\infty}^{\infty} q\left(t^{\prime}\right) \cdot C_{G, 0}\left(\mathbf{x}, t-t^{\prime}\right) \mathrm{d} t^{\prime}
$$

bestimmen. Für die sprungförmige Quellstärke $q(t)=q_{0} \cdot 1\left(t-t_{0}\right)$ folgt mit $(2.20)$

$$
C_{S}(\mathbf{x}, t)=\frac{q_{0}}{8(\pi K)^{\frac{3}{2}}} \cdot \int_{-\infty}^{\infty} 1\left(t^{\prime}-t_{0}\right) \cdot \frac{1}{\left(t-t^{\prime}\right)^{\frac{3}{2}}} \cdot \exp \left(-\frac{\left\|\mathbf{x}-\mathbf{x}_{0}\right\|_{2}^{2}}{4 K\left(t-t^{\prime}\right)}\right) \cdot 1\left(t-t^{\prime}\right) \mathrm{d} t^{\prime}
$$

und weiter

$$
C_{S}(\mathbf{x}, t)= \begin{cases}\frac{q_{0}}{8(\pi K)^{\frac{3}{2}}} \cdot \int_{t_{0}}^{t} \frac{1}{\left(t-t^{\prime}\right)^{\frac{3}{2}}} \cdot \exp \left(-\frac{\left\|\mathbf{x}-\mathbf{x}_{0}\right\|_{2}^{2}}{4 K\left(t-t^{\prime}\right)}\right) \mathrm{d} t^{\prime} & \text { für } t \geq t_{0} \\ 0 & \text { für } t<t_{0},\end{cases}
$$

also

$$
C_{S}(\mathbf{x}, t)=\frac{q_{0}}{8(\pi K)^{\frac{3}{2}}} \cdot \int_{t_{0}}^{t} \frac{1}{\left(t-t^{\prime}\right)^{\frac{3}{2}}} \cdot \exp \left(-\frac{\left\|\mathbf{x}-\mathbf{x}_{0}\right\|_{2}^{2}}{4 K\left(t-t^{\prime}\right)}\right) \mathrm{d} t^{\prime} \cdot 1\left(t-t_{0}\right)
$$

Als Greensche Funktion wurde bewusst (2.20) benutzt, da die Zeitverschiebung $t_{0}$ bereits durch die Quellintensität $q_{0} \cdot 1\left(t-t_{0}\right)$ berücksichtigt wird. Die Substitution von

$$
\tau=\frac{\left\|\mathbf{x}-\mathbf{x}_{0}\right\|_{2}}{2 \sqrt{K\left(t-t^{\prime}\right)}} \quad \text { mit } \quad \frac{d t^{\prime}}{d \tau}=\frac{4\left(K\left(t-t^{\prime}\right)\right)^{\frac{3}{2}}}{\left(\left\|\mathbf{x}-\mathbf{x}_{0}\right\|_{2} \cdot K\right)}
$$

führt auf

$$
C_{S}(\mathbf{x}, t)=\frac{q_{0}}{4 \pi K\left\|\mathbf{x}-\mathbf{x}_{0}\right\|_{2}} \cdot \frac{2}{\sqrt{\pi}} \int_{\frac{\left\|\mathbf{x}-\mathbf{x}_{0}\right\|_{2}}{2 \sqrt{K\left(t-t_{0}\right)}}}^{\infty} \exp \left(-\tau^{2}\right) \mathrm{d} \tau \cdot 1\left(t-t_{0}\right)
$$

bzw.

$$
C_{S}(\mathbf{x}, t)=\frac{q_{0}}{4 \pi K\left\|\mathbf{x}-\mathbf{x}_{0}\right\|_{2}} \cdot \operatorname{erfc}\left(\frac{\left\|\mathbf{x}-\mathbf{x}_{0}\right\|_{2}}{2 \sqrt{K\left(t-t_{0}\right)}}\right) \cdot 1\left(t-t_{0}\right) .
$$

Dabei bezeichnet $\operatorname{erfc}(\cdot)$ die komplementäre Fehler-Funktion ${ }^{5}[14,21,18]$.

${ }^{5}$ Es gilt $\operatorname{erfc}(x)=\frac{2}{\sqrt{\pi}} \int_{x}^{\infty} \exp \left(-\tau^{2}\right) \mathrm{d} \tau$. Der Zusammenhang zur Fehler-Funktion erf $(\cdot)$ lautet $\operatorname{erfc}(x)=1-\operatorname{erf}(x)$. Die Fehler-Funktion und das Gaußschen Fehlerintegral $\Phi_{0}(x)=\frac{1}{\sqrt{2 \pi}} \int_{0}^{x} \exp \left(\frac{-t^{2}}{2}\right) \mathrm{d} t$ stehen in enger Verbindung: $\operatorname{erf}(x)=2 \Phi_{0}(x \sqrt{2})$. Weiterhin ist $\lim _{x \rightarrow \infty} \operatorname{erf}(x)=1$. 
Den zeitlichen Verlauf der Konzentrationen an den in Abbildung 2.2 (links) dargestellten Sensoren gemäß Gl. (2.31) zeigt Abbildung 2.2 (rechts).
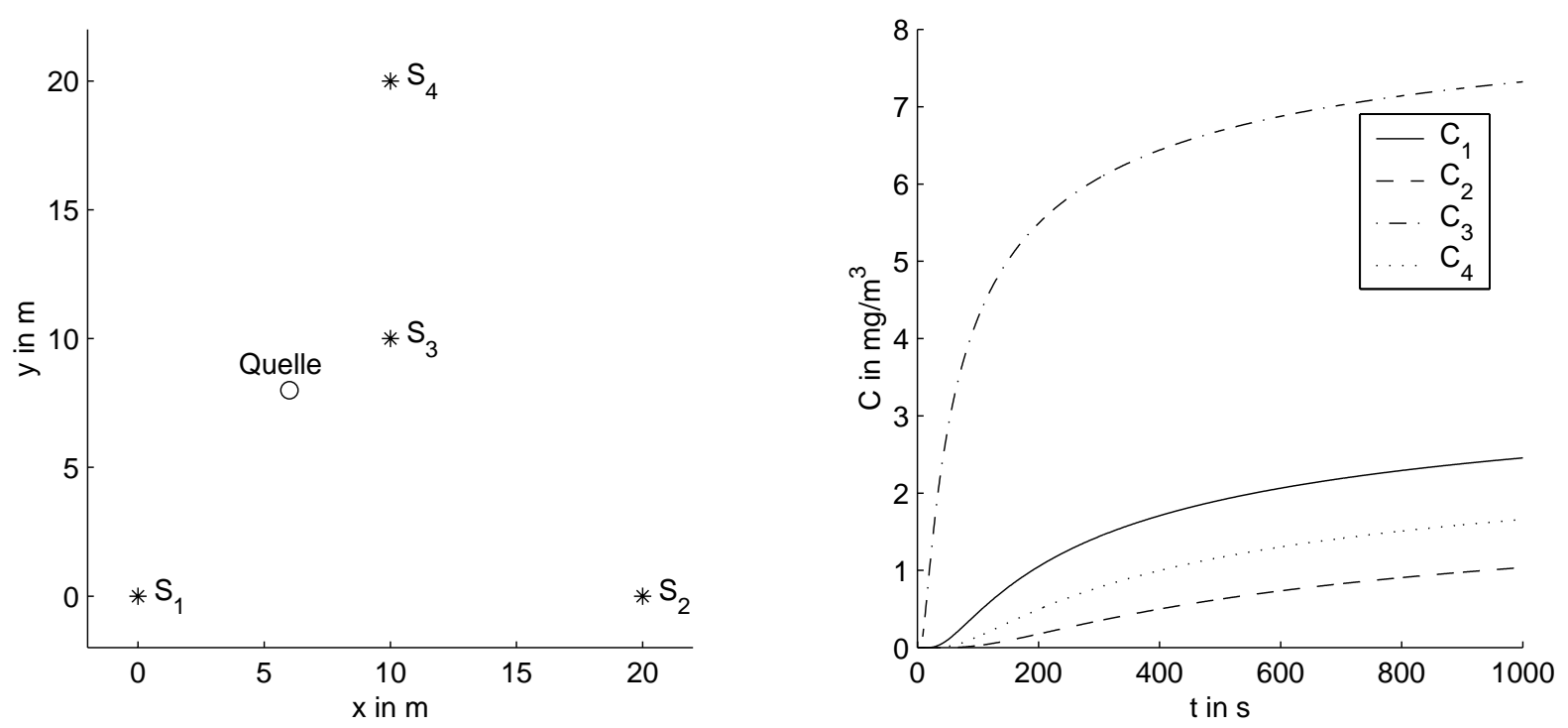

Abb. 2.2: Positionen der Sensoren und der Quelle $\mathbf{x}_{0}$ sowie zugehörige Konzentrationsverläufe bei Diffusion $\left(K=0.2 \mathrm{~m}^{2} / \mathrm{s}\right)$ mit sprungförmiger Quellintensität $\left(q_{0}=100 \mathrm{mg} / \mathrm{s}\right)$.

Wegen $\operatorname{erfc}(0)=1$ erreicht die Konzentration nach großer Zeit $t$ das stationäre Profil

$$
C_{S}(\mathbf{x}, \infty)=\frac{q_{0}}{4 \pi K\left\|\mathbf{x}-\mathbf{x}_{0}\right\|_{2}}
$$

Abbildung 2.3 zeigt das stationäre Konzentrationsprofil bei Diffusion über der $(x, y)$ Ebene.

Nach (2.31) strebt die Konzentration $C_{S}(\mathbf{x}, t)$ für $\left\|\mathbf{x}-\mathbf{x}_{0}\right\|_{2} \rightarrow 0$ gegen Unendlich. Diese Entartung resultiert aus der Annahme, dass ein endlicher Stofffluss $q_{0}$ durch einen Punkt, also durch ein unendlich kleines Volumen, in das System eingebracht wird. Praktisch wird am Quellort eine sehr hohe, endliche Konzentration vorliegen.

Abbildung 2.4 lässt erkennen, dass die Konvergenz der komplementären Fehler-Funktion relativ langsam ist. Daher wird das stationäre Konzentrationsprofil erst nach großer Zeit erreicht. 


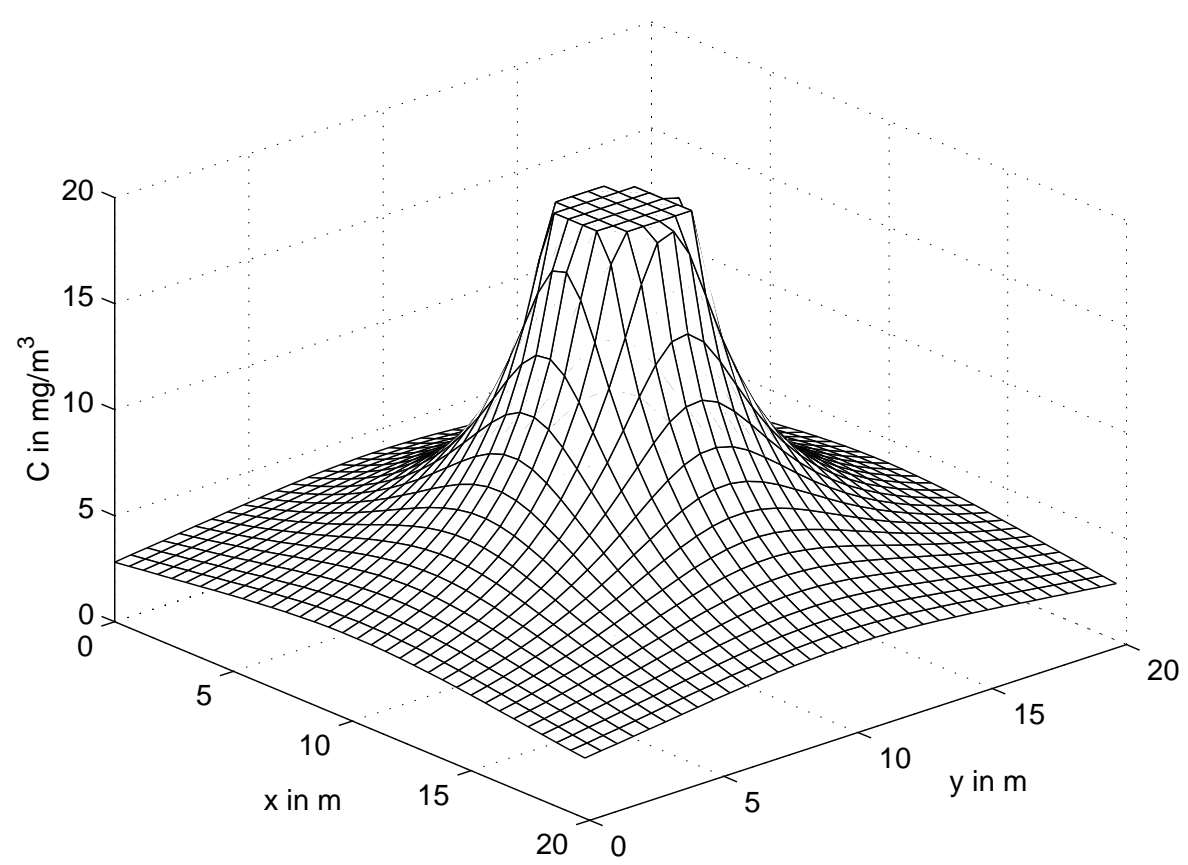

Abb. 2.3: Stationäres Konzentrationsprofil bei Diffusion $\left(K=0.2 \mathrm{~m}^{2} / \mathrm{s}\right)$ und sprungförmiger Quellintensität $q_{0}=100 \mathrm{mg} / \mathrm{s}$.

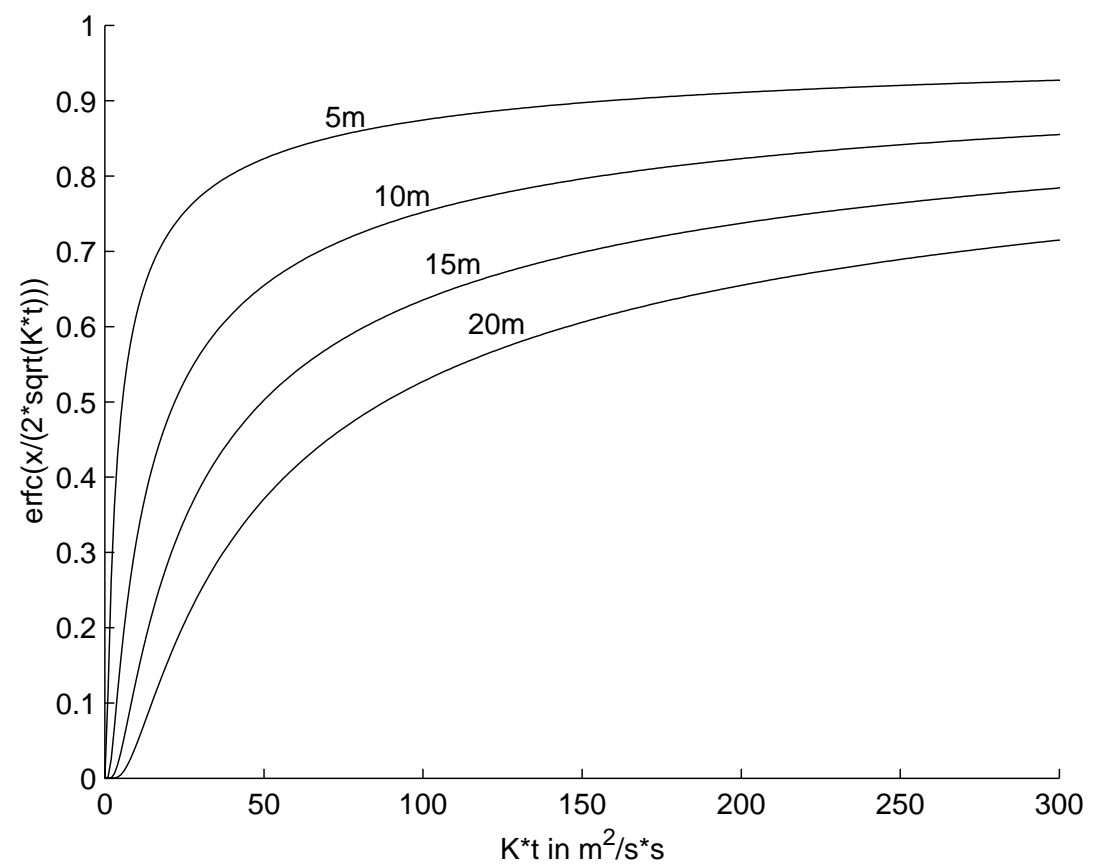

Abb. 2.4: Konvergenz des zeitabhängigen Terms gegen den Wert eins bei steigender Zeit für verschiedene Entfernungen zum Quellort. 


\subsubsection{Diffusion-Advektion im unendlichen Medium}

Die Lösung für den Fall dreidimensionaler Diffusion-Advektion lässt sich mit der Greenschen Funktion $C_{G, A}(2.22)$ herleiten. Für die instantane Quelle der Stärke $M_{0}$ folgt

$$
\begin{aligned}
C_{I, A}(\mathbf{x}, t)= & M_{0} \cdot C_{G, A}(\mathbf{x}, t) \\
= & \frac{M_{0}}{8\left(\pi K\left(t-t_{0}\right)\right)^{\frac{3}{2}}} \\
& \times \exp \left(-\frac{\left(x-v_{x} \cdot\left(t-t_{0}\right)-x_{0}\right)^{2}+\left(y-y_{0}\right)^{2}+\left(z-z_{0}\right)^{2}}{4 K\left(t-t_{0}\right)}\right) \cdot 1\left(t-t_{0}\right) .
\end{aligned}
$$

Die Lösung für die sprungförmige Quelle mit der Intensität $q_{0}$ wird unter Verwendung der Greenschen Funktion für $t_{0}=0$ beschrieben durch (vgl. (2.27) - (2.30))

$$
\begin{aligned}
C_{S, A}(\mathbf{x}, t) & =\frac{q_{0}}{8(\pi K)^{\frac{3}{2}}} \cdot \int_{-\infty}^{\infty} 1\left(t^{\prime}-t_{0}\right) \cdot \frac{1}{\left(t-t^{\prime}\right)^{\frac{3}{2}}} \\
& \times \exp \left(-\frac{\left(x-v_{x} \cdot\left(t-t^{\prime}\right)-x_{0}\right)^{2}+\left(y-y_{0}\right)^{2}+\left(z-z_{0}\right)^{2}}{4 K\left(t-t^{\prime}\right)}\right) \cdot 1\left(t-t^{\prime}\right) \mathrm{d} t^{\prime} \\
= & \frac{q_{0}}{8(\pi K)^{\frac{3}{2}}} \cdot \int_{t_{0}}^{t} \frac{1}{\left(t-t^{\prime}\right)^{\frac{3}{2}}} \\
\times & \exp \left(-\frac{\left(x-v_{x} \cdot\left(t-t^{\prime}\right)-x_{0}\right)^{2}+\left(y-y_{0}\right)^{2}+\left(z-z_{0}\right)^{2}}{4 K\left(t-t^{\prime}\right)} \mathrm{d} t^{\prime} \cdot 1\left(t-t_{0}\right)\right. \\
= & \frac{q_{0} \cdot \exp \left(\frac{v_{x}\left(x-x_{0}\right)}{2 K}\right)}{2 \pi^{\frac{3}{2}} K\left\|\mathbf{x}-\mathbf{x}_{0}\right\|_{2}} \int_{\frac{\left\|\mathbf{x}-\mathbf{x}_{0}\right\|_{2}}{2 \sqrt{K\left(t-t_{0}\right)}}}^{\infty} \exp \left(-\xi^{2}-\frac{v_{x}^{2} \cdot\left\|\mathbf{x}-\mathbf{x}_{0}\right\|_{2}^{2}}{16 K^{2} \xi^{2}}\right) d \xi \cdot 1\left(t-t_{0}\right)
\end{aligned}
$$

Für $t \rightarrow \infty$ wird ein stationäres Konzentrationsprofil

$$
C_{S}(\mathbf{x}, \infty)=\frac{q_{0} \cdot \exp \left(-\frac{v_{x}}{2 K}\left(\left\|\mathbf{x}-\mathbf{x}_{0}\right\|_{2}-\left(x-x_{0}\right)\right)\right)}{4 \pi K\left\|\mathbf{x}-\mathbf{x}_{0}\right\|_{2}}
$$

erreicht [18, 94]. Im Fall von Diffusion-Advektion existiert also eine geschlossene Lösung nur für die stationären, nicht aber für die instationären Konzentrationen.

In Abb. 2.5 sind die simulierten Konzentrationsverläufe für verschiedene Sensorpositionen dargestellt. Es ist zu erkennen, dass die stationären Werte schon nach kurzer Zeit erreicht 

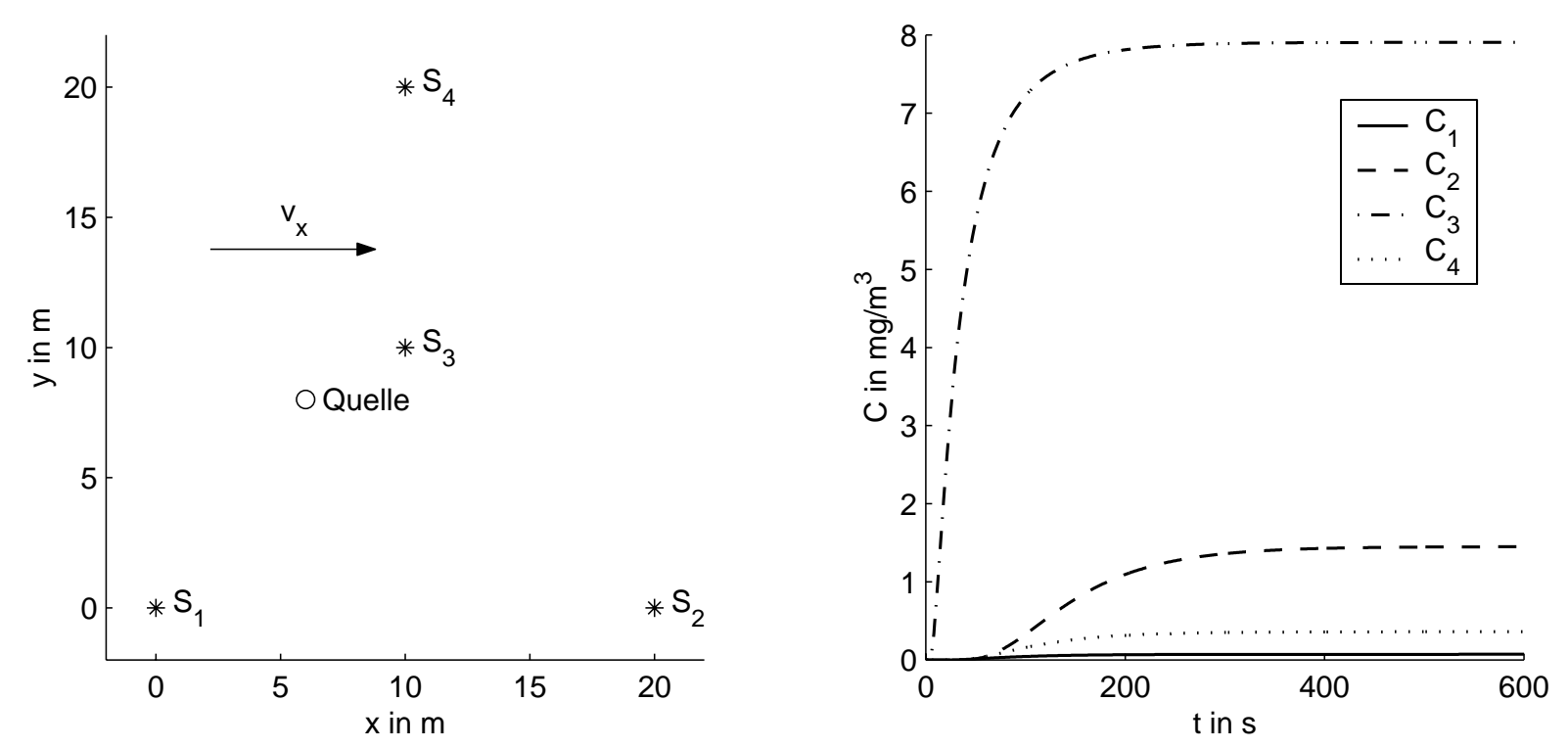

Abb. 2.5: Positionen der Sensoren und der Quelle sowie zugehörige Konzentrationsverläufe bei Diffusion-Advektion $\left(v_{x}=0.1 \mathrm{~m} / \mathrm{s}, K=0.2 \mathrm{~m}^{2} / \mathrm{s}\right)$ mit sprungförmiger Quellintensität $\left(q_{0}=\right.$ $100 \mathrm{mg} / \mathrm{s})$.

werden, obwohl hier nur eine kleine Strömungsgeschwindigkeit $v_{x}=0.1 \mathrm{~m} / \mathrm{s}$ angenommen wurde. Aus diesem Grund beschränken sich die weiteren Untersuchungen für den advektionsbehafteten Fall auf die stationäre Lösung (2.35).

Abbildung 2.6 zeigt das stationäre Konzentrationsprofil über der $(x, y)$-Ebene für den Fall von Diffusion-Advektion. Im Unterschied zur reinen Diffusion (Abb. 2.3) besitzt das Konzentrationsprofil hier einen Rücken in $x$-Richtung.

\subsubsection{Lösungen für das halbunendliche Medium}

Neben dem unendlichen Medium spielt bei der Ausbreitungsmodellierung der idealisierte Fall eines halbunendlichen Mediums eine zentrale Rolle. Unter einem halbunendlichen Medium wird ein Ausbreitungsgebiet verstanden, das in einer Dimension durch eine impermeable (undurchlässige) Fläche (hier bei $z=0$ ) begrenzt ist. Praktisch stellt meist der Boden diese impermeable Fläche dar. In dieser Arbeit wird nur der Fall betrachtet, bei dem sich die Quelle auf dieser impermeablen Fläche befindet $\left(z_{0}=0\right)$ und das Ausbreitungsgebiet nur für $z \geq 0$ definiert ist. Unter diesen Annahmen lassen sich die Lösungen der Diffusions-Advektions-Gleichung einfach aus den Lösungen für das unendliche Medium ableiten.

Für die impermeable Fläche bei $z=0$ gilt

$$
\frac{\partial C}{\partial z}=0
$$




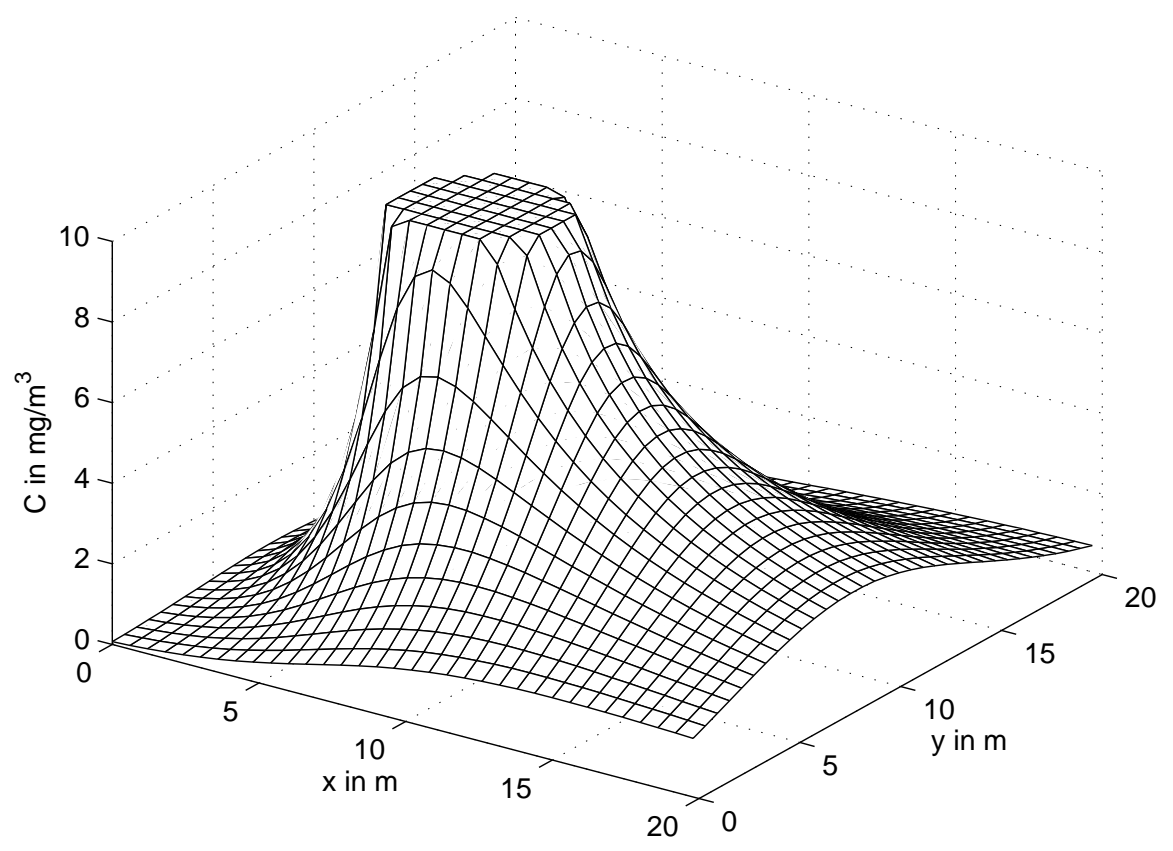

Abb. 2.6: Stationäres Konzentrationsprofil bei Diffusion-Advektion $\left(v_{x}=0.1 \mathrm{~m} / \mathrm{s}, K=0.2 \mathrm{~m}^{2} / \mathrm{s}\right)$ mit sprungförmiger Quellintensität $\left(q_{0}=100 \mathrm{mg} / \mathrm{s}\right)$.

Diese Bedingung ist dann erfüllt, wenn das Konzentrationsprofil als an der impermeablen Fläche gespiegelt und mit dem ursprünglichen Konzentrationsprofil superpositioniert angesehen wird [21].

Für den Fall $z_{0}=0$ findet diese angenommene Spiegelung direkt am Quellort statt. Das Konzentrationsprofil für $z<0$ für das unendliche Medium wird also mit dem Konzentrationsprofil für $z \geq 0$ superpositioniert. Da beide Profile identisch sind, führt das zu einer Verdopplung der Konzentrationen.

Damit lauten die Lösungen für den advektionsfreien Fall mit instantaner Quelle

$$
C_{I, H}(\mathbf{x}, t)=\frac{M_{0}}{4\left(\pi K\left(t-t_{0}\right)\right)^{\frac{3}{2}}} \cdot \exp \left(-\frac{\left\|\mathbf{x}-\mathbf{x}_{0}\right\|_{2}^{2}}{4 K\left(t-t_{0}\right)}\right) \cdot 1\left(t-t_{0}\right)
$$

und mit sprungförmiger Quelle

$$
C_{S, H}(\mathbf{x}, t)=\frac{q_{0}}{2 \pi K\left\|\mathbf{x}-\mathbf{x}_{0}\right\|_{2}} \cdot \operatorname{erfc}\left(\frac{\left\|\mathbf{x}-\mathbf{x}_{0}\right\|_{2}}{2 \sqrt{K\left(t-t_{0}\right)}}\right) \cdot 1\left(t-t_{0}\right),
$$

aus denen sich die stationären Lösungen

$$
\begin{gathered}
C_{I, H}(\mathbf{x}, \infty)=0 \quad \text { und } \\
C_{S, H}(\mathbf{x}, \infty)=\frac{q_{0}}{2 \pi K\left\|\mathbf{x}-\mathbf{x}_{0}\right\|_{2}}
\end{gathered}
$$

ablesen lassen. 
Für den advektionsbehafteten Fall ergeben sich für die instantane Quelle

$$
\begin{aligned}
C_{I, A, H}(\mathbf{x}, t)= & \frac{M_{0}}{4\left(\pi K\left(t-t_{0}\right)\right)^{\frac{3}{2}}} \\
& \times \exp \left(-\frac{\left(x-v_{x} \cdot\left(t-t_{0}\right)-x_{0}\right)^{2}+\left(y-y_{0}\right)^{2}+\left(z-z_{0}\right)^{2}}{4 K\left(t-t_{0}\right)}\right) \cdot 1\left(t-t_{0}\right)
\end{aligned}
$$

und für die sprungförmige Quelle

$$
C_{S, A, H}(\mathbf{x}, t)=\frac{q_{0} \cdot \exp \left(\frac{v_{x}\left(x-x_{0}\right)}{2 K}\right)}{\pi^{\frac{3}{2}} K\left\|\mathbf{x}-\mathbf{x}_{0}\right\|_{2}} \int_{\frac{\left\|\mathbf{x}-\mathbf{x}_{0}\right\|_{2}}{2 \sqrt{K\left(t-t_{0}\right)}}}^{\infty} \exp \left(-\xi^{2}-\frac{v_{x}^{2} \cdot\left\|\mathbf{x}-\mathbf{x}_{0}\right\|_{2}^{2}}{16 K^{2} \xi^{2}}\right) d \xi
$$

mit den stationären Lösungen

$$
C_{I, A, H}(\mathbf{x}, \infty)=0
$$

und

$$
C_{S, A, H}(\mathbf{x}, \infty)=\frac{q_{0} \cdot \exp \left(-\frac{v_{x}}{2 K}\left(\left\|\mathbf{x}-\mathbf{x}_{0}\right\|_{2}-\left(x-x_{0}\right)\right)\right)}{2 \pi K\left\|\mathbf{x}-\mathbf{x}_{0}\right\|_{2}}
$$

\subsubsection{Lösung und Diskussion für das endliche Medium}

Bei der Ausbreitungsmodellierung in endlichen Medien muss die Wechselwirkung mit den Mediengrenzen berücksichtigt werden. Hier werden impermeable Mediengrenzen betrachtet, die - wie schon beim halbunendlichen Medium erläutert - das Konzentrationsprofil, das sich ohne die Grenze außerhalb des betrachteten Gebiets ergibt, in das Medium hinein reflektieren. Dabei superpositionieren sich ursprüngliches und reflektiertes Konzentrationsprofil. Das bedeutet, es entsteht durch eine impermeable Grenze eine zusätzliche, virtuelle Quelle $\mathbf{x}_{0}^{\prime}$, deren Lage sich durch senkrechte Spiegelung der Position der tatsächlichen Quelle mit der impermeablen Grenze ergibt.

Im Fall des halbunendlichen Mediums musste nur eine Spiegelung betrachtet werden. Da sich dabei die Quelle direkt auf der impermeablen Oberfläche befand, brauchte die Lösung für das unendliche Medium nur mit dem Faktor 2 multipliziert werden.

Problematischer ist es, wenn, wie im Fall einer Halle mit den Ausmaßen $L_{x}, L_{y}, L_{z}$, mehrere impermeable Grenzen zu berücksichtigen sind. Wände, Boden und Decke der Halle stellen in erster Näherung impermeable Oberflächen dar. Damit entstehen neben der tatsächlichen Quelle $\mathbf{x}_{0}$ durch Reflexion weitere 6 virtuelle Quellen $\mathbf{x}_{0}^{\prime}$. Abbildung 2.7 zeigt die durch Reflexion an den Wänden entstehenden 4 virtuellen Quellen in der $(x, y)$-Ebene. 


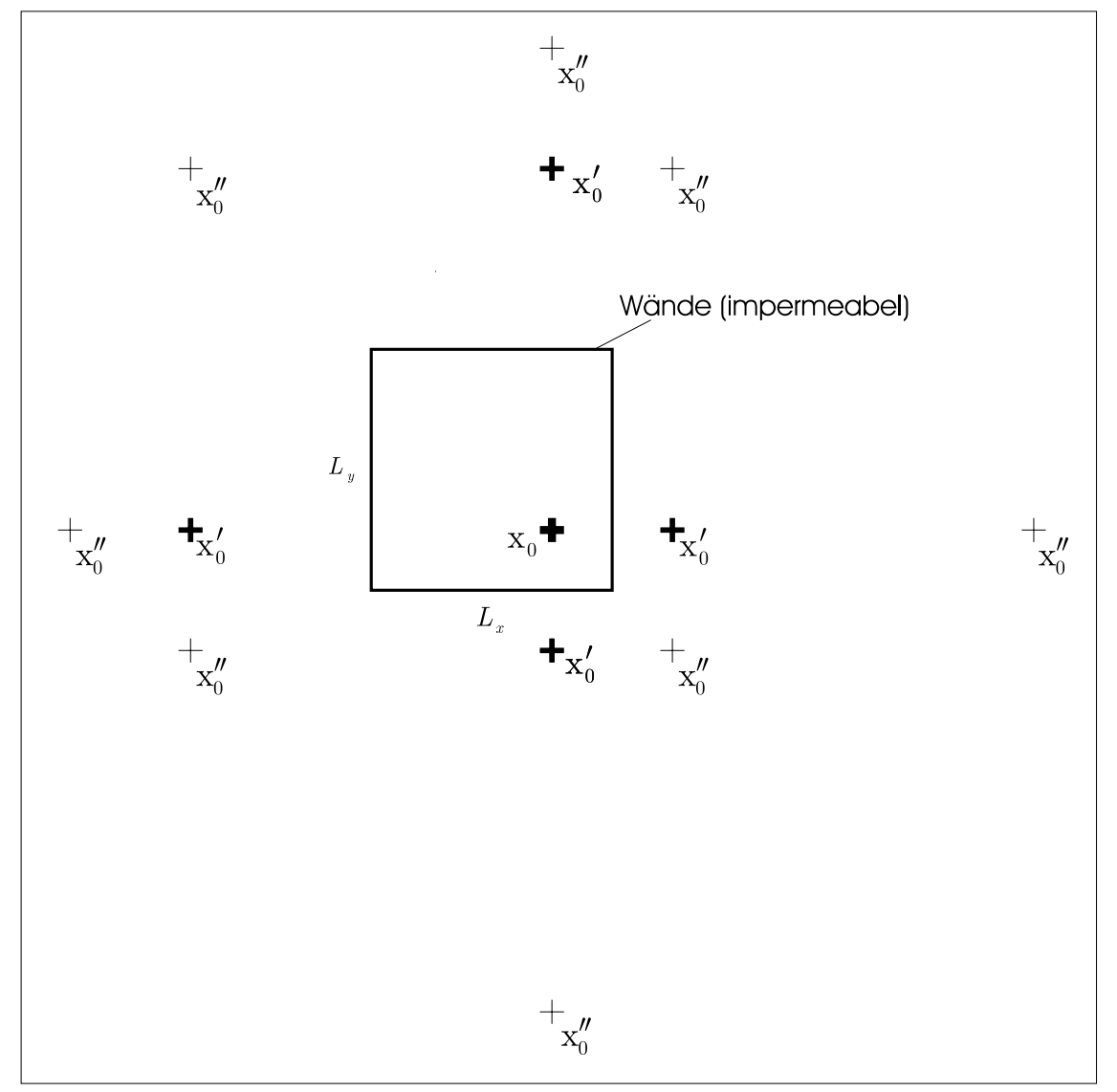

Abb. 2.7: Lage der durch Reflexion an den Wänden entstehenden virtuellen Quellen.

Doch auch die virtuellen Quellen müssen erneut an den impermeablen Oberflächen gespiegelt werden, wodurch die in Abb. 2.7 dargestellten virtuellen Quellen $\mathrm{x}_{0}^{\prime \prime}$ entstehen. Und auch die virtuellen Quellen $\mathrm{x}_{0}^{\prime \prime}$ müssen wieder reflektiert werden. Dieses Spiel lässt sich unendlich fortsetzen. Die Lösung für die Ausbreitungsgleichung ist damit eine unendliche Summe aus den durch die eigentliche Quelle und allen virtuellen Quellen erzeugten Konzentrationsprofilen. Für den Fall reiner Diffusion (advektionsfrei) lässt sich die Lösung mit (2.31) in der Form

$$
C_{S}(\mathbf{x}, t)=\sum_{i=-\infty}^{\infty} \sum_{j=-\infty}^{\infty} \sum_{k=-\infty}^{\infty} \frac{q_{0}}{4 \pi K\left\|\mathbf{x}-\mathbf{x}_{0}^{i, j, k}\right\|_{2}} \cdot \operatorname{erfc}\left(\frac{\left\|\mathbf{x}-\mathbf{x}_{0}^{i, j, k}\right\|_{2}}{2 \sqrt{K\left(t-t_{0}\right)}}\right) \cdot 1\left(t-t_{0}\right)
$$

schreiben [83]. Die $x$-Komponente der Quellorte $\mathbf{x}_{0}^{i, j, k}$ wird durch

$$
x_{0}^{i}=\left\{\begin{array}{rll}
x_{0} & : & i=0 \\
-x_{0}+i L_{x} & : & |i|=1 \\
\operatorname{sign}(i) x_{0}+i L_{x} & : & |i|>1
\end{array}\right.
$$

gebildet, was analog auch für die $y$ - und $z$-Komponente gilt. Dabei wurde ohne Einschränkung das Koordinatensystem so gewählt, dass der Mittelpunkt der Halle im Koordinatenursprung liegt. 
Mit zunehmender Ordnung der Spiegelung entfernen sich die virtuellen Quellen vom eigentlichen Ausbreitungsgebiet, so dass ihr Einfluss auf die Konzentrationsverteilung im betrachteten endlichen Medium sinkt. Dies gilt insbesondere für Zeitpunkte kurz nach Beginn der Emission. Deshalb kann die Summe in (2.45) eher abgebrochen werden. Laut [83] genügt für alle Zeitpunkte $t \leq t_{0}+\left[\min \left(L_{x}, L_{y}, L_{z}\right)\right]^{2} / 4 K$ die Summation mit $-1 \leq i, j, k \leq 1$. Doch auch mit dieser Beschränkung enthält die Lösung der Ausbreitungsgleichung bereits die tatsächliche und 26 weitere virtuelle Quellen (vgl. Gl. (2.45)). Für die hier angestrebte modellbasierte Quellenlokalisierung auf der Basis geschlossener Lösungen der Ausbreitungsgleichung ist diese Lösung ungeeignet.

Jedoch kann unter der Annahme, dass sich die Sensoren sowie die Quelle nicht unmittelbar in der Nähe einer Wand befinden, die Ausbreitung nach Beginn der Emission zunächst als freie Ausbreitung wie im unendlichen Medium aufgefasst werden. In der Zeit der freien Ausbreitung lässt sich dann die Quellenlokalisierung auf der Basis der Lösungen der Ausbreitungsgleichung für das unendliche Medium realisieren. Ab dem Zeitpunkt, an dem die Reflexionseinflüsse an den Wänden dominieren, muss das Ergebnis der Quellenlokalisierung eingefroren werden.

Für den Fall, dass sich die Quelle direkt in der Nähe einer Wand befindet, lässt sich die Quelle idealisiert so verschieben, dass sie sich direkt auf der Wand befindet. In diesem Fall gelten die Lösungen für das halbunendliche Medium, die sich von den Lösungen für das unendliche Medium nur durch den Faktor 2 unterscheiden. Befindet sich die Quelle in einer Kante muss statt des Faktors 2 der Faktor 4 verwendet werden. Ist die Quelle in einer Ecke positioniert, gilt die Lösung für das unendliche Medium multipliziert mit dem Faktor 8 .

Die Lokalisierung der Emissionsquelle ist ohne Kenntnis des konkret vorliegenden Faktors (1, 2, 4 oder 8) möglich, da verschiedene Faktoren als variable Quellintensität interpretiert werden können. Anhand des geschätzten Quellorts lässt sich der Faktor ermitteln und für die spätere Schätzung der Quellintensität verwenden.

\subsection{Lösungen für ein- und zweidimensionale Medien}

Neben der bislang betrachteten Ausbreitung in dreidimensionalen Medien existieren auch praktische Anwendungen, in denen eine Modellierung durch ein- oder zweidimensionale Medien sinnvoll ist. Beispielsweise lässt sich die Ausbreitung in Rohren, Gängen oder Kanälen durch die eindimensionale Diffusions-Advektions-Gleichung beschreiben. Dabei 
werden eine ideale Durchmischung in Querrichtung ${ }^{6}$ und impermeable Wände angenommen. Auch eine Modellierung durch zweidimensionale Medien kann bei sehr flachen Ausbreitungsgebieten sinnvoll sein.

Wegen der scheinbaren Ähnlichkeit der Greenschen Funktionen (2.19) und (2.22) für $n=$ 1,2,3 kann vermutet werden, dass die Lösungen der Ausbreitungsgleichung für ein- und zweidimensionale Ausbreitungsmedien den Lösungen für dreidimensionale Medien ähneln und sich direkt aus diesen ableiten lassen. Nachfolgend wird am Beispiel reiner Diffusion in Anlehnung an [18] gezeigt, dass diese Vermutung nicht zutrifft.

Im eindimensionalen Medium $(n=1)$ folgt mit (2.19) für reine Diffusion die Lösung bei instantaner Quelle der Stärke $M_{0}$

$$
C_{I}^{1 D}(x, t)=\frac{M_{0}}{2 \sqrt{\pi K\left(t-t_{0}\right)}} \cdot \exp \left(-\frac{\left|x-x_{0}\right|^{2}}{4 K\left(t-t_{0}\right)}\right) \cdot 1\left(t-t_{0}\right)
$$

Die Lösung für die sprungförmige Quelle wird analog zu (2.27)-(2.29) erhalten [19]

$$
C_{S}^{1 D}(x, t)=\frac{q_{0} \cdot \sqrt{t-t_{0}}}{K} \cdot \operatorname{ierfc}\left(\frac{\left|x-x_{0}\right|}{2 \sqrt{K\left(t-t_{0}\right)}}\right)
$$

Dabei bezeichnet ierfc $(\cdot)$ das Integral der komplementären Fehler-Funktion ${ }^{7}$. Für $t \rightarrow \infty$ existiert im eindimensionalen Medium keine stationäre Lösung, sondern es gilt

$$
C_{S}^{1 D}(x, \infty)=\infty
$$

Für das zweidimensionale Medium $(n=2)$ wird mit (2.19) für die instantane Quelle

$$
C_{I}^{2 D}(\mathbf{x}, t)=\left(\frac{M_{0}}{2 \sqrt{\pi K\left(t-t_{0}\right)}}\right)^{2} \cdot \exp \left(-\frac{\left\|\mathbf{x}-\mathbf{x}_{0}\right\|_{2}^{2}}{4 K\left(t-t_{0}\right)}\right) \cdot 1\left(t-t_{0}\right)
$$

erhalten. Analog zu (2.27)-(2.29) lässt sich die Lösung für die sprungförmige Quelle ermitteln

$$
C_{S}^{2 D}(\mathbf{x}, t)=-\frac{q_{0}}{4 \pi K} \cdot \operatorname{Ei}\left(-\frac{\left\|\mathbf{x}-\mathbf{x}_{0}\right\|_{2}^{2}}{4 K\left(t-t_{0}\right)}\right) .
$$

Dabei bezeichnet $\operatorname{Ei}(\cdot)$ die Integralexponentialfunktion ${ }^{8}[14,39]$. Wie auch im eindimensionalen Fall folgt für $t \rightarrow \infty$

$$
C_{S}^{2 D}(\mathbf{x}, \infty)=\infty
$$

\footnotetext{
${ }^{6}$ Die mit den nachfolgend gezeigten Lösungen der Diffusionsgleichung für ein- bzw. zweidimensionale Medien beschriebenen Konzentrationen besitzen die Einheit $\mathrm{mg} / \mathrm{m}^{2} \mathrm{bzw} . \mathrm{mg} / \mathrm{m}$. Diese müssen mit der Raumhöhe des idealisiert zweidimensionalen Mediums bzw. mit der Querschnittsfläche des idealisiert eindimensionalen Mediums (z. B. Rohrquerschnitt) dividiert werden, um echte Konzentrationswerte $\left(\mathrm{mg} / \mathrm{m}^{3}\right) \mathrm{zu}$ erhalten.

${ }^{7} \mathrm{Es}$ gilt $\operatorname{ierfc}(x)=\int_{x}^{\infty} \operatorname{erfc}(\mathrm{s}) \mathrm{ds}$.

${ }^{8} \operatorname{Es~gilt~} \operatorname{Ei}(x)=-\int_{-x}^{\infty} \frac{\exp (-\tau)}{\tau} d \tau=\int_{-\infty}^{x} \frac{\exp (\tau)}{\tau} d \tau \quad$ für $\quad x<0$. Die Forderung $x<0$ ist in $(2.51)$ stets erfüllt.
} 
Bereits die Lösungen (2.48) und (2.51) für reine Diffusion im ein- und zweidimensionalen unendlichen Medium lassen im Vergleich mit (2.31) erkennen, dass es sich bei der Ausbreitung im ein-, zwei- und dreidimensionalen Fall um jeweils strukturell völlig verschiedene Probleme handelt. Beispielsweise existieren im Gegensatz zum dreidimensionalen Fall keine stationären Lösungen für ein- und zweidimensionale Ausbreitungsmedien. Damit ergeben sich für die Quellenlokalisierung drei getrennt voneinander zu betrachtende Probleme. In dieser Arbeit wird nur der Fall der dreidimensionalen Ausbreitung untersucht. Untersuchungen zur Quellenlokalisierung für das eindimensionale Medium sind in [62] zu finden.

\subsection{Zusammenhang zwischen instantaner und sprungförmiger Quelle}

Bisher wurden die instantane und die sprungförmige Quelle getrennt betrachtet. Im Hinblick auf die Entwicklung von Verfahren zur Quellenlokalisierung ist es sinnvoll, nur die sprungförmige Quelle zu untersuchen und den instantanen Fall auf den sprungförmigen Fall zurückzuführen. Wie nachfolgend gezeigt wird, lässt sich dies wegen der Linearität der Diffusions-Advektions-Gleichung durch zeitliches Integrieren des Konzentrationsverlaufs für die instantane Quelle erreichen. Natürlich lässt sich auch der Fall sprungförmiger Quellintensität durch zeitliches Ableiten der Konzentrationen auf den instantanen Fall zurückführen. Doch ist aus der Sicht der Verarbeitung gestörter Messsignale ein Integrieren mit seiner glättenden Wirkung gegenüber dem Ableiten von Vorteil.

Der Nachweis wird hier anhand des advektionsfreien Falls über den Zusammenhang der Lösungen zur Greenschen Funktion $C_{G}(\mathbf{x}, t)$ geführt. Für die Konzentration $C_{I}(\mathbf{x}, t)$ bei instantaner Quelle gilt

$$
C_{I}(\mathbf{x}, t)=C_{G}(\mathbf{x}, t) \cdot M_{0} .
$$

In Anhang $\mathrm{D}$ ist für die Konzentration $C_{S}(\mathbf{x}, t)$ bei sprungförmigem Verlauf der Quellintensität die Beziehung

$$
\frac{\mathrm{d} C_{S}(\mathbf{x}, t)}{\mathrm{d} t}=C_{G}(\mathbf{x}, t) \cdot q_{0}
$$

nachgewiesen. Aus (2.53) und (2.54) folgt

$$
\frac{\mathrm{d} C_{S}(\mathbf{x}, t)}{\mathrm{d} t} \cdot \frac{1}{q_{0}}=C_{I}(\mathbf{x}, t) \cdot \frac{1}{M_{0}}
$$

und durch Integrieren über die Zeit wird

$$
C_{S}(\mathbf{x}, t) \cdot \frac{1}{q_{0}}=\int_{t_{0}}^{t} C_{I}\left(\mathbf{x}, t^{\prime}\right) \mathrm{d} t^{\prime} \cdot \frac{1}{M_{0}}
$$


erhalten. Damit ist gezeigt, dass der zeitlich integrierte Konzentrationsverlauf bei instantaner Quelle genauso behandelt werden kann wie der Konzentrationsverlauf bei sprungförmiger Quelle. Die Größen $q_{0}$ und $M_{0}$ sind dann betragsmäßig gleich. Dies gilt auch für den advektionsbehafteten Fall, wenn, wie hier vorausgesetzt, die Strömungsgeschwindigkeit für die Zeit der Ausbreitung konstant ist.

Bei Vorliegen einer instantanen Quelle müssen die im praktischen Einsatz zeitlich diskret gemessenen Konzentrationen über die Zeit summiert werden, um die nachfolgend gezeigten Verfahren zur Quellenlokalisierung verwenden zu können. Dabei ist zu beachten, dass die Summe zum Zeitpunkt $t_{0}$ den Wert 0 besitzen muss.

\subsection{Experimentelle Modellvalidierung}

Für die praktische Quellenlokalisierung ist, wegen des dabei zu lösenden inversen Problems, ein gutes Ausbreitungsmodell von entscheidender Bedeutung. Aus diesem Grund ist eine experimentelle Modellvalidierung bereits in einer frühen Phase sinnvoll. Bei der theoretischen Modellierung wurde angenommen, dass die Diffusions-Advektions-Gleichung mit den genannten idealisierenden Vereinfachungen das praktische Ausbreitungsverhalten hinreichend gut beschreibt.

Um dies zu überprüfen, wird ein bestehendes eindimensionales Sensornetz verwendet, das aus drei KAMINA (Karlsruher Mikronase) aufgebaut ist [62]. Das Sensornetz befindet sich im Flur des Instituts für Instrumentelle Analytik des Forschungszentrums Karlsruhe. Der Versuchsaufbau ist schematisch in Abb. 2.8 dargestellt.

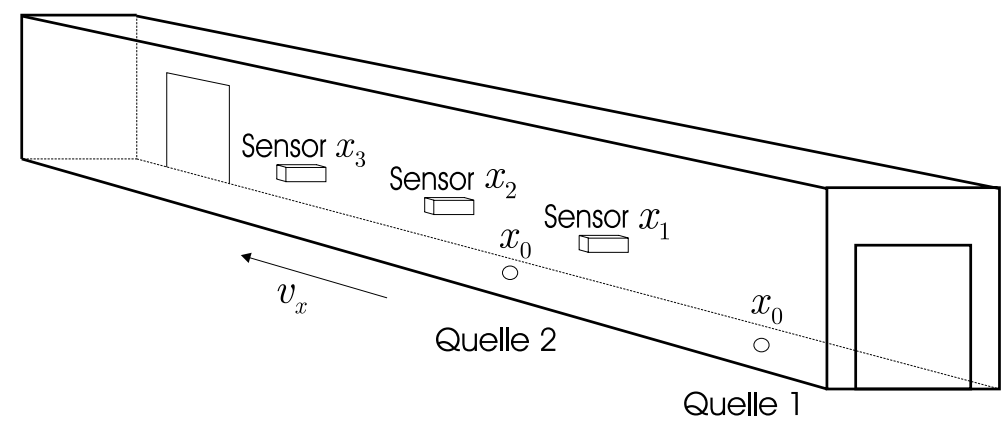

Abb. 2.8: Versuchsaufbau für die eindimensionale Ausbreitung durch Advektion und Diffusion.

Bei den Ausbreitungsversuchen wird an zwei verschiedenen Quellorten $x_{0}$ eine definierte Menge $M_{0}$ Ethanol freigesetzt. Dabei wird das flüssige Ethanol auf Filterpapier gekippt, von dem es aufgrund der großen Oberfläche sehr schnell verdunstet. Die Quellintensität kann damit idealisiert als instantan angesehen werden. 
Als Modell für die Ausbreitung wird die eindimensionale Diffusions-Advektions-Gleichung verwendet. Für die instantane Quelle folgt mit (2.22) die geschlossene Lösung

$$
C_{I, A}^{1 D}(x, t)=\frac{M_{0}}{2 \sqrt{\pi K\left(t-t_{0}\right)}} \cdot \exp \left(-\frac{\left(x-v_{x} \cdot\left(t-t_{0}\right)-x_{0}\right)^{2}}{4 K\left(t-t_{0}\right)}\right) \cdot 1\left(t-t_{0}\right) .
$$

Abbildung 2.9 zeigt sowohl die auf der Basis des Ausbreitungsmodells simulierten als auch die real gemessenen Konzentrationswerte an den drei Sensorstandorten $x_{1}, x_{2}$ und $x_{3}$ für zwei verschiedene Quellorte.
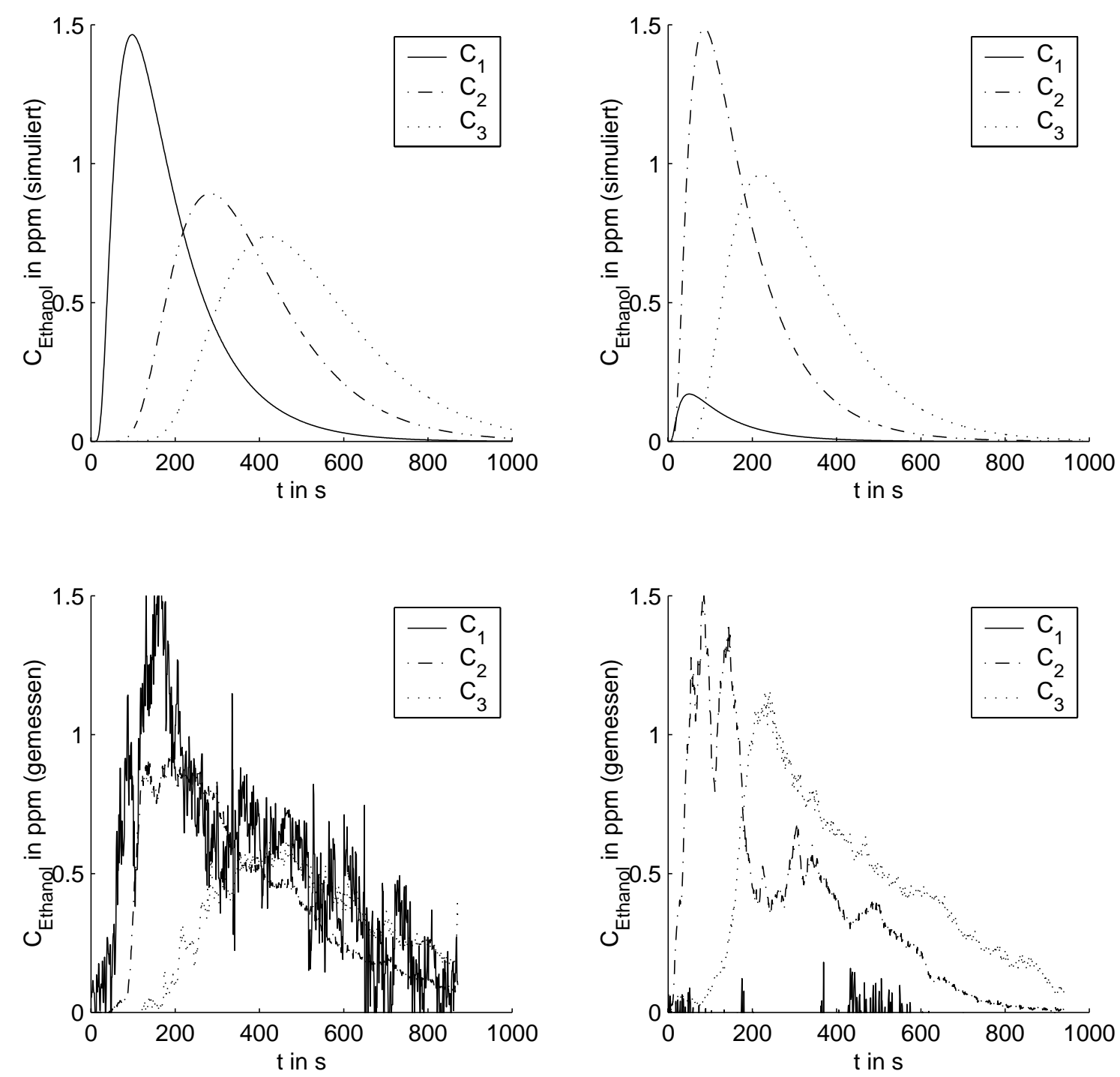

Abb. 2.9: Vergleich simulierter und gemessener Konzentrationsverläufe für den Flurversuch mit Ethanol $\left(K=0.2 \mathrm{~m}^{2} / \mathrm{s}, v_{x}=0.08 \mathrm{~m} / \mathrm{s}\right)$ für Quellort 1 (links) sowie Quellort 2 (rechts).

Die Abbildung zeigt, dass die Ausbreitung durch turbulente Diffusion und Advektion starke Schwankungen bei den gemessenen Konzentrationen hervorruft. Wie oben beschrieben, 
lässt sich die turbulente Ausbreitung nur in Näherung für zeitlich gemittelte Konzentrationen durch die Diffusions-Advektionsgleichung beschreiben. Kleine turbulente Wirbel können nicht explizit modelliert werden. Sie führen aber zu kurzzeitigem Ansteigen oder Absinken der Konzentrationen (vgl. z. B. Abb. 2.9 (rechts) bei ca. 150s). Zudem können kleine Konzentrationen im Messrauschen untergehen, wie im rechten Beispiel für Konzentration $C_{1}$ zu erkennen ist.

Unter Berücksichtigung der Schwierigkeiten bei der Modellierung der turbulenten Ausbreitung stellen die mit der Lösung der Advektions-Diffusionsgleichung ermittelten, simulierten Konzentrationsverläufe dennoch praktisch befriedigende Näherungen für die gemessenen Konzentrationen dar.

Jedoch kann es durch starke Störungen in den Strömungsverhältnissen im betrachteten Gebiet (Öffnen/Schließen von Türen/Fenstern, bewegte Objekte wie z. B. Personen) zu erheblichen Veränderungen des Ausbreitungsverhaltens kommen. In solchen Fällen können die simulativ ermittelten Konzentrationen wesentlich von den gemessenen abweichen. Das Ausbreitungsmodell ist dann nicht gültig. Da solche zufälligen Störungen aber nicht a priori bekannt sind, lässt sich i. Allg. kein besseres analytisches Ausbreitungsmodell finden.

\subsection{Konsequenzen für die Quellenlokalisierung}

Gegenstand dieses Kapitels war die theoretische Modellierung der Ausbreitung einer Luftbeimengung mittels der Diffusions-Advektions-Gleichung für verschiedene Randbedingungen. Anhand experimenteller Ergebnisse wurde gezeigt, dass die getroffenen Annahmen bezüglich der Ausbreitungsmodellierung praktisch zulässig sind.

Aus den theoretischen Untersuchungen ergeben sich folgende Konsequenzen:

- Die Ausbreitung im anisotropen dreidimensionalen Medium mit beliebigen Strömungsgeschwindigkeitskomponenten $v_{x}, v_{y}, v_{z}$ ist nach einer Koordinatentransformation durch ein Modell für das isotrope dreidimensionale Medium mit nur einer Strömungsgeschwindigkeitskomponente $v_{x}\left(v_{y}=v_{z}=0\right)$ beschreibbar. Die nachfolgenden Untersuchungen beschränken sich daher nur auf diesen Fall.

- Es werden ausschließlich punktförmige Quellen untersucht. Im Hinblick auf den zeitlichen Verlauf der Quellintensität sind praktisch die instantane und die sprungförmige Quelle relevant. Der Fall instantaner Quellintensität lässt sich durch Integrieren der gemessenen Konzentrationen über der Zeit auf den Fall sprungförmiger Quellintensität zurückführen, wobei betragsmäßig $M_{0}$ und $q_{0}$ übereinstimmen. Aus diesem 
Grund werden ausschließlich Lösungen des Quellenlokalisierungsproblems für den Fall einer sprungförmigen Quellintensität entwickelt. Für den instantanen Fall müssen dann die zeitlich summierten Signalverläufe betrachtet werden.

- Die Lösungen für das halbunendliche Medium mit einer Quelle auf der impermeablen Oberfläche unterscheiden sich nur durch den Faktor 2 von denen für das unendliche Medium. Aus diesem Grund wird im Weiteren vorrangig der Fall eines unendlichen Mediums untersucht. Wenn notwendig, wird auf Unterschiede der Verfahren zur Quellenlokalisierung für den Fall eines halbunendlichen Mediums hingewiesen.

- Für den Fall des abgeschlossenen (endlichen) Mediums sind die Lösungen komplizierter. Sie gestatten keine geschlossene Lösung für die Quellenlokalisierung und sind damit für die angestrebte theoretische Untersuchung ungeeignet. Allerdings kann die Ausbreitung nach Beginn der Emission wie eine freie Ausbreitung behandelt werden, vorausgesetzt die Quelle und das zu überwachende Gebiet sind weit genug von den impermeablen Grenzen entfernt. Weitere Spezialfälle ergeben sich, wenn sich die Quelle direkt an der Wand, in einer Kante oder einer Ecke des endlichen Mediums befindet.

- Sowohl beim advektionsfreien als auch beim advektionsbehafteten Fall erreichen die Konzentrationen im halbunendlichen und im unendlichen Medium nach einiger Zeit stationäre Endwerte. Das geschieht beim advektionsbehafteten Fall bereits bei geringen Strömungsgeschwindigkeiten schnell. Deshalb wird für diesen Fall nur die stationäre Lösung untersucht. Im advektionsfreien Fall ist die Konvergenz gegen die stationäre Lösung vergleichsweise langsam, so dass neben der stationären Lösung auch die instationäre Lösung betrachtet wird.

Damit müssen fortan nur noch die beiden Standardfälle

- reine Diffusion im dreidimensionalen unendlichen isotropen Medium mit sprungförmiger Punktquelle

$$
\frac{\partial C}{\partial t}-K\left(\frac{\partial^{2} C}{\partial x^{2}}+\frac{\partial^{2} C}{\partial y^{2}}+\frac{\partial^{2} C}{\partial z^{2}}\right)=q_{0} \cdot 1\left(t-t_{0}\right) \cdot \delta\left(x-x_{0}\right) \cdot \delta\left(y-y_{0}\right) \cdot \delta\left(z-z_{0}\right)
$$

mit der Lösung

$$
C(\mathbf{x}, t)=\frac{q_{0}}{4 \pi K\left\|\mathbf{x}-\mathbf{x}_{0}\right\|_{2}} \cdot \operatorname{erfc}\left(\frac{\left\|\mathbf{x}-\mathbf{x}_{0}\right\|_{2}}{2 \sqrt{K\left(t-t_{0}\right)}}\right) \cdot 1\left(t-t_{0}\right)
$$

und dem stationären Konzentrationsprofil

$$
C_{S}(\mathbf{x}, \infty)=\frac{q_{0}}{4 \pi K\left\|\mathbf{x}-\mathbf{x}_{0}\right\|_{2}}
$$

(Kapitel 3) sowie 
- Advektion in $x$-Richtung und Diffusion im dreidimensionalen unendlichen isotropen Medium mit sprungförmiger Punktquelle

$$
\begin{gathered}
\frac{\partial C}{\partial t}+v_{x} \frac{\partial C}{\partial x}-K\left(\frac{\partial^{2} C}{\partial x^{2}}+\frac{\partial^{2} C}{\partial y^{2}}+\frac{\partial^{2} C}{\partial z^{2}}\right)= \\
q_{0} \cdot 1\left(t-t_{0}\right) \cdot \delta\left(x-x_{0}\right) \cdot \delta\left(y-y_{0}\right) \cdot \delta\left(z-z_{0}\right)
\end{gathered}
$$

mit der Lösung

$$
C_{S}(\mathbf{x}, t)=\frac{q_{0} \cdot \exp \left(\frac{v_{x}\left(x-x_{0}\right)}{2 K}\right)}{2 \pi^{\frac{3}{2}} K\left\|\mathbf{x}-\mathbf{x}_{0}\right\|_{2}} \int_{\frac{\left\|\mathbf{x}-\mathbf{x}_{0}\right\|_{2}}{2 \sqrt{K\left(t-t_{0}\right)}}}^{\infty} \exp \left(-\xi^{2}-\frac{v_{x}^{2} \cdot\left\|\mathbf{x}-\mathbf{x}_{0}\right\|_{2}^{2}}{16 K^{2} \xi^{2}}\right) d \xi
$$

und dem stationären Konzentrationsprofil

$$
C_{S}(\mathbf{x}, \infty)=\frac{q_{0} \cdot \exp \left(-\frac{v_{x}}{2 K}\left(\left\|\mathbf{x}-\mathbf{x}_{0}\right\|_{2}-\left(x-x_{0}\right)\right)\right)}{4 \pi K\left\|\mathbf{x}-\mathbf{x}_{0}\right\|_{2}}
$$

(Kapitel 4) untersucht werden.

Zusammenfassend ergibt sich, dass ein großes Spektrum an Ausbreitungsproblemen auf diese beiden Standardfälle zurückgeführt werden kann. 


\section{Ein neues Verfahren zur Quellenlokalisierung bei Diffusion}

Wie im Kapitel 1 bereits beschrieben, besteht die Grundidee des hier vorgestellten geometrischen Zugangs zur Quellenlokalisierung darin, zunächst die Menge $\mathcal{P}_{i}$ zu bestimmen, auf der sich aus der Sicht jedes einzelnen Sensors $i$ die Quelle befinden kann. Durch Schneiden aller Mengen $\mathcal{P}_{i}$ soll daraus später der Quellort ermittelt werden.

Für den in diesem Kapitel betrachteten Fall isotroper Diffusion (ohne Advektion) beschreibt die Menge aller Punkte

$$
\mathcal{P}_{i}=\left\{\mathbf{x}_{0} \in \mathbb{R}^{3}:\left\|\mathbf{x}_{i}-\mathbf{x}_{0}\right\|_{2}=d_{i}\left(C_{i}, q_{0}, K\right)\right\}
$$

auf der sich aus der Sicht eines einzelnen Sensors $i$ die Quelle befinden kann, eine Kugel. Ihr Mittelpunkt ist der jeweilige Sensorstandort $\mathbf{x}_{i}$. Dies wird klar, wenn (3.1) als implizite Kugelgleichung

$$
\left(x_{i}-x_{0}\right)^{2}+\left(y_{i}-y_{0}\right)^{2}+\left(z_{i}-z_{0}\right)^{2}-d_{i}^{2}\left(C_{i}, q_{0}, K\right)=0
$$

geschrieben wird. Der Radius der Kugel, also der Sensor-Quell-Abstand $d_{i}\left(C_{i}, q_{0}, K\right)=$ $\left\|\mathbf{x}_{i}-\mathbf{x}_{0}\right\|_{2}$, ist jedoch neben der gemessenen Konzentration $C_{i}$ weiterhin von den i. Allg. unbekannten Größen Quellintensität $q_{0}$ und Diffusionskoeffizient $K$ abhängig.

Im Fall einer ebenen Anordnung von Sensoren und Quelle (Ebenenkonfiguration) stellt $\mathcal{P}_{i}$ für den Sensor $i$ einen Kreis um $\mathbf{x}_{i}$ dar. Für einen festen Diffusionskoeffizient $K$ ist der Radius dieses Kreises für eine vorliegende Konzentrationsmessung $C_{i}$ nur noch von der Quellintensität $q_{0}$ abhängig. Abbildung 3.1 verdeutlicht, dass sich für eine bestimmte Konzentrationsmessung $C_{i}$ die Quelle auf einem kleinen Kreis um $\mathbf{x}_{i}$ befinden kann, wenn eine kleine Quellintensität vorliegt, und umgekehrt auch auf einem großen Kreis um $\mathbf{x}_{i}$ liegen kann, wenn die Quellintensität groß ist. 


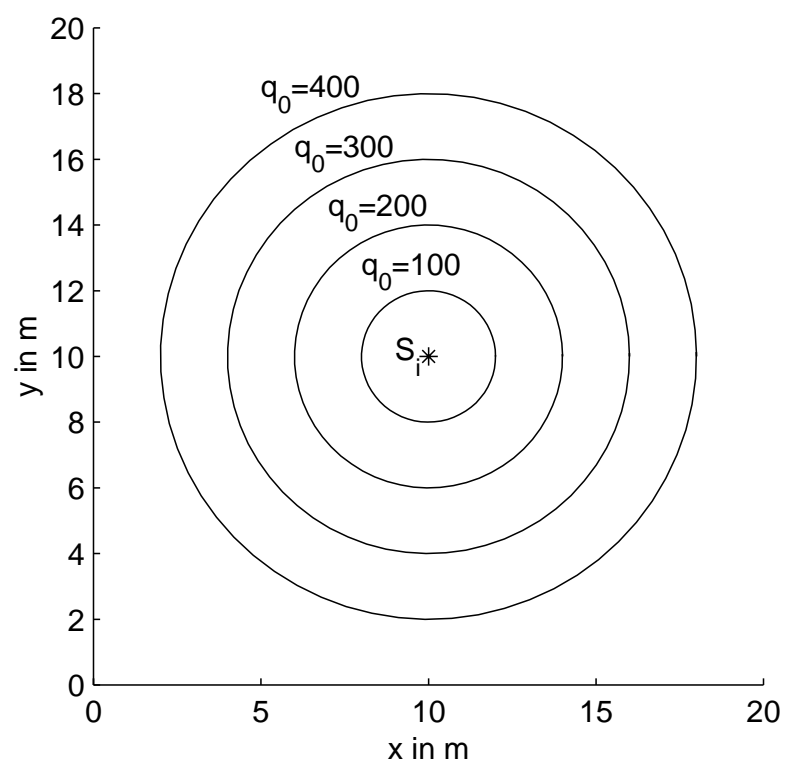

Abb. 3.1: Menge aller möglichen Quellorte $\mathcal{P}_{i}$ aus der Sicht von Sensor $i\left(S_{i}\right)$ für verschiedene Quellintensitäten $q_{0}(\mathrm{mg} / \mathrm{s})$ im advektionsfreien Fall.

Wie später gezeigt wird, bewirken die Parameter $K$ und $q_{0}$ eine lineare Skalierung der Sensor-Quell-Abstände $d_{i}\left(C_{i}, q_{0}, K\right)$. Aus diesem Grund lassen sich diese Abstände im Fall reiner Diffusion in

$$
d_{i}\left(C_{i}, q_{0}, K\right)=\frac{\varrho_{i}\left(C_{i}\right)}{\gamma\left(K, q_{0}\right)}
$$

zerlegen. Die Größe $\varrho_{i}\left(C_{i}\right)$ ist der so genannte skalierbare Sensor-Quell-Abstand. Der Skalierungsfaktor $\gamma$ hängt nur von den Parametern $q_{0}$ und $K$ ab. Daher besitzt $\gamma$ für alle Sensoren denselben Wert.

Erst durch die Betrachtung mehrerer Sensoren und damit mehrerer Mengen $\mathcal{P}_{i}$ lässt sich der Skalierungsfaktor sowie der Quellort bestimmen. Im ungestörten Fall ergibt sich bei Wahl des richtigen Skalierungsfaktors $\gamma$ (und damit $q_{0}$ ) ein Schnittpunkt aller $\mathcal{P}_{i}$ (Kreise/Kugeln), der sich genau am Quellort befindet (Abb. 3.2). Im gestörten Fall muss eine Näherungslösung für den Schnittpunkt ermittelt werden.

Beim zweistufigen Verfahren ist also zunächst für jeden Sensor $i$ ein skalierbarer SensorQuell-Abstand $\varrho_{i}$ zu schätzen (Abschnitt 3.1) und daraus anschließend der Quellort $\mathbf{x}_{0}$ zu bestimmen (Abschnitte 3.2 bis 3.4). 


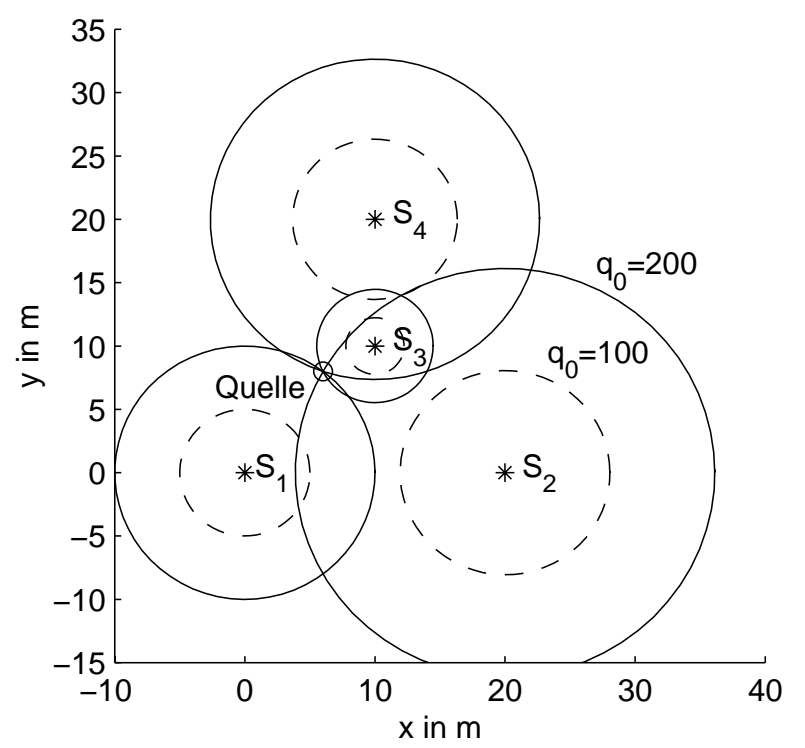

Abb. 3.2: Schnittpunkt aller $p$ Mengen $\mathcal{P}_{i}$ am Quellort $\mathbf{x}_{0}$ im ungestörten, advektionsfreien Fall. Wenn $q_{0}$ (und damit $\gamma$ ) falsch gewählt werden, existiert kein gemeinsamer Schnittpunkt der Kreise (gestrichelte Linie).

\subsection{Schätzung skalierbarer Sensor-Quell-Abstände}

\subsubsection{Schätzung auf der Basis stationärer Konzentrationen}

Zunächst wird die Schätzung skalierbarer Sensor-Quell-Abstände auf der Basis stationärer Konzentrationen (vgl. (2.60)) betrachtet

$$
C\left(\mathbf{x}_{i}, \infty\right)=\frac{q_{0}}{4 \pi K\left\|\mathbf{x}_{i}-\mathbf{x}_{0}\right\|_{2}} \quad i=1, \ldots, p
$$

Im gestörten Fall stellt der Mittelwert über mehrere nacheinander gemessene Werte $C_{i, k}$ ( $i$-ter Sensor zur Abtastzeit $t_{k}$ ) einen Schätzwert $\hat{C}\left(\mathbf{x}_{i}, \infty\right)$ dar, wenn $k$ eine große Zeit $t$ repräsentiert. Die Konzentrationswerte $C_{i, k}$ müssen dabei keiner äquidistanten Abtastung entstammen.

Werden (3.3) und (3.4) betrachtet, folgt für die skalierbaren Sensor-Quell-Abstände

$$
\varrho_{i}=\frac{1}{C\left(\mathbf{x}_{i}, \infty\right)} \quad\left(\text { ungestörter Fall) bzw. } \quad \hat{\varrho}_{i}=\frac{1}{\hat{C}\left(\mathbf{x}_{i}, \infty\right)} \quad\right. \text { (gestörter Fall) }
$$

mit dem zugehörigen Skalierungsfaktor

$$
\gamma=\frac{4 \pi K}{q_{0}} \quad \text { bzw. } \quad \gamma=\frac{2 \pi K}{q_{0}} \quad \text { (für das halbunendliche Medium). }
$$

Bei der Verwendung von $\varrho_{i}$ und $\gamma$ empfiehlt es sich, $\gamma$ als einheitenlose Größe zu betrachten und die physikalischen Einheiten von $K$ und $q_{0}$ den skalierbaren Sensor-Quell-Abständen zuzuordnen. Diese besitzen dann die Einheit Meter (m). 


\subsubsection{Schätzung auf der Basis instationärer Konzentrationen}

Die Schätzung der skalierbaren Sensor-Quell-Abstände auf der Basis stationärer Konzentrationen hat den Nachteil, dass erst lange Zeit nach Emissionsbeginn stationäre Konzentrationen vorliegen und damit erst spät eine Quellenlokalisierung möglich ist. Deshalb sollen die instationären Konzentrationen zum Ermitteln skalierbarer Sensor-Quell-Abstände herangezogen werden.

Entsprechend dem Ausbreitungsmodell (2.31) gilt für jeden Sensor $i$

$$
C\left(\mathbf{x}_{i}, t_{i, k}, \alpha_{i}, \beta_{i}, t_{0}\right)=\alpha_{i} \cdot \operatorname{erfc}\left(\frac{\beta_{i}}{\sqrt{t_{i, k}-t_{0}}}\right) \cdot 1\left(t_{i, k}-t_{0}\right)
$$

mit den zusammengefassten Größen

$$
\alpha_{i}=\frac{q_{0}}{4 \pi K\left\|\mathbf{x}_{i}-\mathbf{x}_{0}\right\|_{2}}, \quad \beta_{i}=\frac{\left\|\mathbf{x}_{i}-\mathbf{x}_{0}\right\|_{2}}{2 \sqrt{K}} .
$$

Gelingt es, $\alpha_{i}$ und $\beta_{i}$ für jeden Sensor $i$ auf der Basis der gemessenen instationären Konzentrationen $C_{i, k}$ zu schätzen, lassen sich daraus die skalierbaren Sensor-Quell-Abstände mit

$$
\hat{\varrho}_{i, \alpha}=\frac{1}{\hat{\alpha}_{i}} \quad \text { oder } \quad \hat{\varrho}_{i, \beta}=\hat{\beta}_{i}
$$

bestimmen. Die zugehörigen Skalierungsfaktoren sind dann

$$
\gamma_{\alpha}=\frac{4 \pi K}{q_{0}} \quad \text { oder } \quad \gamma_{\beta}=\frac{1}{2 \sqrt{K}} .
$$

Neben den Größen $\alpha_{i}$ und $\beta_{i}$ muss zusätzlich der unbekannte Startzeitpunkt der Emission $t_{0}$ ermittelt werden. Wegen der Nichtlinearität von (3.7) in $\alpha_{i}, \beta_{i}$ und $t_{0}$ bietet sich für die Schätzung an, den Fehler

$$
J_{i}=\sum_{k=1}^{N_{i}}\left(C_{i, k}-C\left(\mathbf{x}_{i}, t_{i, k}, \alpha_{i}, \beta_{i}, t_{0}\right)\right)^{2} \stackrel{!}{=} \operatorname{Min}_{\alpha_{i}, \beta_{i}, t_{0}}
$$

mit Hilfe von Gradientenverfahren zu minimieren.

Für die in Beispiel 1.2 gezeigten Konzentrationsverläufe für $t_{0}=0$ ergeben sich für die einzelnen Sensoren die in Abb. 3.3 dargestellten Gütegebirge über $t_{0}$, wenn $\alpha_{i}$ und $\beta_{i}$ auf ihre wahren Werte festgesetzt werden.

Anhand der Form des in Abb. 3.3 dargestellten Gütegebirges ist grundsätzlich ein gutes Konvergenzverhalten von Gradientenverfahren zu erwarten, da keine Nebenminima existieren. Es ist zudem zu erkennen, dass das Gütegebirge für den Sensor, der der Quelle am nächsten steht (Sensor 3), am besten ausgeprägt ist. Daher reicht es aus, die Startzeit 


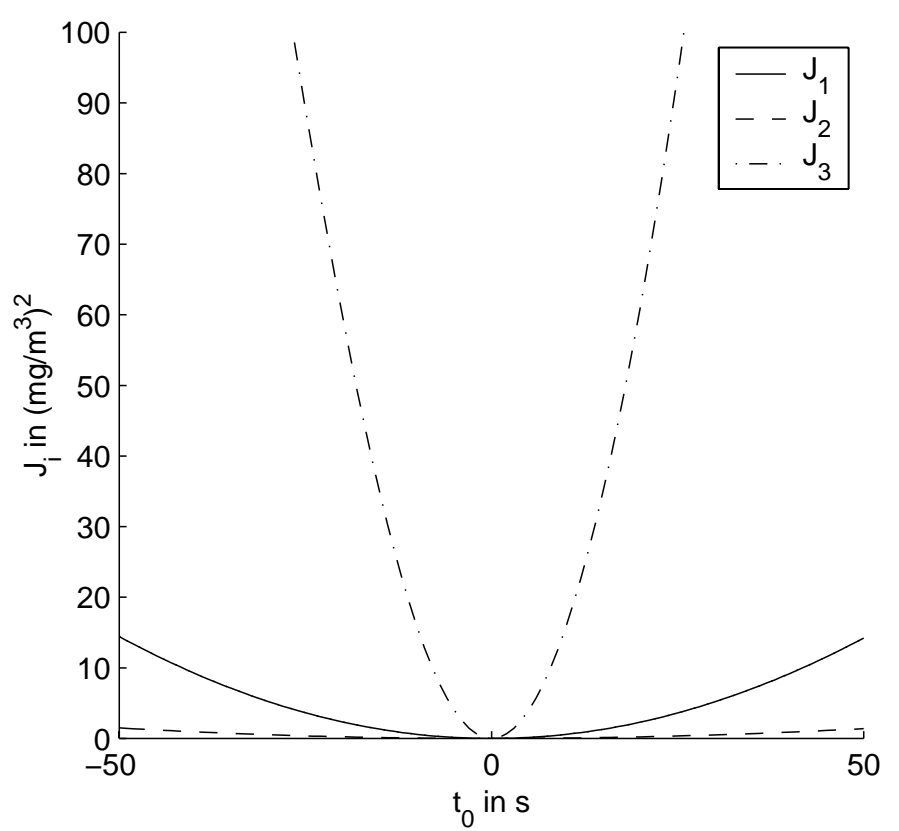

Abb. 3.3: Abhängigkeit von $J_{i}$ bezüglich der Startzeit $t_{0}$ für alle Sensoren $i$ gemäß Beispiel 1.2. (Die Sensoren 1 und 4 besitzen den gleichen Verlauf für $J_{i}$.)

nur auf der Basis desjenigen Sensors $j$ zu schätzen, der die größte Konzentration misst und damit den kleinsten Abstand zur Quelle besitzt.

Bei der Schätzung von $t_{0}$ ist zu beachten, dass der zeitabhängige Term in (3.7) mit zunehmender Zeit gegen den Wert 1 strebt

$$
\lim _{t \rightarrow \infty} \operatorname{erfc}\left(\beta_{i} \cdot \frac{1}{\sqrt{t-t_{0}}}\right) \cdot 1\left(t-t_{0}\right)=1 .
$$

Aus diesem Grund sinkt der Informationsgehalt für $t_{0}$ in Messungen $C_{i, k}$ mit zunehmender Messdauer. Die „persistent-excitation“-Bedingung ist nicht erfüllt [104]; die Schätzung für $t_{0}$ muss deshalb inkonsistent ${ }^{1}$ sein. Bei den zu erwartenden Störungen (unkorreliertes stationäres normalverteiltes Messrauschen) ist eine bleibende Abweichung zwischen Schätzwert $\hat{t}_{0}$ und wahrem Wert $t_{0}$ nicht zu vermeiden.

Anschließend werden die Parameter $\hat{\alpha}_{i}$ und $\hat{\beta}_{i}$ für jeden Sensor $i$ gemäß (3.11) geschätzt. Dabei wird jedoch der Wert für $t_{0}$ auf die Schätzung $\hat{t}_{0}$ festgesetzt. Abbildung 3.4 zeigt für jeden Sensor $i$ das Gütegebirge über $\alpha_{i}$ und $\beta_{i}$ auf der Basis von Beispiel 1.2. Es ist zu erkennen, dass neben den globalen Minima keine Nebenminima existieren. Aus diesem Grund sind hier bei der Anwendung von Gradientenverfahren keine Probleme zu erwarten, insbesondere wenn berücksichtigt wird, dass $\alpha_{i}$ und $\beta_{i}$ stets positiv sind.

\footnotetext{
${ }^{1}$ Eine Schätzung $\Gamma\left(X_{1}, \ldots, X_{n}\right)$ des Parameters $\gamma$ heißt konsistent, wenn $\Gamma\left(X_{1}, \ldots, X_{n}\right)$ in Wahrscheinlichkeit gegen den Parameter $\gamma$ konvergiert, d. h., wenn für beliebiges $\varepsilon>0 \lim _{n \rightarrow \infty} P\left(\left|\Gamma\left(X_{1}, \ldots, X_{n}\right)-\gamma\right| \leq\right.$ $\varepsilon)=1$ gilt. $[13]$
} 

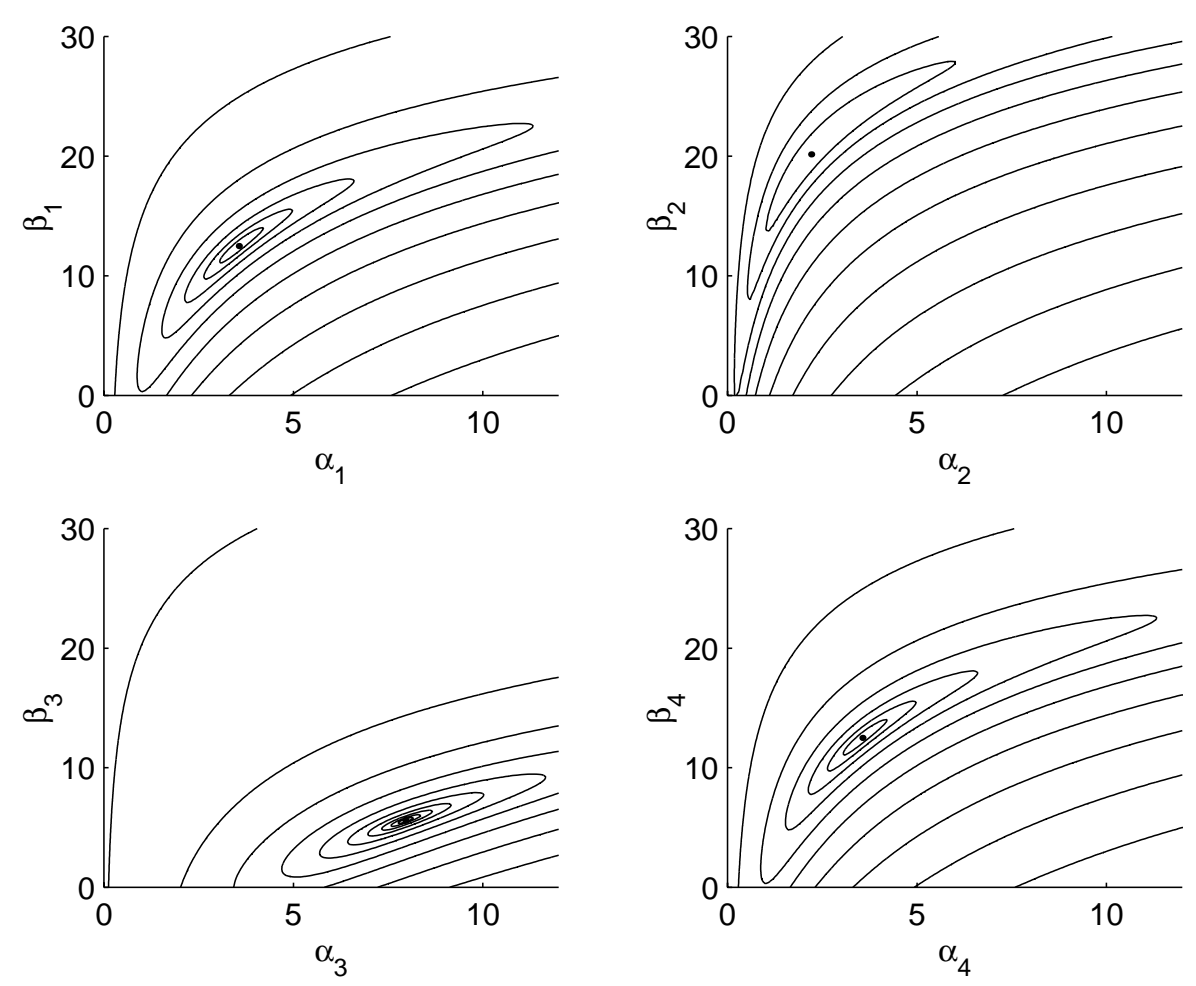

Abb. 3.4: Abhängigkeit von $J_{i}$ bezüglich $\alpha_{i}$ und $\beta_{i}$ für alle Sensoren $i$ gemäß Beispiel 1.2.

Wegen der Inkonsistenz der Schätzung von $\hat{t}_{0}$ ist auch die Schätzung von $\hat{\beta}_{i}$ inkonsistent, selbst wenn $\hat{t}_{0}=t_{0}$ gilt, da (3.12) eine Abnahme des Informationsgehalts bewirkt. Die Schätzung von $\hat{\alpha}_{i}$ verbessert sich hingegen mit zunehmender Beobachtungsdauer; sie ist konsistent. Aus diesem Grund wird $\hat{\alpha}_{i}$ zur Ermittlung skalierbarer Sensor-Quell-Abstände genutzt

$$
\hat{\varrho}_{i}=\hat{\varrho}_{i, \alpha}=\frac{1}{\hat{\alpha}_{i}} .
$$

Daraus folgt für den Skalierungsfaktor

$$
\gamma=\gamma_{\alpha}=\frac{4 \pi K}{q_{0}} \quad \text { bzw. } \quad \gamma=\gamma_{\alpha}=\frac{2 \pi K}{q_{0}} \quad \text { (für das halbunendliche Medium). }
$$

Während das Gütegebirge bezüglich des Quellorts (1.1), das bei einstufigen Verfahren zum Einsatz kommt, Nebenminima im Gütegebirge besitzt (vgl. Abb. 1.5), gelingt es durch den zweistufigen Ansatz zumindest skalierbare Sensor-Quell-Abstände für alle Sensoren eindeutig zu ermitteln. Dies wird besonders deutlich, wenn, wie für Abb. 1.5 vorausgesetzt, sowohl die Quellintensität $q_{0}$ als auch der Diffusionskoeffizient $K$ bekannt sind. Dann ist nämlich der Skalierungsfaktor $\gamma_{\alpha}$ bekannt, und es können direkt Sensor-Quell-Abstände $d_{i}=\left\|\mathbf{x}_{i}-\mathbf{x}_{0}\right\|_{2}$ geschätzt werden. Die zugehörigen Gütegebirge bezüglich $d_{i}$ auf der Basis von Beispiel 1.2 zeigt Abb. 3.5. Diese Gütegebirge sind für Gradientenverfahren unproblematisch. 

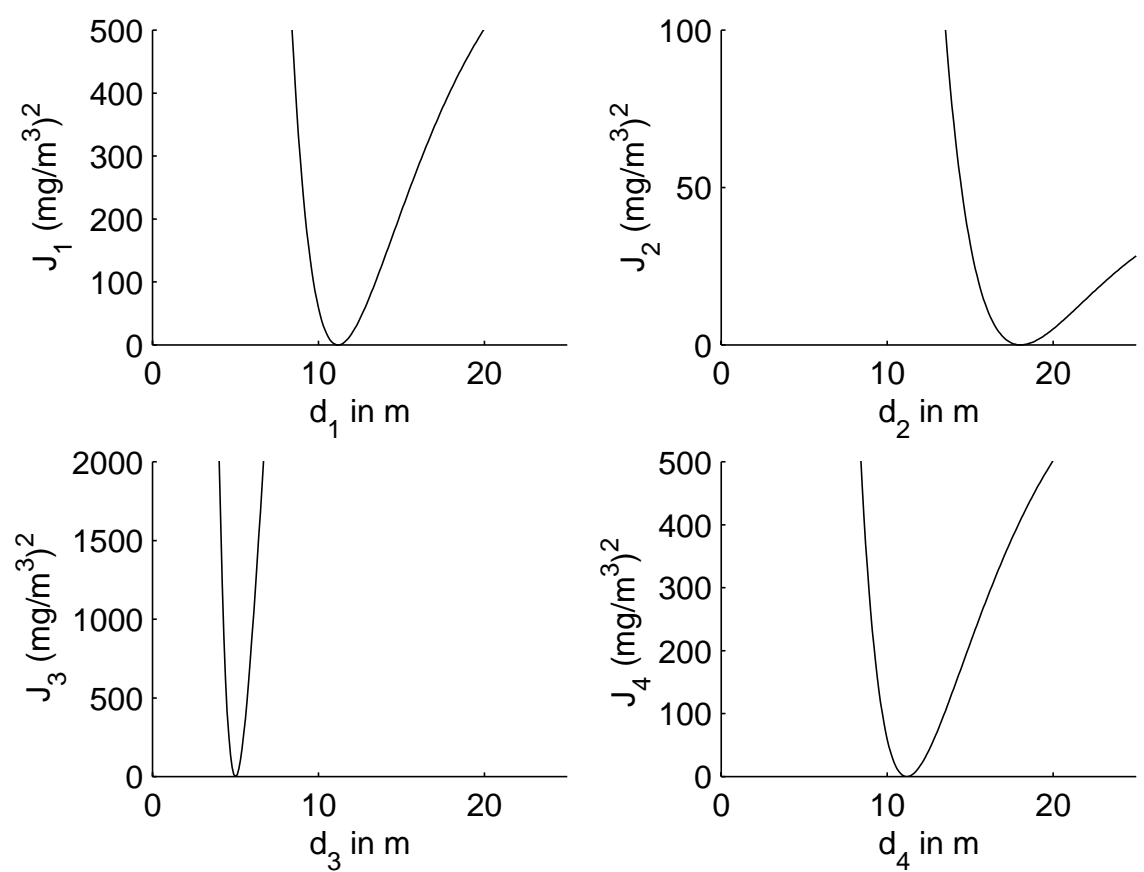

Abb. 3.5: Abhängigkeit von $J_{i}$ bezüglich $d_{i}$ für alle Sensoren $i$ gemäß Beispiel 1.2.

\subsubsection{Bewertung der Schätzverfahren}

In den vorangegangenen Abschnitten wurde gezeigt, wie sich die skalierbaren SensorQuell-Abstände sowohl auf der Basis stationärer als auch instationärer Konzentrationen ermitteln lassen. Die wichtigsten Eigenschaften der Verfahren fasst Tabelle 3.1 zusammen.

\begin{tabular}{|c|c|c|}
\hline & $\begin{array}{c}\text { Verfahren mit stationären } \\
\text { Konzentrationen }\end{array}$ & $\begin{array}{c}\text { Verfahren mit instationären } \\
\text { Konzentrationen }\end{array}$ \\
\hline \hline Schätzen von $t_{0}$ & nicht erforderlich & erforderlich \\
\hline Rechenaufwand & $\begin{array}{c}\text { gering } \\
\text { (Mittelwertbildung) }\end{array}$ & $\begin{array}{c}\text { (Optimierung mit } \\
\text { Gradientenverfahren) }\end{array}$ \\
\hline $\begin{array}{c}\text { Verhalten bei } \\
\text { Störungen/Rauschen }\end{array}$ & $\begin{array}{c}\text { robust } \\
\text { (durch Mittelwertbildung) }\end{array}$ & $\begin{array}{c}\text { (Ausgleich über alle Mess- } \\
\text { werte bei Optimierung) }\end{array}$ \\
\hline $\begin{array}{c}\text { möglicher Zeitpunkt } \\
\text { der Ergebnisausgabe }\end{array}$ & $\begin{array}{c}\text { noch drei } \\
\text { Erreichen stationärer } \\
\text { Konzentrationen }\end{array}$ & $\begin{array}{c}\text { Konzentrationsmessungen } \\
\text { pro Sensor (drei Parameter } \\
\text { pro Sensor) }\end{array}$ \\
\hline $\begin{array}{c}\text { Konsistenz der } \\
\text { Schätzung }\end{array}$ & konsistent & konsistent \\
\hline
\end{tabular}

Tabelle 3.1: Eigenschaften der Verfahren zur Ermittlung skalierbarer Sensor-Quell-Abstände. 


\subsection{Ermittlung des Quellorts im ungestörten Fall}

\subsubsection{Vorbemerkungen}

Um den Quellort $\mathbf{x}_{0}$ aus den skalierbaren Sensor-Quell-Abständen $\hat{\varrho}_{i}$ zu berechnen, ist das Kugelschnittproblem

$$
\hat{\varrho}_{i}=\gamma \cdot\left\|\mathbf{x}_{i}-\mathbf{x}_{0}\right\|_{2} \quad i=1, \ldots, p
$$

zu lösen. Dabei spielt es keine Rolle, ob $\hat{\varrho}_{i}$ auf der Basis stationärer oder instationärer Konzentrationen geschätzt wurde.

In diesem und im nächsten Abschnitt wird zunächst der ungestörte Fall behandelt. Die geschätzten skalierbaren Sensor-Quell-Abstände sind gleich den wahren Werten, und anstelle von $\hat{\varrho}_{i}$ lässt sich $\varrho_{i}$ benutzen.

Bei den Untersuchungen werden vier Sensor-Quell-Konfigurationen unterschieden.

- Linienkonfiguration: die Quelle und alle Sensoren befinden sich auf einer Geraden. (Eine solche Konfiguration ist praktisch beispielsweise bei der Überwachung einer Rohrleitung denkbar, wenn alle Sensoren auf der Rohrleitung montiert sind.)

- Ebenenkonfiguration: die Quelle und alle Sensoren befinden sich auf einer Ebene. (Eine Ebenenkonfiguration tritt beispielsweise dann auf, wenn der Quellort und alle Sensoren auf dem Boden liegen.)

- Raumkonfiguration: die Quelle und alle Sensoren sind beliebig im unendlichen Ausbreitungsmedium verteilt. (Eine Raumkonfiguration stellt z. B. ein Hochregallager dar, in dem auf mehreren Ebenen Sensoren installiert sind.)

- Halbraumkonfiguration: die Quelle befindet sich auf der impermeablen Grenzfläche eines halbunendlichen Ausbreitungsmediums, und die Sensoren sind frei im Halbraum verteilt. (Eine Halbraumkonfiguration liegt beispielsweise dann vor, wenn eine tropfende Leckage immer zu einem Quellort am Boden (impermeabel) führt, sich die Sensoren aber nicht auf dem Boden befinden.)

Für den ungestörten Fall wird für die einzelnen Sensor-Quell-Konfigurationen versucht, den Quellort $\mathbf{x}_{0}$ und den Skalierungsfaktor $\gamma$ mit minimaler Anzahl von Sensoren zu ermitteln. Die Untersuchungen für die minimale Sensoranzahl erlauben es, Aussagen zur Existenz und Eindeutigkeit von Lösungen sowie zur geeigneten Wahl von Sensorstandorten zu treffen. Im Abschnitt 3.4 werden die gewonnenen Ergebnisse auf den gestörten Fall mit beliebiger Sensoranzahl $p$ erweitert. 


\subsubsection{Linienkonfiguration}

Bei der Linienkonfiguration liegen alle Sensoren und die Quelle auf einer Geraden $\left(\mathbf{x}_{i}=x_{i}\right.$ und $\mathbf{x}_{0}=x_{0}$ ). Es sind die Parameter Quellort $x_{0}$ und Skalierungsfaktor $\gamma$ zu bestimmen. Mit den dazu minimal notwendigen zwei Sensoren bei $x_{1}$ und $x_{2}\left(x_{1}<x_{2}\right)$ sowie den zugehörigen Sensor-Quell-Abständen $\varrho_{1}$ und $\varrho_{2}$ lässt sich für die gesuchten Parameter ein Gleichungssystem

$$
\begin{aligned}
& \varrho_{1}=\gamma \cdot\left|x_{1}-x_{0}\right| \\
& \varrho_{2}=\gamma \cdot\left|x_{2}-x_{0}\right|
\end{aligned}
$$

aufstellen. Um dieses System zu lösen, müssen für das Auflösen der Beträge drei Fälle unterschieden werden.

$$
\begin{array}{lll}
x_{1}<x_{0}<x_{2}: & x_{0, I}=\frac{\varrho_{1} \cdot x_{2}+\varrho_{2} \cdot x_{1}}{\varrho_{1}+\varrho_{2}}, & \gamma_{I}=\frac{\varrho_{1}+\varrho_{2}}{x_{2}-x_{1}} \\
x_{0}<x_{1}<x_{2}: & x_{0, I I}=\frac{\varrho_{1} \cdot x_{2}-\varrho_{2} \cdot x_{1}}{\varrho_{1}-\varrho_{2}}, & \gamma_{I I}=\frac{\varrho_{1}-\varrho_{2}}{x_{2}-x_{1}} \\
x_{1}<x_{2}<x_{0}: & x_{0, I I I}=\frac{\varrho_{1} \cdot x_{2}-\varrho_{2} \cdot x_{1}}{\varrho_{1}-\varrho_{2}}, & \gamma_{I I I}=\frac{\varrho_{1}-\varrho_{2}}{x_{2}-x_{1}} .
\end{array}
$$

Es ist zu erkennen, dass die Lösungen $x_{0, I I}$ und $x_{0, I I I}$ identisch sind. Die Lage der Lösung $x_{0}<x_{1}$ bzw. $x_{2}<x_{0}$ ergibt sich automatisch wegen $\varrho_{1}<\varrho_{2}$ bzw. $\varrho_{1}>\varrho_{2}$. Im Fall $\varrho_{1}=\varrho_{2}$ existiert nur die Lösung $x_{0, I}$, die sich dann in der Mitte zwischen beiden Sensorstandorten $x_{1}$ und $x_{2}$ befindet.

Bei Verwenden der minimalen Sensoranzahl wird also neben dem wahren Quellort $x_{0, I}$ zusätzlich ein scheinbarer Quellort $x_{0, I I}$ erhalten. Wie diese zwei Lösungen zustande kommen, wird anhand stationärer Konzentrationen in Beispiel 3.1 gezeigt.

Beispiel 3.1 Bei eindimensionaler Sensor-Quell-Anordnung mit $x_{1}=10 \mathrm{~m}, x_{2}=20 \mathrm{~m}$ soll die Quelle bei $x_{0}=16 \mathrm{~m}$ mit der Intensität $q_{0}=1.26 \mathrm{mg} / \mathrm{s}$ anhand der Konzentrationen $C\left(x_{1}, \infty\right)=0.167 \mathrm{mg} / \mathrm{m}^{3}, C\left(x_{2}, \infty\right)=0.250 \mathrm{mg} / \mathrm{m}^{3}$ lokalisiert werden.

Aus (3.5) folgen die skalierbaren Sensor-Quell-Abstände $\varrho_{1}=6 \mathrm{~m}$ und $\varrho_{2}=4 \mathrm{~m}$. Mit (3.17) werden sowohl der wahre Quellort $x_{0, I}=x_{0}=16 \mathrm{~m}$ mit dem Skalierungsfaktor $\gamma_{I}=1$ und $q_{0, I}=1.26 \mathrm{mg} / \mathrm{s}$ als auch der scheinbare Quellort $x_{0, I I}=40 \mathrm{~m}$ mit $\gamma_{I I}=0.2$ und $q_{0, I I}=6.28 \mathrm{mg} / \mathrm{s}$ erhalten.

Abbildung 3.6 zeigt die stationären Konzentrationsprofile für den wahren und den scheinbaren Quellort gemäß Beispiel 3.1. 


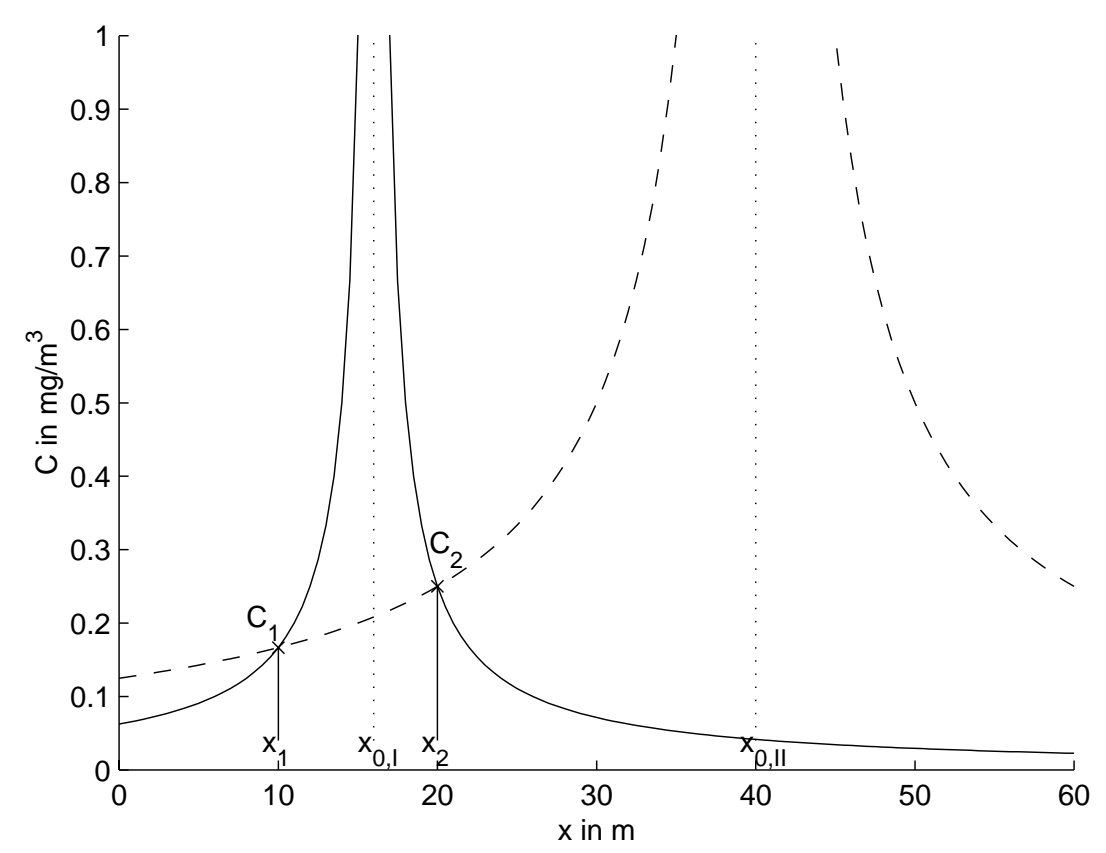

Abb. 3.6: Konzentrationsprofile für wahren und scheinbaren Quellort.

Es ist zu erkennen, dass an den Sensorstandorten beide Konzentrationsprofile identische Werte besitzen und damit anhand der Messungen nicht zu unterscheiden sind. Der wahre Quellort lässt sich nur durch Hinzunahme eines dritten Sensors bestimmen.

\subsubsection{Ebenenkonfiguration}

Bei der Ebenenkonfiguration liegen alle Sensoren und die Quelle auf einer Ebene $\left(\mathbf{x}_{i}=\right.$ $\left.\left(x_{i}, y_{i}\right)^{T}, \mathbf{x}_{0}=\left(x_{0}, y_{0}\right)^{T}\right)$. Zum Ermitteln der unbekannten Parameter $x_{0}, y_{0}$ und $\gamma$ sind mindestens drei Sensoren notwendig. Mit den zugehörigen skalierbaren Sensor-Quell-Abständen ergibt sich

$$
\varrho_{i}=\gamma \cdot\left\|\mathbf{x}_{i}-\mathbf{x}_{0}\right\|_{2} \quad i=1, \ldots, 3
$$

Durch Eliminieren von $\gamma$ wird

$$
\begin{aligned}
& \varrho_{2} \cdot\left\|\mathbf{x}_{1}-\mathbf{x}_{0}\right\|_{2}=\varrho_{1} \cdot\left\|\mathbf{x}_{2}-\mathbf{x}_{0}\right\|_{2} \\
& \varrho_{3} \cdot\left\|\mathbf{x}_{1}-\mathbf{x}_{0}\right\|_{2}=\varrho_{1} \cdot\left\|\mathbf{x}_{3}-\mathbf{x}_{0}\right\|_{2}
\end{aligned}
$$

erhalten. Quadrieren und Aufösen der euklidischen Norm führt auf das algebraische Gleichungssystem ${ }^{2}$

$$
\begin{aligned}
& \varrho_{2}^{2} \cdot\left(\mathbf{x}_{1}^{T} \mathbf{x}_{1}-2 \mathbf{x}_{1}^{T} \mathbf{x}_{0}+\mathbf{x}_{0}^{T} \mathbf{x}_{0}\right)=\varrho_{1}^{2} \cdot\left(\mathbf{x}_{2}^{T} \mathbf{x}_{2}-2 \mathbf{x}_{2}^{T} \mathbf{x}_{0}+\mathbf{x}_{0}^{T} \mathbf{x}_{0}\right) \\
& \varrho_{3}^{2} \cdot\left(\mathbf{x}_{1}^{T} \mathbf{x}_{1}-2 \mathbf{x}_{1}^{T} \mathbf{x}_{0}+\mathbf{x}_{0}^{T} \mathbf{x}_{0}\right)=\varrho_{1}^{2} \cdot\left(\mathbf{x}_{3}^{T} \mathbf{x}_{3}-2 \mathbf{x}_{3}^{T} \mathbf{x}_{0}+\mathbf{x}_{0}^{T} \mathbf{x}_{0}\right)
\end{aligned}
$$

\footnotetext{
${ }^{2}$ Ein System von Gleichungen $P_{1}(x, y, z, \ldots)=0, P_{2}(x, y, z, \ldots)=0, \ldots, P_{n}(x, y, z, \ldots)=0$, wobei $P_{i}(x, y, z, \ldots)$ Polynome in $x, y, z, \ldots$ sind, heißt algebraisches Gleichungssystem [14].
} 


\section{Lösung mittels Gröbner-Basis}

Im Gegensatz zur Linienkonfiguration lässt sich das algebraische Gleichungssystem (3.18) bzw. (3.20) nicht ohne weiteres lösen. Zum Lösen bietet es sich an, eine Gröbner-Basis ${ }^{3}$ $\mathrm{zu}(3.20) \mathrm{zu}$ ermitteln.

Hier wird der in MAPLE angebotene Algorithmus verwendet, um eine Gröbner-Basis für (3.20) zu ermitteln. Dies liefert die (sehr unhandliche) Lösung

$$
\begin{aligned}
& a_{1} \cdot y_{0}^{2}+a_{2} \cdot y_{0}+a_{3}=0 \\
& a_{4} \cdot x_{0}+a_{5} \cdot y_{0}+a_{6}=0
\end{aligned}
$$

mit den Koeffizienten

$$
\begin{aligned}
a_{1}= & 4 \varrho_{2}^{4} y_{1}^{2}+4 \varrho_{2}^{4} x_{1}^{2}+4 \varrho_{2}^{4} y_{3}^{2}+4 \varrho_{2}^{4} x_{3}^{2}-8 \varrho_{3}^{2} \varrho_{2}^{2} x_{1}^{2}-8 \varrho_{3}^{2} \varrho_{2}^{2} y_{1}^{2}-8 \varrho_{3}^{2} \varrho_{1}^{2} x_{2}^{2}-8 \varrho_{3}^{2} \varrho_{1}^{2} y_{2}^{2}-8 \varrho_{2}^{2} \varrho_{1}^{2} x_{3}^{2}- \\
& 8 \varrho_{2}^{2} \varrho_{1}^{2} y_{3}^{2}+4 \varrho_{1}^{4} x_{2}^{2}+4 \varrho_{1}^{4} y_{2}^{2}+4 \varrho_{1}^{4} x_{3}^{2}+4 \varrho_{1}^{4} y_{3}^{2}+8 \varrho_{3}^{2} x_{2} \varrho_{1}^{2} x_{3}+8 \varrho_{1}^{2} x_{2} \varrho_{3}^{2} x_{1}+8 \varrho_{2}^{2} x_{1} \varrho_{1}^{2} x_{3}+ \\
& 8 \varrho_{2}^{2} x_{3} \varrho_{3}^{2} x_{1}-8 \varrho_{1}^{2} x_{3} \varrho_{3}^{2} x_{1}-8 \varrho_{3}^{4} x_{2} x_{1}-8 \varrho_{1}^{4} x_{2} x_{3}+4 \varrho_{3}^{4} x_{1}^{2}+4 \varrho_{3}^{4} x_{2}^{2}+4 \varrho_{3}^{4} y_{2}^{2}+4 \varrho_{3}^{4} y_{1}^{2}-8 \varrho_{3}^{4} y_{2} y_{1}- \\
& 8 \varrho_{2}^{4} x_{1} x_{3}-8 \varrho_{2}^{4} y_{1} y_{3}-8 \varrho_{1}^{4} y_{2} y_{3}+8 \varrho_{3}^{2} y_{2} \varrho_{2}^{2} y_{1}-8 \varrho_{3}^{2} y_{2} \varrho_{2}^{2} y_{3}+8 \varrho_{3}^{2} x_{2} \varrho_{2}^{2} x_{1}+8 \varrho_{3}^{2} \varrho_{2}^{2} y_{1} y_{3}+ \\
& 8 \varrho_{1}^{2} \varrho_{3}^{2} y_{2} y_{3}-8 \varrho_{3}^{2} x_{2} \varrho_{2}^{2} x_{3}-8 \varrho_{1}^{2} x_{2} \varrho_{2}^{2} x_{1}+8 \varrho_{1}^{2} x_{2} \varrho_{2}^{2} x_{3}+8 \varrho_{1}^{2} y_{2} \varrho_{2}^{2} y_{3}+8 \varrho_{1}^{2} y_{2} \varrho_{3}^{2} y_{1}+8 \varrho_{1}^{2} \varrho_{2}^{2} y_{1} y_{3}- \\
& 8 \varrho_{1}^{2} \varrho_{3}^{2} y_{1} y_{3}-8 \varrho_{1}^{2} y_{2} \varrho_{2}^{2} y_{1}
\end{aligned}
$$$$
a_{2}=-4 \varrho_{1}^{2} \varrho_{3}^{2} y_{2} y_{3}^{2}+4 \varrho_{1}^{2} x_{3}^{2} \varrho_{3}^{2} y_{1}+4 \varrho_{1}^{2} x_{2}^{2} \varrho_{3}^{2} y_{1}+4 \varrho_{1}^{2} \varrho_{3}^{2} x_{2}^{2} y_{3}+8 \varrho_{1}^{2} \varrho_{3}^{2} y_{2} x_{2}^{2}-4 \varrho_{3}^{2} y_{3} \varrho_{2}^{2} y_{1}^{2}-4 \varrho_{3}^{2} \varrho_{2}^{2} y_{1} y_{3}^{2}+
$$$$
4 \varrho_{3}^{2} y_{2} \varrho_{2}^{2} x_{3}^{2}+4 \varrho_{3}^{2} y_{2} \varrho_{2}^{2} x_{1}^{2}+8 \varrho_{2}^{2} y_{1} \varrho_{3}^{2} x_{1}^{2}+8 \varrho_{2}^{4} x_{1} x_{3} y_{1}+8 x_{2} \varrho_{3}^{4} x_{1} y_{2}+4 \varrho_{3}^{2} \varrho_{2}^{2} y_{3} y_{2}^{2}+4 \varrho_{3}^{2} \varrho_{2}^{2} y_{3} x_{2}^{2}-
$$$$
4 \varrho_{3}^{2} \varrho_{2}^{2} y_{1} y_{2}^{2}-4 \varrho_{3}^{2} \varrho_{2}^{2} y_{1} x_{2}^{2}-4 \varrho_{2}^{2} x_{3}^{2} \varrho_{3}^{2} y_{1}+4 \varrho_{2}^{2} x_{1}^{2} \varrho_{3}^{2} y_{3}-4 \varrho_{2}^{4} y_{1} x_{1}^{2}+8 \varrho_{2}^{4} x_{1} x_{3} y_{3}+8 x_{2} \varrho_{3}^{4} x_{1} y_{1}+
$$$$
4 \varrho_{3}^{2} y_{2} \varrho_{2}^{2} y_{3}^{2}-4 \varrho_{1}^{4} y_{3}^{3}-4 \varrho_{1}^{4} y_{2}^{3}-4 \varrho_{3}^{4} y_{1}^{3}-4 \varrho_{2}^{4} y_{1}^{3}+8 \varrho_{1}^{4} x_{2} x_{3} y_{2}-4 \varrho_{2}^{4} y_{3}^{3}-4 \varrho_{3}^{4} y_{2}^{3}-8 \varrho_{3}^{2} x_{2} \varrho_{2}^{2} x_{1} y_{3}-
$$$$
4 \varrho_{1}^{2} \varrho_{3}^{2} y_{2} x_{3}^{2}+4 \varrho_{1}^{2} y_{3} \varrho_{3}^{2} y_{1}^{2}+4 \varrho_{1}^{2} y_{3} \varrho_{3}^{2} x_{1}^{2}-4 \varrho_{3}^{2} y_{2} \varrho_{2}^{2} y_{1}^{2}-4 \varrho_{1}^{2} y_{3} \varrho_{2}^{2} y_{1}^{2}-4 \varrho_{1}^{2} y_{3} \varrho_{2}^{2} x_{1}^{2}+4 \varrho_{1}^{2} \varrho_{3}^{2} y_{1} y_{3}^{2}-
$$$$
4 \varrho_{1}^{2} \varrho_{3}^{2} y_{1} y_{2}^{2}-4 \varrho_{1}^{2} \varrho_{2}^{2} y_{3} y_{2}^{2}-4 \varrho_{1}^{2} \varrho_{2}^{2} y_{3} x_{2}^{2}-4 \varrho_{1}^{2} \varrho_{2}^{2} y_{1} y_{3}^{2}+4 \varrho_{1}^{2} \varrho_{2}^{2} y_{1} x_{3}^{2}+4 \varrho_{1}^{2} \varrho_{2}^{2} y_{1} y_{2}^{2}+4 \varrho_{1}^{2} \varrho_{2}^{2} y_{1} x_{2}^{2}-
$$$$
4 \varrho_{1}^{2} y_{2} \varrho_{3}^{2} y_{1}^{2}-4 \varrho_{1}^{2} y_{2} \varrho_{3}^{2} x_{1}^{2}-4 \varrho_{1}^{2} y_{2} \varrho_{2}^{2} y_{3}^{2}+4 \varrho_{1}^{2} y_{2} \varrho_{2}^{2} x_{3}^{2}+4 \varrho_{1}^{2} y_{2} \varrho_{2}^{2} y_{1}^{2}+4 \varrho_{1}^{2} y_{2} \varrho_{2}^{2} x_{1}^{2}-4 \varrho_{1}^{2} \varrho_{3}^{2} y_{3} y_{2}^{2}-
$$$$
4 \varrho_{1}^{4} y_{2} x_{3}^{2}-4 \varrho_{1}^{4} y_{2} x_{2}^{2}-4 \varrho_{1}^{4} x_{3}^{2} y_{3}-4 \varrho_{1}^{4} x_{2}^{2} y_{3}+8 \varrho_{1}^{2} \varrho_{2}^{2} y_{3}^{3}+8 \varrho_{1}^{2} \varrho_{3}^{2} y_{2}^{3}-4 \varrho_{3}^{4} y_{2} x_{1}^{2}-4 \varrho_{3}^{4} y_{1} x_{1}^{2}+
$$$$
4 \varrho_{2}^{4} y_{3} y_{1}^{2}+8 \varrho_{2}^{2} y_{1}^{3} \varrho_{3}^{2}+4 \varrho_{2}^{4} y_{1} y_{3}^{2}-4 \varrho_{2}^{4} y_{1} x_{3}^{2}+4 \varrho_{3}^{4} y_{1} y_{2}^{2}+4 \varrho_{3}^{4} y_{2} y_{1}^{2}-4 \varrho_{2}^{4} x_{3}^{2} y_{3}-4 \varrho_{2}^{4} x_{1}^{2} y_{3}+
$$$$
16 \varrho_{1}^{2} x_{2} \varrho_{2}^{2} x_{1} y_{3}+8 \varrho_{1}^{2} \varrho_{2}^{2} x_{3}^{2} y_{3}-4 \varrho_{3}^{4} x_{2}^{2} y_{1}-8 \varrho_{3}^{2} \varrho_{2}^{2} x_{1} x_{3} y_{2}-8 \varrho_{2}^{2} x_{1} x_{3} \varrho_{3}^{2} y_{1}+16 x_{2} \varrho_{2}^{2} x_{3} \varrho_{3}^{2} y_{1}-
$$$$
4 \varrho_{3}^{4} y_{2} x_{2}^{2}-8 x_{2} \varrho_{2}^{2} x_{1} \varrho_{3}^{2} y_{1}+16 \varrho_{1}^{2} x_{3} \varrho_{3}^{2} x_{1} y_{2}-8 \varrho_{1}^{2} \varrho_{2}^{2} x_{1} x_{3} y_{2}-8 \varrho_{1}^{2} x_{2} \varrho_{3}^{2} x_{1} y_{2}+4 \varrho_{1}^{4} y_{3} y_{2}^{2}-
$$$$
8 \varrho_{1}^{2} \varrho_{3}^{2} x_{2} x_{3} y_{2}-8 \varrho_{1}^{2} \varrho_{2}^{2} x_{1} x_{3} y_{3}+4 \varrho_{1}^{4} y_{2} y_{3}^{2}-8 \varrho_{1}^{2} x_{2} \varrho_{3}^{2} x_{1} y_{3}-8 \varrho_{1}^{2} x_{2} x_{3} \varrho_{3}^{2} y_{1}-8 \varrho_{1}^{2} x_{2} \varrho_{2}^{2} x_{3} y_{3}-
$$$$
8 \varrho_{1}^{2} x_{2} x_{3} \varrho_{2}^{2} y_{1}+8 \varrho_{1}^{4} x_{2} x_{3} y_{3}
$$

\footnotetext{
${ }^{3}$ Eine Gröbner-Basis für ein System von Polynomen ist ein Äquivalenzsystem, das einige besondere Eigenschaften besitzt. Eine wesentliche Eigenschaft einer Gröbner-Basis besteht darin, dass die darin enthaltenen Polynome die gleichen Wurzeln besitzen wie die Originalpolynome. Für lineare Funktionen ist die Gröbner-Basis äquivalent zur Gauß-Elimination. Die Berechnung von Gröbner-Basen erfolgt mit dem Buchberger-Algorithmus, der in den meisten Computeralgebra-Systemen integriert ist. [1, 15]
} 


$$
\begin{aligned}
& a_{3}=-2 \varrho_{1}^{2} \varrho_{2}^{2} y_{3}^{4}+2 \varrho_{1}^{4} x_{2}^{2} y_{2}^{2}-2 \varrho_{1}^{4} y_{2}^{2} y_{3}^{2}+2 \varrho_{1}^{4} x_{2}^{2} y_{3}^{2}-4 \varrho_{1}^{4} x_{2}^{3} x_{3}+6 \varrho_{3}^{4} x_{2}^{2} x_{1}^{2}+2 \varrho_{3}^{4} x_{2}^{2} y_{1}^{2}-4 x_{2} \varrho_{3}^{4} x_{1}^{3}- \\
& 4 \varrho_{2}^{4} x_{1} x_{3}^{3}+6 \varrho_{2}^{4} x_{1}^{2} x_{3}^{2}+2 \varrho_{2}^{4} x_{1}^{2} y_{3}^{2}+2 \varrho_{2}^{4} x_{3}^{2} y_{3}^{2}+2 \varrho_{3}^{4} x_{2}^{2} y_{2}^{2}-2 \varrho_{3}^{4} y_{2}^{2} y_{1}^{2}+2 \varrho_{2}^{4} x_{1}^{2} y_{1}^{2}-2 \varrho_{2}^{2} x_{1}^{4} \varrho_{3}^{2}+ \\
& 2 \varrho_{2}^{4} y_{1}^{2} x_{3}^{2}-2 \varrho_{2}^{4} y_{1}^{2} y_{3}^{2}-2 \varrho_{2}^{2} y_{1}^{4} \varrho_{3}^{2}+2 \varrho_{3}^{4} x_{1}^{2} y_{1}^{2}-4 x_{2}^{3} \varrho_{3}^{4} x_{1}-4 \varrho_{2}^{4} x_{1}^{3} x_{3}+\varrho_{2}^{4} x_{3}^{4}+\varrho_{3}^{4} x_{2}^{4}+\varrho_{3}^{4} y_{2}^{4}+ \\
& \varrho_{2}^{4} x_{1}^{4}+\varrho_{2}^{4} y_{1}^{4}+\varrho_{2}^{4} y_{3}^{4}+\varrho_{3}^{4} x_{1}^{4}+\varrho_{3}^{4} y_{1}^{4}+\varrho_{1}^{4} y_{3}^{4}+\varrho_{1}^{4} x_{2}^{4}+\varrho_{1}^{4} y_{2}^{4}+\varrho_{1}^{4} x_{3}^{4}+4 x_{2} \varrho_{2}^{2} x_{1}^{3} \varrho_{3}^{2}-4 x_{2} \varrho_{3}^{4} x_{1} y_{1}^{2}- \\
& 4 \varrho_{2}^{4} x_{1} x_{3} y_{3}^{2}+4 \varrho_{2}^{2} x_{1}^{3} x_{3} \varrho_{3}^{2}-2 \varrho_{2}^{2} x_{1}^{2} \varrho_{3}^{2} y_{3}^{2}+2 \varrho_{2}^{2} x_{3}^{2} \varrho_{3}^{2} y_{1}^{2}+4 \varrho_{3}^{2} x_{2} \varrho_{2}^{2} x_{1} x_{3}^{2}+4 \varrho_{3}^{2} x_{2} \varrho_{2}^{2} x_{1} y_{3}^{2}+ \\
& 4 x_{2} \varrho_{2}^{2} x_{1} \varrho_{3}^{2} y_{1}^{2}-8 x_{2} \varrho_{2}^{2} x_{3} \varrho_{3}^{2} x_{1}^{2}-8 x_{2} \varrho_{2}^{2} x_{3} \varrho_{3}^{2} y_{1}^{2}+4 \varrho_{2}^{2} x_{1} x_{3} \varrho_{3}^{2} y_{1}^{2}+2 \varrho_{3}^{2} \varrho_{2}^{2} y_{1}^{2} x_{2}^{2}+2 \varrho_{3}^{2} \varrho_{2}^{2} y_{1}^{2} y_{2}^{2}- \\
& 2 \varrho_{3}^{2} x_{2}^{2} \varrho_{2}^{2} y_{3}^{2}-2 \varrho_{3}^{2} y_{2}^{2} \varrho_{2}^{2} y_{3}^{2}-4 \varrho_{2}^{2} x_{1}^{2} \varrho_{3}^{2} y_{1}^{2}-4 x_{2} \varrho_{3}^{4} x_{1} y_{2}^{2}-4 \varrho_{2}^{4} x_{1} x_{3} y_{1}^{2}+2 \varrho_{3}^{2} \varrho_{2}^{2} y_{1}^{2} y_{3}^{2}-2 \varrho_{3}^{2} \varrho_{2}^{2} x_{1}^{2} x_{2}^{2}- \\
& 2 \varrho_{3}^{2} \varrho_{2}^{2} x_{1}^{2} y_{2}^{2}-2 \varrho_{3}^{2} \varrho_{2}^{2} x_{1}^{2} x_{3}^{2}-2 \varrho_{3}^{2} \varrho_{2}^{2} x_{3}^{2} x_{2}^{2}-2 \varrho_{3}^{2} \varrho_{2}^{2} x_{3}^{2} y_{2}^{2}+2 \varrho_{3}^{4} x_{1}^{2} y_{2}^{2}+4 \varrho_{3}^{2} \varrho_{2}^{2} x_{1} x_{3} x_{2}^{2}+4 \varrho_{3}^{2} \varrho_{2}^{2} x_{1} x_{3} y_{2}^{2}- \\
& 2 \varrho_{1}^{2} \varrho_{3}^{2} x_{2}^{4}-2 \varrho_{1}^{2} \varrho_{3}^{2} y_{2}^{4}-2 \varrho_{1}^{2} \varrho_{2}^{2} x_{3}^{4}-4 \varrho_{1}^{4} x_{2} x_{3}^{3}+2 \varrho_{1}^{4} x_{3}^{2} y_{3}^{2}+2 \varrho_{1}^{4} y_{2}^{2} x_{3}^{2}+6 \varrho_{1}^{4} x_{2}^{2} x_{3}^{2}+2 \varrho_{1}^{2} \varrho_{3}^{2} y_{2}^{2} x_{3}^{2}- \\
& 2 \varrho_{1}^{2} \varrho_{3}^{2} x_{2}^{2} x_{3}^{2}-2 \varrho_{1}^{2} \varrho_{3}^{2} x_{2}^{2} y_{3}^{2}-2 \varrho_{1}^{2} \varrho_{3}^{2} y_{3}^{2} y_{1}^{2}+2 \varrho_{1}^{2} \varrho_{2}^{2} y_{1}^{2} y_{3}^{2}-2 \varrho_{1}^{2} \varrho_{3}^{2} x_{2}^{2} y_{1}^{2}-4 \varrho_{1}^{2} \varrho_{3}^{2} x_{2}^{2} y_{2}^{2}+2 \varrho_{1}^{2} \varrho_{3}^{2} y_{2}^{2} y_{1}^{2}- \\
& 2 \varrho_{1}^{2} \varrho_{3}^{2} x_{1}^{2} y_{3}^{2}-2 \varrho_{1}^{2} \varrho_{3}^{2} x_{2}^{2} x_{1}^{2}+2 \varrho_{1}^{2} \varrho_{2}^{2} x_{1}^{2} y_{3}^{2}+4 \varrho_{1}^{2} x_{2}^{3} \varrho_{3}^{2} x_{1}+2 \varrho_{1}^{2} \varrho_{3}^{2} x_{1}^{2} y_{2}^{2}+2 \varrho_{1}^{2} \varrho_{3}^{2} y_{3}^{2} y_{2}^{2}+4 \varrho_{1}^{2} x_{2} \varrho_{2}^{2} x_{3}^{3}+ \\
& 4 \varrho_{1}^{2} \varrho_{2}^{2} x_{1} x_{3}^{3}-4 \varrho_{1}^{2} \varrho_{2}^{2} x_{3}^{2} y_{3}^{2}-2 \varrho_{1}^{2} x_{3}^{2} \varrho_{3}^{2} x_{1}^{2}-2 \varrho_{1}^{2} x_{3}^{2} \varrho_{3}^{2} y_{1}^{2}-2 \varrho_{1}^{2} \varrho_{2}^{2} x_{1}^{2} x_{2}^{2}-2 \varrho_{1}^{2} \varrho_{2}^{2} x_{1}^{2} y_{2}^{2}-2 \varrho_{1}^{2} \varrho_{2}^{2} x_{1}^{2} x_{3}^{2}- \\
& 2 \varrho_{1}^{2} \varrho_{2}^{2} y_{1}^{2} x_{2}^{2}-2 \varrho_{1}^{2} \varrho_{2}^{2} y_{1}^{2} y_{2}^{2}-2 \varrho_{1}^{2} \varrho_{2}^{2} y_{1}^{2} x_{3}^{2}-2 \varrho_{1}^{2} \varrho_{2}^{2} x_{3}^{2} x_{2}^{2}-2 \varrho_{1}^{2} \varrho_{2}^{2} x_{3}^{2} y_{2}^{2}+2 \varrho_{1}^{2} \varrho_{2}^{2} y_{3}^{2} x_{2}^{2}+2 \varrho_{1}^{2} \varrho_{2}^{2} y_{3}^{2} y_{2}^{2}+ \\
& 4 \varrho_{1}^{2} \varrho_{3}^{2} x_{2}^{3} x_{3}-4 \varrho_{1}^{4} x_{2} x_{3} y_{3}^{2}-4 \varrho_{1}^{4} x_{2} x_{3} y_{2}^{2}+4 \varrho_{1}^{2} \varrho_{3}^{2} x_{2} x_{1} y_{3}^{2}+4 \varrho_{1}^{2} x_{2} \varrho_{3}^{2} x_{1} y_{2}^{2}-8 \varrho_{1}^{2} x_{2} \varrho_{2}^{2} x_{1} x_{3}^{2}- \\
& 8 \varrho_{1}^{2} x_{2} \varrho_{2}^{2} x_{1} y_{3}^{2}+4 \varrho_{1}^{2} x_{2} \varrho_{2}^{2} x_{3} y_{3}^{2}+4 \varrho_{1}^{2} x_{2} x_{3} \varrho_{3}^{2} x_{1}^{2}+4 \varrho_{1}^{2} x_{2} x_{3} \varrho_{3}^{2} y_{1}^{2}+4 \varrho_{1}^{2} x_{2} \varrho_{3}^{2} x_{1} x_{3}^{2}+4 \varrho_{1}^{2} \varrho_{2}^{2} x_{1} x_{3} y_{3}^{2}+ \\
& 4 \varrho_{1}^{2} \varrho_{3}^{2} x_{2} x_{3} y_{2}^{2}+4 \varrho_{1}^{2} \varrho_{2}^{2} x_{1} x_{3} x_{2}^{2}+4 \varrho_{1}^{2} \varrho_{2}^{2} x_{1} x_{3} y_{2}^{2}-8 \varrho_{1}^{2} x_{3} \varrho_{3}^{2} x_{1} x_{2}^{2}-8 \varrho_{1}^{2} x_{3} \varrho_{3}^{2} x_{1} y_{2}^{2}+4 \varrho_{1}^{2} x_{2} x_{3} \varrho_{2}^{2} x_{1}^{2}+ \\
& 4 \varrho_{1}^{2} x_{2} x_{3} \varrho_{2}^{2} y_{1}^{2} \\
& a_{4}=2 \varrho_{3}^{2} x_{2}-2 \varrho_{1}^{2} x_{2}-2 \varrho_{2}^{2} x_{3}+2 \varrho_{1}^{2} x_{3}-2 \varrho_{3}^{2} x_{1}+2 \varrho_{2}^{2} x_{1} \\
& a_{5}=2 \varrho_{1}^{2} y_{3}+2 \varrho_{3}^{2} y_{2}-2 \varrho_{1}^{2} y_{2}+2 \varrho_{2}^{2} y_{1}-2 \varrho_{2}^{2} y_{3}-2 \varrho_{3}^{2} y_{1} \\
& a_{6}=-\varrho_{3}^{2} y_{2}^{2}-\varrho_{2}^{2} x_{1}^{2}-\varrho_{2}^{2} y_{1}^{2}+\varrho_{1}^{2} x_{2}^{2}+\varrho_{1}^{2} y_{2}^{2}+\varrho_{2}^{2} x_{3}^{2}-\varrho_{1}^{2} x_{3}^{2}+\varrho_{2}^{2} y_{3}^{2}-\varrho_{3}^{2} x_{2}^{2}+\varrho_{3}^{2} y_{1}^{2}+\varrho_{3}^{2} x_{1}^{2}-\varrho_{1}^{2} y_{3}^{2}
\end{aligned}
$$

Wie bei der Linienkonfiguration mit zwei Sensoren treten beim Lösen von (3.21) auch bei der Ebenenkonfiguration mit drei Sensoren zwei (eine wahre und eine scheinbare) Lösungen auf

$$
\begin{aligned}
& y_{0, I, I I}=-\frac{a_{2}}{2 a_{1}} \pm \sqrt{\left(\frac{a_{2}}{2 a_{1}}\right)^{2}-\frac{a_{3}}{a_{1}}} \\
& x_{0, I, I I}=\frac{-a_{5} \cdot y_{0, I, I I}-a_{6}}{a_{4}} .
\end{aligned}
$$

Der Skalierungsfaktor $\gamma$ lässt sich über die Beziehung

$$
\gamma_{I, I I}=\frac{\varrho_{i}}{\left\|\mathbf{x}_{i}-\mathbf{x}_{0, I, I I}\right\|_{2}} \quad \text { mit } \quad \mathbf{x}_{0, I, I I}=\left(x_{0, I, I I}, y_{0, I, I I}\right)^{T}
$$

bestimmen, die für jeden Sensor $i$ gilt. 


\section{Lösung mittels Schnittkreisen}

Neben der Lösung mit Hilfe der Gröbner-Basen lässt sich auch ein geometrisch interpretierbarer Lösungsweg finden, der zudem Aussagen zur Existenz von Lösungen im gestörten Fall auf einfache Weise erlaubt (vgl. Abschnitt 3.3.4). Die hier vorgestellte Idee besteht darin, zunächst die Menge möglicher Quellorte für jeweils ein Paar von Sensoren zu bestimmen. Anschließend werden mehrere solche Mengen zum Schnitt gebracht, um den Quellort zu ermitteln.

Für die Sensorpaare gelten die Gleichungen (3.19). Bei drei Sensoren müssen damit also nur zwei Sensorpaare (hier 1,2 sowie 1,3) betrachtet werden. Wegen der Konsistenz von (3.18) im hier betrachteten ungestörten Fall liefert das mögliche dritte Sensorpaar 2,3 keine weitere Information (Gleichung).

Für ein festes $\gamma$ bildet die Menge aller Punkte, auf der sich aus der Sicht eines einzelnen Sensors $i$ die Quelle befinden kann, einen Kreis um den Sensorstandort (vgl. (3.18))

$$
\frac{\varrho_{i}}{\gamma}=\left\|\mathbf{x}_{0}-\mathbf{x}_{i}\right\|_{2}
$$

Die Menge aller Punkte, auf der sich aus der Sicht eines Sensorpaars die Quelle befinden kann, setzt sich damit aus den Schnittpunkten dieser Kreise für alle $\gamma$ zusammen.

Beispiel 3.2 Für den Fall einer Ebenenkonfiguration mit Sensoren in $\mathbf{x}_{1}=(1 \mathrm{~m}, 1 \mathrm{~m})^{T}$, $\mathbf{x}_{2}=(4.5 \mathrm{~m}, 1.5 \mathrm{~m})^{T}, \mathbf{x}_{3}=(4 \mathrm{~m}, 4 \mathrm{~m})^{T}$ und der Quelle bei $\mathbf{x}_{0, I}=(3 \mathrm{~m}, 3 \mathrm{~m})^{T}$ wird zunächst für das Sensorpaar 1, 2 die Menge möglicher Quellorte ermittelt. Dafür wird, wie in Abb. 3.7 (links) gezeigt, $\gamma$ im Bereich zwischen 1 und 5 variiert. Für jedes $\gamma$ entsteht für jeden Sensor ein Kreis um den Sensorstandort (Sensor 1: gepunktete Kreise, Sensor 2: gestrichelte Kreise). Die Schnittpunkte beider Kreise sind mögliche Quellorte aus der Sicht des Sensorpaars 1,2. Werden diese Schnittpunkte über alle $\gamma$ aufgetragen, entsteht wiederum ein Kreis, der so genannte Schnittkreis (durchgängiger Kreis). Ein Schnittkreis lässt sich auf gleiche Weise für das Sensorpaar 1,3 generieren, Abb. 3.7 (rechts).

Abbildung 3.8 zeigt die Schnittkreise für die Sensorpaare 1,2 und 1,3 mit den Mittelpunkten $\left(x_{S, 12}, y_{S, 12}\right)$ und $\left(x_{S, 13}, y_{S, 13}\right)$, die in Beispiel 3.2 entstehen. Wie erwartet, schneiden sich die Schnittkreise sowohl im wahren Quellort $\mathbf{x}_{0, I}=(3 \mathrm{~m}, 3 \mathrm{~m})^{T}$ als auch im scheinbaren Quellort $\mathbf{x}_{0, I I} \approx(6.2 \mathrm{~m}, 7.5 \mathrm{~m})^{T}$. Nachfolgend werden die Kreisgleichungen für die Schnittkreise analytisch hergeleitet. 

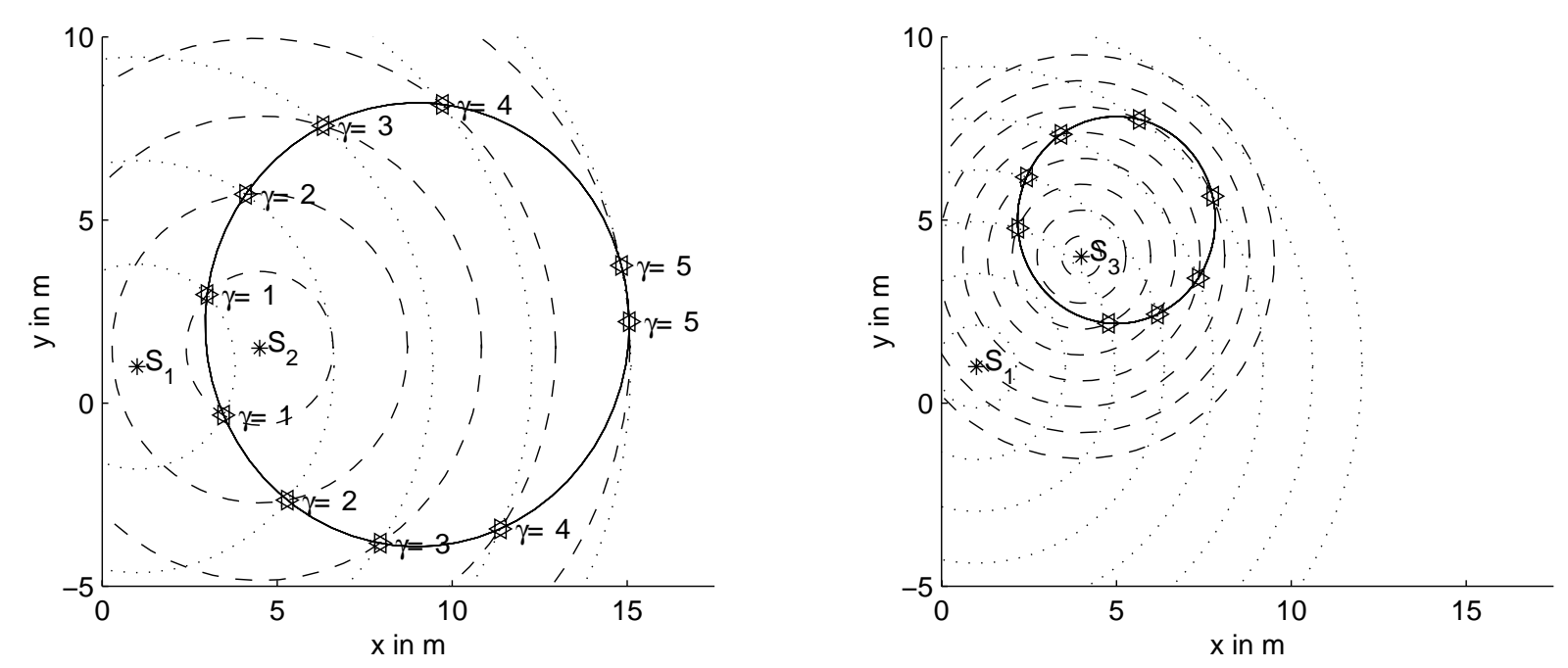

Abb. 3.7: Konstruktion des Schnittkreises durch Variieren von $\gamma$ für Sensorpaar 1,2 (links) und Sensorpaar 1,3 (rechts).

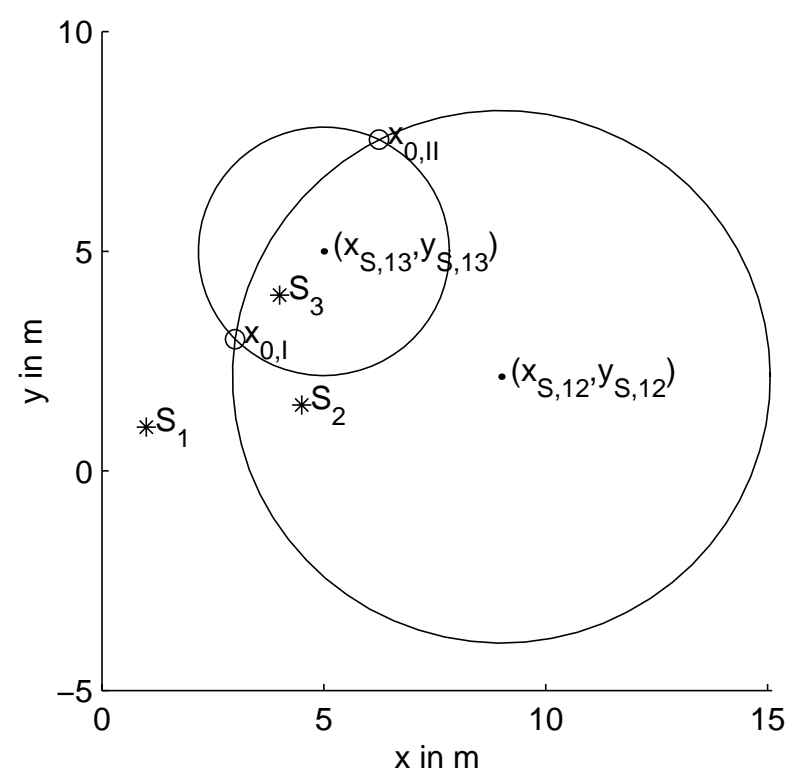

Abb. 3.8: Schnittkreise für Sensoren 1 und 2 bzw. 1 und 3 sowie wahrer und scheinbarer Quellort als Schnittpunkte der Schnittkreise.

Für die Menge der Punkte, auf der sich aus der Sicht des Sensorpaars 1,2 bzw. 1, 3 die Quelle befinden kann, gilt (vgl. (3.19))

$$
\begin{aligned}
& \varrho_{2}^{2} \cdot\left\|\mathbf{x}_{1}-\mathbf{x}_{0}\right\|_{2}^{2}=\varrho_{1}^{2} \cdot\left\|\mathbf{x}_{2}-\mathbf{x}_{0}\right\|_{2}^{2} \\
& \varrho_{3}^{2} \cdot\left\|\mathbf{x}_{1}-\mathbf{x}_{0}\right\|_{2}^{2}=\varrho_{1}^{2} \cdot\left\|\mathbf{x}_{3}-\mathbf{x}_{0}\right\|_{2}^{2}
\end{aligned}
$$

Ausmultiplizieren und Zusammenfassen dieser quadratischen Gleichungen in $x_{0}$ und $y_{0}$ 
liefert die Schnittkreisgleichungen

$$
\begin{aligned}
& \left(x_{0}-x_{S, 12}\right)^{2}+\left(y_{0}-y_{S, 12}\right)^{2}=r_{S, 12}^{2} \\
& \left(x_{0}-x_{S, 13}\right)^{2}+\left(y_{0}-y_{S, 13}\right)^{2}=r_{S, 13}^{2}
\end{aligned}
$$

mit den Mittelpunktskoordinaten

$$
x_{S, 12}=\frac{x_{1} \varrho_{2}^{2}-x_{2} \varrho_{1}^{2}}{\varrho_{2}^{2}-\varrho_{1}^{2}}, \quad y_{S, 12}=\frac{y_{1} \varrho_{2}^{2}-y_{2} \varrho_{1}^{2}}{\varrho_{2}^{2}-\varrho_{1}^{2}}
$$

bzw.

$$
x_{S, 13}=\frac{x_{1} \varrho_{3}^{2}-x_{3} \varrho_{1}^{2}}{\varrho_{3}^{2}-\varrho_{1}^{2}}, \quad y_{S, 13}=\frac{y_{1} \varrho_{3}^{2}-y_{3} \varrho_{1}^{2}}{\varrho_{3}^{2}-\varrho_{1}^{2}}
$$

und den Radien

$$
r_{S, 12}^{2}=\left(\frac{\varrho_{2}^{2} \mathbf{x}_{1}^{T}-\varrho_{1}^{2} \mathbf{x}_{2}^{T}}{\varrho_{2}^{2}-\varrho_{1}^{2}}\right)^{2}-\frac{\varrho_{2}^{2} \mathbf{x}_{1}^{T} \mathbf{x}_{1}-\varrho_{1}^{2} \mathbf{x}_{2}^{T} \mathbf{x}_{2}}{\varrho_{2}^{2}-\varrho_{1}^{2}}
$$

bzw.

$$
r_{S, 13}^{2}=\left(\frac{\varrho_{2}^{2} \mathbf{x}_{1}^{T}-\varrho_{1}^{2} \mathbf{x}_{3}^{T}}{\varrho_{2}^{2}-\varrho_{1}^{2}}\right)^{2}-\frac{\varrho_{2}^{2} \mathbf{x}_{1}^{T} \mathbf{x}_{1}-\varrho_{1}^{2} \mathbf{x}_{3}^{T} \mathbf{x}_{3}}{\varrho_{2}^{2}-\varrho_{1}^{2}} .
$$

Die Schnittpunkte dieser zwei Schnittkreise stellen die gesuchte Lösung für den Quellort $\left(x_{0}, y_{0}\right)$ dar. Im hier betrachteten ungestörten Fall existiert immer mindestens ein Schnittpunkt der Schnittkreise, nämlich der wahre Quellort. Im Allgemeinen besitzen zwei Schnittkreise jedoch zwei Schnittpunkte mit den Koordinaten

$$
\begin{aligned}
& y_{0, I / I I}= 1 / 2 \cdot\left(y_{S, 13}^{3}-y_{S, 13} \cdot r_{S, 13}^{2}-y_{S, 12} \cdot r_{S, 12}^{2}-2 \cdot x_{S, 13} \cdot x_{S, 12} \cdot y_{S, 13}+y_{S, 12}^{3}+x_{S, 12}^{2} \cdot y_{S, 12}+x_{S, 12}^{2} \cdot y_{S, 13}+\right. \\
& y_{S, 12} \cdot r_{S, 13}^{2}+x_{S, 13}^{2} \cdot y_{S, 13}+r_{S, 12}^{2} \cdot y_{S, 13}-y_{S, 12}^{2} \cdot y_{S, 13}-y_{S, 12} \cdot y_{S, 13}^{2}-2 \cdot x_{S, 13} \cdot x_{S, 12} \cdot y_{S, 12}+y_{S, 12} \cdot \\
& x_{S, 13}^{2} \quad \pm \quad\left(-\left(4 \cdot x_{S, 13} \cdot x_{S, 12} \cdot r_{S, 13}^{2}-4 \cdot x_{S, 13} \cdot x_{S, 12} \cdot y_{S, 13}^{2}+4 \cdot x_{S, 13} \cdot x_{S, 12} \cdot r_{S, 12}^{2}-4 \cdot x_{S, 13} \cdot\right.\right. \\
& x_{S, 12} \cdot y_{S, 12}^{2}-4 \cdot x_{S, 12}^{2} \cdot y_{S, 13} \cdot y_{S, 12}+8 \cdot x_{S, 13} \cdot x_{S, 12} \cdot y_{S, 13} \cdot y_{S, 12}-4 \cdot x_{S, 13} \cdot x_{S, 12}^{3}-4 \cdot x_{S, 13}^{3} \cdot x_{S, 12}- \\
& 2 \cdot y_{S, 12}^{2} \cdot r_{S, 13}^{2}-2 \cdot r_{S, 12}^{2} \cdot x_{S, 13}^{2}-2 \cdot r_{S, 12}^{2} \cdot y_{S, 13}^{2}-2 \cdot r_{S, 12}^{2} \cdot r_{S, 13}^{2}+2 \cdot x_{S, 13}^{2} \cdot y_{S, 13}^{2}-2 \cdot x_{S, 13}^{2} \cdot r_{S, 13}^{2}- \\
& 2 \cdot y_{S, 13}^{2} \cdot r_{S, 13}^{2}+x_{S, 12}^{4}+y_{S, 12}^{4}+r_{S, 12}^{4}+x_{S, 13}^{4}+y_{S, 13}^{4}+r_{S, 13}^{4}+2 \cdot x_{S, 12}^{2} \cdot y_{S, 12}^{2}-2 \cdot x_{S, 12}^{2} \cdot r_{S, 12}^{2}+6 \cdot \\
& x_{S, 12}^{2} \cdot x_{S, 13}^{2}+2 \cdot x_{S, 12}^{2} \cdot y_{S, 13}^{2}-2 \cdot x_{S, 12}^{2} \cdot r_{S, 13}^{2}-2 \cdot y_{S, 12}^{2} \cdot r_{S, 12}^{2}+2 \cdot y_{S, 12}^{2} \cdot x_{S, 13}^{2}+6 \cdot y_{S, 12}^{2} \cdot y_{S, 13}^{2}- \\
& 4 \cdot y_{S, 12}^{3} \cdot y_{S, 13}-4 \cdot x_{S, 13}^{2} \cdot y_{S, 13} \cdot y_{S, 12}+4 \cdot y_{S, 12} \cdot r_{S, 12}^{2} \cdot y_{S, 13}+4 \cdot y_{S, 13} \cdot r_{S, 13}^{2} \cdot y_{S, 12}-4 \cdot y_{S, 13}^{3} \cdot \\
&\left.\left.\left.y_{S, 12}\right) \cdot\left(x_{S, 12}-x_{S, 13}\right)^{2}\right)^{(1 / 2)}\right) /\left(-2 \cdot x_{S, 13} \cdot x_{S, 12}-2 \cdot y_{S, 12} \cdot y_{S, 13}+x_{S, 13}^{2}+y_{S, 13}^{2}+x_{S, 12}^{2}+y_{S, 12}^{2}\right) \\
& \\
& x_{0, I / I I}=-2 \cdot\left(y_{S, 13}-y_{S, 12}\right) \cdot y_{0, I / I I}+\left(x_{S, 13}^{2}-x_{S, 12}^{2}\right)+\left(y_{S, 13}^{2}-y_{S, 12}^{2}\right)+\left(r_{S, 12}^{2}-r_{S, 13}^{2}\right) \\
& 2 \cdot\left(x_{S, 13}-x_{S, 12}\right)
\end{aligned}
$$

Der Spezialfall eines Berührungspunktes der beiden Schnittkreise und damit nur einer Lösung für den Quellort wird durch (3.31) automatisch berücksichtigt (der Term in der Lösung für $y_{0}$ hinter \pm wird zu null; und es gilt $\left.y_{0, I}=y_{0, I I}\right)$. 


\subsubsection{Raumkonfiguration}

Bei der Raumkonfiguration können die Sensoren und die Quelle beliebig im Raum liegen. Zum Bestimmen der unbekannten Parameter $\mathbf{x}_{0}=\left(x_{0}, y_{0}, z_{0}\right)^{T}$ und $\gamma$ sind mindestens vier Sensoren notwendig. Es muss also das Gleichungssystem (3.18) für $i=1, \ldots, 4$ gelöst werden.

Dazu lässt sich prinzipiell - wie auch bei der Ebenenkonfiguration - eine Gröbner-Basis verwenden. Für die Raumkonfiguration vermochte es die verwendete MAPLE-Version aus Kapazitätsgründen nicht, eine Gröbner-Basis zu finden. Auch eine geometrisch interpretierbare Lösung über Schnittkurven erweist sich wegen der zu lösenden Kugelschnittprobleme als ungeeignet. Aus diesem Grund wird hier ein alternativer Weg erarbeitet, der sich ebenso für die Linien- und Ebenenkonfiguration nutzen lässt.

Zunächst wird das nichtlineare Gleichungssystem (3.18) für $i=1, \ldots, 4$ mit vier unbekannten Parametern in das unterbestimmte, lineare Gleichungssystem mit fünf Parametern

$$
\mathbf{A} \cdot \boldsymbol{\theta}=\mathbf{b}
$$

mit

$$
\mathbf{A}=\left(\begin{array}{ccc}
2 \mathbf{x}_{1}^{T} & \varrho_{1}^{2} & -1 \\
2 \mathbf{x}_{2}^{T} & \varrho_{2}^{2} & -1 \\
2 \mathbf{x}_{3}^{T} & \varrho_{3}^{2} & -1 \\
2 \mathbf{x}_{4}^{T} & \varrho_{4}^{2} & -1
\end{array}\right), \quad \boldsymbol{\theta}=\left(\begin{array}{c}
\mathbf{x}_{0} \\
\frac{1}{\gamma^{2}} \\
\mathbf{x}_{0}^{T} \mathbf{x}_{0}
\end{array}\right), \quad b=\left(\begin{array}{c}
\mathbf{x}_{1}^{T} \mathbf{x}_{1} \\
\mathbf{x}_{2}^{T} \mathbf{x}_{2} \\
\mathbf{x}_{3}^{T} \mathbf{x}_{3} \\
\mathbf{x}_{4}^{T} \mathbf{x}_{4}
\end{array}\right)
$$

überführt. Dabei ist zu beachten, dass der fünfte Parameter $\mathbf{x}_{0}^{T} \mathbf{x}_{0}$ direkt von den ersten drei Parametern, die $\mathbf{x}_{0}$ bilden, abhängt. Das führt auf eine Restriktion. Mit der verkürzten Schreibweise $\theta_{i}$ für das $i$-te Element bzw. $\boldsymbol{\theta}_{i: j}$ für die Elemente $i$ bis $j$ von $\boldsymbol{\theta}$ lautet die Restriktion

$$
\theta_{5}=\boldsymbol{\theta}_{1: 3}^{T} \boldsymbol{\theta}_{1: 3}
$$

Die allgemeine Lösung für (3.32) setzt sich aus der Lösung des homogenen Gleichungssystems $\boldsymbol{\theta}_{h}$ und einer partikulären Lösung des inhomogenen Systems $\boldsymbol{\theta}_{p}$ zusammen [10, S. 3], [90, S.24]

$$
\boldsymbol{\theta}=\boldsymbol{\theta}_{p}+\lambda \cdot \boldsymbol{\theta}_{h}
$$

mit

$$
\boldsymbol{\theta}_{p}=\mathbf{A}^{+} \cdot \mathbf{b}, \quad \boldsymbol{\theta}_{h}=\left(\mathbf{I}_{5}-\mathbf{A}^{+} \mathbf{A}\right) \cdot \mathbf{g} .
$$

Dabei bezeichnet $\mathbf{A}^{+}$die Moore-Penrose-Inverse, $\mathbf{I}_{5}$ ist die Einheitsmatrix und $\mathbf{g}$ ein beliebiger Vektor. 
Der Ausdruck $\left(\mathbf{I}_{5}-\mathbf{A}^{+} \mathbf{A}\right)$ bildet jeden beliebigen Vektor $\mathbf{g}$ in den Nullraum $N(\mathbf{A})$ der Matrix A ab. Die Dimension des Nullraums ist gleich dem Defekt (5- $\operatorname{Rg}(\mathbf{A})$ ) [90, S.24]. Wenn alle Sensoren verschiedene Standorte $\mathbf{x}_{i}$ besitzen, gilt $\operatorname{Rg}(\mathbf{A})=4 . N(\mathbf{A})$ ist also eindimensional. Deshalb reicht es aus, nur eine Spalte $j$, von $\left(\mathbf{I}_{5}-\mathbf{A}^{+} \mathbf{A}\right)$, die mindestens ein Element verschieden von null besitzt, zu betrachten

$$
\boldsymbol{\theta}_{h}=\left(\mathbf{I}_{5}-\mathbf{A}^{+} \mathbf{A}\right) \cdot \mathbf{e}_{j}^{T}
$$

Um $\lambda$ zu bestimmen, wird die Restriktion (3.34) genutzt. Aus (3.35) folgt

$$
\theta_{p, 5}+\lambda \cdot \theta_{h, 5}=\left(\boldsymbol{\theta}_{p, 1: 3}+\lambda \cdot \boldsymbol{\theta}_{h, 1: 3}\right)^{T} \cdot\left(\boldsymbol{\theta}_{p, 1: 3}+\lambda \cdot \boldsymbol{\theta}_{h, 1: 3}\right)
$$

Diese quadratische Gleichung in $\lambda$

$$
\boldsymbol{\theta}_{h, 1: 3}^{T} \cdot \boldsymbol{\theta}_{h, 1: 3} \cdot \lambda^{2}+\left(2 \cdot \boldsymbol{\theta}_{p, 1: 3}^{T} \cdot \boldsymbol{\theta}_{h, 1: 3}-\theta_{h, 5}\right) \cdot \lambda+\boldsymbol{\theta}_{p, 1: 3}^{T} \cdot \boldsymbol{\theta}_{p, 1: 3}-\theta_{p, 5}=0
$$

besitzt die Lösungen

$$
\lambda_{I, I I}=-\frac{2 \cdot \boldsymbol{\theta}_{p, 1: 3}^{T} \cdot \boldsymbol{\theta}_{h, 1: 3}-\theta_{h, 5}}{2 \cdot \boldsymbol{\theta}_{h, 1: 3}^{T} \cdot \boldsymbol{\theta}_{h, 1: 3}} \pm \sqrt{\left(\frac{2 \cdot \boldsymbol{\theta}_{p, 1: 3}^{T} \cdot \boldsymbol{\theta}_{h, 1: 3}-\theta_{h, 5}}{2 \cdot \boldsymbol{\theta}_{h, 1: 3}^{T} \cdot \boldsymbol{\theta}_{h, 1: 3}}\right)^{2}-\frac{\boldsymbol{\theta}_{p, 1: 3}^{T} \cdot \boldsymbol{\theta}_{p, 1: 3}+\theta_{p, 5}}{\boldsymbol{\theta}_{h, 1: 3}^{T} \cdot \boldsymbol{\theta}_{h, 1: 3}}} .
$$

Mit diesen beiden Lösungen für $\lambda$ werden mit (3.35) erneut zwei verschiedene Lösungen (die wahre und eine scheinbare) für das Quellenlokalisierungsproblem erhalten

$$
\boldsymbol{\theta}_{I, I I}=\boldsymbol{\theta}_{p}+\lambda_{I, I I} \cdot \boldsymbol{\theta}_{h}
$$

Da im ungestörten Fall stets eine Lösung für den Quellort existiert, muss der Radikant in (3.40) größer oder gleich null sein. Ist er genau null, dann existiert nur eine Lösung für den Quellort; wahrer und scheinbarer Quellort sind dann identisch.

\subsubsection{Halbraumkonfiguration}

Bei der Halbraumkonfiguration mit der Quelle auf der impermeablen Oberfläche $\left(z_{0}=0\right)$ sind ebenfalls wieder die unbekannten Parameter $\mathbf{x}_{0}=\left(x_{0}, y_{0}\right)^{T}$ und $\gamma$ zu bestimmen. Dazu sind mindestens drei Sensoren notwendig. Analog zur Raumkonfiguration wird das nichtlineare Gleichungssystem (3.18) mit drei Parametern in ein lineares Gleichungssystem mit vier Parametern überführt

$$
\mathbf{A}=\left(\begin{array}{cccc}
2 x_{1} & 2 y_{1} & \varrho_{1}^{2} & -1 \\
2 x_{2} & 2 y_{2} & \varrho_{2}^{2} & -1 \\
2 x_{3} & 2 y_{3} & \varrho_{3}^{2} & -1
\end{array}\right), \quad \boldsymbol{\theta}=\left(\begin{array}{c}
\mathbf{x}_{0} \\
\frac{1}{\gamma^{2}} \\
\mathbf{x}_{0}^{T} \mathbf{x}_{0}
\end{array}\right), \quad \mathbf{b}=\left(\begin{array}{c}
\mathbf{x}_{1}^{T} \mathbf{x}_{1} \\
\mathbf{x}_{2}^{T} \mathbf{x}_{2} \\
\mathbf{x}_{3}^{T} \mathbf{x}_{3}
\end{array}\right)
$$


Die Lösung dieses Gleichungssystems erfolgt auf die gleiche Weise wie beim Fall des unendlichen Mediums. (3.40) wird damit zu

$$
\lambda_{I, I I}=-\frac{2 \cdot \boldsymbol{\theta}_{p, 1: 2}^{T} \cdot \boldsymbol{\theta}_{h, 1: 2}-\theta_{h, 4}}{2 \cdot \boldsymbol{\theta}_{h, 1: 2}^{T} \cdot \boldsymbol{\theta}_{h, 1: 2}} \pm \sqrt{\left(\frac{2 \cdot \boldsymbol{\theta}_{p, 1: 2}^{T} \cdot \boldsymbol{\theta}_{h, 1: 2}-\theta_{h, 4}}{2 \cdot \boldsymbol{\theta}_{h, 1: 2}^{T} \cdot \boldsymbol{\theta}_{h, 1: 2}}\right)^{2}-\frac{\boldsymbol{\theta}_{p, 1: 2}^{T} \cdot \boldsymbol{\theta}_{p, 1: 2}+\theta_{p, 4}}{\boldsymbol{\theta}_{h, 1: 2}^{T} \cdot \boldsymbol{\theta}_{h, 1: 2}}}
$$

\subsubsection{Allgemeine Lösung}

Das für die Raum- bzw. Halbraumkonfiguration vorgestellte Verfahren zum Lösen von (3.18) auf der Basis minimaler Sensoranzahl $m$ lässt sich auch für die Linien- und Ebenenkonfiguration anwenden. Die Basis der Lösung bildet in allen Fällen das lineare Gleichungssystem

$$
\mathbf{A} \cdot \boldsymbol{\theta}=\mathbf{b} \quad \text { mit der Restriktion } \quad \theta_{n+2}=\boldsymbol{\theta}_{1: n}^{T} \boldsymbol{\theta}_{1: n} .
$$

Dabei bezeichnet $n$ fortan die Dimension des Quellorts $\mathbf{x}_{0}$. Die Größen $\mathbf{A}, \boldsymbol{\theta}, \mathbf{b}$ für die einzelnen Fälle ergeben sich in Abhängigkeit von der minimalen Sensoranzahl $m$ aus

$$
\mathbf{A}=\left(\begin{array}{ccc}
2 \mathbf{x}_{1}^{T} & \varrho_{1}^{2} & -1 \\
\vdots & \vdots & \vdots \\
2 \mathbf{x}_{m}^{T} & \varrho_{m}^{2} & -1
\end{array}\right), \quad \boldsymbol{\theta}=\left(\begin{array}{c}
\mathbf{x}_{0} \\
\frac{1}{\gamma^{2}} \\
\mathbf{x}_{0}^{T} \mathbf{x}_{0}
\end{array}\right), \quad \mathbf{b}=\left(\begin{array}{c}
\mathbf{x}_{1}^{T} \mathbf{x}_{1} \\
\vdots \\
\mathbf{x}_{m}^{T} \mathbf{x}_{m}
\end{array}\right)
$$

Das Gleichungssystem besitzt die Lösungen

$$
\boldsymbol{\theta}_{I, I I}=\boldsymbol{\theta}_{p}+\lambda_{I, I I} \cdot \boldsymbol{\theta}_{h}
$$

mit

$$
\boldsymbol{\theta}_{p}=\mathbf{A}^{+} \cdot \mathbf{b}, \quad \boldsymbol{\theta}_{h}=\left(\mathbf{I}_{n+2}-\mathbf{A}^{+} \mathbf{A}\right) \cdot \mathbf{e}_{j}^{T}
$$

Die Lösungen für $\lambda$ lauten

$$
\begin{aligned}
\lambda_{I, I I}= & -\frac{2 \cdot \boldsymbol{\theta}_{p, 1: n}^{T} \cdot \boldsymbol{\theta}_{h, 1: n}-\theta_{h, n+2}}{2 \cdot \boldsymbol{\theta}_{h, 1: n}^{T} \cdot \boldsymbol{\theta}_{h, 1: n}} \\
& \pm \sqrt{\left(\frac{2 \cdot \boldsymbol{\theta}_{p, 1: n}^{T} \cdot \boldsymbol{\theta}_{h, 1: n}-\theta_{h, n+2}}{2 \cdot \boldsymbol{\theta}_{h, 1: n}^{T} \cdot \boldsymbol{\theta}_{h, 1: n}}\right)^{2}-\frac{\boldsymbol{\theta}_{p, 1: n}^{T} \cdot \boldsymbol{\theta}_{p, 1: n}+\theta_{p, n+2}}{\boldsymbol{\theta}_{h, 1: n}^{T} \cdot \boldsymbol{\theta}_{h, 1: n}}}
\end{aligned}
$$




\subsection{Untersuchung der Lösung}

\subsubsection{Eigenschaften von wahrem und scheinbarem Quellort}

Bei der Ermittlung des Quellorts mittels skalierbarer Sensor-Quell-Abstände werden mit minimaler Sensoranzahl eine wahre und eine scheinbare Lösung erhalten. Nachfolgend wird die Lage dieser beiden Lösungen für die Ebenenkonfiguration näher untersucht. Anschließend werden die dabei gewonnenen Erkenntnisse auf den Fall der Raum- bzw. Halbraumkonfiguration erweitert.

\section{Ebenenkonfiguration}

Im Beispiel 3.2 wurden für den Quellort die Lösungen $\mathbf{x}_{0, I}=(3 \mathrm{~m}, 3 \mathrm{~m})^{T}$ und $\mathbf{x}_{0, I I} \approx$ $(6.2 \mathrm{~m}, 7.5 \mathrm{~m})^{T}$ erhalten. In Abb. 3.9 sind die stationären Konzentrationsprofile für beide Quellorte und die zugehörigen Quellintensitäten $q_{0, I}=1 \mathrm{mg} / \mathrm{s}$ und $q_{0, I I}=2.96 \mathrm{mg} / \mathrm{s}$ über der $(x, y)$-Ebene aufgetragen.

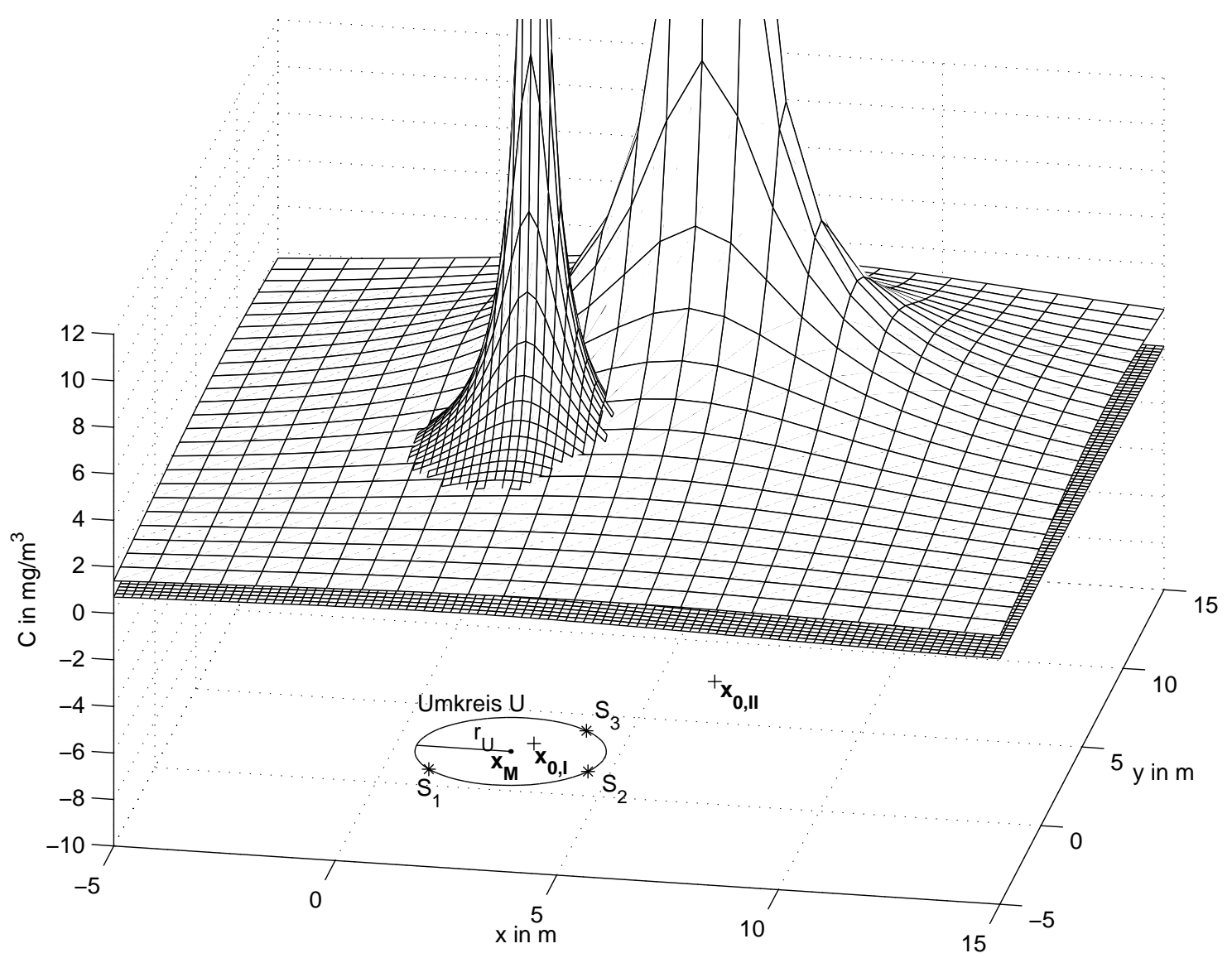

Abb. 3.9: Gleiche Konzentrationen im Umkreis der 3 Sensoren bei wahrem und scheinbarem Quellort. 
Es ist zu erkennen, dass die beiden möglichen Quellorte nicht nur an den drei Sensorstandorten die gleichen stationären Konzentrationen aufweisen, sondern dass eine Schnittkurve der Konzentrationsprofile existiert. Wird diese Schnittkurve in die $(x, y)$-Ebene projiziert, so entsteht genau der Kreis, auf dem alle Sensoren liegen (infolge der 3d-Darstellung erscheint der Kreis in Abb. 3.9 verzerrt). Dieser Kreis wird als Umkreis der Sensoren $U$ (Mittelpunkt $\mathbf{x}_{M}$, Radius $r_{U}$ ) bezeichnet.

Bei der Untersuchung der beiden möglichen Quellorte entsteht die Vermutung, dass der wahre und der scheinbare Quellort am Umkreis $U$ gespiegelte Punkte ${ }^{4}$ sind. Ist der Punkt $\mathbf{x}_{0, I}$ ein möglicher Quellort, dann liegt der zweite mögliche Quellort $\mathbf{x}_{0, I I}$ auf dem Strahl $\mathbf{x}_{M} \mathbf{x}_{0, I}$ und es gilt (vgl. Abb. 3.10)

$$
\left\|\mathbf{x}_{0, I}-\mathbf{x}_{M}\right\|_{2} \cdot\left\|\mathbf{x}_{0, I I}-\mathbf{x}_{M}\right\|_{2}=r_{U}^{2}
$$

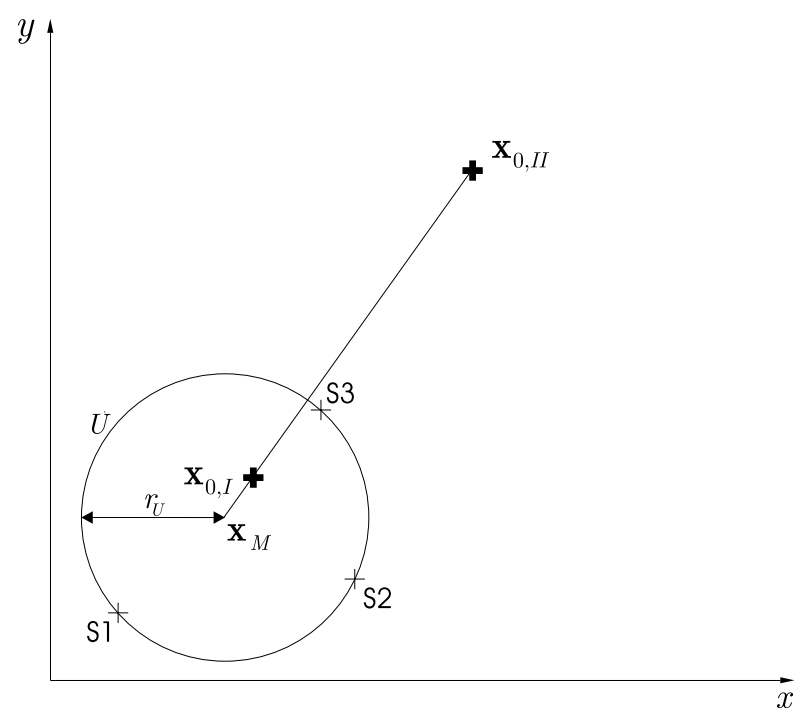

Abb. 3.10: Lösungen als gespiegelte Punkte am Umkreis $U$ der Sensoren.

Um diese Behauptung zu beweisen, muss gezeigt werden, dass

- die Gerade $\overline{\mathbf{x}_{0, I} \mathbf{x}_{0, I I}}$ den Mittelpunkt $\mathbf{x}_{M}$ schneidet und

- die Beziehung (3.49) gilt.

Der Nachweis wird anhand der allgemeinen Lösung über (3.44) und (3.45) geführt. Für die Ebenenkonfiguration folgt daraus

$$
\mathbf{A} \cdot \boldsymbol{\theta}=\mathbf{b}
$$

\footnotetext{
${ }^{4}$ Ist der Punkt $\mathbf{x}^{\prime}$ der am Kreis $K$ (Mittelpunkt $\mathbf{x}_{K}$, Radius $r_{K}$ ) gespiegelte Punkt zu $\mathbf{x}$, dann befindet er sich auf dem Strahl $\mathbf{x}_{K} \mathbf{x}$ und es gilt $\left\|\mathbf{x}-\mathbf{x}_{k}\right\|_{2} \cdot\left\|\mathbf{x}^{\prime}-\mathbf{x}_{K}\right\|_{2}=r_{K}^{2}[99,102]$.
} 
mit

$$
\mathbf{A}=\left(\begin{array}{ccc}
2 \mathbf{x}_{1}^{T} & \varrho_{1}^{2} & -1 \\
2 \mathbf{x}_{2}^{T} & \varrho_{2}^{2} & -1 \\
2 \mathbf{x}_{3}^{T} & \varrho_{3}^{2} & -1
\end{array}\right), \quad \boldsymbol{\theta}=\left(\begin{array}{c}
\mathbf{x}_{0} \\
\frac{1}{\gamma^{2}} \\
\mathbf{x}_{0}^{T} \mathbf{x}_{0}
\end{array}\right), \quad \mathbf{b}=\left(\begin{array}{c}
\mathbf{x}_{1}^{T} \mathbf{x}_{1} \\
\mathbf{x}_{2}^{T} \mathbf{x}_{2} \\
\mathbf{x}_{3}^{T} \mathbf{x}_{3}
\end{array}\right)
$$

Die beiden Lösungen entstehen mit der homogenen Lösung $\boldsymbol{\theta}_{h}$ und einer partikulären Lösung $\boldsymbol{\theta}_{p}$ sowie zwei Werten für $\lambda$

$$
\boldsymbol{\theta}_{I, I I}=\boldsymbol{\theta}_{p}+\lambda_{I, I I} \cdot \boldsymbol{\theta}_{h}
$$

Die Ortskoordinaten sind in den ersten beiden Komponenten von $\boldsymbol{\theta}$ enthalten.

Da $\lambda \cdot \boldsymbol{\theta}_{h}$ die Gerade durch $\overline{\mathbf{x}_{0, I} \mathbf{x}_{0, I I}}$ beschreibt, müssen alle partikulären Lösungen ebenfalls auf dieser Geraden liegen. Existiert eine partikuläre Lösung, die sich auf dem Mittelpunkt $\mathbf{x}_{M}$ von $U$ befindet, dann ist nachgewiesen, dass die Gerade $\overline{\mathbf{x}_{0, I} \mathbf{x}_{0, I I}}$ den Mittelpunkt $\mathbf{x}_{M}$ schneidet.

In den weiteren Betrachtungen erweist sich ein Koordinatensystem als vorteilhaft, das so gelegt wird, dass sein Ursprung genau im Mittelpunkt $\mathbf{x}_{M}$ des Umkreises der Sensoren liegt. In diesem Fall ist $\mathbf{x}_{M}=\mathbf{0}$.

Damit gilt

$$
\mathbf{x}_{1}^{T} \mathbf{x}_{1}=\mathbf{x}_{2}^{T} \mathbf{x}_{2}=\mathbf{x}_{3}^{T} \mathbf{x}_{3}=r_{U}^{2} .
$$

Das lineare Gleichungssystem (3.50) lässt sich mit (3.53) als homogenes Gleichungssystem

$$
\mathbf{A} \cdot \boldsymbol{\theta}=\mathbf{0}
$$

mit

$$
\mathbf{A}=\left(\begin{array}{ccc}
2 \mathbf{x}_{1}^{T} & \varrho_{1}^{2} & -1 \\
2 \mathbf{x}_{2}^{T} & \varrho_{2}^{2} & -1 \\
2 \mathbf{x}_{3}^{T} & \varrho_{3}^{2} & -1
\end{array}\right), \quad \boldsymbol{\theta}=\left(\begin{array}{c}
\mathbf{x}_{0} \\
\frac{1}{\gamma^{2}} \\
\mathbf{x}_{0}^{T} \mathbf{x}_{0}+r_{U}^{2}
\end{array}\right)
$$

schreiben. Eine partikuläre Lösung dieses Gleichungssystems ist die triviale Lösung

$$
\boldsymbol{\theta}_{p}=\mathbf{0}
$$

die sich auf dem Mittelpunkt $\mathbf{x}_{M}$ von $U$ befindet.

Mit $\mathbf{x}_{M}=\mathbf{0}$ lautet die Forderung (3.49)

$$
\left\|\mathbf{x}_{0, I}\right\|_{2} \cdot\left\|\mathbf{x}_{0, I I}\right\|_{2}=r_{U}^{2}
$$


Da $\boldsymbol{\theta}_{p}=\mathbf{0}$ gilt, ist wegen (3.52)

$$
\boldsymbol{\theta}_{I, I I}=\lambda_{I, I I} \cdot\left(\begin{array}{c}
\mathbf{x}_{0} \\
\frac{1}{\gamma^{2}} \\
\mathbf{x}_{0}^{T} \mathbf{x}_{0}+r_{U}^{2}
\end{array}\right)
$$

also

$$
\mathbf{x}_{0, I, I I}=\lambda_{I, I I} \cdot \boldsymbol{\theta}_{h, 1: 2}
$$

Insbesondere gilt

$$
\begin{aligned}
\left\|\mathbf{x}_{0, I}\right\|_{2} & =\lambda_{I} \cdot\left\|\boldsymbol{\theta}_{h, 1: 2}\right\|_{2} \\
\left\|\mathbf{x}_{0, I I}\right\|_{2} & =\lambda_{I I} \cdot\left\|\boldsymbol{\theta}_{h, 1: 2}\right\|_{2}
\end{aligned}
$$

Die Forderung (3.57) kann damit als

$$
\lambda_{I} \cdot \lambda_{I I}=\frac{r_{U}^{2}}{\boldsymbol{\theta}_{h, 1: 2}^{T} \boldsymbol{\theta}_{h, 1: 2}} .
$$

geschrieben werden.

$\lambda_{I}$ und $\lambda_{I I}$ ergeben sich hier mit der aus (3.54) und (3.55) resultierenden Restriktion

$$
\theta_{4}-r_{U}^{2}=\boldsymbol{\theta}_{1: 2}^{T} \boldsymbol{\theta}_{1: 2}
$$

Mit (3.35) wird daraus die quadratische Gleichung

$$
\lambda^{2}-\frac{\theta_{h, 4}}{\boldsymbol{\theta}_{h, 1: 2}^{T} \boldsymbol{\theta}_{h, 1: 2}} \cdot \lambda+\frac{r_{U}^{2}}{\boldsymbol{\theta}_{h, 1: 2}^{T} \boldsymbol{\theta}_{h, 1: 2}}=0
$$

zur Bestimmung von $\lambda_{I}$ und $\lambda_{I I}$ erhalten. Der Wurzelsatz von Vieta lässt erkennen, dass die Forderung (3.61) durch (3.63) erfüllt wird.

Damit wurde gezeigt, dass der wahre und der scheinbare Quellort am Umkreis der Sensoren $U$ gespiegelte Punkte sind. Interessant sind zwei Spezialfälle:

- Befindet sich der wahre Quellort auf $U$, dann sind wahrer und scheinbarer Quellort identisch. Die zugehörigen Schnittkreise berühren sich im Quellort (Abb. 3.11).

- Befindet sich der wahre Quellort im Mittelpunkt von $U$, dann liegt der scheinbare Quellort unendlich weit entfernt von $U$. Umgekehrt gilt: Liegt der wahre Quellort unendlich/sehr weit entfernt von $U$, dann befindet sich der scheinbare Quellort im Mittelpunkt von $U$. Die zugehörigen Schnittkreise entarten zu Geraden, die sich nur in einem Punkt, nämlich dem Mittelpunkt $\mathbf{x}_{M}$ von $U$, schneiden (Abb. 3.12). 


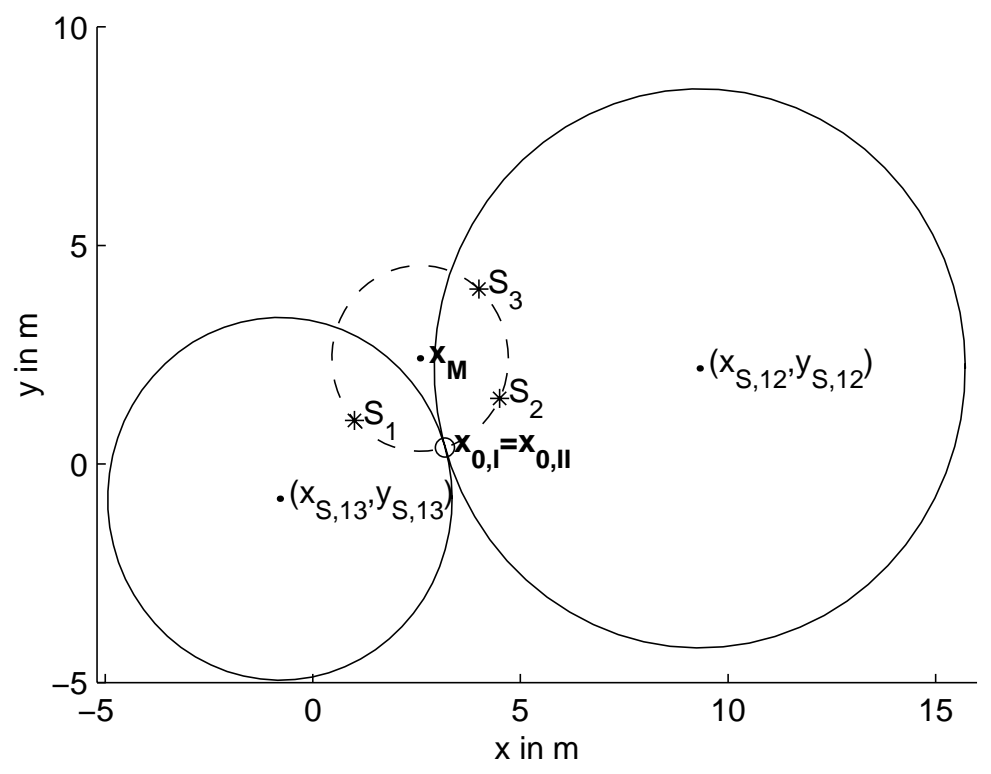

Abb. 3.11: Wahrer und scheinbarer Quellort befinden sich auf dem Umkreis $U$ der Sensoren und sind identisch. Die zugehörigen Schnittkreise berühren sich in diesem Punkt.

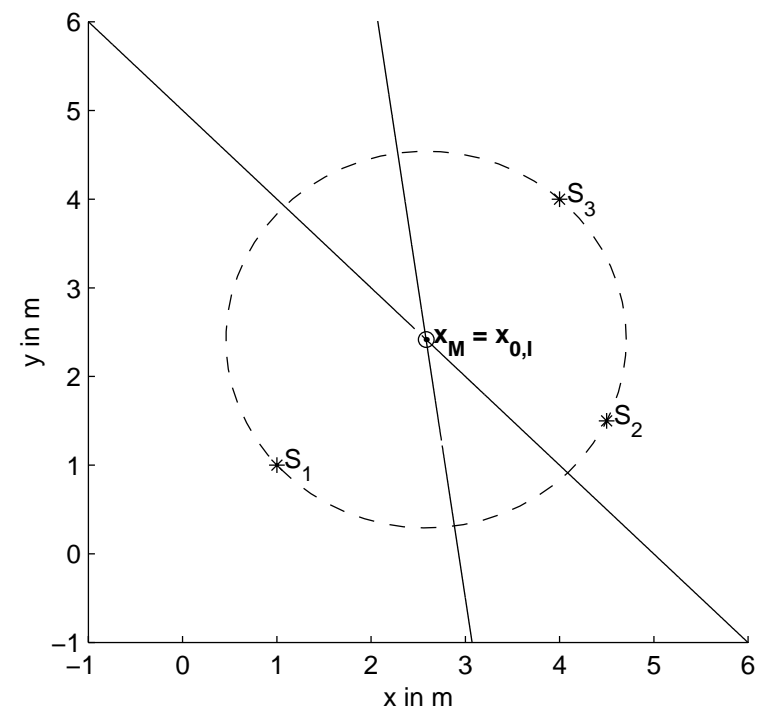

Abb. 3.12: Der wahre Quellort befindet sich im Mittelpunkt des Umkreises $U$ der Sensoren . Die Schnittkreise entarten zu Geraden und schneiden sich nur im Mittelpunkt $\mathbf{x}_{M}$ von $U$.

\section{Raumkonfiguration}

Bei der Untersuchung der Lage der beiden Lösungen für den Quellort bei Raumkonfiguration ist im Gegensatz zur Ebenenkonfiguration nicht der Umkreis $U$, sondern die Umkugel $U$ der dann notwendigen vier Sensoren zu betrachten. Werden analog zur Ebenenkonfiguration der Radius der Umkugel mit $r_{U}$ und deren Mittelpunkt mit $\mathbf{x}_{M}$ bezeichnet, so 
gelten alle gemachten Aussagen auch für den Fall einer Raumkonfiguration. Die beiden Lösungen sind also gespiegelte Punkte an der Umkugel $U$ und es gilt

$$
\left\|\mathbf{x}_{0, I}-\mathbf{x}_{M}\right\|_{2} \cdot\left\|\mathbf{x}_{0, I I}-\mathbf{x}_{M}\right\|_{2}=r_{U}^{2}
$$

Der Nachweis verläuft analog zum Fall der Ebenenkonfiguration, da er auf der allgemeinen Lösung (3.44) und (3.45) beruht.

\section{Halbraumkonfiguration}

Die Untersuchung der Lage der beiden möglichen Quellorte bei der Halbraumkonfiguration ist etwas komplizierter. Für die im Raum verteilten notwendigen drei Sensoren lassen sich beliebig viele Umkugeln finden. Hier muss diejenige Umkugel $U$ betrachtet werden, deren Mittelpunkt $\mathbf{x}_{M}$ auf der Oberfläche $z=0$ liegt. Mit dem Mittelpunkt $\mathbf{x}_{M}=\left(x_{M}, y_{M}\right)^{T}$ gilt dann ebenfalls (3.64). Der Nachweis für die Halbraumkonfiguration lässt sich analog zum Fall der Ebenenkonfiguration führen.

\subsubsection{Eigenschaften von wahrem und scheinbarem Skalierungsfaktor}

Jedem wahren und scheinbaren Quellort ist auch ein wahrer und ein scheinbarer Skalierungsfaktor $\gamma$ zugeordnet. Um deren Eigenschaften zu untersuchen, wird ohne Einschränkung erneut ein Koordinatensystem gewählt, das seinen Ursprung im Mittelpunkt des Umkreises bzw. der Umkugel der Sensoren hat. Damit gilt $\mathbf{x}_{M}=\mathbf{0}$, und eine Partikulärlösung für (3.44) ist $\boldsymbol{\theta}_{p}=\mathbf{0}$. Mit (3.46) und $\boldsymbol{\theta}=\left(\mathbf{x}_{0}, 1 / \gamma^{2}, \mathbf{x}_{0}^{T} \mathbf{x}_{0}\right)^{T}$ lassen sich die Beziehungen

$$
\begin{aligned}
& \frac{1}{\gamma_{I}^{2}}=\lambda_{I} \cdot \theta_{h, n+1} \\
& \frac{1}{\gamma_{I I}^{2}}=\lambda_{I I} \cdot \theta_{h, n+1}
\end{aligned}
$$

und

$$
\begin{aligned}
\mathbf{x}_{0, I} & =\lambda_{I} \cdot \boldsymbol{\theta}_{h, 1: n} \\
\mathbf{x}_{0, I I} & =\lambda_{I I} \cdot \boldsymbol{\theta}_{h, 1: n}
\end{aligned}
$$

ableiten. Damit gilt mit $\mathbf{x}_{M}=\mathbf{0}$

$$
\frac{\gamma_{I I}^{2}}{\gamma_{I}^{2}}=\frac{\left\|\mathbf{x}_{0, I}\right\|_{2}}{\left\|\mathbf{x}_{0, I I}\right\|_{2}}=\frac{\lambda_{I}}{\lambda_{I I}}
$$

und für beliebiges $\mathbf{x}_{M}$ folgt

$$
\frac{\gamma_{I I}^{2}}{\gamma_{I}^{2}}=\frac{\left\|\mathbf{x}_{0, I}-\mathbf{x}_{M}\right\|_{2}}{\left\|\mathbf{x}_{0, I I}-\mathbf{x}_{M}\right\|_{2}}
$$




\subsubsection{Konsequenzen für die Sensorplatzierung}

Bei der Quellenlokalisierung auf der Basis skalierbarer Sensor-Quell-Abstände werden bei minimaler Sensoranzahl $m$ zwei Lösungen, eine wahre und eine scheinbare Lösung, erhalten. Die beiden Lösungen $\mathbf{x}_{0, I}, \mathbf{x}_{0, I I}$ mit $\gamma_{I}, \gamma_{I I}$ haben die Eigenschaft, dass sie für jeden Sensor $i$ auf den jeweils gleichen skalierbaren Sensor-Quell-Abstand $\varrho_{i}$ führen. Mit $\varrho_{i}=\gamma \cdot\left\|\mathbf{x}_{i}-\mathbf{x}_{0}\right\|_{2}$ folgt daraus

$$
\gamma_{I} \cdot\left\|\mathbf{x}_{i}-\mathbf{x}_{0, I}\right\|_{2}=\gamma_{I I} \cdot\left\|\mathbf{x}_{i}-\mathbf{x}_{0, I I}\right\|_{2} \quad i=1, \ldots, m
$$

Um die wahre Lösung von der scheinbaren unterscheiden zu können, ist ein weiterer Sensor notwendig. Abbildung 3.9 zeigte jedoch für die Ebenenkonfiguration, dass die wahre und die scheinbare Lösung identische stationäre Konzentrationen auf dem Umkreis der Sensoren hervorrufen.

Dies lässt vermuten, dass ein zusätzlicher Sensor auf dem Umkreis der anderen Sensoren keine zusätzliche Information zur Unterscheidung zwischen wahrem und scheinbarem Quellort liefert. Analoges sollte auch für die Raum- bzw. Halbraumkonfiguration bezüglich der Umkugel der Sensoren gelten. Die Vermutung erweist sich als richtig, was nachfolgend auf der Basis der allgemeinen Lösung (3.44) und (3.45) nachgewiesen wird.

Der Nachweis stützt sich auf die folgende Überlegung: Ein zusätzlicher Sensor liefert dann keine neue Information zur Unterscheidung zwischen wahrem und scheinbarem Quellort, wenn er (3.69) erfüllt, weil in diesem Fall beide möglichen Quellorte $\mathbf{x}_{0, I}$ und $\mathbf{x}_{0, I I}$ auf den gleichen skalierbaren Sensor-Quell-Abstand führen. Nachfolgend wird gezeigt, für welche $\mathbf{x}=\mathbf{x}_{i}$ die Gleichung (3.69) erfüllt ist.

Für den Nachweis wird erneut das Koordinatensystem so gewählt, dass der Ursprung im Mittelpunkt des Umkreises bzw. der Umkugel der Sensoren liegt. Daraus folgt $\mathbf{x}_{M}=\mathbf{0}$ und $\boldsymbol{\theta}_{p}=\mathbf{0}$. Für alle Punkte $\mathbf{x}$, die (3.69) erfüllen, gilt damit

$$
\gamma_{I} \cdot\left\|\mathbf{x}-\lambda_{I} \cdot \boldsymbol{\theta}_{h, 1: n}\right\|_{2}=\gamma_{I I} \cdot\left\|\mathbf{x}-\lambda_{I I} \cdot \boldsymbol{\theta}_{h, 1: n}\right\|_{2}
$$

Umsortieren der Faktoren, Quadrieren und Auflösen der euklidischen Norm führt auf

$$
\frac{\gamma_{I}^{2}}{\gamma_{I I}^{2}}=\frac{\mathbf{x}^{T} \mathbf{x}-2 \lambda_{I I} \mathbf{x}^{T} \boldsymbol{\theta}_{h, 1: n}+\lambda_{I I}^{2} \boldsymbol{\theta}_{h, 1: n}^{T} \boldsymbol{\theta}_{h, 1: n}}{\mathbf{x}^{T} \mathbf{x}-2 \lambda_{I} \mathbf{x}^{T} \boldsymbol{\theta}_{h, 1: n}+\lambda_{I}^{2} \boldsymbol{\theta}_{h, 1: n}^{T} \boldsymbol{\theta}_{h, 1: n}}
$$

Mit (3.67) und den aus (3.61) abgeleiteten Substitutionen

$$
\lambda_{I} \boldsymbol{\theta}_{h, 1: n}^{T} \boldsymbol{\theta}_{h, 1: n}=\frac{r_{u}}{\lambda_{I I}} \quad \text { und } \quad \lambda_{I I} \boldsymbol{\theta}_{h, 1: n}^{T} \boldsymbol{\theta}_{h, 1: n}=\frac{r_{u}}{\lambda_{I}}
$$


folgt

$$
\frac{\gamma_{I}^{2}}{\gamma_{I I}^{2}}=\frac{\lambda_{I I}}{\lambda_{I}}=\frac{\mathbf{x}_{i}^{T} \mathbf{x}_{i}-2 \cdot \lambda_{I I} \cdot \mathbf{x}_{i}^{T} \boldsymbol{\theta}_{h, 1: n}+\lambda_{I I} \cdot \frac{r_{U}^{2}}{\lambda_{I}}}{\mathbf{x}_{i}^{T} \mathbf{x}_{i}-2 \cdot \lambda_{I} \cdot \mathbf{x}_{i}^{T} \boldsymbol{\theta}_{h, 1: n}+\lambda_{I} \cdot \frac{r_{U}^{2}}{\lambda_{I I}}}
$$

Durch Multiplizieren mit den Nennern und Vereinfachen wird daraus

$$
\lambda_{I}\left(\left\|\mathbf{x}_{i}\right\|_{2}^{2}-r_{U}^{2}\right)=\lambda_{I I}\left(\left\|\mathbf{x}_{i}\right\|_{2}^{2}-r_{U}^{2}\right)
$$

Da i. Allg. $\lambda_{I} \neq \lambda_{I I}$ ist, wird Gleichung (3.74) für alle $\mathbf{x}$ erfüllt, für die $\|\mathbf{x}\|_{2}^{2}=r_{U}^{2}$ gilt. Das sind aber alle Standorte, die sich auf dem Umkreis bzw. der Umkugel $U$ befinden.

Damit ist gezeigt, dass durch zusätzliche Sensoren, die auf der Umkugel (dem Umkreis) liegen, keine Unterscheidung zwischen wahrem und scheinbarem Quellort möglich ist. Ein möglicher Standort für den zusätzlichen Sensor ist der Mittelpunkt der Umkugel (des Umkreises), da sich dieser nicht auf der Umkugel (dem Umkreis) befindet.

\subsubsection{Zur Existenz von Lösungen im gestörten Fall}

Die in den vorangegangenen Abschnitten gezeigten Lösungen für den ungestörten Fall bei minimaler Sensoranzahl lassen sich prinzipiell auch für den gestörten Fall nutzen. Dabei sind für die skalierbaren Sensor-Quell-Abstände die Schätzwerte $\hat{\varrho}_{i}$ zu verwenden. Allerdings können die Störungen zu solchen Werten von $\hat{\varrho}_{i}$ führen, für die keine Lösung für den Quellort existiert. Nachfolgend werden Kriterien zur Existenz von Lösungen im gestörten Fall bei Verwenden minimaler Sensoranzahl hergeleitet.

Für die Ebenenkonfiguration wurde im Abschnitt 3.2.3 gezeigt, dass sich der wahre und der scheinbare Quellort als Schnittpunkte der Schnittkreise mit den Mittelpunkten $\left(x_{S, 12}, y_{S, 12}\right)^{T},\left(x_{S, 13}, y_{S, 13}\right)^{T}$ und den Radien $r_{S, 12}, r_{S, 13}$ auffassen lassen. Im ungestörten Fall besitzen diese Schnittkreise stets mindestens einen Schnittpunkt, nämlich den wahren Quellort. Zudem existiert bei minimaler Sensoranzahl im ungestörten Fall i. Allg. ein zweiter, scheinbarer Quellort.

Im gestörten Fall können jedoch solche Werte für $\hat{\varrho}_{i}$ geschätzt werden, für die die zugehörigen Schnittkreise keinen Schnittpunkt besitzen. Dann hat auch die Quellenlokalisierung auf der Basis minimaler Sensoranzahl keine Lösung. Werden die Schnittkreise betrachtet, existiert dann eine Lösung, wenn der Abstand der Mittelpunkte der Schnittkreise kleiner oder gleich der Summe der Radien der Schnittkreise ist, also wenn gilt

$$
\sqrt{\left(x_{S, 12}-x_{S, 13}\right)^{2}+\left(y_{S, 12}-y_{S, 13}\right)^{2}} \leq r_{S, 12}+r_{S, 13}
$$


Bei der Raumkonfiguration ist eine geometrische Interpretation über Schnittkugeln formelmäßig schwer zu fassen. Die Existenz von Lösungen lässt sich aber über (3.48) analysieren. Eine Lösung existiert genau dann, wenn die Diskriminante die Bedingung

$$
\left(\frac{2 \cdot \boldsymbol{\theta}_{p, 1: n}^{T} \cdot \boldsymbol{\theta}_{h, 1: n}-\theta_{h,(n+2)}}{2 \cdot \boldsymbol{\theta}_{h, 1: n}^{T} \cdot \boldsymbol{\theta}_{h, 1: n}}\right)^{2}-\frac{\boldsymbol{\theta}_{p, 1: n}^{T} \cdot \boldsymbol{\theta}_{p, 1: n}+\theta_{p,(n+2)}}{\boldsymbol{\theta}_{h, 1: n}^{T} \cdot \boldsymbol{\theta}_{h, 1: n}} \geq 0
$$

erfüllt. Diese Bedingung ist auch für die Ebenen- und Halbraumkonfiguration anwendbar.

\subsection{Ermittlung des Quellorts im gestörten Fall}

Im vorangegangenen Abschnitt wurde das Problem der Quellortbestimmung auf der Basis skalierbarer Sensor-Quell-Abstände bei minimaler Sensoranzahl $m$ für den ungestörten Fall behandelt. Es wurde gezeigt, dass für die hergeleiteten Verfahren im Fall von Störungen keine Lösung für den Quellort existieren muss. Selbst wenn eine Lösung existiert, hat die Verwendung der minimalen Anzahl von Sensoren den Nachteil, dass die Schätzfehler von $\hat{\varrho}_{i}$ direkt eine Abweichung zwischen tatsächlichem Quellort $\mathbf{x}_{0}$ und geschätztem Quellort $\hat{\mathbf{x}}_{0}$ bewirken, da bei minimaler Sensoranzahl kein Fehlerausgleich möglich ist. Deshalb ist es sinnvoll eine größere Anzahl von Sensoren $p>m$ zu verwenden. Diese erlauben bei richtiger Anordnung zum einen die Unterscheidung zwischen wahrem und scheinbarem Quellort und zum anderen einen Fehlerausgleich.

Die Betrachtung der minimalen Sensoranzahl führte im ungestörten Fall jeweils auf die Lösung eines algebraischen Gleichungssystems (pro Sensor eine Gleichung). Bei minimaler Sensoranzahl ist dieses Gleichungssystems auch im gestörten Fall konsistent, wenn eine Lösung existiert. Anders verhält es sich, wenn die Sensoranzahl auf $p>m$ steigt. Durch die neu hinzukommenden Gleichungen wird ein überbestimmtes, im gestörten Fall inkonsistentes Gleichungssystem erhalten. Das bedeutet, dass es für kein $\gamma$ einen gemeinsamen Schnittpunkt aller $\mathcal{P}_{i}$ (Kugeln/Kreise) gibt, wie Abb. 3.13 für den Fall einer Ebenenkonfiguration verdeutlicht.

Ein pragmatischer Ansatz zur Bestimmung des Quellorts besteht darin, konsistente Gleichungssysteme für jeweils $m$ Sensoren aufzustellen und zu lösen. Der Mittelwert aus den erhaltenen Quellorten stellt dann einen Schätzwert für den Quellort dar. 


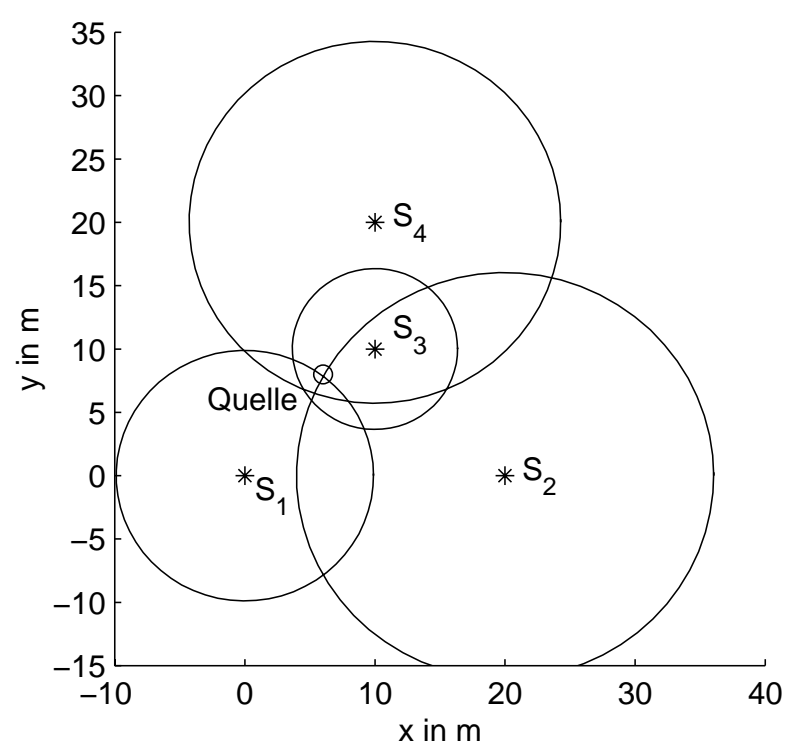

Abb. 3.13: Mengen $\mathcal{P}_{i}$ (Kreise) für alle $p$ Sensoren, die im gestörten Fall auch für optimales $\gamma$ keinen gemeinsamen Schnittpunkt besitzen.

Besser ist es jedoch, für das überbestimmte Gleichungssystem ein Ausgleichsproblem zu formulieren und zu lösen. Dabei wird ein solcher Parametersatz $\left(\hat{\mathbf{x}}_{0}, \hat{\gamma}\right)$ bestimmt, für den sich die kleinste Summe der Quadrate der Abstände zwischen $\hat{\mathbf{x}}_{0}$ zu allen $\mathcal{P}_{i}$ (Kreisen/Kugeln) ergibt

$$
\sum_{i=1}^{p}\left(\left\|\mathbf{x}_{i}-\mathbf{x}_{0}\right\|_{2}-\frac{\hat{\varrho}_{i}}{\gamma}\right)^{2} \stackrel{!}{=} \underset{\mathbf{x}_{0}, \gamma}{\operatorname{Min}}
$$

Dieses Optimierungsproblem, das den so genannten geometrischen Fehler minimiert, lässt sich nur iterativ lösen. In Hinsicht auf die numerische Lösung bieten Optimierungsprobleme, die hingegen den algebraischen Fehler ${ }^{5}$ minimieren, Vorteile. Das zugeordnete algebraische Problem lautet

$$
\sum_{i=1}^{p}\left(\frac{\hat{\varrho}_{i}^{2}}{\gamma^{2}}-\left\|\mathbf{x}_{i}-\mathbf{x}_{0}\right\|_{2}^{2}\right)^{2} \stackrel{!}{=} \underset{\mathbf{x}_{0}, \gamma}{\operatorname{Min}} .
$$

Im ungestörten Fall sind die Lösungen für (3.77) und (3.78) identisch, da der Fehler für jeden Sensor null wird. Im gestörten Fall unterscheiden sich i. Allg. die Lösungen für (3.77) und (3.78), da die Sensor-Quell-Abstände linear bzw. quadratisch eingehen. Infolge des Quadrierens werden die Fehler großer Kreise überbewertet.

Einen Ausweg bietet eine sensorspezifische Wichtung $g_{i}$

$$
\sum_{i=1}^{p}\left(g_{i}\left(\frac{\hat{\varrho}_{i}^{2}}{\gamma^{2}}-\left\|\mathbf{x}_{i}-\mathbf{x}_{0}\right\|_{2}^{2}\right)\right)^{2} \stackrel{!}{=} \min _{\mathbf{x}_{0}, \gamma}
$$

\footnotetext{
${ }^{5}$ Der algebraische Fehler resultiert aus dem Gleichungsfehler der impliziten Kreisgleichung. Hingegen betrachtet der geometrische Fehler die geometrischen Abstände zum Kreis.
} 
Zur Herleitung der Gewichte $g_{i}$ sind für einen Sensor $i$ geometrischer und algebraischer Fehler in Abb. 3.14 illustriert.

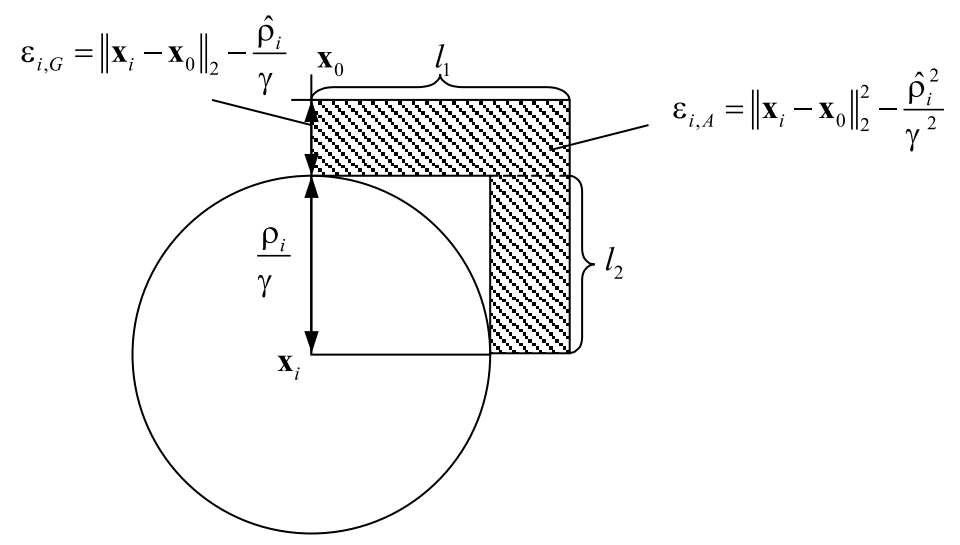

Abb. 3.14: Beziehung zwischen geometrischem Fehler $\varepsilon_{i, G}$ und algebraischem Fehler $\varepsilon_{i, A}$.

Abbildung 3.14 macht deutlich, dass der geometrische Fehler $\varepsilon_{i, G}$ dem Abstand zwischen Quellort und Kreis entspricht, während der algebraische Fehler $\varepsilon_{i, A}$ die schraffierte Fläche repräsentiert. Für kleine Abstände zwischen Quellort und Kreis gilt jedoch

$$
l_{1}+l_{2} \approx 2 \cdot l_{2}=\frac{2 \hat{\varrho}_{i}}{\gamma}
$$

und es ist in Näherung

$$
\varepsilon_{i, G}=\frac{\varepsilon_{i, A}}{l_{1}+l_{2}} \approx \frac{\varepsilon_{i, A}}{2 \cdot l_{2}} .
$$

Der geometrische Fehler entspricht in etwa dem gewichteten algebraischen Fehler

$$
\left(\frac{\underline{\varrho}_{i}}{\gamma}-\left\|\mathbf{x}_{i}-\mathbf{x}_{0}\right\|_{2}\right) \approx \frac{\gamma}{2 \hat{\varrho}_{i}}\left(\frac{\hat{\varrho}_{i}^{2}}{\gamma^{2}}-\left\|\mathbf{x}_{i}-\mathbf{x}_{0}\right\|_{2}^{2}\right) .
$$

Die Zielfunktion (3.79) schreibt sich mit dieser speziellen Wichtung als

$$
\sum_{i=1}^{p}\left(\frac{1}{\hat{\varrho}_{i}} \cdot\left(\frac{\hat{\varrho}_{i}^{2}}{\gamma^{2}}-\left\|\mathbf{x}_{i}-\mathbf{x}_{0}\right\|_{2}^{2}\right)\right)^{2} \stackrel{!}{=} \min _{\mathbf{x}_{0}, \gamma}
$$

Der Faktor $\gamma / 2$ wird in der Wichtung weggelassen, da er keinen Einfluss auf die Optimallösung hat.

Werden zur Lösung von (3.83) A, b und $\boldsymbol{\theta}$ analog (3.45) (Abschnitt 3.2.6) gewählt

$$
\mathbf{A}=\left(\begin{array}{ccc}
\frac{2 \mathbf{x}_{1}^{T}}{\hat{\varrho}_{1}} & \hat{\varrho}_{1} & \frac{-1}{\hat{\varrho}_{1}} \\
\vdots & \vdots & \vdots \\
\frac{2 \mathbf{x}_{p}^{T}}{\hat{\varrho}_{p}} & \hat{\varrho}_{p} & \frac{-1}{\hat{\varrho}_{p}}
\end{array}\right), \quad \boldsymbol{\theta}=\left(\begin{array}{c}
\mathbf{x}_{0} \\
\frac{1}{\gamma^{2}} \\
\mathbf{x}_{0}^{T} \mathbf{x}_{0}
\end{array}\right), \quad \mathbf{b}=\left(\begin{array}{c}
\frac{\mathbf{x}_{1}^{T} \mathbf{x}_{1}}{\hat{\varrho}_{1}} \\
\vdots \\
\frac{\mathbf{x}_{p}^{T} \mathbf{x}_{p}}{\hat{\varrho}_{p}}
\end{array}\right)
$$


lässt sich ein Least-squares-Problem

$$
\|\mathbf{A} \boldsymbol{\theta}-\mathbf{b}\|_{2}^{2} \stackrel{!}{=} \operatorname{Min}_{\boldsymbol{\theta}} \text { mit quadratischer Restriktion } \theta_{n+2}=\boldsymbol{\theta}_{1: n}^{T} \boldsymbol{\theta}_{1: n}
$$

für $\mathbf{x}_{0}$ und $\gamma$ formulieren.

In [41, 42] wird ein Algorithmus für eine quasi geschlossene Lösung solcher quadratisch restringierten Least-squares-Probleme beschrieben. Bis auf die Suche der Wurzeln eines Polynoms sind alle Berechnungen nicht-iterativ, was zu einem geringen Rechenaufwand des Verfahrens führt. Für den Fall minimaler Sensoranzahl $(p=m)$ oder ungünstiger Sensorstandorte (Sensoren liegen alle auf gemeinsamem Kreis), bei denen die zusätzlichen Sensoren keine weitere Information bezüglich der Quellenlokalisierung beisteuern (vgl. Abschnitt 3.3.3), liefert der Algorithmus sowohl die wahre als auch die scheinbare Lösung (vgl. Abschnitt 3.8).

\subsection{Wichtung}

Durch (3.77) wird die Minimierung der Summe der Abstandsquadrate vom geschätzten Quellort zu allen Kreisen im geometrischen Sinne gefordert. Das gewichtete algebraische Problem (3.78) liefert hierzu eine gut lösbare Näherung. Der Ausgleich erfolgt bei diesem Problem offensichtlich in der Weise

$$
\sum_{i=1}^{p} \varepsilon_{i, G}^{2} \stackrel{!}{=} \underset{\mathbf{x}_{0}, \gamma}{\operatorname{Min}} .
$$

Damit werden die Fehler $\varepsilon_{i, G}$ für jeden Sensor $i$ gleich bewertet. Allerdings handelt es sich bei $\varepsilon_{i, G}$ um einen Fehler, der den Konzentrationsmessfehler von Sensor $i$ nicht direkt beschreibt, sondern nur mittelbar (nichtlinear) durch diesen Fehler entsteht. Daher ist es sinnvoller, einen Ausgleich in den Konzentrationen $C_{i}$ gemäß

$$
\sum_{i=1}^{p}\left(\Delta C_{i}\right)^{2} \stackrel{!}{=} \operatorname{Min}_{\mathbf{x}_{0}, \gamma}
$$

anzustreben. In diesem Fall werden die Abweichungen $\Delta C_{i}$ für jeden Sensor $i$ gleich bewertet. Ein solcher Ausgleich wird durch das einstufige Ausgangsfehlerverfahren erzielt. Er ist bei stationären unkorrelierten normalverteilten Fehlern (Störungen) sowie identischer Streuung der Konzentrationsmessungen um den jeweiligen wahren Konzentrationswert optimal bezüglich der Streuung der geschätzten Quellorte ${ }^{6}$.

\footnotetext{
${ }^{6} \mathrm{Im}$ Fall von stationären unkorrelierten normalverteilten Störungen stellt die ausgangsfehlerbasierte Schätzung eine Maximum-likelihood-Schätzung dar und erreicht die untere Cramer-Rao-Schranke [104]. Diese Schranke gibt die kleinste mögliche Streuung jeder bias-freien Schätzung an.
} 
Lassen sich Wichtungsfaktoren $w_{i}$ finden, für die in Näherung

$$
\Delta C_{i} \approx w_{i} \cdot \Delta \varepsilon_{i, G}
$$

gilt, kann eine mit $w_{i}$ gewichtete Version von (3.83)

$$
\sum_{i=1}^{p}\left(\frac{w_{i}}{\hat{\varrho}_{i}} \cdot\left(\frac{\hat{\varrho}_{i}^{2}}{\gamma^{2}}-\left\|\mathbf{x}_{i}-\mathbf{x}_{0}\right\|_{2}^{2}\right)\right)^{2} \stackrel{!}{=} \operatorname{Min}_{\mathbf{x}_{0}, \gamma}
$$

genutzt werden, um einen Ausgleich in $C_{i}$ zu erhalten. Die zugeordnete Optimallösung ist dann näherungsweise optimal bezüglich der Streuung der geschätzten Quellorte. Die Güte der Quellortschätzung entspricht dann etwa der des Ausgangsfehlerverfahrens, ohne dessen numerische Probleme (Nebenminima) zu besitzen.

Solche Wichtungsfaktoren lassen sich allerdings nur für den Fall stationärer Konzentrationen herleiten. Mit (3.5) und (3.6) gilt

$$
\frac{\hat{\varrho}_{i}}{\gamma}=\frac{q_{0}}{4 \pi K C_{i}}
$$

Über die klassische Fehlerrechnung [45] (Ableiten $\mathrm{d} \frac{\hat{\underline{\rho}}_{i}}{\gamma} / \mathrm{d} C_{i}$, Ausnutzen von $\mathrm{d} \varepsilon_{i, G}=\mathrm{d} \frac{\hat{\underline{\varrho}}_{i}}{\gamma}$, Ersetzen der Differenziale durch Differenzen) werden

$$
\Delta \varepsilon_{i, G} \approx-\frac{q_{0}}{4 \pi K C_{i}^{2}} \cdot \Delta C_{i}
$$

und damit die optimalen Wichtungsfaktoren

$$
w_{i}=-C_{i}^{2} \cdot \frac{4 \pi K}{q_{0}}
$$

erhalten $^{7}$. Da der Faktor $-\frac{4 \pi K}{q_{0}}$ nur eine Skalierung aller Fehler bewirkt, kann er bei der Optimierung weggelassen werden. Mit den Wichtungsfaktoren und der Ersetzung $1 / \hat{\varrho}_{i}:=C_{i}$ vgl. (3.5) lautet das gewichtete algebraische Optimierungsproblem

$$
\sum_{i=1}^{p}\left(\frac{1}{\hat{\varrho}_{i}^{3}} \cdot\left(\frac{\hat{\varrho}_{i}^{2}}{\gamma^{2}}-\left\|\mathbf{x}_{i}-\mathbf{x}_{0}\right\|_{2}^{2}\right)\right)^{2} \stackrel{!}{=} \underset{\mathbf{x}_{0}, \gamma}{\operatorname{Min}} .
$$

Daraus folgt das restringierte Least-squares-Problem

$$
\|\mathbf{A} \boldsymbol{\theta}-\mathbf{b}\|_{2}^{2} \stackrel{!}{=} \operatorname{Min}_{\boldsymbol{\theta}} \quad \text { mit der Restriktion } \quad \theta_{n+2}=\boldsymbol{\theta}_{1: n}^{T} \boldsymbol{\theta}_{1: n}
$$

und

$$
\mathbf{A}=\left(\begin{array}{ccc}
\frac{2 \mathbf{x}_{1}^{T}}{\hat{\varrho}_{1}^{3}} & \frac{1}{\hat{\varrho}_{1}} & \frac{-1}{\hat{\varrho}_{1}^{3}} \\
\vdots & \vdots & \vdots \\
\frac{2 \mathbf{x}_{p}^{T}}{\hat{\varrho}_{p}^{3}} & \frac{1}{\hat{\varrho}_{p}} & \frac{-1}{\hat{\varrho}_{p}^{3}}
\end{array}\right), \quad \boldsymbol{\theta}=\left(\begin{array}{c}
\mathbf{x}_{0} \\
\frac{1}{\gamma^{2}} \\
\mathbf{x}_{0}^{T} \mathbf{x}_{0}
\end{array}\right), \quad \mathbf{b}=\left(\begin{array}{c}
\frac{\mathbf{x}_{1}^{T} \mathbf{x}_{1}}{\hat{\varrho}_{1}^{3}} \\
\vdots \\
\frac{\mathbf{x}_{p}^{T} \mathbf{x}_{p}}{\hat{\varrho}_{p}^{3}}
\end{array}\right)
$$

das bis auf die Belegung der Matrix A und des Vektors b dem von (3.85) entspricht.

\footnotetext{
${ }^{7}$ Beim Ersetzen der Differenziale durch Differenzen ist das Gleichheitszeichen in ein Näherungszeichen zu ändern.
} 
Beispiel 3.3 Für die in Abb. 3.15 dargestellte Ebenenkonfiguration wird eine Lokalisierung auf der Basis der stationären Konzentrationen durchgeführt. Dabei werden die Konzentrationen mit deterministischen Absolutfehlern beaufschlagt, wie sie durch Kalibrierfehler entstehen. Es wird also zum Konzentrationswert jedes Sensors ein fester positiver bzw. negativer Konzentrationswert (Kalibrierfehler) addiert. Der hier verwendete Fehler bedeutet einen relativen Fehler von $5 \%$ bezogen auf den Messbereich und je nach Standort des Sensors einen relativen Fehler von bis zu $20 \%$ bezogen auf den ungestörten Konzentrationsmesswert. Alle 16 möglichen Kombinationen, die sich für zwei verschiedene Kalibrierfehler pro Sensor für die hier betrachteten 4 Sensoren ergeben, werden simuliert. Die Ergebnisse, die dabei sowohl mit dem ungewichteten Verfahren, als auch mit dem gewichteten Verfahren erzielt werden, zeigt Abb. 3.15. Zudem sind die Ergebnisse des Ausgangsfehlerverfahrens dargestellt, das hier stets das globale Minimum findet und nicht in Nebenminima abbricht, da die wahren Werte für Quellort und-intensität als Startwerte vorgegeben wurden.

Beispiel 3.3 zeigt, dass mit dem gewichteten Verfahren deutlich bessere Ergebnisse erzielt werden als mit dem ungewichteten Verfahren (maximale Abweichung zwischen Quellort und Quellortschätzung 4.2m ungewichtet, $2.3 \mathrm{~m}$ gewichtet). Im Vergleich zum Ausgangsfehlerverfahren (maximale Abweichung 2.2m) sind keine wesentlichen Unterschiede zu erkennen.

\subsection{Ermittlung der Quellintensität}

\subsubsection{Vorbemerkung}

Nachdem der Quellort $\mathbf{x}_{0}$ sowie der Skalierungsfaktor $\gamma$ geschätzt wurden, lässt sich daraus die Quellintensität $q_{0}$ bestimmen. Praktisch können über die geschätzte Quellintensität beispielsweise Rückschlüsse auf das Ausmaß einer Leckage und damit auf notwendige Gegenmaßnahmen gezogen werden.

Im Gegensatz zur Ermittlung von Quellort $\mathbf{x}_{0}$ und Skalierungsfaktor $\gamma$ auf der Basis skalierbarer Sensor-Quell-Abstände $\hat{\varrho}_{i}$ muss bei der Schätzung von $q_{0}$ unterschieden werden, ob $\hat{\varrho}_{i}$ auf der Basis von stationären oder instationären Konzentrationen geschätzt wurde. 

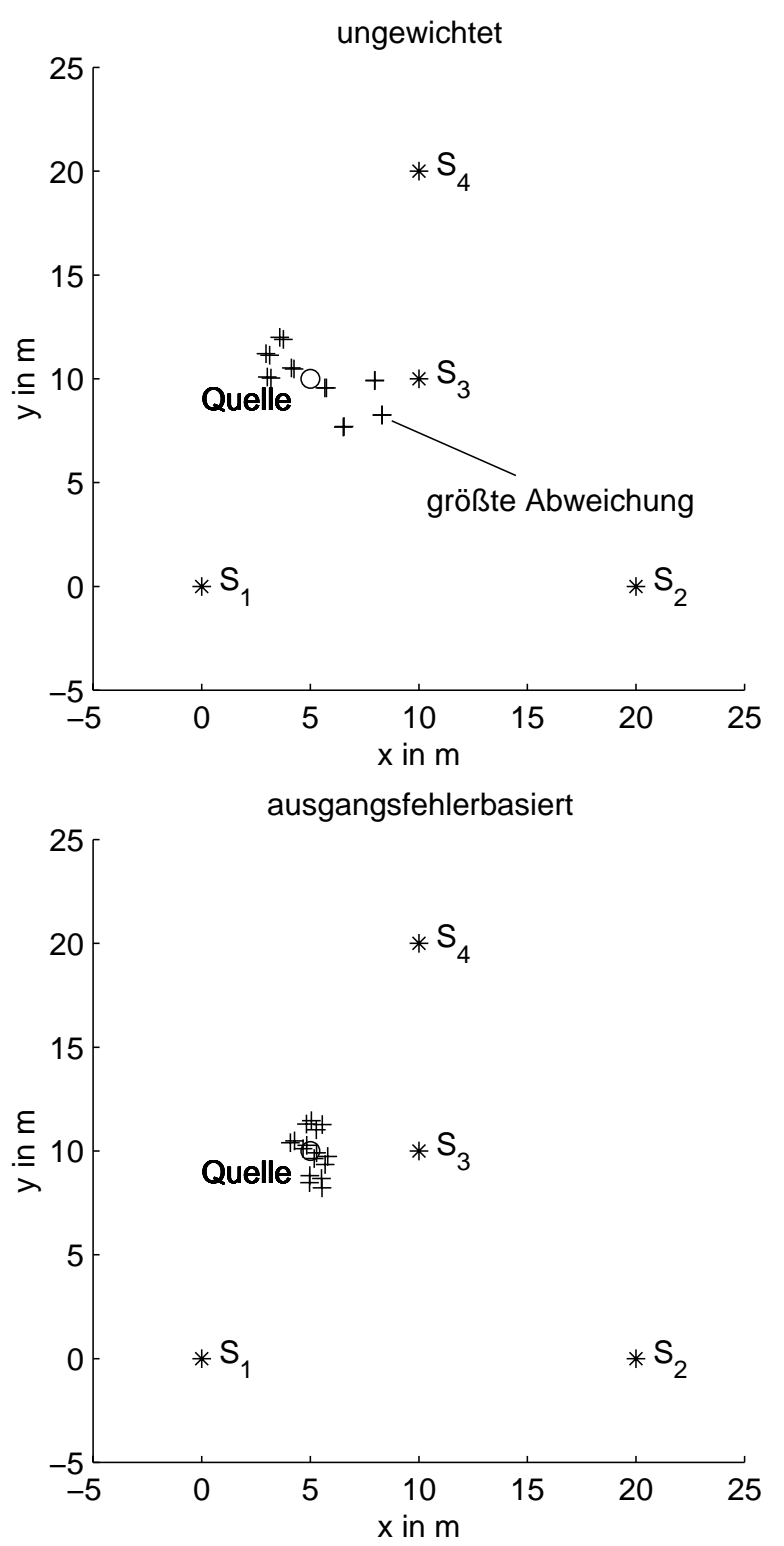

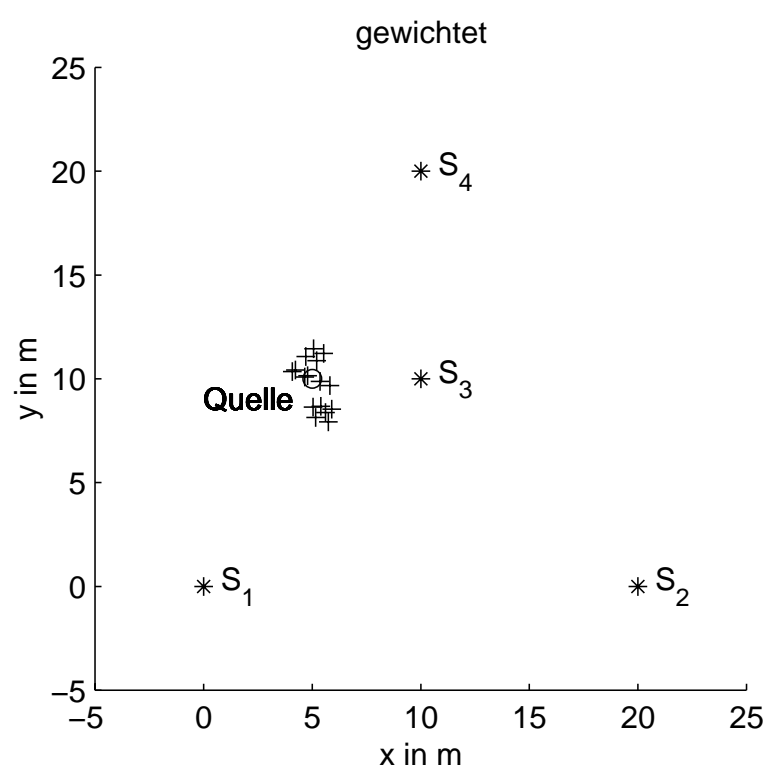

Abb. 3.15: Abweichungen der Quellortschätzung beim ungewichteten Verfahren, beim gewichteten Verfahren und beim ausgangsfehlerbasierten Standardverfahren. (Als Startwerte für das ausgangsfehlerbasierte Standardverfahren wurden die wahren Parameter verwendet; damit wird das globale Minimum gefunden.) Der Schätzwert für den Quellort, der beim ungewichteten Verfahren am weitesten von der Quelle entfernt liegt, entsteht dadurch, dass die Sensoren $S_{2}, S_{3}$ zu große Konzentration messen (positiver Fehler) und die Sensoren $S_{1}, S_{4}$ zu kleine Konzentrationen messen (negativer Fehler).

\subsubsection{Ermittlung der Quellintensität mit stationären Konzentrationen}

Wurden die skalierbaren Sensor-Quell-Abstände auf der Basis stationärer Konzentrationen ermittelt (vgl. Abschnitt 3.1.1), dann gilt gemäß (3.6) für den Skalierungsfaktor

$$
\gamma=\frac{4 \pi K}{q_{0}} \quad \text { bzw. } \quad \gamma=\frac{2 \pi K}{q_{0}} \quad \text { (für das halbunendliche Medium) }
$$

Mit einem Schätzwert $\hat{\gamma}$ lassen sich Diffusionskoeffizient und Quellintensität nicht getrennt voneinander bestimmen. Es kann nur das Verhältnis

$$
\widehat{K / q_{0}}=\frac{\hat{\gamma}}{4 \pi} \quad \text { bzw. } \quad{\widehat{K} / q_{0}}_{1}=\frac{\hat{\gamma}}{2 \pi} \quad \text { für das halbunendliche Medium }
$$

bestimmt werden. 


\subsubsection{Ermittlung der Quellintensität mit instationären Konzentrationen}

Wurden die skalierbaren Sensor-Quell-Abstände auf der Basis instationärer Konzentrationen geschätzt (vgl. Abschnitt 3.1.2), so liegen Schätzwerte $\hat{\alpha}_{i}$ und $\hat{\beta}_{i}$ für jeden Sensor $i$ vor.

Werden die Gleichungen für $\alpha_{i}$ und $\beta_{i}(3.8)$

$$
\alpha_{i}=\frac{q_{0}}{4 \pi K\left\|\mathbf{x}_{i}-\mathbf{x}_{0}\right\|_{2}}, \quad \beta_{i}=\frac{\left\|\mathbf{x}_{i}-\mathbf{x}_{0}\right\|_{2}}{2 \sqrt{K}}
$$

kombiniert, lässt sich mit den Schätzwerten $\hat{\alpha}_{i}, \hat{\beta}_{i}$ und dem geschätzten Quellort $\hat{\mathbf{x}}_{0}$ ein i. Allg. überbestimmtes Gleichungssystem

$$
\underbrace{\left(\begin{array}{cc}
-1 & 4 \pi \hat{\alpha}_{1}\left\|\mathbf{x}_{1}-\hat{\mathbf{x}}_{0}\right\|_{2} \\
\vdots & \vdots \\
-1 & 4 \pi \hat{\alpha}_{p}\left\|\mathbf{x}_{p}-\hat{\mathbf{x}}_{0}\right\|_{2} \\
4 \hat{\beta}_{1}^{2} \\
\vdots \\
\vdots & 4 \hat{\beta}_{p}^{2}
\end{array}\right)}_{\mathbf{A}} \underbrace{\left(\begin{array}{c}
q_{0} \\
K
\end{array}\right)}_{\boldsymbol{\theta}} \cong \underbrace{\left(\begin{array}{c}
\mathbf{0}_{p} \\
\left\|\mathbf{x}_{1}-\hat{\mathbf{x}}_{0}\right\|_{2}^{2} \\
\vdots \\
\left\|\mathbf{x}_{p}-\hat{\mathbf{x}}_{0}\right\|_{2}^{2}
\end{array}\right)}_{\mathbf{b}} .
$$

generieren. Wird daraus das Ausgleichsproblem

$$
\|\mathbf{A} \boldsymbol{\theta}-\mathbf{b}\|_{2}^{2} \stackrel{!}{=} \underset{\boldsymbol{\theta}}{\operatorname{Min}} v
$$

formuliert, folgt die geschlossene Lösung

$$
\hat{\boldsymbol{\theta}}=\left(\mathbf{A}^{T} \mathbf{A}\right)^{-1} \mathbf{A}^{T} \mathbf{b} .
$$

\subsection{Fehler der Quellortschätzung mit stationären Konzentrationen}

In diesem Abschnitt wird an einem Simulationsbeispiel untersucht, welche Abweichungen bei der Quellenlokalisierung auf der Basis stationärer Konzentrationen entstehen, wenn als Schätzwerte für die stationären Konzentrationen Messwerte nach einer großen, aber endlichen Messzeit verwendet werden. Die Untersuchungen basieren auf den sich entsprechend Beispiel 1.2 ergebenden ungestörten Konzentrationsverläufen. 
Werden die zu einem bestimmten Zeitpunkt gemessenen Konzentrationen $C_{i, k}$ als Schätzwerte für $C\left(\mathbf{x}_{i}, \infty\right)$ angesehen, entsteht ein systematischer Fehler, da die Konzentrationen tatsächlich noch steigen. Damit ergibt sich auch im ungestörten Fall eine Abweichung zwischen tatsächlichem und geschätztem Quellort, wenn das stationäre Verfahren verwendet wird. Diese zeitabhängige Abweichung ist in Abb. 3.16 für verschiedene Diffusionskoeffizienten $K$ dargestellt.

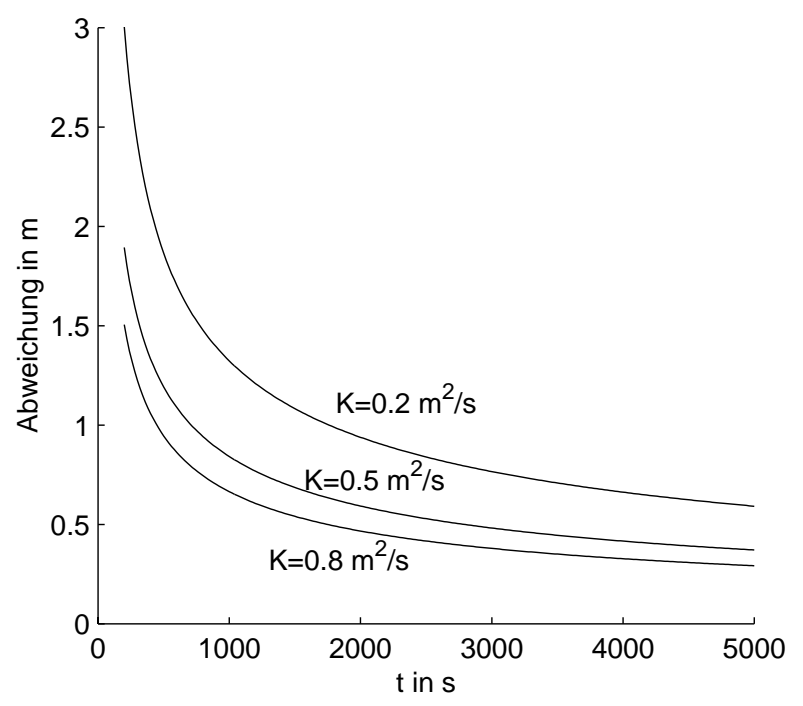

Abb. 3.16: Abweichung des geschätzten Quellorts vom wahren Wert in Abhängigkeit von der Beobachtungszeit bei Schätzung auf der Basis stationärer Endwerte im ungestörten Fall.

Die Abweichung zwischen wahrem und geschätztem Quellort sinkt erwartungsgemäß mit der Zeit, da sich die Konzentrationen den stationären Werten nähern. Jedoch ist sie bei niedrigen Diffusionskoeffizienten auch lange Zeit nach Emissionsbeginn noch relativ hoch (in Abbildung 3.16 nach einer Stunde und $K=0.2 \mathrm{~m}^{2} / \mathrm{s}$ ca. $0.8 \mathrm{~m}$ Abweichung). Die notwendige Zeit verkleinert sich jedoch mit steigendem Diffusionskoeffizienten $K$. Im Gegensatz dazu findet das auf der instationären Lösung der Ausbreitungsgleichung basierende Verfahren im ungestörten Fall den richtigen Quellort, sobald drei Konzentrationsmesswerte pro Sensor vorliegen (drei Parameter pro Sensor).

\subsection{Probleme bei ungünstigen Sensorstandorten}

In Abschnitt 3.3.3 wurde gezeigt, dass für eine eindeutige Lösung für die Quellenlokalisierung bei der Ebenenkonfiguration ein zusätzlicher, vierter Sensor bzw. bei der Raumkonfiguration ein fünfter Sensor notwendig ist, der sich nicht auf dem Umkreis bzw. auf der Umkugel der anderen Sensoren befinden darf. Doch selbst wenn diese Forderung erfüllt 
ist, können Probleme bei der Unterscheidung zwischen wahrem und scheinbarem Quellort auftreten. Das ist dann der Fall, wenn der Sensor nicht weit genug vom Umkreis bzw. von der Umkugel entfernt liegt und der Messfehler (z. B. durch Kalibrierfehler) an diesem Sensor dazu führt, dass der Konzentrationsmesswert näher dem Konzentrationsprofil des scheinbaren Quellorts liegt als dem des wahren Quellorts (vgl. Abb. 3.9). Das nachfolgende Beispiel verdeutlicht dies.

Beispiel 3.4 Für den Fall einer Ebenenkonfiguration mit den Sensorstandorten $\mathbf{x}_{1}=$ $(0 \mathrm{~m}, 0 \mathrm{~m})^{T}, \mathbf{x}_{2}=(10 \mathrm{~m}, 0 \mathrm{~m})^{T}, \mathbf{x}_{3}=(0 \mathrm{~m}, 10 \mathrm{~m})^{T}, \mathbf{x}_{4}=(9 \mathrm{~m}, 9 \mathrm{~m})^{T}$ soll eine Quelle bei $\mathbf{x}_{0}=$ $(3 \mathrm{~m}, 9 \mathrm{~m})^{T}$ mit der Intensität $q_{0}=100 \mathrm{mg} / \mathrm{s}$ auf der Basis der gemessenen ungestörten stationären Konzentrationen $C_{1}=8.39 \mathrm{mg} / \mathrm{m}^{3}, C_{2}=6.98 \mathrm{mg} / \mathrm{m}^{3}, C_{3}=25.17 \mathrm{mg} / \mathrm{m}^{3}$ und $C_{4}=13.26 \mathrm{mg} / \mathrm{m}^{3}$ lokalisiert werden (Abb. 3.17). Anschließend wird der gestörte Fall betrachtet, bei dem Sensor 4 einen Messfehler von $10 \%$ aufweist $\left(C_{4}=11.94 \mathrm{mg} / \mathrm{m}^{3}\right)$.

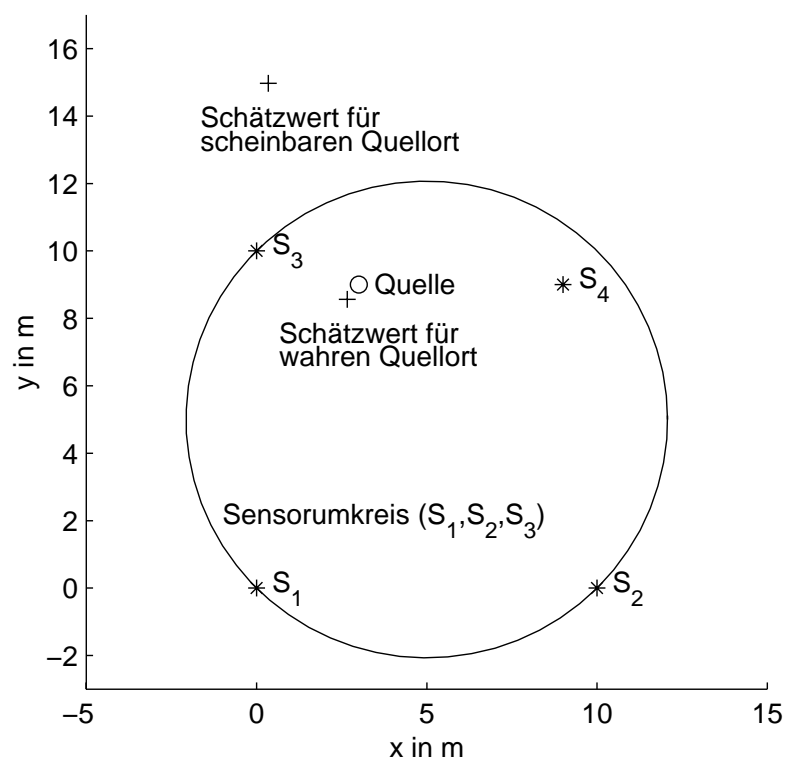

Abb. 3.17: Probleme bei ungünstiger Sensorkonfiguration. Durch Störungen kann das Nebenminimum zum globalen Minimum werden. Damit wird der scheinbare und nicht der wahre Quellort gefunden.

Im ungestörten Fall lässt sich der Quellort eindeutig bestimmen, da sich der vierte Sensor $S_{4}$ nicht auf dem Umkreis der anderen drei Sensoren befindet (Abb. 3.17). Sowohl der Gleichungsfehler (3.78) bzw. (gewichteter Gleichungsfehler (3.93)) als auch der Ausgangsfehler (1.1) besitzen ein eindeutiges globales Minimum im wahren Quellort $\mathbf{x}_{0, I}=$ $(3 \mathrm{~m}, 9 \mathrm{~m})^{T}$ (Gleichungsfehler $\left.=0 \mathrm{~m}^{2}\right)$. Die scheinbare Lösung für den Quellort, die bei Verwenden von nur drei Sensoren $S_{1}, S_{2}, S_{3}$ ebenfalls ein globales Minimum darstellt, liegt 
bei $\mathbf{x}_{0, I I} \approx(0 \mathrm{~m}, 15 \mathrm{~m})^{T}$ (am Umkreis gespiegelte Lösung). Durch Hinzunahme des vierten Sensors wird diese scheinbare Lösung nach $\mathbf{x}_{0, I I} \approx(1.7 \mathrm{~m}, 14.5 \mathrm{~m})$ verschoben und zum Nebenminimum (Gleichungsfehler $=0.76 \mathrm{~m}^{2}$ ). Sie kann damit von der wahren Lösung unterschieden werden.

Wird nun die Konzentration $C_{4}$ gestört, so liegt der Schätzwert für den wahren Quellort bei $\hat{\mathbf{x}}_{0, I} \approx(2.7 \mathrm{~m}, 8.6 \mathrm{~m})^{T}$ (Gütewert des Gleichungsfehlers $\left.=0.30 \mathrm{~m}^{2}\right)$ und für den scheinbaren Quellort bei $\hat{\mathbf{x}}_{0, I I} \approx(0.3 \mathrm{~m}, 15 \mathrm{~m})^{T}$ (Gütewert des Gleichungsfehlers $\left.=0.18 \mathrm{~m}^{2}\right)$ (Abb. 3.17). Damit ist der Gütewert des Gleichungsfehlers für den scheinbaren Quellort kleiner als für den wahren Quellort. Gleiches gilt auch für den Gütewert des Ausgangsfehlers. Das heißt, das eindeutige globale Minimum liegt im scheinbaren Quellort.

Das Beispiel zeigt, dass bei einer Sensoranordnung, bei der sich der zusätzliche, vierte Sensor nahe dem Umkreis der anderen drei Sensoren befindet, i. Allg. ein eindeutiges globales Minimum existiert. Bedingt durch kleine Störungen kann das globale Minimum aber im scheinbaren Quellort liegen. Ausgangsfehlerbasierte einstufige Verfahren, wie Gradientenverfahren oder evolutionäre Verfahren [53], finden in Abhängigkeit von den Startwerten der Optimierung stets nur ein Minimum. Selbst wenn das globale Minimum gefunden wird, kann dies den falschen (scheinbaren) Quellort repräsentieren. Das hier verwendete Verfahren zur Minimierung des Gleichungsfehlers (3.78) bzw. des gewichteten Gleichungsfehlers (3.93) besitzt den Vorteil, dass sowohl das Nebenminimum als auch das globale Minimum gefunden wird (vorausgesetzt, es existiert ein Nebenminimum). Bei der Überwachung müssen beide Lösungen als Kandidaten für den Quellort betrachtet werden, wenn sie sich durch die aktuelle Sensorkonfiguration nicht eindeutig unterscheiden lassen. Um solche Situationen zu vermeiden, sollte der vierte Sensor im Mittelpunkt des Umkreises der anderen drei Sensoren platziert werden (vgl. Beispiel 3.3). Im Fall einer Raumkonfiguration ist der zusätzliche fünfte Sensor entsprechend im Mittelpunkt der Umkugel der anderen vier Sensoren zu platzieren.

\subsection{Zusammenfassung}

In diesem Kapitel wurde ein neues Verfahren zur Quellenlokalisierung bei Diffusion vorgestellt. Es basiert auf einem geometrischen Zugang, bei dem zunächst für jeden Sensor $i$ die Menge $\mathcal{P}_{i}$ bestimmt wird, auf der sich aus der Sicht dieses Sensors die Quelle befinden kann. Durch Betrachten mehrerer $\mathcal{P}_{i}$ lässt sich daraus der Quellort schätzen.

Im Fall von Diffusion bilden diese Mengen für jeden Sensor einen Kreis bzw. eine Kugel um den Sensorstandort. Der Radius, also der Sensor-Quell-Abstand, hängt von der ge- 
messenen Konzentration $C_{i}$ am jeweiligen Sensor sowie der Quellintensität $q_{0}$ und dem Diffusionskoeffizienten $K$ ab. Der Sensor-Quell-Abstand lässt sich in einen skalierbaren Sensor-Quell-Abstand $\varrho_{i}$, der nur von $C_{i}$ abhängt, und einen Skalierungsfaktor $\gamma$, der die Größen $q_{0}$ und $K$ erfasst, zerlegen. $\varrho_{i}$ kann für jeden Sensor sowohl über stationäre als auch instationäre Konzentrationsmessungen ermittelt werden.

Nachdem ein skalierbarer Sensor-Quell-Abstand $\varrho_{i}$ für jeden Sensor geschätzt wurde, muss daraus der globale Skalierungsfaktor $\gamma$ sowie der Quellort $\mathbf{x}_{0}$ bestimmt werden. Im ungestörten Fall führt dies auf konsistente, algebraische Gleichungssysteme. Die allgemeine Lösung für diese Systeme transformiert das Problem in ein unterbestimmtes, lineares Gleichungssystem mit quadratischer Restriktion. Hierfür existiert eine geschlossene Lösung. Ein alternativer, geometrisch interpretierbarer Lösungsweg für den Fall einer Ebenenkonfiguration betrachtet Sensorpaare. Für jedes Sensorpaar wird die Menge aller möglichen Quellorte bestimmt, was auf so genannte Schnittkreise führt. Die Schnittpunkte der Schnittkreise sind Lösungen des Lokalisierungsproblems. Sowohl die allgemeine Lösung als auch die Lösung mittels Schnittkreisen macht deutlich, dass bei Verwenden minimaler Sensoranzahl i. Allg. zwei Lösungen, die wahre und eine scheinbare Lösung, existieren. Es wird gezeigt, dass diese Lösungen gespiegelte Punkte am Umkreis bzw. an der Umkugel der Sensoren sind. Nur durch Hinzunahme eines weiteren Sensors, der nicht auf dem Umkreis bzw. auf der Umkugel liegen darf, ist eine Unterscheidung der beiden Lösungen möglich.

Schließlich wird der gestörte Fall betrachtet. Er führt auf überbestimmte, inkonsistente, algebraische Gleichungssysteme. Diese werden hier im Least-squares-Sinne gelöst. Durch Betrachten des dem geometrischen Kriterium zugeordneten algebraischen Kriteriums gelingt die Transformation in ein lineares Least-squares-Problem mit quadratischer Restriktion. Damit lässt sich ein in [41, 42] vorgestelltes Verfahren nutzen, um eine quasi geschlossene Lösung zu finden. Durch Einführen von Wichtungsfaktoren wird berücksichtigt, dass ein Ausgleich in den gestörten Konzentrationen und nicht in den Sensor-QuellAbständen stattfindet. Damit verringern sich die Abweichungen zwischen wahrem und geschätztem Quellort bei gestörten Konzentrationsmessungen deutlich. De facto verhält sich dann das neue Verfahren aus statistischer Sicht wie das Ausgangsfehlerverfahren (Maximum-likelihood-Schätzung), und das frei von dessen numerischen Problemen. 


\section{Ein neues Verfahren zur Quellenlokalisierung bei Diffusion-Advektion}

Im ersten Schritt des zweistufigen Verfahrens zur modellbasierten Quellenlokalisierung muss - wie im Kapitel 1 beschrieben - für jeden Sensor $i$ die Menge $\mathcal{P}_{i}$ bestimmt werden, auf der sich aus der Sicht von Sensor $i$ die Quelle befinden kann. Für den im vorangegangenen Kapitel untersuchten advektionsfreien Fall (nur Diffusion) stellte die Menge $\mathcal{P}_{i}$ für feste Werte der Quellintensität $q_{0}$ und des Diffusionskoeffizienten $K$ einen Kreis bzw. eine Kugel um den Sensorstandort $\mathbf{x}_{i}$ dar.

Tritt außer der Diffusion auch Advektion auf, die durch ein homogenes Strömungsfeld in positiver $x$-Richtung mit der Strömungsgeschwindigkeit $v_{x}$ hervorgerufen wird, so ergibt die Menge aller Punkte

$$
\mathcal{P}_{i}=\left\{\mathbf{x}_{0} \in \mathbb{R}^{3}:\left\|\mathbf{x}_{i}-\mathbf{x}_{0}\right\|_{2}=d_{i}\left(K, v_{x}, q_{0}, C_{i}, x_{0}\right)\right\}
$$

ein in $x$-Richtung ausgerichtetes $\mathrm{Oval}^{1}$ um den Sensorstandort $\mathbf{x}_{i}$.

Für die Ebenenkonfiguration ist $\mathcal{P}_{i}$ für jeden Sensor $i$ ein zweidimensionales Oval (Abb. 4.1). Unter der Annahme, dass der Diffusionskoeffizient $K$ und die Strömungsgeschwindigkeit $v_{x}$ bekannt sind, ist die Größe des Ovals nur noch von der gemessenen Konzentration $C_{i}$ und von der Quellintensität $q_{0}$ abhängig.

Abbildung 4.1 verdeutlicht, dass sich die Quelle für eine bestimmte Konzentrationsmessung - wie auch beim advektionsfreien Fall - entweder nahe dem Sensorstandort befinden kann, wenn eine kleine Quellintensität vorliegt, oder sich weit weg vom Sensorstandort befinden kann, wenn die Quellintensität groß ist.

\footnotetext{
${ }^{1}$ Unter einem Oval wird hier der Rand einer kompakten, glatten, streng konvexen Menge mit einer Symmetrieachse verstanden. Da die betrachteten Ovale nur eine und nicht zwei Symmetrieachsen besitzen, handelt es sich nicht um Ellipsen.
} 


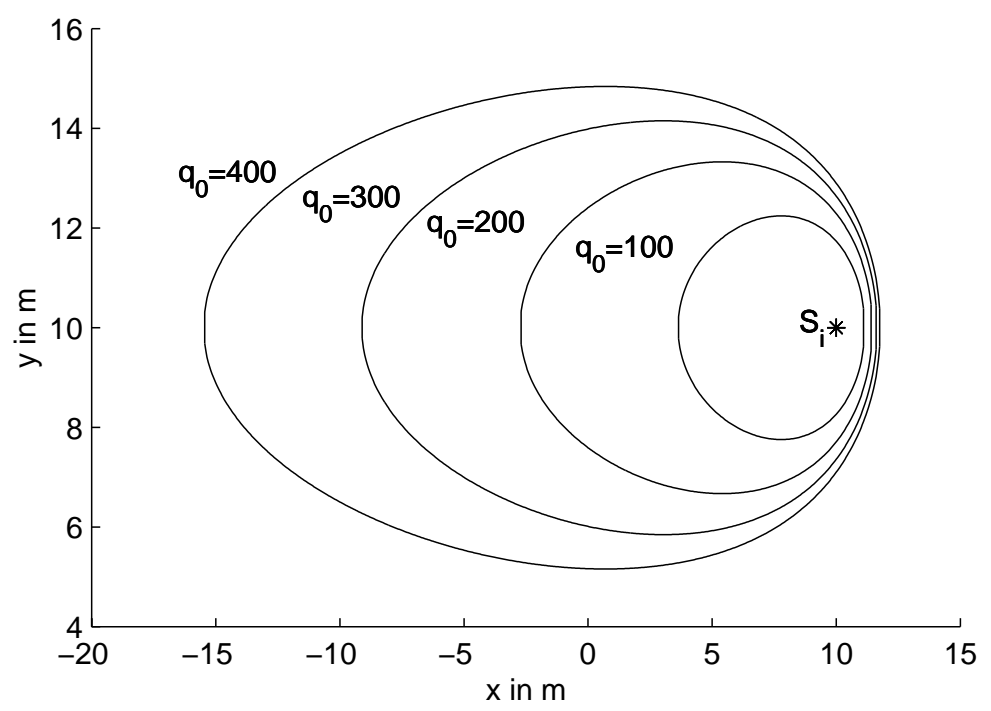

Abb. 4.1: Menge aller möglichen Quellorte $\mathcal{P}_{i}$ aus der Sicht von Sensor $i$ für verschiedene Quellintensitäten $q_{0}$ (in $\mathrm{mg} / \mathrm{s}$ ) bei Diffusion-Advektion.

Zusätzlich bewirkt die Advektion, dass sich die Quelle bei gleicher Quellintensität weiter weg vom Sensorstandort befindet, wenn die Quelle auf der strömungszugewandten Seite liegt bzw. sich näher am Sensorstandort befindet, wenn die Quelle auf der strömungsabgewandten Seite liegt.

Im Fall reiner Diffusion war der Sensor-Quell-Abstand $d_{i}$ für eine bestimmte Konzentration $C_{i}$ und festes $q_{0}$ und $K$ konstant. Damit ergab $\mathcal{P}_{i}$ einen Kreis bzw. eine Kugel. Tritt Advektion in $x$-Richtung auf, so ist der Sensor-Quell-Abstand $d_{i}$ zusätzlich von der $x$ Komponente $x_{0}$ des Quellorts abhängig. Dies führt auf Ovale anstelle von Kreisen bzw. Kugeln. Zudem wirkt nun die Quellintensität $q_{0}$ nicht als linearer Skalierungsfaktor, da $q_{0}$ nichtlinear in $d_{i}$ eingeht. Aus diesem Grund ist hier im Gegensatz zum Diffusionsfall keine Aufspaltung in einen skalierbaren Sensor-Quell-Abstand $\varrho_{i}$ und einen Skalierungsfaktor $\gamma$ möglich. Der Sensor-Quell-Abstand $d_{i}$ ist also bei bekannten Werten von $K, v_{x}$ und $C_{i}$ eine Funktion von $x_{0}$ und $q_{0}$. Diese Größen bewirken eine nichtlineare Skalierung von $d_{i}$. Deshalb wird hier in Analogie zum Diffusionsfall die Größe $d_{i}\left(x_{0}, q_{0}\right)$ als verallgemeinerter skalierbarer Sensor-Quell-Abstand bezeichnet.

Erst durch Betrachten mehrerer Sensoren und damit mehrerer $\mathcal{P}_{i}$ lässt sich der Quellort bestimmen. Im ungestörten Fall ergibt sich bei Wahl der richtigen Quellintensität ein Schnittpunkt aller $\mathcal{P}_{i}$ (Ovale), der den Quellort markiert. Das verdeutlicht Abb. 4.2 für die Ebenenkonfiguration. Im gestörten Fall muss eine Näherungslösung für den Schnittpunkt gefunden werden. 


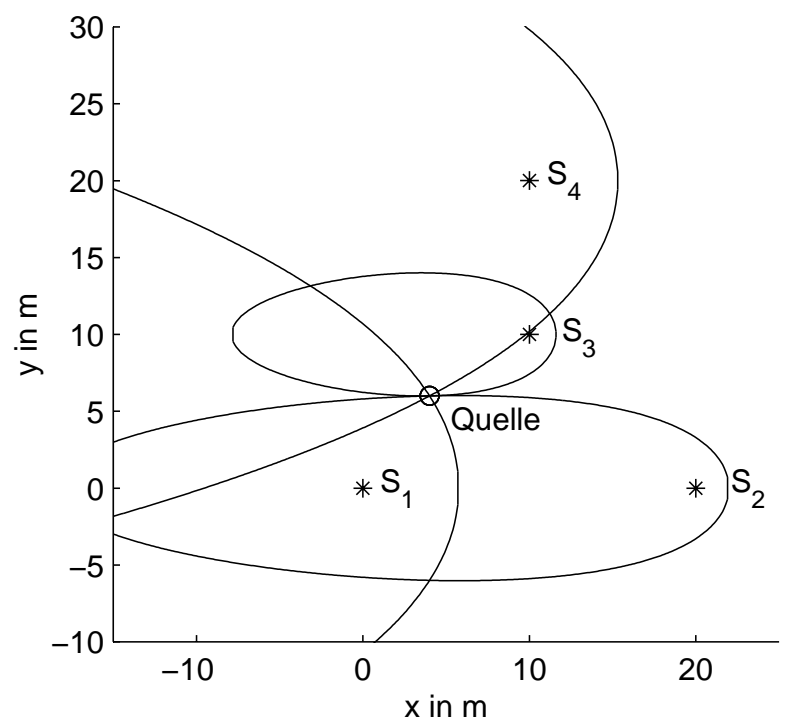

Abb. 4.2: Ovale (Mengen $\left.\mathcal{P}_{i}(i=1, \ldots, 4)\right)$, auf denen sich die Quelle aus Sicht der einzelnen Sensoren $S_{i}$ bei Wahl der wahren Quell- und Ausbreitungsparameter befinden können. Im ungestörten Fall schneiden sich die Ovale im wahren Quellort $\mathbf{x}_{0}$.

\subsection{Schätzung verallgemeinerter skalierbarer Sensor-Quell-Abstände}

Nach (4.1) ist die Menge $\mathcal{P}_{i}$ durch den Sensor-Quell-Abstand $d_{i}\left(K, v_{x}, q_{0}, C_{i}, x_{0}\right)$ bestimmt. Eine explizite Gleichung für $d_{i}$ lässt sich aus der Lösung der Diffusions-AdvektionsGleichung ableiten. Mit der stationären Lösung (2.35) gilt für jeden Sensor

$$
C_{i}=\frac{q_{0} \cdot \exp \left(-\frac{v_{x}}{2 K}\left(d_{i}-\left(x_{i}-x_{0}\right)\right)\right)}{4 \pi K d_{i}}
$$

Dabei bezeichnet $C_{i}$ den stationären Endwert der Konzentration am Sensor $i$. Durch Auflösen nach $d_{i}$ wird

$$
d_{i}\left(K, v_{x}, q_{0}, C_{i}, x_{0}\right)=\frac{2 K}{v_{x}} W_{0}\left(\frac{v_{x} q_{0}}{8 \pi K^{2} C_{i}} \cdot \exp \left(\frac{v_{x}}{2 K} \cdot\left(x_{i}-x_{0}\right)\right)\right)
$$

erhalten. Dabei bezeichnet $W_{0}(\cdot)$ die Lambert-W-Funktion ${ }^{2}$.

Um die Zahl der freien Parameter zu verringern, wird hier angenommen, dass die Strömungsgeschwindigkeit $v_{x}$ und der Diffusionskoeffizient $K$ durch eine A-priori-Identifikation oder durch Online-Messungen bekannt sind. Mit einem Schätzwert $\hat{C}_{i}$ für die stationäre

\footnotetext{
${ }^{2}$ Die Lambert-W-Funktion ist die Inverse der Funktion $f(w)=w \mathrm{e}^{w}=z[20]$.
} 
Konzentration $C_{i}$ (Mittelwert über einige Messwerte) am Sensor $i$ lässt sich der verallgemeinerte Sensor-Quell-Abstand $d_{i}\left(x_{0}, q_{0}\right)$ für jeden Sensor mit

$$
\hat{d}_{i}\left(q_{0}, x_{0}\right)=\frac{2 K}{v_{x}} W_{0}\left(\frac{v_{x} q_{0}}{8 \pi K^{2} \hat{C}_{i}} \cdot \exp \left(\frac{v_{x}}{2 K} \cdot\left(x_{i}-x_{0}\right)\right)\right)
$$

berechnen. Analog zum advektionsfreien Fall (vgl. (3.2)) ergibt sich

$$
\left(x_{i}-x_{0}\right)^{2}+\left(y_{i}-y_{0}\right)^{2}+\left(z_{i}-z_{0}\right)^{2}-\hat{d}_{i}^{2}\left(q_{0}, x_{0}\right)=0 .
$$

Gleichung (4.5) hat die gleiche Struktur wie die implizite Kugelgleichung. Allerdings ist hier der Radius nicht nur eine Funktion der Quellintensität, sondern zugleich abhängig von der $x$-Koordinate der Quelle $x_{0}$. Dadurch wird die Kugel zu einem Oval deformiert.

\subsection{Quellortschätzung im ungestörten Fall}

\subsubsection{Vorbemerkungen}

Um den Quellort zu ermitteln, ist das nichtlineare Gleichungssystem

$$
\hat{d}_{i}\left(x_{0}, q_{0}\right)-\left\|\mathbf{x}_{i}-\mathbf{x}_{0}\right\|_{2}=0 \quad i=1, \ldots, p
$$

zu lösen.

In diesem Abschnitt wird zunächst der ungestörte Fall behandelt. Die geschätzten SensorQuell-Abstände sind gleich den wahren Werten; anstelle von $\hat{d}_{i}$ wird $d_{i}$ benutzt. Die Untersuchungen gliedern sich nach den Sensor-Quell-Konfigurationen (vgl. Anschnitt 3.2.1):

- Linienkonfiguration,

- Ebenenkonfiguration,

- Raumkonfiguration und

- Halbraumkonfiguration.

Wie auch im Fall reiner Diffusion wird für den ungestörten Fall versucht, den Quellort mit minimaler Sensoranzahl $p=m$ zu ermitteln. Für die Berechnung des Quellorts konnten bei reiner Diffusion geschlossene Lösungen gefunden werden. Das gelingt im Fall von Diffusion-Advektion leider nicht. Stattdessen lässt sich der Quellort nur iterativ bestimmen. Dennoch erlauben die Untersuchungen Aussagen zur Existenz und Eindeutigkeit von Lösungen sowie zur geeigneten Sensorplatzierung. In Abschnitt 4.4 werden die gewonnenen Ergebnisse auf den gestörten Fall erweitert. 


\subsubsection{Linienkonfiguration}

Die Linienkonfiguration erfordert für die Ermittlung der unbekannten Größen $x_{0}$ und $q_{0}$ mindestens $m=2$ Sensoren an den Standorten $x_{1}$ und $x_{2}\left(x_{1}<x_{2}\right)$. Die Sensor-QuellAbstände sind für ein bestimmtes $q_{0}$ und $x_{0}$ durch

$$
d_{i}\left(x_{0}, q_{0}\right)=\left|x_{i}-x_{0}\right|
$$

gegeben. Für den Quellort lautet das zu lösende Gleichungssystem

$$
\begin{aligned}
& d_{1}\left(x_{0}, q_{0}\right)-\left|x_{1}-x_{0}\right|=0 \\
& d_{2}\left(x_{0}, q_{0}\right)-\left|x_{2}-x_{0}\right|=0 .
\end{aligned}
$$

Per Iteration können damit die Parameter $x_{0}$ und $q_{0}$ gefunden werden. Ein besserer $\mathrm{Zu}-$ gang lässt sich finden, wenn berücksichtigt wird, dass stets eine Lösung $x_{0, I}$ existiert, die zwischen $x_{1}$ und $x_{2}$ liegt, für die also

$$
x_{1}<x_{0, I}<x_{2}
$$

gilt, auch wenn sich die wahre Quelle außerhalb dieses Intervalls befindet. Damit vereinfacht sich (4.8) zu

$$
\begin{aligned}
& d_{1}\left(x_{0}, q_{0}\right)-\left(x_{0}-x_{1}\right)=0=: J_{1} \\
& d_{2}\left(x_{0}, q_{0}\right)-\left(x_{2}-x_{0}\right)=0=: J_{2} .
\end{aligned}
$$

Die iterative Suche muss für $x_{0}$ nur noch im Intervall $x_{1}<x_{0}<x_{2}$ erfolgen.

Beispiel 4.1 Im Fall einer Linienkonfiguration mit $x_{1}=0 \mathrm{~m}, x_{2}=10 \mathrm{~m}$ und einer Quelle bei $x_{0}=2 \mathrm{~m}$ werden im ungestörten Fall die stationären Konzentrationen $C_{1}=1.98 \mathrm{mg} / \mathrm{m}^{3}$ und $C_{2}=9.95 \mathrm{mg} / \mathrm{m}^{3}$ gemessen ( $\left.q_{0}=100 \mathrm{mg} / \mathrm{s}, v_{x}=0.3 \mathrm{~m} / \mathrm{s}, K=0.2 \mathrm{~m}^{2} / \mathrm{s}\right)$. Abbildung 4.3 (links) zeigt das stationäre Konzentrationsprofil. Abbildung 4.3 (rechts) zeigt die Gleichungsfehler $J_{1}$ und $J_{2}$ gemäß (4.10) in Abhängigkeit von $x$ für den wahren Wert von $q_{0}$.

Die Verläufe von $J_{1}$ und $J_{2}$ in Abb. 4.3 lassen eine schnelle Konvergenz iterativer Verfahren beim Lösen von (4.10) erwarten.

Wenn der Quellort $x_{0, I}$ gefunden ist, der zwischen $x_{1}$ und $x_{2}$ liegt, muss geprüft werden, ob nicht noch ein anderer Quellort $x_{0, I I}$ außerhalb von $\left[x_{1}, x_{2}\right]$ gleiche Konzentrationswerte an den Sensorstandorten hervorrufen kann. Dass solche Lösungen existieren, zeigt Abb. 4.4. Bei $C_{1}>C_{2}$ kann eine zweite (scheinbare) Lösung links von $x_{1}$ liegen (Abb. 4.4 links). 

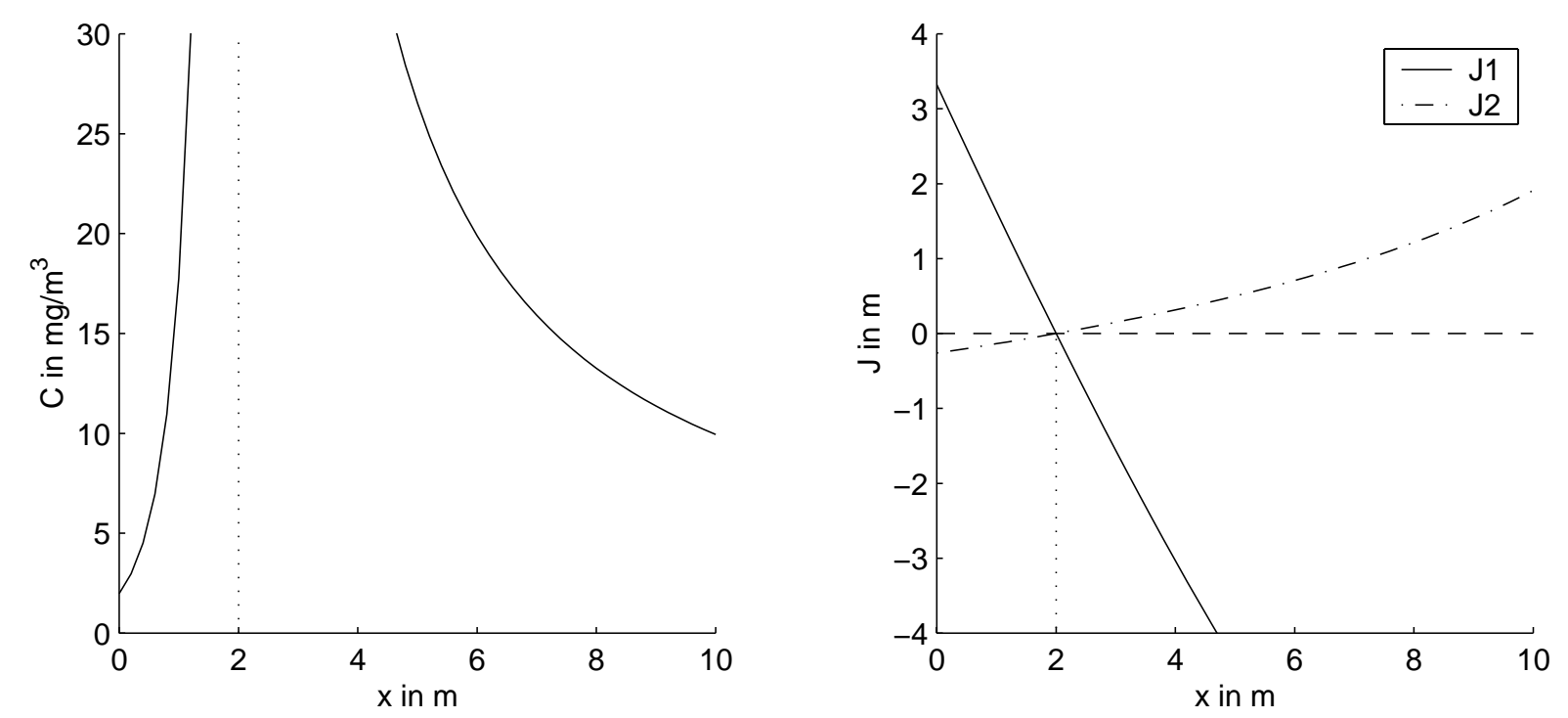

Abb. 4.3: Links: Konzentrationsprofil bei eindimensionaler Sensor-Quell-Anordnung. Rechts: Gleichungsfehler $J_{1}$ und $J_{2}$ in Abhängigkeit von $x$. Beim gesuchten Wert $x_{0}=2 \mathrm{~m}$ ist $J_{1}=$ $J_{2}=0 \mathrm{~m}$.
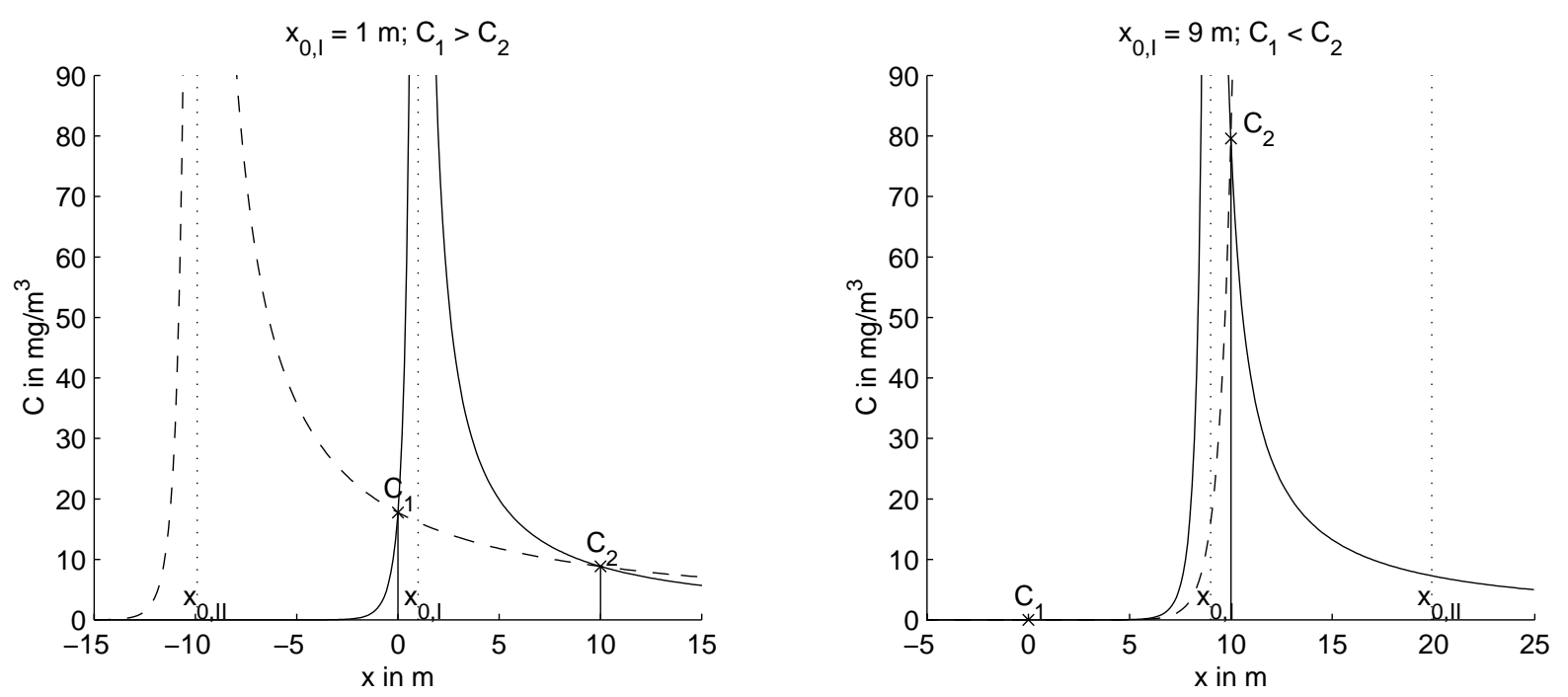

Abb. 4.4: Zusätzliche Lösungen $x_{0, I I}$ für den Quellort außerhalb von $\left[x_{1}, x_{2}\right]$ für den Fall $C_{1}>C_{2}$ (links) und $C_{1}<C_{2}$ (rechts). Die Konzentrationsprofile für die wahren Quellorte $x_{0, I}$ (durchgängige Linien) schneiden sich mit den Konzentrationsprofilen der scheinbaren Quellorte $x_{0, I I}$ (gestrichelte Linien) in den Sensorstandorten $x_{1}$ und $x_{2}$.

Andernfalls kann sich eine zweite Lösung rechts von $x_{2}$ befinden (Abb. 4.4 rechts). Ob die zweite Lösung überhaupt existiert und ob sie links oder rechts des Intervalls $\left[x_{1}, x_{2}\right]$ liegt, hängt also von $C_{1}$ und $C_{2}$ ab. Dies wird nachfolgend eingehender untersucht.

Im Fall reiner Diffusion liegt unabhängig vom Diffusionskoeffizienten die scheinbare Lösung stets bei $x_{0, I I}<x_{1}$ für $C_{1}>C_{2}$ sowie bei $x_{0, I I}>x_{2}$ für $C_{1}<C_{2}$ (vgl. Abschnitt 3.2.2). Im Grenzfall $C_{1}=C_{2}$ befand sich der wahre Quellort in der Mitte $x_{M}$ zwischen den 
Sensoren $\left(x_{0, I}=\left(x_{2}-x_{1}\right) / 2+x_{1}=x_{M}\right)$, und ein scheinbarer Quellort existierte nur im Unendlichen $\left(x_{0, I I}= \pm \infty\right)$. Eine Entscheidung über die Lage der scheinbaren Lösungen lässt sich damit also auch darüber treffen, ob für den wahren Quellort $x_{0, I}<x_{M}$ oder $x_{0, I}>x_{M}$ gilt.

$x_{M}$ kann auch als derjenige Punkt definiert werden, in dem eine Quelle in allen Sensoren die gleichen Konzentrationen hervorruft. Im Fall der Ebenen- bzw. Raumkonfiguration bei Diffusion war das der Mittelpunkt $\mathbf{x}_{M}$ des Umkreises bzw. der Umkugel.

Im Fall von Diffusion-Advektion ist die Lage eines solchen Punktes $x_{M}$ vom Diffusionskoeffizienten $K$ und von der Strömungsgeschwindigkeit $v_{x}$ abhängig. $x_{M}$ lässt sich nicht analytisch bestimmen, sondern nur als Quellort für den Fall $C_{1}=C_{2}$ iterativ ermitteln.

Eine implizite Darstellung für $x_{M}$ wird durch Dividieren der Gleichungen (4.2) für Sensor 1 und 2 unter Berücksichtigung von $x_{1}<x_{0}<x_{2}$ und damit $d_{1}=x_{0}-x_{1}$ sowie $d_{2}=x_{2}-x_{1}$

$$
\frac{C_{1}}{C_{2}}=\frac{x_{2}-x_{0}}{x_{0}-x_{1}} \cdot \exp \left(\frac{-v_{x}}{K}\left(x_{0}-x_{1}\right)\right)
$$

und nachfolgende Substitution $x_{0}:=x_{M}$ sowie $C_{1} / C_{2}:=1$

$$
1=\frac{x_{2}-x_{M}}{x_{M}-x_{1}} \cdot \exp \left(\frac{-v_{x}}{K}\left(x_{M}-x_{1}\right)\right)
$$

erhalten.

Existiert ein scheinbarer Quellort $x_{0, I I}$, dann ruft er an den Sensorstandorten $x_{1}$ und $x_{2}$ die gleichen Konzentrationen $C_{1}$ und $C_{2}$ hervor wie der wahre Quellort $x_{0, I}$. Daraus folgt das Gleichungssystem

$$
\begin{aligned}
& C_{1}\left(x_{0}=x_{0, I}\right)=C_{1}\left(x_{0}=x_{0, I I}\right) \\
& C_{2}\left(x_{0}=x_{0, I}\right)=C_{2}\left(x_{0}=x_{0, I I}\right) .
\end{aligned}
$$

Für das Lösen muss zwischen den Fällen $C_{1} \geq C_{2}\left(x_{0, I I}<x_{1}<x_{0, I}<x_{2}\right)$ und $C_{1}<C_{2}$ $\left(x_{1}<x_{0, I}<x_{2}<x_{0, I I}\right)$ unterschieden werden.

Fall $C_{1} \geq C_{2}$

Mit (4.2) und $x_{0, I I}<x_{1}<x_{0, I}<x_{2}$ wird aus (4.13)

$$
\begin{aligned}
\frac{1}{x_{0, I}-x_{1}} \cdot \exp \left(\frac{-v_{x}}{K} \cdot\left(x_{0, I}-x_{1}\right)\right) & =\frac{1}{x_{1}-x_{0, I I}} \\
\frac{1}{x_{2}-x_{0, I}} & =\frac{1}{x_{2}-x_{0, I I}}
\end{aligned}
$$


erhalten. Dividieren der Gleichungen liefert

$$
\frac{x_{2}-x_{0, I}}{x_{0, I}-x_{1}} \cdot \exp \left(\frac{-v_{x}}{K} \cdot\left(x_{0, I}-x_{1}\right)\right)=\frac{x_{2}-x_{0, I I}}{x_{1}-x_{0, I I}} .
$$

Die linke Seite wird zu

$$
\kappa_{l}:=\frac{x_{2}-x_{0, I}}{x_{0, I}-x_{1}} \cdot \exp \left(\frac{-v_{x}}{K} \cdot\left(x_{0, I}-x_{1}\right)\right)
$$

zusammengefasst. Durch die rechte Seite entsteht mit $x_{0, I I}<x_{1}<x_{0, I}<x_{2}$ die Forderung $\kappa_{l} \geq 1$. Im hier betrachteten Fall $C_{1} \geq C_{2}$, also $x_{0, I} \leq x_{M}$, wird diese Forderung wegen (4.12) stets erfüllt. Für die Lage des scheinbaren Quellorts $x_{0, I I}$ folgt

$$
x_{0, I I}=\frac{\kappa_{l} x_{1}-x_{2}}{\kappa_{l}-1} .
$$

Fall $C_{1}<C_{2}$

Mit (4.2) und $x_{1}<x_{0, I}<x_{2}<x_{0, I I}$ wird aus (4.13)

$$
\begin{aligned}
\frac{1}{x_{0, I}-x_{1}} \cdot \exp \left(\frac{-v_{x}}{K}\left(x_{0, I}-x_{1}\right)\right) & =\frac{1}{x_{0, I I}-x_{1}} \cdot \exp \left(\frac{-v_{x}}{K}\left(x_{0, I I}-x_{1}\right)\right) \\
\frac{1}{x_{2}-x_{0, I}} & =\frac{1}{x_{0, I I}-x_{2}} \cdot \exp \left(\frac{-v_{x}}{K}\left(x_{0, I I}-x_{1}\right)\right) .
\end{aligned}
$$

Dividieren der Gleichungen und Umstellen liefert

$$
\frac{x_{2}-x_{0, I}}{x_{0, I}-x_{1}} \cdot \exp \left(\frac{-v_{x}}{K}\left(x_{0, I}-x_{2}\right)\right)=\frac{x_{0, I I}-x_{2}}{x_{0, I I}-x_{1}} .
$$

Die linke Seite wird zu

$$
\kappa_{r}:=\frac{x_{2}-x_{0, I}}{x_{0, I}-x_{1}} \cdot \exp \left(\frac{-v_{x}}{K}\left(x_{0, I}-x_{2}\right)\right)
$$

zusammengefasst. Durch die rechte Seite entsteht mit $x_{1}<x_{0, I}<x_{2}<x_{0, I I}$ die Forderung $0<\kappa_{r}<1$. Der kritische Fall $\kappa_{r}=1$ wird bei $x_{0, I}=x_{2}-\left(x_{M}-x_{1}\right)$ erreicht. Dies lässt sich zeigen, indem in (4.20) $x_{0, I}:=x_{2}-\left(x_{M}-x_{1}\right)$ substituiert wird. Dies führt auf

$$
\kappa_{r}=\frac{x_{2}-x_{M}}{x_{M}-x_{1}} \cdot \exp \left(\frac{-v_{x}}{K}\left(x_{M}-x_{1}\right)\right) .
$$

Da die rechte Seite identisch mit (4.12) ist, gilt $\kappa_{r}=1$ für $x_{0, I}=x_{2}-\left(x_{M}-x_{1}\right)$. Für $0<\kappa_{r}<1$ lässt sich der zweite Quellort mit

$$
x_{0, I I}=\frac{\kappa_{r} x_{1}-x_{2}}{\kappa_{r}-1}
$$

berechnen. 
Zusammenfassend verdeutlicht Abb. 4.5 die Bereiche, die zwischen $x_{1}$ und $x_{2}$ existieren:

- Zu einer Quelle bei $x_{1} \leq x_{0, I} \leq x_{M}$ existiert ein zweiter möglicher Quellort $x_{0, I I}<x_{1}$ (4.17). Für den Spezialfall $C_{1}=C_{2}$, also $x_{0, I}=x_{M}$, ist $x_{0, I I}=-\infty$.

- Zu einer Quelle bei $x_{M}<x_{0, I} \leq\left(x_{2}-\left(x_{M}-x_{1}\right)\right)$ existiert kein weiterer möglicher Quellort.

- $\mathrm{Zu}$ einer Quelle bei $\left(x_{2}-\left(x_{M}-x_{1}\right)<x_{0, I}<x_{2}\right.$ existiert ein zweiter möglicher Quellort $x_{0, I I}>x_{2}(4.22)$.

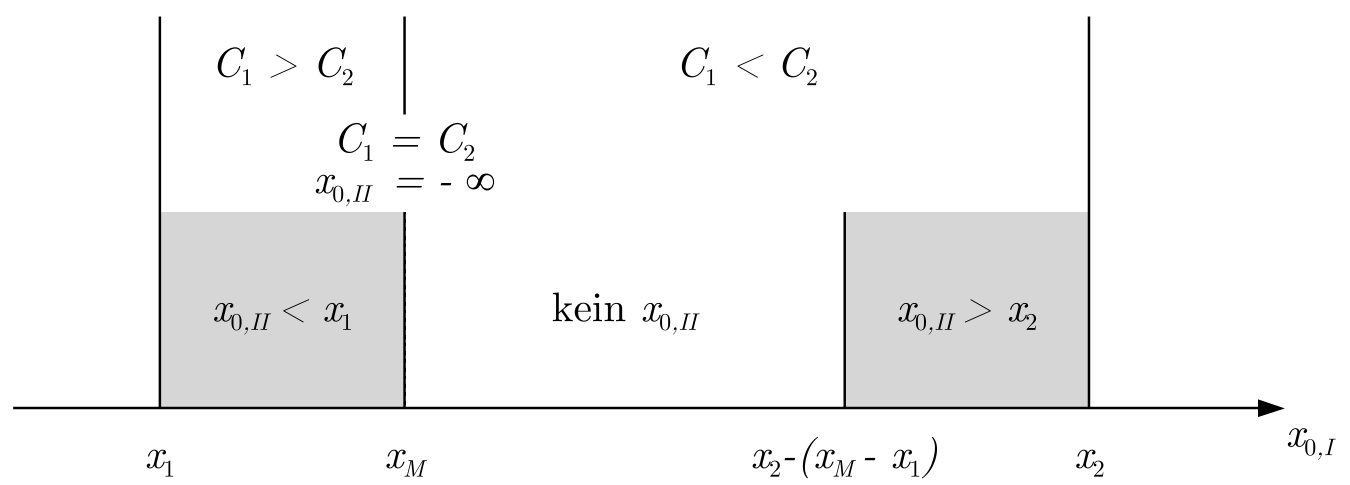

Abb. 4.5: Übersicht über die Lage eines zweiten möglichen Quellorts $x_{0, I I}$ in Abhängigkeit von der Lage des Quellorts $x_{0, I}$.

Bei sinkender Strömungsgeschwindigkeit $v_{x}$ schrumpft der mittlere Bereich, bis er im Fall reiner Diffusion $\left(v_{x}=0\right)$ komplett verschwindet. Bei reiner Diffusion existiert damit, wie im vorangegangenen Kapitel gezeigt, stets ein zweiter Quellort $x_{0, I I}$, der die gleichen Konzentrationen $C_{1}, C_{2}$ hervorrufen kann wie $x_{0, I}$. Bei Diffusion-Advektion ist dies nicht immer der Fall.

\subsubsection{Ebenenkonfiguration}

\section{Iterative Quellortbestimmung}

Bei der Ebenenkonfiguration sind mindestens $m=3$ Sensoren notwendig, um den Quellort $\mathbf{x}_{0}=\left(x_{0}, y_{0}\right)^{T}$ und $q_{0}$ zu bestimmen. Das sich damit ergebende Gleichungssystem

$$
\begin{aligned}
& d_{1}\left(x_{0}, q_{0}\right)-\left\|\mathbf{x}_{1}-\mathbf{x}_{0}\right\|_{2}=0 \\
& d_{2}\left(x_{0}, q_{0}\right)-\left\|\mathbf{x}_{2}-\mathbf{x}_{0}\right\|_{2}=0 \\
& d_{3}\left(x_{0}, q_{0}\right)-\left\|\mathbf{x}_{3}-\mathbf{x}_{0}\right\|_{2}=0
\end{aligned}
$$

lässt sich iterativ lösen. 
Wie auch im Fall der Linienkonfiguration können bei Verwenden von drei Sensoren zwei mögliche Lösungen existieren. Bei der Optimierung wird je nach Wahl der Startwerte eine der beiden Lösungen gefunden. Nachfolgend wird eine geometrische Interpretation der Entstehung der beiden Lösungen gegeben. Dank des damit gewonnenen geometrischen Verständnisses können gezielt Startwerte für die Iteration vorgegeben werden, um eine eventuell existierende zweite Lösung zu finden. Um ein eindeutiges Minimum und damit eine eindeutige Lösung zu erhalten, sind vier Sensoren notwendig.

\section{Geometrische Interpretation mittels Schnittkurven}

Eine geometrische Interpretation über Schnittkurven (vgl. Abschnitt 3.2.3) ermöglicht Aussagen über die Existenz und Vielfalt von Lösungen des Quellenlokalisierungsproblems.

Die Idee ist wiederum, für jeweils ein Paar von Sensoren die Menge aller Punkte (Schnittkurve) zu bestimmen, auf denen sich aus der Sicht dieser beiden Sensoren die Quelle befinden kann. Im Fall reiner Diffusion war die Schnittkurve für ein Sensorpaar ein so genannter Schnittkreis, dessen Parameter (Mittelpunkt und Radius) analytisch ermittelt werden konnten. Bei den drei minimal notwendigen Sensoren ergaben sich zwei Schnittkreise, die sich in dem möglichen Quellort bzw. den möglichen Quellorten schnitten.

Im Advektionsfall formen die Schnittkurven keine Kreise. Auch sind die Parameter der Schnittkurven nicht analytisch bestimmbar. Dennoch lassen sich die Schnittkurven problemlos numerisch generieren.

Dazu wird für jedes $q_{0}$ und jeden Sensor $i$ das zugehörige Oval $\mathcal{P}_{i}$ erzeugt, indem für jedes $x_{0}$ die zugehörigen $y_{0}$ mit

$$
y_{0}=y_{i} \pm \sqrt{d_{i}^{2}\left(x_{0}, q_{0}\right)-\left(x_{i}-x_{0}\right)^{2}}
$$

berechnet werden. Anschließend werden die paarweisen Schnittpunkte der Ovale für jedes $q_{0}$ bestimmt. Nachfolgend werden die Schnittlinien für die Sensorpaare 1,2 und 1,3 berücksichtigt. Da jede Schnittlinie mit einer Gleichung des zu

$$
\begin{aligned}
& d_{1}\left(x_{0}, q_{0}\right)-\left\|\mathbf{x}_{1}-\mathbf{x}_{0}\right\|_{2}=d_{2}\left(x_{0}, q_{0}\right)-\left\|\mathbf{x}_{2}-\mathbf{x}_{0}\right\|_{2} \\
& d_{1}\left(x_{0}, q_{0}\right)-\left\|\mathbf{x}_{1}-\mathbf{x}_{0}\right\|_{2}=d_{3}\left(x_{0}, q_{0}\right)-\left\|\mathbf{x}_{3}-\mathbf{x}_{0}\right\|_{2}
\end{aligned}
$$

umgeformten Gleichungssystems (4.23) korrespondiert, wird klar, dass im ungestörten Fall (konsistentes Gleichungssystem) eine weitere Gleichung

$$
d_{2}\left(x_{0}, q_{0}\right)-\left\|\mathbf{x}_{2}-\mathbf{x}_{0}\right\|_{2}=d_{3}\left(x_{0}, q_{0}\right)-\left\|\mathbf{x}_{3}-\mathbf{x}_{0}\right\|_{2}
$$

und damit das Sensorpaar 2,3 keine weitere Information liefert. 
Beispiel 4.2 Für die Ebenenkonfiguration mit den Sensoren bei $\mathbf{x}_{1}=(-1.2 \mathrm{~m},-0.6 \mathrm{~m})^{T}$, $\mathbf{x}_{2}=(6.7 \mathrm{~m},-2.1 \mathrm{~m})^{T}$ und $\mathbf{x}_{3}=(3.7 \mathrm{~m}, 2.7 \mathrm{~m})^{T}$ mit einer Quelle bei $\mathbf{x}_{0, I}=(-0.5 \mathrm{~m}, 0 \mathrm{~m})^{T}$ ergeben sich die in Abb. 4.6 dargestellten Schnittkurven für die Sensorpaare 1, 2 und 1,3.

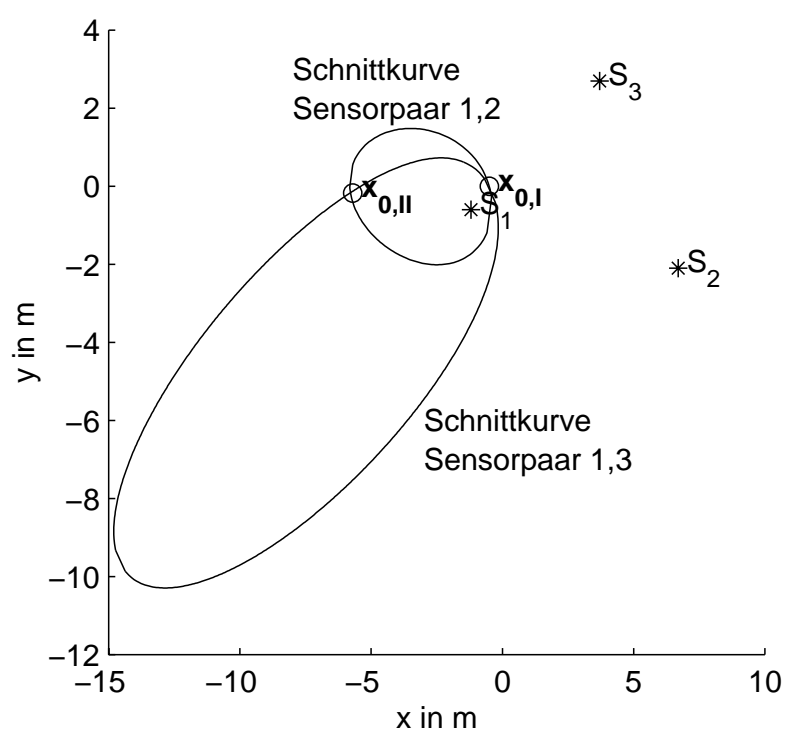

Abb. 4.6: Schnittkurven für Sensorpaare 1,2 und 1,3 sowie wahrer und scheinbarer Quellort als Schnittpunkte der Schnittkurven bei Diffusion-Advektion.

Anhand von Abbildung 4.6 kann vermutet werden, dass die Schnittkurven im Fall von Diffusion-Advektion Ellipsen sind. Wird die erste Gleichung in (4.25) zu

$$
\begin{array}{r}
\left(d_{2}^{2}\left(x_{0}, q_{0}\right)-d_{1}^{2}\left(x_{0}, q_{0}\right)\right) \mathbf{x}_{0}^{T} \mathbf{x}_{0}-2\left(d_{2}^{2}\left(x_{0}, q_{0}\right) \mathbf{x}_{1}^{T}-d_{1}^{2}\left(x_{0}, q_{0}\right) \mathbf{x}_{2}^{T}\right) \mathbf{x}_{0} \\
+d_{2}^{2}\left(x_{0}, q_{0}\right) \mathbf{x}_{1}^{T} \mathbf{x}_{1}-d_{1}^{2}\left(x_{0}, q_{0}\right) \mathbf{x}_{2}^{T} \mathbf{x}_{2}=0
\end{array}
$$

umgeformt, ist zu erkennen, dass der Faktor $\left(d_{2}^{2}\left(x_{0}, q_{0}\right)-d_{1}^{2}\left(x_{0}, q_{0}\right)\right)$ auf die $x$ - und $y$ Komponente des quadratischen Anteils der Schnittkurve gleichermaßen wirkt. Ist dieser Faktor konstant, werden Kreise (Grenzfall Diffusion) aber niemals echte Ellipsen als Schnittkurven erhalten. Da aber $d_{1}$ und $d_{2}$ nichtlinear von $x_{0}$ und $q_{0}$ abhängen (Gl. (4.3)), sind die Schnittkurven im Allgemeinen keine Kreise, sondern analytisch nicht beschreibbare Kurven.

Aus Abbildung 4.6 ist weiterhin zu erkennen, dass bei Verwendung von drei Sensoren im Fall einer Ebenenkonfiguration neben dem wahren Quellort $\mathbf{x}_{0, I}$ noch ein weiterer scheinbarer Quellort $\mathbf{x}_{0, I I} \approx(-5.7 \mathrm{~m},-0.18 \mathrm{~m})^{T}$ existieren kann. Aber wie auch schon bei der Linienkonfiguration gibt es bei der Ebenenkonfiguration Quellorte, zu denen kein zweiter möglicher Quellort existiert. Das verdeutlicht Abb. 4.7 für die in Beispiel 4.2 
verwendete Sensorkonfiguration und einen Quellort $\mathbf{x}_{0, I}=(0.5 \mathrm{~m}, 0 \mathrm{~m})^{T}$. Die Schnittkurven schneiden sich nur in einem Punkt, nämlich dem wahren Quellort.

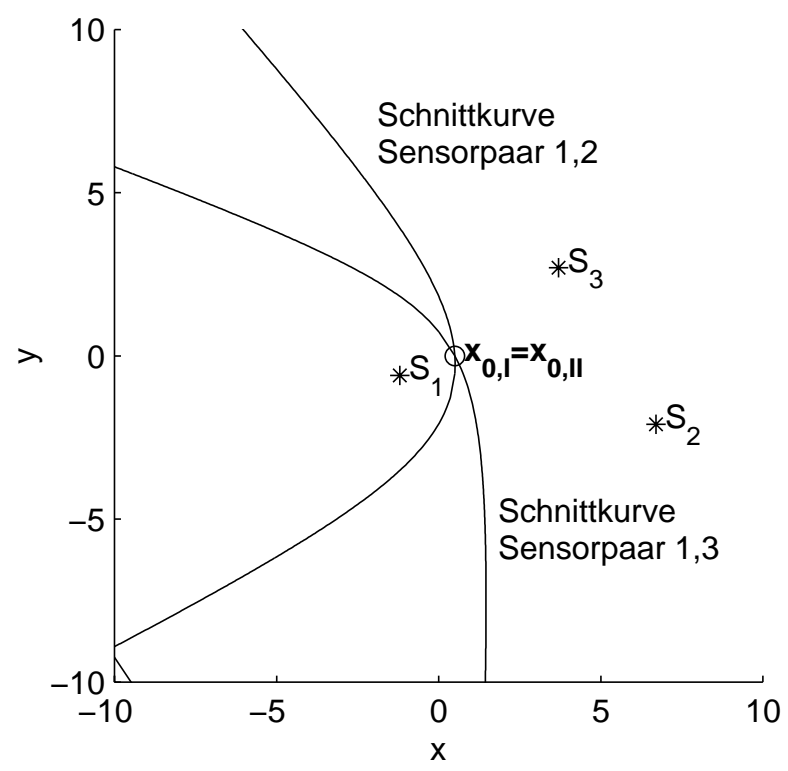

Abb. 4.7: Schnittkurven für die Sensorpaare 1,2 und 1, 3 sowie Quellort als einziger Schnittpunkt der Schnittkurven bei Diffusion-Advektion.

\subsubsection{Raumkonfiguration}

Bei der Raumkonfiguration sind mindestens $m=4$ Sensoren notwendig, um den Quellort $\mathbf{x}_{0}=\left(x_{0}, y_{0}, z_{0}\right)^{T}$ und die Quellintensität $q_{0}$ zu ermitteln. Wie auch bei der Linien- und Ebenenkonfiguration lässt sich der Quellort durch iteratives Lösen von

$$
\begin{aligned}
& d_{1}\left(x_{0}, q_{0}\right)-\left\|\mathbf{x}_{1}-\mathbf{x}_{0}\right\|_{2}=0 \\
& d_{2}\left(x_{0}, q_{0}\right)-\left\|\mathbf{x}_{2}-\mathbf{x}_{0}\right\|_{2}=0 \\
& d_{3}\left(x_{0}, q_{0}\right)-\left\|\mathbf{x}_{3}-\mathbf{x}_{0}\right\|_{2}=0 \\
& d_{4}\left(x_{0}, q_{0}\right)-\left\|\mathbf{x}_{4}-\mathbf{x}_{0}\right\|_{2}=0
\end{aligned}
$$

finden.

Eine Analyse weiterer Lösungen für den Quellort ist im Fall einer Raumkonfiguration auf der Basis von Schnittkurven schwierig. Jedoch erlaubt eine Analogiebetrachtung zum Diffusionsfall und zu anderen Sensor-Quell-Konfigurationen qualitative Aussagen.

Im Fall von reiner Diffusion existierten im ungestörten Fall mindestens eine Lösung, i. Allg. sogar zwei Lösungen für den Quellort. Dies galt für die Linien- und Ebenen- als auch für die Raum- und Halbraumkonfiguration. Im Fall von Diffusion-Advektion wurde für die 
Linien- und Ebenenkonfiguration gezeigt, dass entweder eine Lösung oder zwei Lösungen existieren. Dies lässt den Schluss zu, dass auch bei der Raum- und Halbraumkonfiguration entweder eine Lösung oder zwei Lösungen auftreten. Eine Unterscheidung zwischen dem wahren und einem möglichen scheinbaren Quellort ist in jedem Fall durch einen geeignet angeordneten zusätzlichen Sensor möglich (siehe Abschnitt 4.3.2).

\subsubsection{Halbraumkonfiguration}

Bei der Halbraumkonfiguration sind für die Lokalisierung des Quellorts $\mathbf{x}_{0}=\left(x_{0}, y_{0}\right)^{T}$ mindestens $m=3$ Sensoren notwendig. Die Lösung erfolgt wie im Fall der Ebenenkonfiguration.

\subsubsection{Allgemeine Lösung}

Die allgemeine Lösung für die Quellenlokalisierung auf der Basis minimaler Sensoranzahl $m$ wird als Lösung von

$$
d_{i}\left(x_{0}, q_{0}\right)-\left\|\mathbf{x}_{i}-\mathbf{x}_{0}\right\|_{2}=0 ; \quad i=1, \ldots, m
$$

erhalten. Zur Lösung lassen sich Gradientenverfahren verwenden; eine spezielle Lösung wie im Fall von Diffusion lässt sich nicht angeben.

\subsection{Untersuchung der Lösung}

\subsubsection{Eigenschaften von wahrem und scheinbarem Quellort}

Der vorangegangene Abschnitt zeigte, dass im ungestörten Fall bei Verwendung der minimalen Anzahl von Sensoren zwei Lösungen für den Quellort existieren können. Für den Fall reiner Diffusion konnte gezeigt werden, dass die beiden Quellorte gespiegelte Punkte am Umkreis bzw. an der Umkugel der Sensoren sind. Im Fall von Diffusion-Advektion sind solche quantitativen Aussagen über die Lage der Lösungen nicht möglich. Einzig für die Linienkonfiguration konnte eine geschlossene Lösung für die Lage des scheinbaren Quellorts auf der Basis der Lage des wahren Quellorts angegeben werden. Nachfolgend werden für den Advektionsfall am Beispiel der Ebenenkonfiguration qualitative Aussagen über die Eigenschaften der wahren und der scheinbaren Lösung erarbeitet, die sich später zur Sensorplatzierung nutzen lassen. 
Abbildung 4.8 zeigt die Konzentrationsprofile, die sich für den wahren Quellort $\mathbf{x}_{0, I}$ und für den scheinbaren Quellort $\mathbf{x}_{0, I I}$ gemäß Beispiel 4.2 ergeben. In der $(x, y)$-Ebene sind die Punkte eingezeichnet, über denen sich beide Konzentrationsprofile schneiden (Konzentrationsschnittlinie). Im Fall reiner Diffusion ergaben diese Punkte genau den Umkreis der Sensoren. Hier im Advektionsfall bilden die Punkte eine geschlossen Kurve (aber keinen Kreis), auf der alle Sensoren liegen.

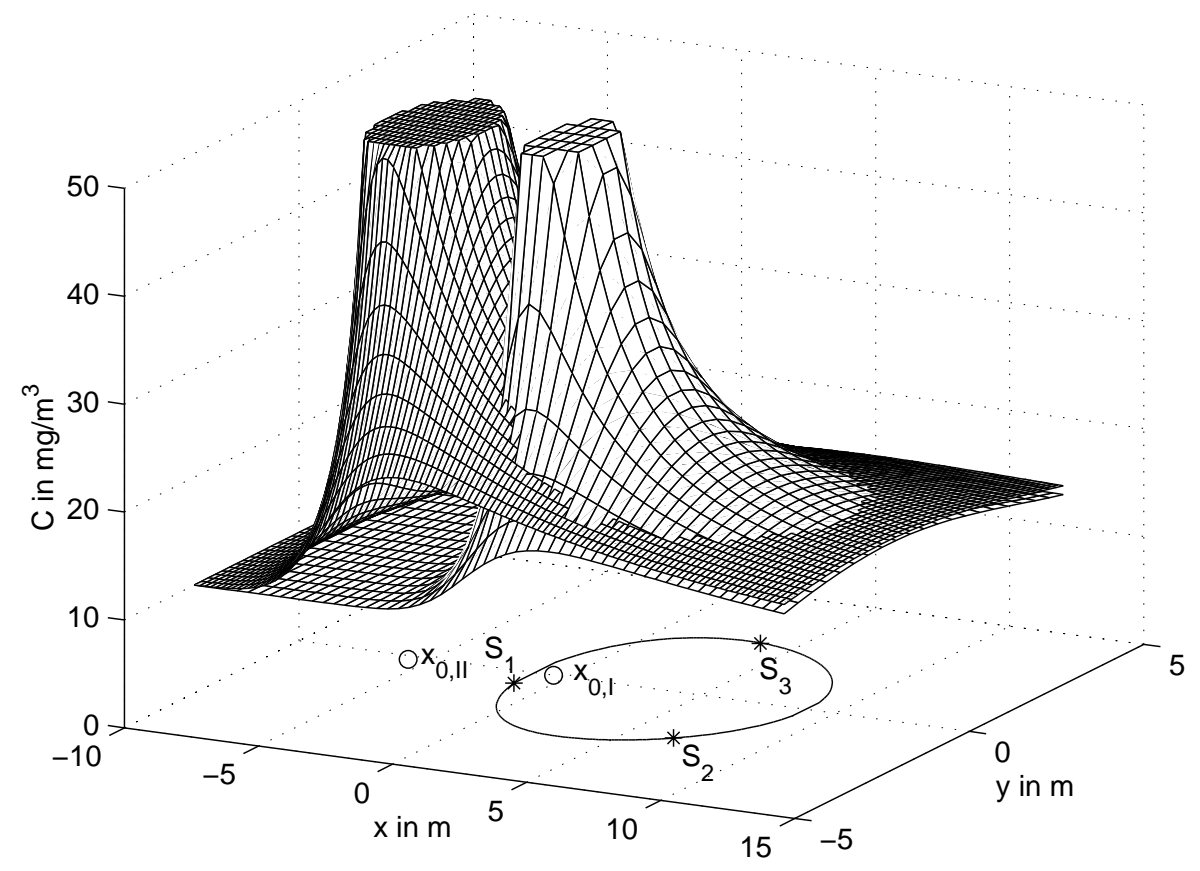

Abb. 4.8: Konzentrationsprofile für wahren und scheinbaren Quellort $\mathbf{x}_{0, I}$ bzw. $\mathbf{x}_{0, I I}$ gemäß Beispiel 4.2 sowie in die $(x, y)$-Ebene projizierte Konzentrationsschnittlinie.

Während im Diffusionsfall die projizierte Konzentrationsschnittlinie unabhängig vom Quellort stets den Umkreis der Sensoren bildet, ist die Form und Lage dieser Schnittlinie im Advektionsfall abhängig vom Quellort. Das verdeutlicht Abb. 4.9. Für vier verschiedene Quellorte $\mathbf{x}_{0, I}$ sind die projizierten Konzentrationsschnittlinien dargestellt. Allen Schnittlinien ist gemeinsam, dass sie die Sensorstandorte schneiden und bezüglich dieser Standorte konvexe Mengen ${ }^{3}$ umranden.

\subsubsection{Konsequenzen für die Sensorplatzierung}

Wie im Abschnitt 4.2 herausgearbeitet wurde, ist eine Unterscheidung zwischen wahrem und scheinbarem Quellort nur mit einem weiteren Sensor möglich.

\footnotetext{
${ }^{3}$ Eine Menge ist konvex, wenn sie mit je zwei Elementen $x$ und $y$ die gesamte Verbindungsstrecke $\{\lambda x+(1-\lambda) y: 0 \leq \lambda \leq 1\}$ zwischen $x$ und $y$ enthält [14].
} 

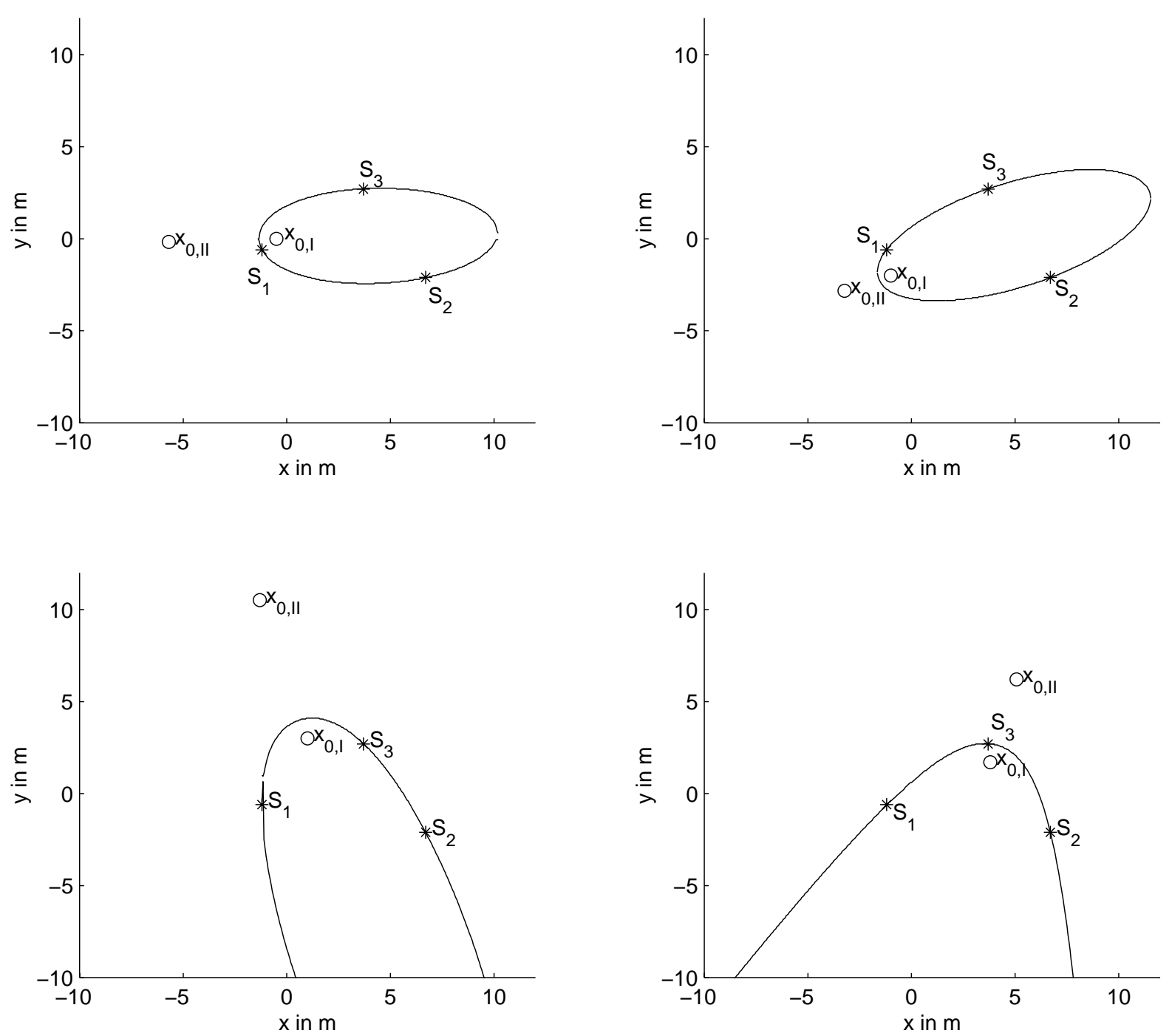

Abb. 4.9: Projizierte Konzentrationsschnittlinien für vier verschiedene Quellorte $\left(x_{0, I}\right)$ mit zugehörigen scheinbaren Quellorten $x_{0, I I}$ (links oben passend zu Abb. 4.8 bzw. Beispiel 4.2).

Im Fall reiner Diffusion genügt es, wenn sich der zusätzliche Sensor nicht auf dem Umkreis der anderen Sensoren (Konzentrationsschnittlinie) befand. Im Fall von DiffusionAdvektion hängen Lage und Form der Konzentrationsschnittlinie vom Quellort ab (Abb. 4.9). Wenn alle möglichen Quellorte berücksichtigt werden, füllt die Menge der zugehörigen Konzentrationsschnittlinien nahezu das gesamte Überwachungsgebiet aus. Allerdings bleibt das Innere des Dreiecks mit den Ecken $\left(\mathbf{x}_{1}, \mathbf{x}_{2}, \mathbf{x}_{3}\right)$ davon unberührt, da die Schnittlinien konvexe Mengen umranden. Das Dreieck ist aber gerade die konvexe Hülle der Sensorstandorte, oder mit anderen Worten, die kleinste Schnittmenge aller durch die Konzentrationsschnittlinien bestimmten konvexen Mengen [14]. Abb. 4.10 zeigt die Konzentrationsschnittlinien gemäß Abb. 4.9 sowie die konvexe Hülle (Dreieck) der Sensoren. 


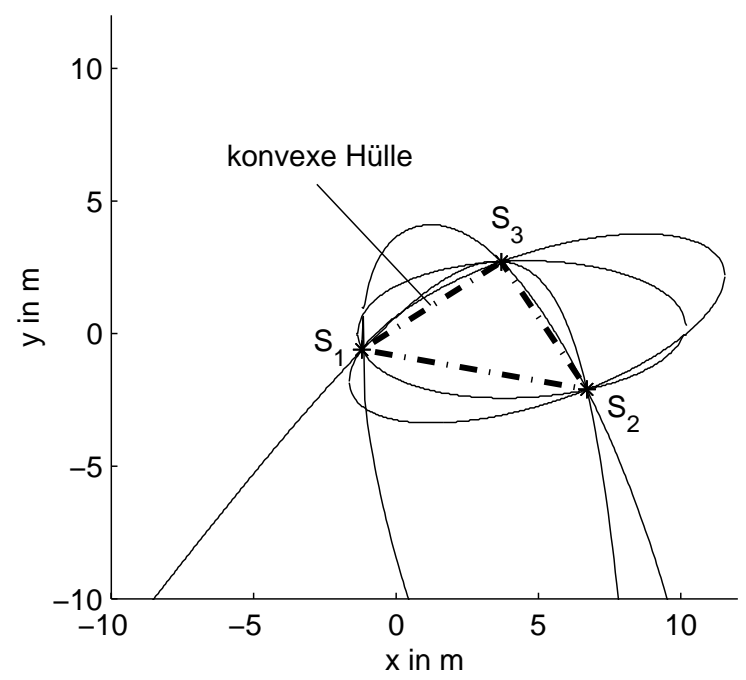

Abb. 4.10: Projizierte Konzentrationsschnittlinien für vier verschiedene Quellorte sowie konvexe Hülle der Sensorstandorte.

Daraus folgt, dass es keinen Quellort gibt, für den die zugehörige Konzentrationsschnittlinie die konvexe Hülle schneidet. Mit einem zusätzlichen Sensor innerhalb der konvexen Hülle der anderen Sensoren ist also stets eine Unterscheidung zwischen wahrem und scheinbarem Quellort möglich. Bei der Ebenenkonfiguration muss der vierte Sensor somit innerhalb des Dreiecks $\left(\mathbf{x}_{1}, \mathbf{x}_{2}, \mathbf{x}_{3}\right)$ liegen (Abb. 4.11 (links)). Bei der Raumkonfiguration bildet das Tetraeder mit den Ecken $\left(\mathbf{x}_{1}, \mathbf{x}_{2}, \mathbf{x}_{3}, \mathbf{x}_{4}\right)$ die konvexe Hülle (Abb. 4.11 (rechts)), in der der fünfte Sensor liegen muss.
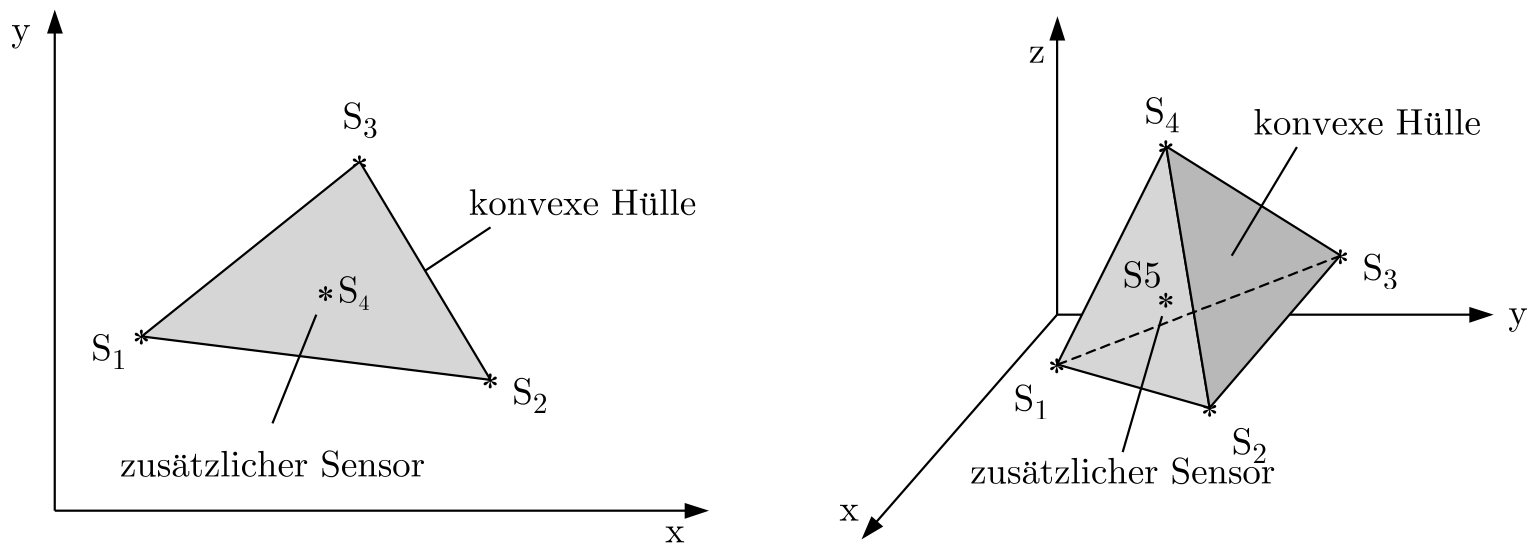

Abb. 4.11: Konvexe Hülle der Sensoren bei Ebenenkonfiguration (links) und bei Raumkonfiguration (rechts). Ein zusätzlicher Sensor zur Unterscheidung zwischen wahrem und scheinbarem Quellort muss innerhalb dieser konvexen Hülle liegen. 


\subsubsection{Zur Existenz von Lösungen im gestörten Fall}

Im ungestörten Fall existiert für den Quellort bei Verwenden minimaler Sensoranzahl stets mindestens eine Lösung, nämlich der wahre Quellort. Bei der Quellenlokalisierung im gestörten Fall auf der Basis der geschätzten Sensor-Quell-Abstände $\hat{d}_{i}$ kann es dazu kommen, dass (4.29) inkonsistent wird und keine Lösung für den Quellort existiert. Das verdeutlicht Abb. 4.12 für die Ebenenkonfiguration nach Beispiel 4.2. Die Schnittkurven für die Sensorpaare 1,2 und 1,3 schneiden sich nicht. Im Gegensatz zum Fall reiner Diffusion lassen sich hier keine analytischen Kriterien für die Existenz einer Lösung angeben.

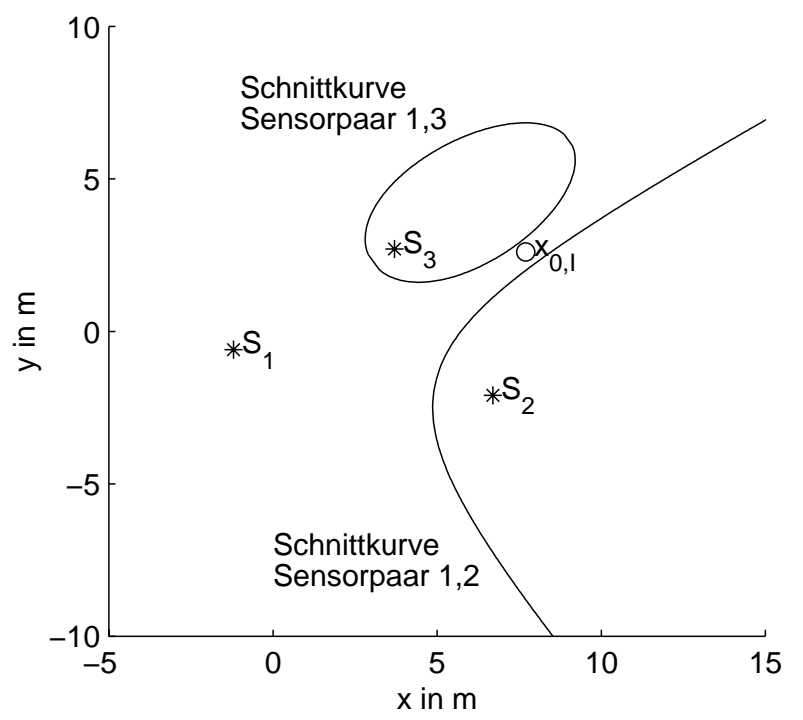

Abb. 4.12: Schnittkurven für Sensorpaare 1,2 und 1,3 schneiden sich im ungünstig gestörten Fall nicht.

\subsection{Quellortschätzung im gestörten Fall}

Im vorangegangenen Abschnitt wurde die Quellenlokalisierung bei Diffusion-Advektion für den ungestörten Fall auf der Basis minimaler Sensoranzahl behandelt. Im Abschnitt 4.3.3 wurde festgestellt, dass im gestörten Fall auch bei minimaler Sensoranzahl keine Lösung existieren muss. Selbst wenn eine Lösung existiert, hat die Verwendung der minimalen Sensoranzahl den Nachteil, dass jeder Konzentrationsmessfehler direkt einen Fehler bei der Quellenlokalisierung bewirkt. Aus diesem Grund ist es sinnvoll, im gestörten Fall eine größere Anzahl $p>m$ von Sensoren zu verwenden. Diese erlauben einen Fehlerausgleich und bei geeigneter Standortwahl eine Unterscheidung zwischen wahrem und scheinbarem Quellort. Das Gleichungssystem (4.29) wird im gestörten Fall zu

$$
\hat{d}_{i}\left(x_{0}, q_{0}\right)-\left\|\mathbf{x}_{i}-\mathbf{x}_{0}\right\|_{2}=0 ; \quad i=1, \ldots, p .
$$


Es ist mit $p>m$ überbestimmt und wegen der Störungen i. Allg. inkonsistent. Die Mengen $\mathcal{P}_{i}$ (Ovale), auf denen sich aus der Sicht der einzelnen Sensoren die Quelle befinden kann, besitzen folglich keinen gemeinsamen Schnittpunkt mehr (Abb. 4.13).

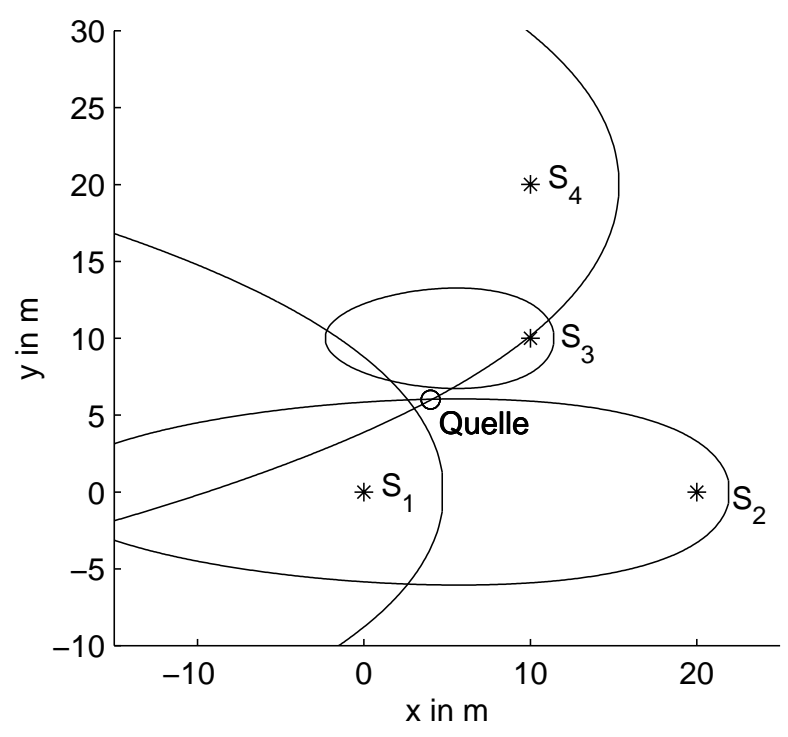

Abb. 4.13: Mengen $\mathcal{P}_{i}$ (Ovale) für alle $p$ Sensoren, die im gestörten Fall keinen gemeinsamen Schnittpunkt besitzen.

Um dennoch eine Lösung für den Quellort zu erhalten, lässt sich auf der Basis von (4.30) ein Optimierungsproblem

$$
\sum_{i=1}^{p}\left(\hat{d}_{i}\left(x_{0}, q_{0}\right)-\left\|\mathbf{x}_{i}-\mathbf{x}_{0}\right\|_{2}\right)^{2} \stackrel{!}{=} \operatorname{Min}_{\mathbf{x}_{0}, q_{0}}=: J
$$

stellen. Dessen Lösung erfolgt iterativ durch Gradientenverfahren, wobei für $p(p>m)$ Sensoren ein eindeutiges globales Minimum entsteht, wie das nachfolgende Beispiel verdeutlicht.

Beispiel 4.3 Für die in Abb. 4.2 bzw. 4.13 dargestellte Ebenenkonfiguration mit $\mathbf{x}_{1}=$ $(0 \mathrm{~m}, 0 \mathrm{~m})^{T}, \mathbf{x}_{2}=(20 \mathrm{~m}, 0 \mathrm{~m})^{T}, \mathbf{x}_{3}=(10 \mathrm{~m}, 10 \mathrm{~m})^{T}$ und $\mathbf{x}_{4}=(10 \mathrm{~m}, 20 \mathrm{~m})^{T}$ sowie einer Quelle bei $\mathbf{x}_{0}=(4 \mathrm{~m}, 6 \mathrm{~m})^{T}$ wird $J$ nach (4.31) berechnet. Das Gütegebirge $J\left(x_{0}, y_{0}\right)$ für festes $q_{0}$ zeigt Abb. 4.14 (links) für den ungestörten Fall. Im Vergleich dazu zeigt Abb. 4.14 (rechts) das Gütegebirge, das bei Verwenden des ausgangsfehlerbasierten Standardverfahrens für den ungestörten Fall entsteht.

Anhand der Gütegebirge in Beispiel 4.3 ist zu erkennen, dass die iterative Optimierung beim zweistufigen Verfahren unproblematisch ist. Es existiert ein eindeutiges globales Minimum im gesuchten Punkt $\mathbf{x}_{0}=(4 \mathrm{~m}, 6 \mathrm{~m})^{T}$. 

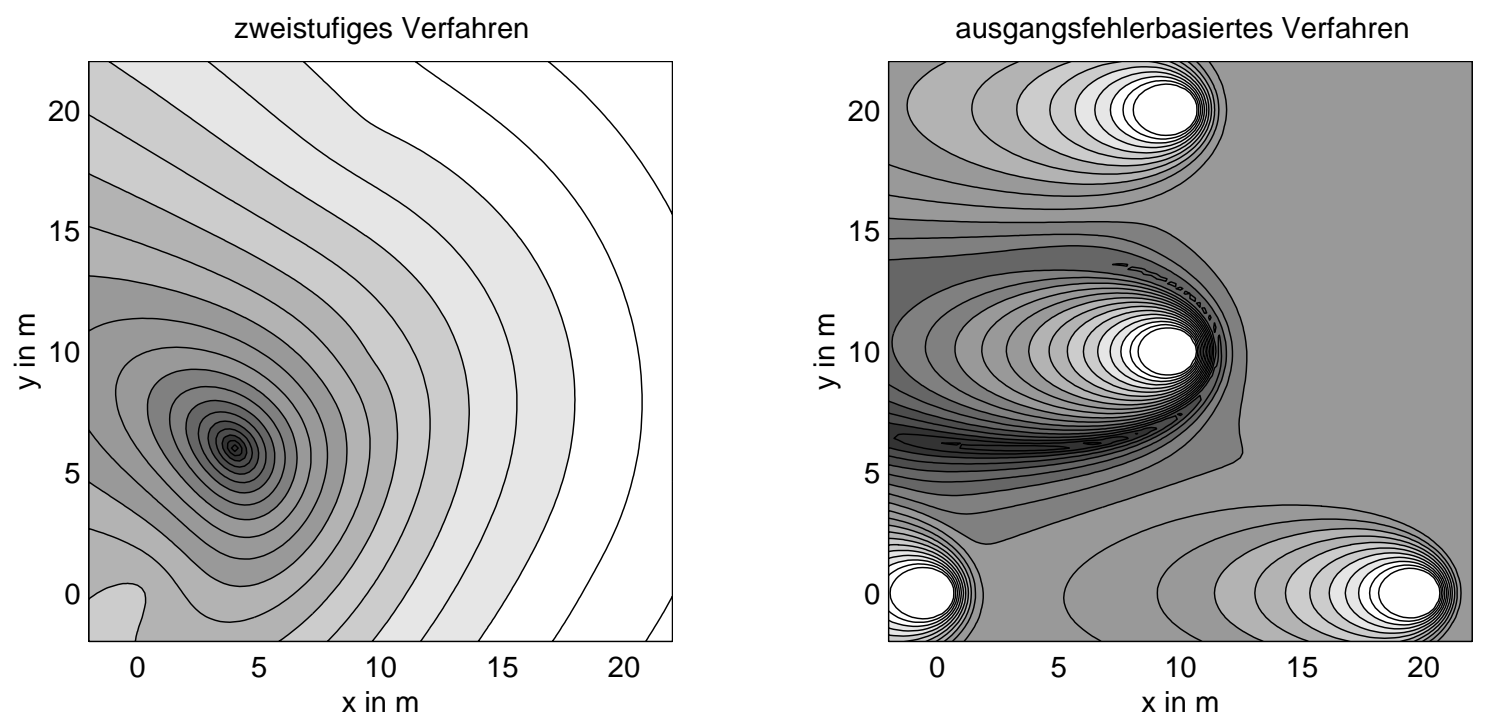

Abb. 4.14: Gütegebirge (logarithmisch) für das zweistufige Verfahren (4.31) (links) und für das ausgangsfehlerbasierte Standardverfahren (1.1) (rechts).

Mit dem ausgangsfehlerbasierten Standardverfahren ergibt sich hingegen ein Gütegebirge, das lokale uneigentliche ${ }^{4}$ Maxima in den Sensorstandorten und zudem Nebenminima besitzt, in denen iterative Optimierungsverfahren abbrechen und somit eine falsche Lösung liefern. Weiterhin existieren beim ausgangsfehlerbasierten Verfahren enge Täler, die die iterative Optimierung erschweren.

\subsection{Wichtung}

Durch (4.31) wird die Minimierung der Summe der Abstandsquadrate vom geschätzten Quellort zu allen Ovalen im geometrischen Sinne gefordert. Der Ausgleich erfolgt bei diesem Problem offensichtlich in der Weise

$$
\sum_{i=1}^{p}\left(\Delta \hat{d}_{i}\left(x_{0}, q_{0}\right)\right)^{2} \stackrel{!}{=} \underset{\mathbf{x}_{0}, q_{0}}{\operatorname{Min}} .
$$

Damit werden also Änderungen $\Delta \hat{d}_{i}\left(x_{0}, q_{0}\right)$ für jeden Sensor $i$ gleich bewertet.

Die Störungen treten jedoch in den Konzentrationsmessungen auf (z. B. Kalibrierfehler), weshalb es sinnvoller ist, einen Ausgleich bezüglich der Konzentrationen

$$
\sum_{i=1}^{p}\left(\Delta C_{i}\right)^{2} \stackrel{!}{=} \operatorname{Min}_{\mathbf{x}_{0}, q_{0}}
$$

\footnotetext{
${ }^{4}$ Das Adjektiv uneigentlich verweist darauf, dass $J$ den Wert $\infty$ annimmt. Da dieser nicht zum Wertebereich von $J$ gehört, handelt es sich um kein Maximum im eigentlichen Sinn.
} 
anzustreben, wie er durch das einstufige Ausgangsfehlerverfahren erzielt wird. Lassen sich Wichtungsfaktoren $w_{i}$ finden, die im linearisierten Fall dazu führen, dass

$$
\Delta C_{i} \approx w_{i} \cdot \Delta \hat{d}_{i}\left(x_{0}, q_{0}\right)
$$

gilt, dann kann (4.31) zu

$$
\sum_{i=1}^{p}\left(w_{i}\left(\hat{d}_{i}\left(x_{0}, q_{0}\right)-\left\|\mathbf{x}_{i}-\mathbf{x}_{0}\right\|_{2}\right)\right)^{2} \stackrel{!}{=} \underset{\mathbf{x}_{0}, q_{0}}{\operatorname{Min}}=: J
$$

erweitert werden. In Näherung verhält sich dann dieses Problem wie ein Ausgangsfehlerproblem in $C_{i}$. Um die Wichtungsfaktoren zu bestimmen, wird wie in Abschnitt $3.5 \mathrm{im}$ Sinne der Fehlerrechnung vorgegangen. Es folgt

$$
\Delta \hat{d}_{i}\left(x_{0}, q_{0}\right) \approx-\frac{1}{C_{i}} \cdot \frac{2 K}{v_{x}} \cdot \frac{\hat{d}_{i}\left(x_{0}, q_{0}\right) \cdot \frac{v_{x}}{2 K}}{\hat{d}_{i}\left(x_{0}, q_{0}\right) \cdot \frac{v_{x}}{2 K}+1} \cdot \Delta C_{i} .
$$

Für den Grenzfall $v_{x} \rightarrow 0$ gilt

$$
\Delta \hat{d}_{i}\left(x_{0}, q_{0}\right) \approx-\frac{q}{4 \pi K} \cdot \frac{1}{C^{2}} \cdot \Delta C_{i} \quad \text { also } \quad w_{i}=-C_{i}^{2} \cdot \frac{4 \pi K}{q_{0}}
$$

was erwartungsgemäß der Wichtung für den Diffusionsfall entspricht, vgl. (3.92).

Aus (4.36) folgt der optimale Wichtungsfaktor

$$
w_{i}=C_{i} \cdot \frac{\hat{d}_{i}\left(x_{0}, q_{0}\right) \cdot \frac{v_{x}}{2 K}+1}{\hat{d}_{i}\left(x_{0}, q_{0}\right) \cdot \frac{v_{x}}{2 K}},
$$

der jedoch abhängig von den Größen $x_{0}$ und $q_{0}$ ist, die zu Beginn der Optimierung nicht bekannt sind. Ist das Verhältnis $v / K$ hinreichend groß und befindet sich die Quelle nicht in unmittelbarer Nähe des Sensors $i$, so gilt $\hat{d}_{i}\left(x_{0}, q_{0}\right) \cdot \frac{v_{x}}{2 K} \gg 1$ und damit in Näherung

$$
w_{i}=C_{i}
$$

Diese vereinfachten Wichtungsfaktoren lassen sich für die Optimierung nutzen. Liegen nach der Optimierung Schätzwerte für $\hat{d}_{i}\left(x_{0}, q_{0}\right)$ vor, können die Wichtungsfaktoren nach (4.38) ermittelt und für eine weitere Feinoptimierung (4.35) genutzt werden.

Beispiel 4.4 Für die in Abb. 4.15 dargestellte Ebenenkonfiguration wird eine Lokalisierung auf der Basis der stationären Konzentrationen durchgeführt. Dabei werden die Konzentrationen mit deterministischen Absolutfehlern beaufschlagt, wie sie durch Kalibrierfehler entstehen. Es wird also zum Konzentrationswert jedes Sensors eine feste positive bzw. negative Konzentration (Kalibrierfehler) addiert (vgl. Beispiel 3.3). Die Ergebnisse, die 
dabei sowohl mit dem ungewichteten Verfahren als auch mit dem gewichteten Verfahren erzielt werden, zeigt Abb. 4.15. Zudem sind die Ergebnisse des Ausgangsfehlerverfahrens dargestellt, das hier stets das globale Minimum findet und nicht in Nebenminima abbricht, da hier die wahren Werte für Quellort und-intensität als Startwerte vorgegeben wurden. Werden Startwerte in der Nähe der wahren Lösung gewählt, so findet das Ausgangsfehlerverfahren ebenfalls das globale Minimum. Wird jedoch als Startwert beispielsweise $(6,13)$ gewählt, bricht das Verfahren im lokalen Minimum bei $(8.4,13.2)$ ab (vgl. Abb. 4.14).

Beispiel 4.4 zeigt, dass mit dem gewichteten Verfahren deutlich bessere Ergebnisse erzielt werden als mit dem ungewichteten Verfahren (maximale Abweichung zwischen wahrem und geschätztem Quellort $0.4 \mathrm{~m}$ beim gewichteten Verfahren gegenüber $2.9 \mathrm{~m}$ beim ungewichteten Verfahren). Im Vergleich zum Ausgangsfehlerverfahren sind keine wesentlichen Unterschiede zu erkennen.
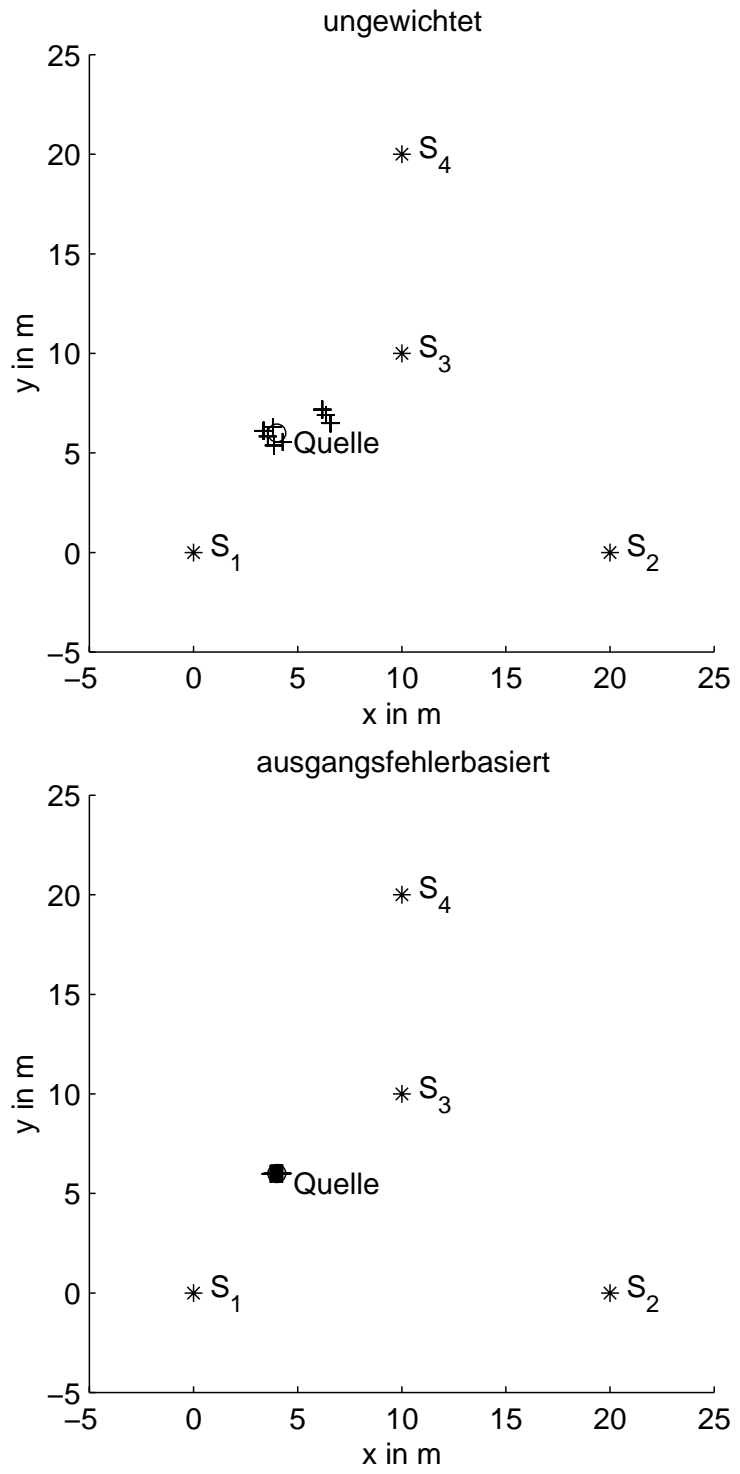

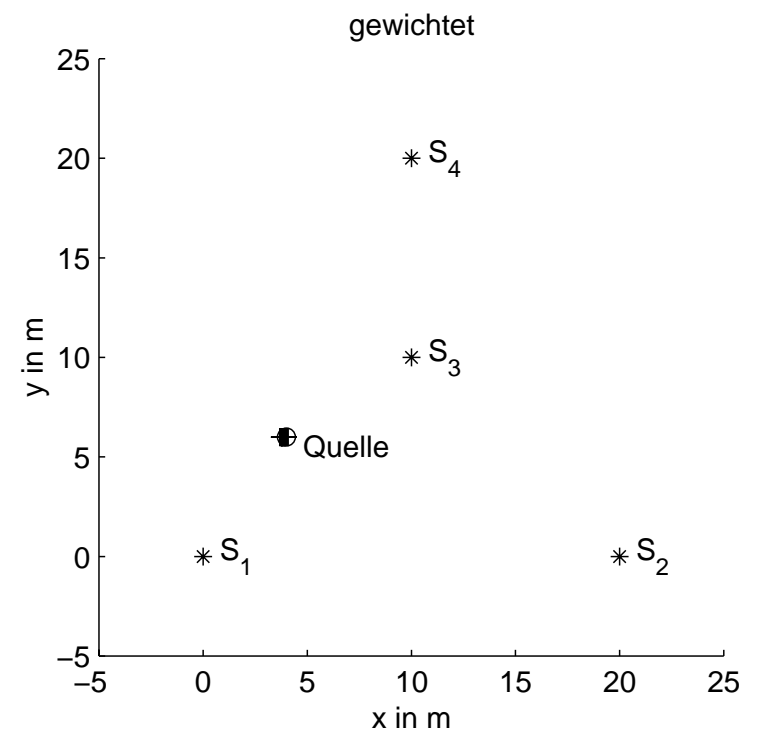

Abb. 4.15: Abweichungen der Quellortschätzung beim ungewichteten Verfahren, beim gewichteten Verfahren und beim ausgangsfehlerbasierten Standardverfahren. (Als Startwerte für das ausgangsfehlerbasierte Standardverfahren wurden die wahren Parameter verwendet; damit wird das globale Minimum gefunden.) 


\subsection{Fehler der Quellortschätzung mit stationären Konzentrationen}

In diesem Abschnitt wird anhand eines Simulationsbeispiels untersucht, welche Abweichungen durch eine Quellenlokalisierung auf der Basis stationärer Endwerte entstehen, wenn nach endlicher Messzeit die zuletzt gemessenen Konzentrationen als Schätzwerte für die stationären Konzentrationswerte verwendet werden. Für die Untersuchungen wird die im Abb. 2.5 (links) dargestellte Anordnung von Sensoren und Quelle mit den sich daraus ergebenden Konzentrationsverläufen gemäß Abb. 2.5 (rechts) verwendet.

Werden nun die zu jedem Zeitpunkt gemessenen Konzentrationswerte als Schätzwerte für die stationären Konzentrationen angesehen, entsteht ein systematischer Fehler, da die Konzentrationen tatsächlich noch steigen. Damit ergibt sich auch im ungestörten Fall eine Abweichung zwischen tatsächlichem und geschätztem Quellort. Diese zeitabhängige Abweichung ist in Abb. 4.16 für verschiedene Strömungsgeschwindigkeiten $v_{x}$ und konstanten Diffusionskoeffizienten $K=0.2 \mathrm{~m}^{2} / \mathrm{s}$ dargestellt.

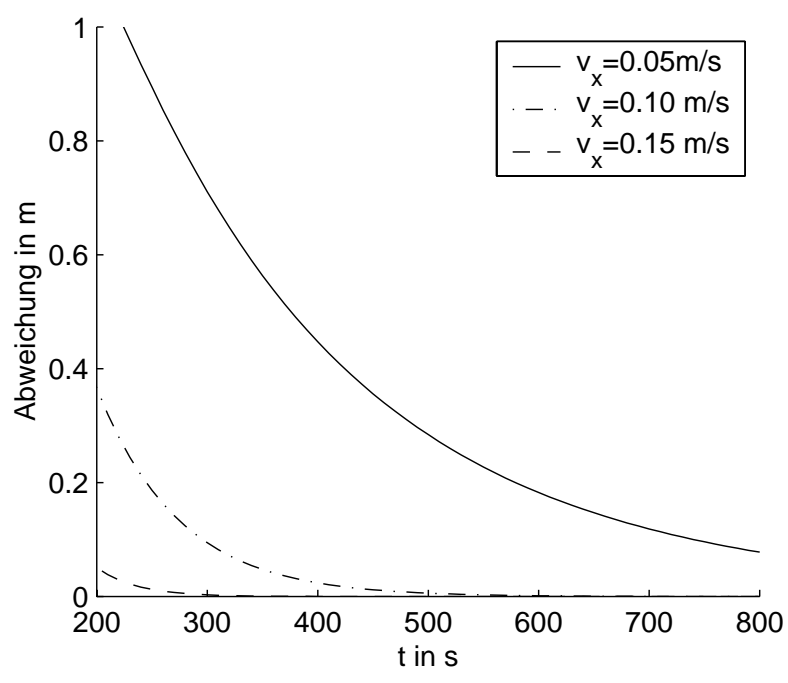

Abb. 4.16: Abweichung des geschätzten Quellorts vom wahren Wert in Abhängigkeit von der Beobachtungszeit bei Schätzung auf der Basis stationärer Konzentrationen im ungestörten Fall für verschiedene Strömungsgeschwindigkeiten $v_{x}\left(K=0.2 \mathrm{~m}^{2} / \mathrm{s}\right)$.

Die Abweichung zwischen wahrem und geschätztem Quellort sinkt erwartungsgemäß mit der Zeit, da sich die Konzentrationen den stationären Werten nähern. Eine Schätzung des Quellorts kann also nur nach einer bestimmten Zeit nach Beginn der Emission auf der Basis stationärer Konzentrationswerte erfolgen. Die notwendige Zeit verkürzt sich aber mit steigender Strömungsgeschwindigkeit $v_{x}$. 


\subsection{Zusammenfassung}

In diesem Kapitel wurde ein neues Verfahren zur Quellenlokalisierung für den Fall von Diffusion-Advektion entwickelt. Es basiert auf einem geometrischen Zugang, bei dem zunächst für jeden Sensor $i$ einzeln die Menge $\mathcal{P}_{i}$ bestimmt wird, auf der sich aus der Sicht dieses Sensors die Quelle befinden kann. Durch Betrachten mehrerer $\mathcal{P}_{i}$ lässt sich daraus der Quellort schätzen.

Im Fall von Diffusion-Advektion bildet diese Menge für jeden Sensor ein Oval um den Sensorstandort. Form und Größe dieses Ovals hängen von der gemessenen Konzentration $C_{i}$ am jeweiligen Sensor, der Quellintensität $q_{0}$, dem Diffusionskoeffizient $K$ und der Strömungsgeschwindigkeit $v_{x}$ ab. Es wird gezeigt, dass eine Beschreibung des Ovals durch den verallgemeinerten Sensor-Quell-Abstand $d_{i}$ möglich ist. Falls $v_{x}$ und $K$ durch Vorabexperimente identifiziert oder online gemessen werden, ist $d_{i}$ nur noch von der gemessenen Konzentration und von der Quellintensität $q_{0}$ abhängig.

Im Diffusionsfall bewirkte $q_{0}$ eine lineare Skalierung von $d_{i}$, weshalb eine Zerlegung in einen skalierbaren Sensor-Quell-Abstand und einen linearen Skalierungsfaktor erfolgen konnte. Im Advektionsfall ist $d_{i}$ eine komplizierte nichtlineare Funktion von $q_{0}$ und der $x$-Komponente des Quellorts $x_{0}$.

Um den Quellort zu ermitteln, wird ein nichtlineares Gleichungssystem mit allen $d_{i}\left(x_{0}, q_{0}\right)$ formuliert, das sich durch iterative Verfahren lösen lässt. Die iterativ gefundene Lösung enthält zugleich einen Schätzwert für die Quellintensität.

Für den ungestörten Fall mit minimaler Sensoranzahl wird mit Hilfe von Schnittkurven gezeigt, dass es - wie auch im Diffusionsfall - entweder eine Lösung oder zwei Lösungen (eine wahre und eine scheinbare) Lösung für das Quellenlokalisierungsproblem gibt. Allerdings kann bei zwei möglichen Lösungen die Lage der zweiten Lösung nur noch für den Fall einer Linienkonfiguration analytisch angegeben werden. Es wird jedoch gezeigt, dass durch Platzieren eines weiteren Sensors innerhalb der konvexen Hülle der anderen Sensoren auch bei der Ebenen-, Raum- und Halbraumkonfiguration stets eine Unterscheidung zwischen wahrem und scheinbarem Quellort möglich ist. Unterscheidungsprobleme zwischen wahrem und scheinbarem Quellort bei gestörten Konzentrationsmessungen und ungünstigen Sensorstandorten können im Fall von Diffusion-Advektion analog zum Diffusionsfall auftreten, wobei hier kein explizites Beispiel angegeben wird. 
Im gestörten Fall mit beliebiger Sensoranzahl entsteht ein überbestimmtes, i. Allg. inkonsistentes, nichtlineares Gleichungssystem, das in ein Optimierungsproblem für den Quellort und die Quellintensität umgeformt wird. Dieses Optimierungsproblem lässt sich ebenfalls iterativ lösen.

Durch Einführen von Wichtungsfaktoren wird berücksichtigt, dass ein Ausgleich in den gestörten Konzentrationen und nicht in den Sensor-Quell-Abständen stattfindet. Da mit dieser Wichtung aus statistischer Sicht annähernd eine Maximum-likelihood-Schätzung erfolgt, sind damit in Näherung gleich gute statistische Eigenschaften wie beim klassischen ausgangsfehlerbasierten Verfahren (Maximum-likelihood-Schätzung) zu erwarten, was durch die gezeigten Simulationsbeispiele deutlich wird. Dabei besitzt das neue Verfahren nicht die numerischen Probleme des Ausgangsfehlerverfahrens. 


\section{Simulative und praktische Erprobung der neuen Verfahren}

\subsection{Komplexbeispiel Diffusion}

In diesem Abschnitt soll die Leistungsfähigkeit des neuen Verfahrens an einem realitätsnahen Simulationsbeispiel demonstriert werden. Dazu wird die in Abb. 3.15 verwendete Ebenenkonfiguration genutzt. Es wird angenommen, dass alle Sensoren einen Kalibrierfehler besitzen, der zu einer dauerhaften systematischen Abweichung der Messung vom wahren Wert führt. Der Kalibrierfehler beträgt für die Sensoren 1 und $2 \Delta C=1 \mathrm{mg} / \mathrm{m}^{3}$ und für die Sensoren 3 und $4 \Delta C=-1 \mathrm{mg} / \mathrm{m}^{3}$. Dies entspricht einer relativen Abweichung von $5 \%$ bezogen auf den Messbereich $\left(0-20 \mathrm{mg} / \mathrm{m}^{3}\right)$ der Sensoren und $15 \%$ bezogen auf den mittleren stationären Konzentrationswert aller Sensoren. Zudem tritt unkorreliertes stationäres normalverteiltes Rauschen als zusätzliche Störung mit einer Stärke ${ }^{1}$ von $\pm 1.2 \mathrm{mg} / \mathrm{m}^{3}, \pm 2.4 \mathrm{mg} / \mathrm{m}^{3}$ bzw. $\pm 4.8 \mathrm{mg} / \mathrm{m}^{3}$ auf. Die Simulationen werden für die Quellorte $\mathbf{x}_{0}=(5 \mathrm{~m}, 10 \mathrm{~m})$ und $\mathbf{x}_{0}=(3 \mathrm{~m}, 5 \mathrm{~m})$ durchgeführt.

$600 \mathrm{~s}$ nach Beginn der Emission (Startzeit $t_{0}=0 \mathrm{~s}$, Quellintensität $q_{0}=300 \mathrm{mg} / \mathrm{s}$, Diffusionskoeffizient $K=0.2 \mathrm{~m}^{2} / \mathrm{s}$ ) wird der Quellort auf der Basis stationärer als auch instationärer Konzentrationen mit den gewichteten Verfahren geschätzt.

Für jedes Beispiel erfolgen 200 Simulationen, wobei aus Darstellungsgründen in Abb. 5.1 und Abb. 5.2 jeweils nur die Ergebnisse von 20 zufällig ausgewählten Simulationen gezeigt werden. Tabelle 5.1 gibt einen Überblick über die Ergebnisse, die auf der Basis der jeweils 200 Simulationen ermittelt wurden.

\footnotetext{
${ }^{1}$ Die Stärke einer unkorrelierten, stationären, normalverteilten Störung wird hier durch das Intervall um den ungestörten Wert definiert, in dem sich 99.7\% aller gestörten Werte befinden ( $3 \sigma$-Regel).
} 

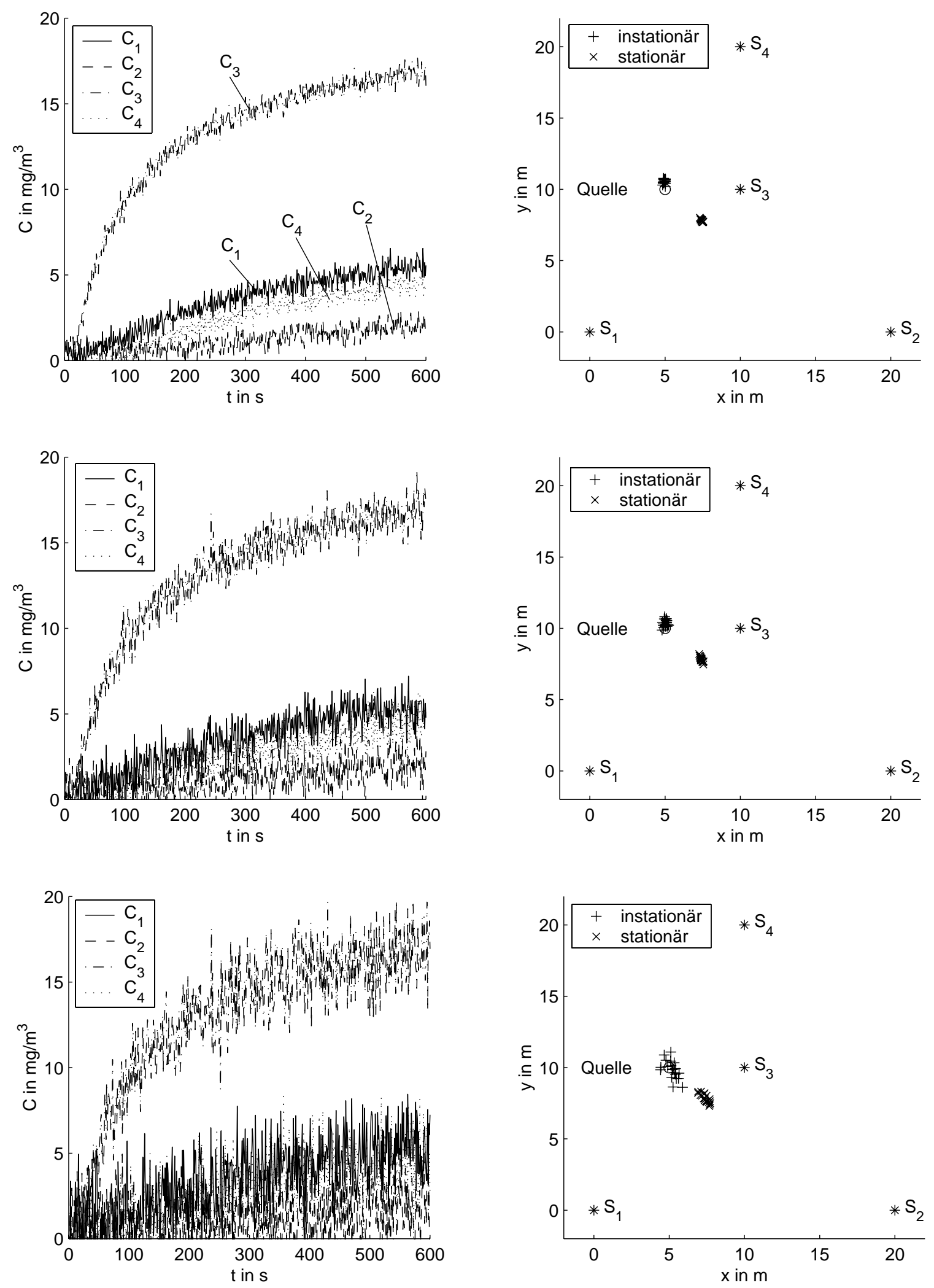

Abb. 5.1: Konzentrationsverläufe (links) sowie Quellortschätzungen für 20 Simulationen (rechts) für Quellort $\mathbf{x}_{0}=(5 \mathrm{~m}, 10 \mathrm{~m})^{T}$ und Störung mit $\pm 1.2 \mathrm{mg} / \mathrm{m}^{3}, \pm 2.4 \mathrm{mg} / \mathrm{m}^{3}$ sowie $\pm 4.8 \mathrm{mg} / \mathrm{m}^{3}$. 

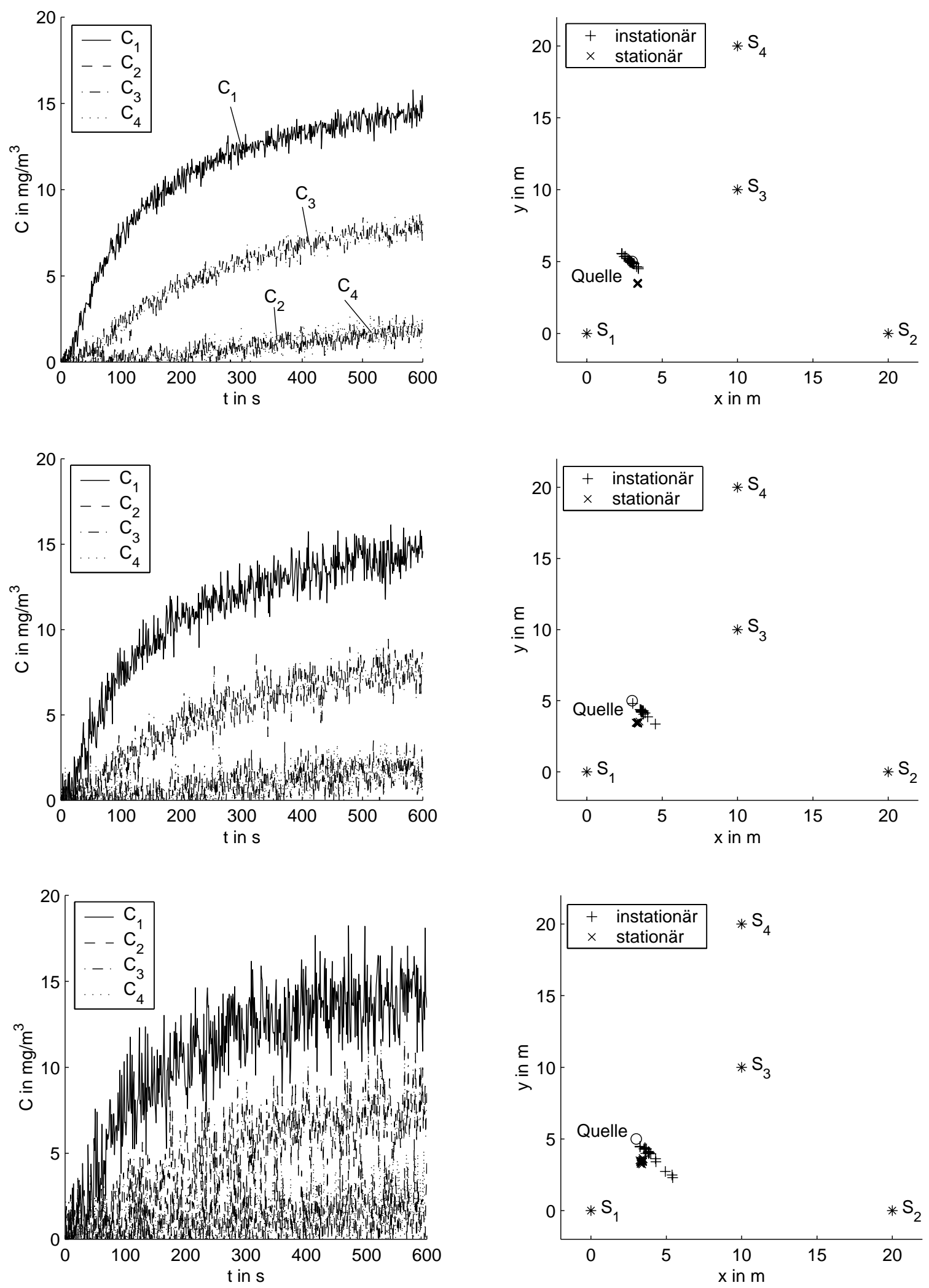

Abb. 5.2: Konzentrationsverläufe (links) sowie Quellortschätzungen für 20 Simulationen (rechts) für Quellort $\mathbf{x}_{0}=(3 \mathrm{~m}, 5 \mathrm{~m})^{T}$ und Störung mit $\pm 1.2 \mathrm{mg} / \mathrm{m}^{3}, \pm 2.4 \mathrm{mg} / \mathrm{m}^{3}$ sowie $\pm 4.8 \mathrm{mg} / \mathrm{m}^{3}$. 


\begin{tabular}{|c|c|c|c|c|c|}
\hline $\begin{array}{c}\text { Quellort } \\
\mathbf{x}_{0}^{T}\end{array}$ & Störung & Verfahren & $\begin{array}{c}\text { Mittelwert der } \\
\text { Abweichung } \\
\left\|\hat{\mathbf{x}}_{0}-\mathbf{x}_{0}\right\|_{2}\end{array}$ & $\begin{array}{c}\text { Streuung der } \\
\text { Abweichung } \\
\left\|\hat{\mathbf{x}}_{0}-\mathbf{x}_{0}\right\|_{2}\end{array}$ & $\begin{array}{c}\text { mittlere } \\
\text { Rechenzeit }\end{array}$ \\
\hline \multirow{8}{*}{$(5 \mathrm{~m}, 10 \mathrm{~m})$} & \multirow[t]{2}{*}{ $\pm 0 \mathrm{mg} / \mathrm{m}^{3}$} & stationär & $3.20 \mathrm{~m}$ & - & $0.02 \mathrm{~s}$ \\
\hline & & instationär & $0.58 \mathrm{~m}$ & - & $11 \mathrm{~s}$ \\
\hline & \multirow[t]{2}{*}{ $\pm 1.2 \mathrm{mg} / \mathrm{m}^{3}$} & stationär & $3.28 \mathrm{~m}$ & $0.01 \mathrm{~m}^{2}$ & $0.02 \mathrm{~s}$ \\
\hline & & instationär & $0.59 \mathrm{~m}$ & $0.02 \mathrm{~m}^{2}$ & $12 \mathrm{~s}$ \\
\hline & \multirow[t]{2}{*}{ $\pm 2.4 \mathrm{mg} / \mathrm{m}^{3}$} & stationär & $3.52 \mathrm{~m}$ & $0.02 \mathrm{~m}^{2}$ & $0.02 \mathrm{~s}$ \\
\hline & & instationär & $0.68 \mathrm{~m}$ & $0.09 \mathrm{~m}^{2}$ & $14 \mathrm{~s}$ \\
\hline & \multirow[t]{2}{*}{ $\pm 4.8 \mathrm{mg} / \mathrm{m}^{3}$} & stationär & $3.81 \mathrm{~m}$ & $0.07 \mathrm{~m}^{2}$ & $0.02 \mathrm{~s}$ \\
\hline & & instationär & $0.84 \mathrm{~m}$ & $0.95 \mathrm{~m}^{2}$ & $19 \mathrm{~s}$ \\
\hline \multirow{8}{*}{$(3 \mathrm{~m}, 5 \mathrm{~m})$} & \multirow[t]{2}{*}{ $\pm 0 \mathrm{mg} / \mathrm{m}^{3}$} & stationär & $1.56 \mathrm{~m}$ & - & $0.02 \mathrm{~s}$ \\
\hline & & instationär & $0.38 \mathrm{~m}$ & - & $8 \mathrm{~s}$ \\
\hline & \multirow[t]{2}{*}{ $\pm 1.2 \mathrm{mg} / \mathrm{m}^{3}$} & stationär & $1.57 \mathrm{~m}$ & $0.01 \mathrm{~m}^{2}$ & $0.02 \mathrm{~s}$ \\
\hline & & instationär & $0.42 \mathrm{~m}$ & $0.17 \mathrm{~m}^{2}$ & $8 \mathrm{~s}$ \\
\hline & \multirow[t]{2}{*}{ $\pm 2.4 \mathrm{mg} / \mathrm{m}^{3}$} & stationär & $1.59 \mathrm{~m}$ & $0.01 \mathrm{~m}^{2}$ & $0.02 \mathrm{~s}$ \\
\hline & & instationär & $1.31 \mathrm{~m}$ & $0.87 \mathrm{~m}^{2}$ & $9 \mathrm{~s}$ \\
\hline & \multirow[t]{2}{*}{ $\pm 4.8 \mathrm{mg} / \mathrm{m}^{3}$} & stationär & $1.61 \mathrm{~m}$ & $0.02 \mathrm{~m}^{2}$ & $0.02 \mathrm{~s}$ \\
\hline & & instationär & $1.63 \mathrm{~m}$ & $1.18 \mathrm{~m}^{2}$ & $11 \mathrm{~s}$ \\
\hline
\end{tabular}

Tabelle 5.1: Mittlere Ergebnisse für jeweils 200 Simulationen pro Beispiel für die Verfahren mit stationären und instationären Konzentrationen.

Die Ergebnisse zeigen zunächst, dass die statistischen Eigenschaften der Quellortschätzung von der Lage des Quellorts abhängen.

Weiterhin ist zu erkennen, dass die deterministischen Kalibrierfehler wesentlichen Einfluss auf die mittlere Abweichung zwischen tatsächlichem und geschätztem Quellort haben (Simulation mit Rauschen Stärke 0mg $/ \mathrm{m}^{3}$ ). Das liegt daran, dass die Kalibrierfehler systematische Fehler sind, die durch einen Fehlerausgleich nicht unterdrückt werden können. Anders verhält es sich mit dem Rauschen. Da durch das gewichtete Quellenlokalisierungsverfahren, wie auch bei der Maximum-likelihood-Schätzung, ein Ausgleich in den Konzentrationen angestrebt wird, kann das unkorrelierte, stationäre, normalverteilte Rauschen zufriedenstellend kompensiert werden.

Dies gilt auch für die Schätzung auf der Basis stationärer Konzentrationen, da durch die Mittelwertbildung zur Berechnung dieser Konzentrationen ein gute Kompensation des Rauschens erfolgt. Jedoch besitzen die geschätzten Quellorte eine größere mittlere Abweichung als beim instationären Verfahren. Dies lässt sich damit begründen, dass nach 
600s die stationären Endwerte noch nicht erreicht sind (vgl. auch Abschnitt 3.7), was insbesondere für den Quellort $(5 \mathrm{~m}, 10 \mathrm{~m})$ deutlich wird.

Die Rechenzeit ${ }^{2}$ für die Verfahren mit instationären Konzentrationen ist wesentlich größer als bei den Verfahren mit stationären Konzentrationen, da beim instationären Verfahren eine Optimierung mit Hilfe aller Konzentrationsmesswerte für jeden Sensor durchgeführt wird. Beim stationären Verfahren werden nur die letzten Konzentrationsmesswerte für eine Mittelwertbildung verwendet.Die gezeigten Rechenzeiten bereiten praktisch keine Probleme, da sie im Vergleich zur Ausbreitungszeit klein sind.

\subsection{Komplexbeispiel Diffusion-Advektion}

Dieser Abschnitt untersucht ein Komplexbeispiel für die Quellenlokalisierung bei DiffusionAdvektion. Die Sensor-Quell-Anordnung und die Quellorte sind dabei identisch zum Komplexbeispiel für den Diffusionsfall.

Es wird wiederum angenommen, dass alle Sensoren einen Kalibrierfehler besitzen, der zu einer dauerhaften systematischen Abweichung der Messung vom wahren Wert führt. Der Kalibrierfehler beträgt für die Sensoren 1 und $2 \Delta C=1 \mathrm{mg} / \mathrm{m}^{3}$ und für die Sensoren 3 und $4 \Delta C=-1 \mathrm{mg} / \mathrm{m}^{3}$. Dies entspricht einer relativen Abweichung von $5 \%$ bezogen auf den Messbereich $\left(0-20 \mathrm{mg} / \mathrm{m}^{3}\right)$ der Sensoren und $20 \%^{3}$ bezogen auf den mittleren stationären Konzentrationswert aller Sensoren. Zusätzlich wird eine unkorrelierte stationäre normalverteilte Störung der Strömungsrichtung um $\pm 10^{\circ}$ und der Strömungsgeschwindigkeit um $\pm 0.02 \mathrm{~m} / \mathrm{s}$ (entspricht $\pm 20 \%$ ) angenommen. Weiterhin tritt unkorreliertes, stationäres, normalverteiltes Rauschen als zusätzliche Störung mit einer Stärke von $\pm 1.2 \mathrm{mg} / \mathrm{m}^{3}$, $\pm 2.4 \mathrm{mg} / \mathrm{m}^{3}$ bzw. $\pm 4.8 \mathrm{mg} / \mathrm{m}^{3}$ auf.

Nach einer Zeit von 600s nach Beginn der Emission (Startzeit $t_{0}=0 \mathrm{~s}$, Quellintensität $q_{0}=300 \mathrm{mg} / \mathrm{s}$, Diffusionskoeffizient $K=0.2 \mathrm{~m}^{2} / \mathrm{s}$, Strömungsgeschwindigkeit $v_{x}=$ $0.1 \mathrm{~m} / \mathrm{s}$ ) wird der Quellort auf der Basis stationärer Konzentrationen mit dem gewichteten Verfahren geschätzt. Für jedes Beispiel werden 200 Simulationen durchgeführt, wobei erneut aus Darstellungsgründen in Abb. 5.3 und Abb. 5.4 nur 20 zufällig ausgewählte Quellortschätzungen gezeigt werden.

\footnotetext{
${ }^{2}$ Alle Rechenzeitangaben beziehen sich auf Berechnungen mit Matlab auf einem Standard-PC mit $2 \mathrm{GHz}$ Taktfrequenz. Durch Implementieren der Algorithmen in ein Sensor-Managementsystem wie beispielsweise INSPECT-S [58, 74] ist eine Verringerung der Rechenzeiten zu erwarten.

${ }^{3}$ Die gleiche absolute Störung führte im Fall von Diffusion auf einen relativen Fehler von $15 \%$ bezogen auf den mittleren stationären Konzentrationswert aller Sensoren, da diese Konzentrationen dort größer waren.
} 
Tabelle 5.2 fasst die Ergebnisse aller Simulationen zusammen. Für die Untersuchungen wurde zunächst jede Störung und ihre Auswirkung auf die Quellortschätzung einzeln betrachtet. Auf der Basis der jeweils durchgeführten 200 Simulationen wurden Mittelwerte und Streuungen des Abstandes des geschätzten Quellorts zum tatsächlichen Quellort berechnet.

Es ist zu erkennen, dass die deterministischen Kalibrierfehler den größten Einfluss auf die mittlere Abweichung zwischen geschätztem und tatsächlichem Quellort haben, da sie nicht durch einen Fehlerausgleich unterdrückt werden können. Ebenfalls deutlichen Einfluss auf die mittlere Abweichung haben die Fehler der Strömungsrichtung und -geschwindigkeit, wobei die Strömungsrichtung einen größeren Einfluss als die -geschwindigkeit hat. Die Ergebnisse zeigen jedoch, dass eine grobe Kenntnis der Strömungsbedingungen genügt, um befriedigende Quellortschätzungen zu erhalten.

Bei den Simulationen mit allen Störungen und zusätzlichem Messrauschen wird deutlich, dass sich die mittleren Abweichungen der einzelnen Störungen nicht addieren müssen. Das lässt sich damit erklären, dass die mittleren Verschiebungen des geschätzten Quellorts zum tatsächlichen Quellort je nach Störungsart in verschiedene Richtungen erfolgen.

Die Quellortschätzungen streuen erwartungsgemäß um so stärker, je größer die Stärke des Rauschens $\left( \pm 1.2 \mathrm{mg} / \mathrm{m}^{3}, \pm 2.4 \mathrm{mg} / \mathrm{m}^{3}\right.$ bzw. $\left.\pm 4.8 \mathrm{mg} / \mathrm{m}^{3}\right)$ ist. Es wird jedoch sichtbar, dass auch bei starkem Rauschen mit einer Stärke von $\pm 4.8 \mathrm{mg} / \mathrm{m}^{3}$ zufriedenstellende Resultate erzielt werden. Dies lässt sich wiederum durch den guten Ausgleich von Konzentrationsmessfehlern, der durch das gewichtete Verfahren erzielt wird (annähernde Maximumlikelihood-Schätzung), erklären.

Weiterhin zeigt Tabelle 5.2, dass die Abweichungen auch abhängig vom Quellort sind, wobei für die untersuchten Quellorte die Unterschiede relativ klein sind. Die Abhängigkeit der statistischen Eigenschaften vom Quellort resultiert prinzipiell daraus, dass je nach Quellort verschieden hohe Konzentrationen an den Sensoren gemessen werden. Damit wirken sich die Störungen (z. B. Kalibrierfehler) unterschiedlich stark aus. Besonders deutlich wird das, wenn eine Quelle auf der strömungsabgewandten Seite der Sensoren betrachtet wird. Die entstehenden sehr kleinen Konzentrationen an den Sensoren können durch die Kalibrierfehler zu relativen Störungen im Bereich mehrerer 100\% führen. Eine Quellenlokalisierung mit Störungen ist bei Advektion also nur für Quellorte auf der strömungszugewandten Seite sinnvoll.

Die Rechenzeit für die Quellenlokalisierung betrug durchschnittlich 12 s. 

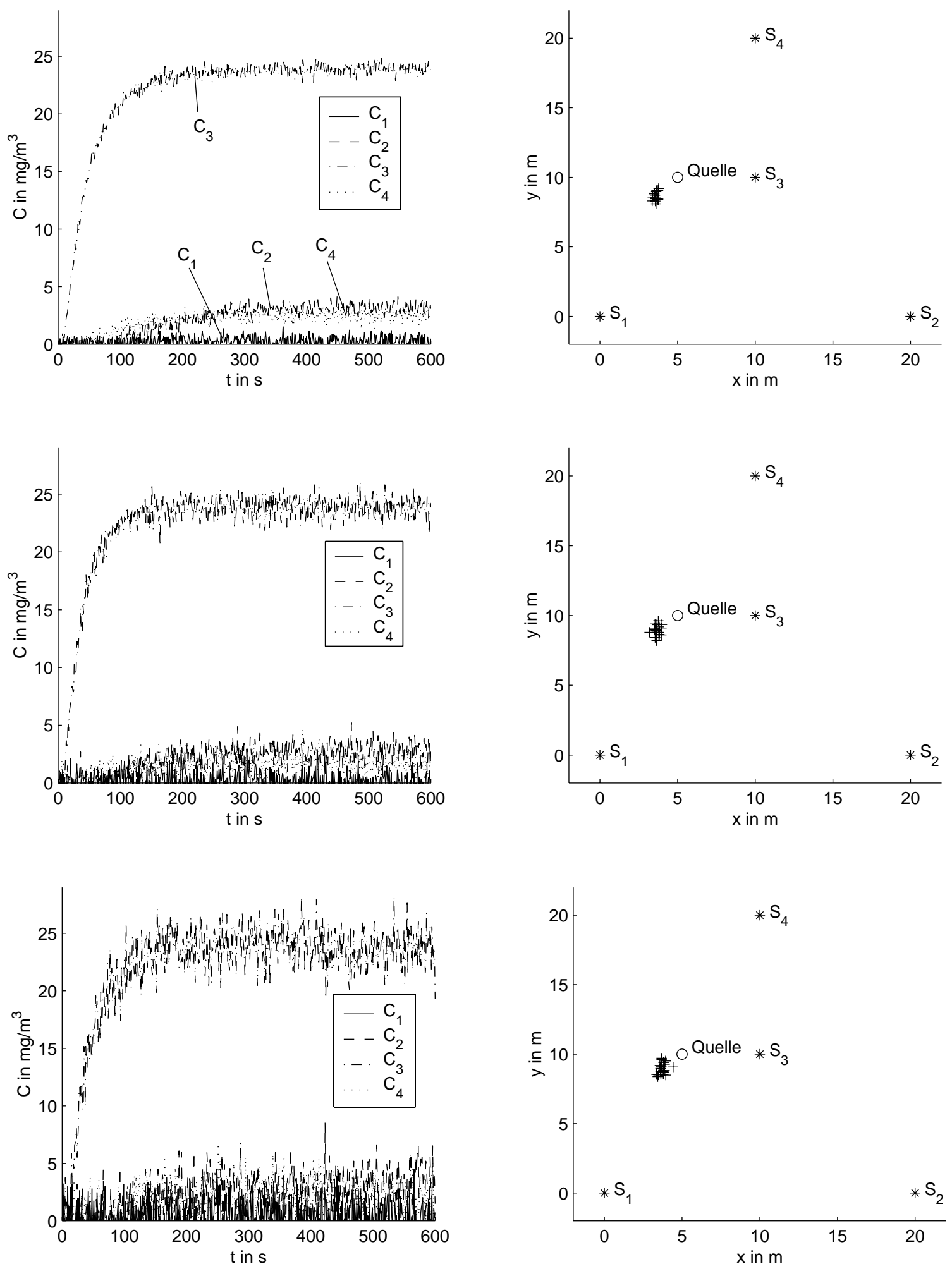

Abb. 5.3: Konzentrationsverläufe (links) sowie Quellortschätzungen für 20 Simulationen (rechts) für Quellort $\mathbf{x}_{0}=(3 \mathrm{~m}, 5 \mathrm{~m})^{T}$ und Störung mit $\pm 1.2 \mathrm{mg} / \mathrm{m}^{3}, \pm 2.4 \mathrm{mg} / \mathrm{m}^{3}$ sowie $\pm 4.8 \mathrm{mg} / \mathrm{m}^{3}$. 

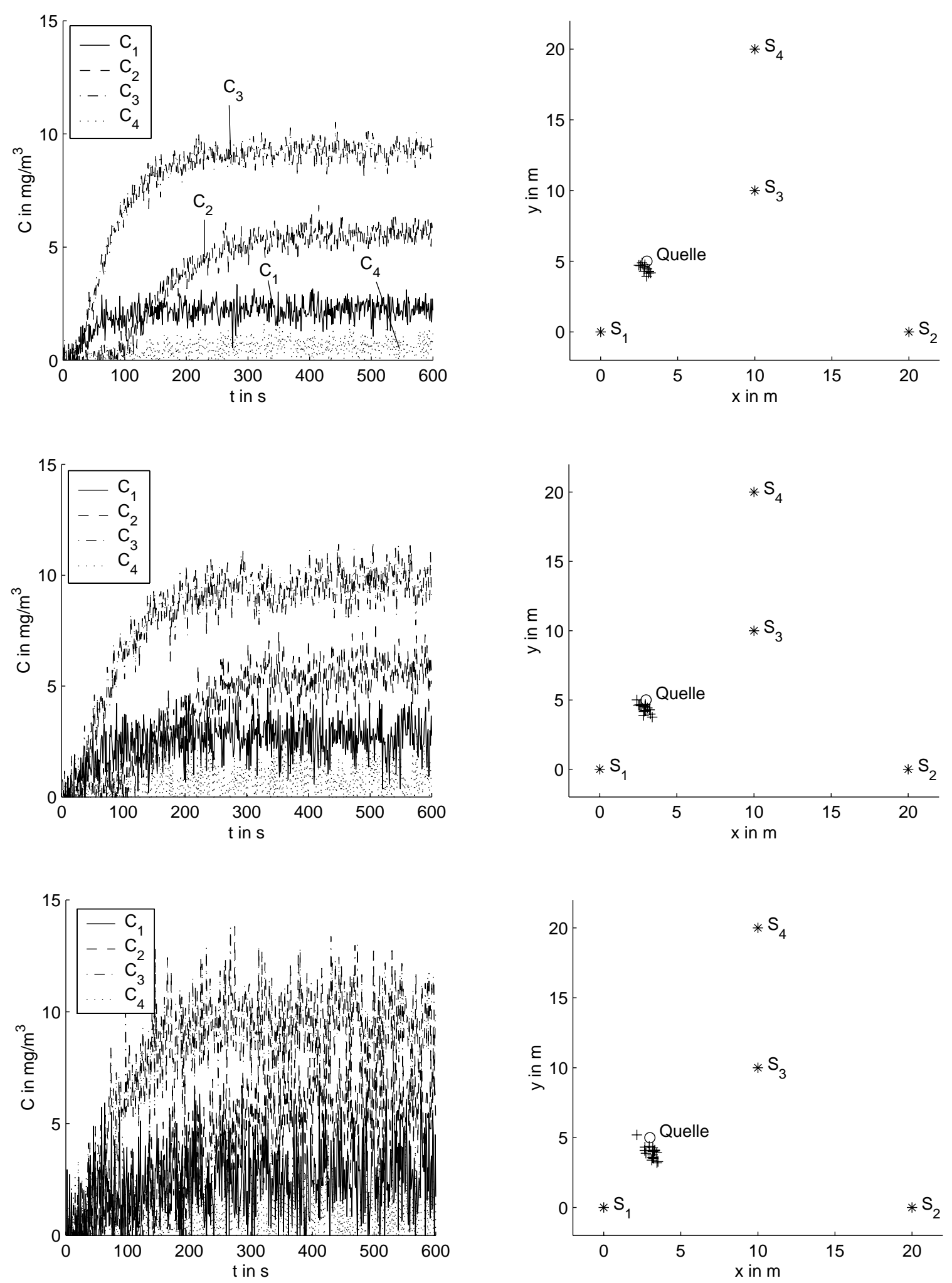

Abb. 5.4: Konzentrationsverläufe (links) sowie Quellortschätzungen für 20 Simulationen (rechts) für Quellort $\mathbf{x}_{0}=(3 \mathrm{~m}, 5 \mathrm{~m})^{T}$ und Störung mit $\pm 1.2 \mathrm{mg} / \mathrm{m}^{3}, \pm 2.4 \mathrm{mg} / \mathrm{m}^{3}$ sowie $\pm 4.8 \mathrm{mg} / \mathrm{m}^{3}$. 


\begin{tabular}{|c|c|c|c|}
\hline Quellort $\mathbf{x}_{0}^{T}$ & Störung & $\begin{array}{c}\text { Mittelwert der } \\
\text { Abweichung } \\
\left\|\hat{\mathbf{x}}_{0}-\mathbf{x}_{0}\right\|_{2}\end{array}$ & $\begin{array}{l}\text { Streuung der } \\
\text { Abweichung } \\
\left\|\hat{\mathbf{x}}_{0}-\mathbf{x}_{0}\right\|_{2}\end{array}$ \\
\hline \multirow{6}{*}{$(5 \mathrm{~m}, 10 \mathrm{~m})$} & $\begin{array}{l}\text { deterministischer } \\
\text { Kalibrierfehler }\end{array}$ & $1.80 \mathrm{~m}$ & - \\
\hline & $\begin{array}{c}\text { stochastische Abweichung der } \\
\text { Strömungsrichtung }\end{array}$ & $0.48 \mathrm{~m}$ & $0.13 \mathrm{~m}^{2}$ \\
\hline & $\begin{array}{c}\text { stochastische Abweichung der } \\
\text { Strömungsgeschwindigkeit }\end{array}$ & $0.27 \mathrm{~m}$ & $0.04 \mathrm{~m}^{2}$ \\
\hline & $\begin{array}{c}\text { alle Störungen plus Rauschen } \\
\text { mit } \pm 1.2 \mathrm{mg} / \mathrm{m}^{3}\end{array}$ & $1.83 \mathrm{~m}$ & $0.15 \mathrm{~m}^{2}$ \\
\hline & $\begin{array}{c}\text { alle Störungen plus Rauschen } \\
\text { mit } \pm 2.4 \mathrm{mg} / \mathrm{m}^{3}\end{array}$ & $1.91 \mathrm{~m}$ & $0.17 \mathrm{~m}^{2}$ \\
\hline & $\begin{array}{c}\text { alle Störungen plus Rauschen } \\
\text { mit } \pm 4.8 \mathrm{mg} / \mathrm{m}^{3} \\
\end{array}$ & $1.94 \mathrm{~m}$ & $0.18 \mathrm{~m}^{2}$ \\
\hline \multirow{6}{*}{$(3 \mathrm{~m}, 5 \mathrm{~m})$} & $\begin{array}{c}\text { deterministischer } \\
\text { Kalibrierfehler } \\
\end{array}$ & $0.91 \mathrm{~m}$ & - \\
\hline & $\begin{array}{l}\text { stochastische Abweichung der } \\
\text { Strömungsrichtung }\end{array}$ & $0.41 \mathrm{~m}$ & $0.08 \mathrm{~m}^{2}$ \\
\hline & $\begin{array}{c}\text { stochastische Abweichung der } \\
\text { Strömungsgeschwindigkeit }\end{array}$ & $0.25 \mathrm{~m}$ & $0.03 \mathrm{~m}^{2}$ \\
\hline & $\begin{array}{c}\text { alle Störungen plus Rauschen } \\
\text { mit } \pm 1.2 \mathrm{mg} / \mathrm{m}^{3}\end{array}$ & $0.91 \mathrm{~m}$ & $0.10 \mathrm{~m}^{2}$ \\
\hline & $\begin{array}{c}\text { alle Störungen plus Rauschen } \\
\text { mit } \pm 2.4 \mathrm{mg} / \mathrm{m}^{3}\end{array}$ & $0.93 \mathrm{~m}$ & $0.14 \mathrm{~m}^{2}$ \\
\hline & $\begin{array}{c}\text { alle Störungen plus Rauschen } \\
\text { mit } \pm 4.8 \mathrm{mg} / \mathrm{m}^{3}\end{array}$ & $0.94 \mathrm{~m}$ & $0.17 \mathrm{~m}^{2}$ \\
\hline
\end{tabular}

Tabelle 5.2: Mittlere Ergebnisse für jeweils 200 Simulationen pro Beispiel bei verschiedenen Störungen.

\subsection{Praktische Erprobung}

In einem industriellen Chemikalienlager für toxische Substanzen der Sigma-Aldrich-Chemie GmbH Steinheim (Deutschland) wurde für Ausbreitungsexperimente ein Netzwerk von vier Elektronischen Nasen des Typs SAMONA aufgebaut. Das SAMONA-System ist eine Entwicklung des Instituts für Instrumentelle Analytik des Forschungszentrums Karlsruhe. Es enthält sowohl ein Metalloxid-Gradienten-Array [34] als auch ein Surface-Acoustic- 
Wave-Micro-Array [91]. SAMONA besitzt zudem eine interne Anreicherungseinheit [11], die die zu messenden Substanzen über eine Zeit von 2 Minuten aufkonzentriert und anschließend den Sensor-Arrays zuführt. Damit können sehr kleine Konzentrationen gemessen werden. Zudem bewirkt die zeitliche Anreicherung eine Störungsfilterung. Allerdings werden wegen des damit verbundenen langen Messzyklus nur alle drei Minuten Messwerte von SAMONA geliefert.

Die Messungen erstreckten sich inklusive Versuchsaufbau und Vorabmessungen über eine Woche. Innerhalb dieser Zeit konnten zehn verwertbare Ausbreitungsexperimente durchgeführt werden.

Der Versuchsaufbau (Draufsicht) ist in Abb. 5.5 dargestellt. Die Sensor-Systeme befinden sich in einer Höhe von $0.9 \mathrm{~m}$. Die Hochregale sind offen und behindern die Ausbreitung nicht wesentlich. Die Strömungsrichtung ist in Abb. 5.5 durch einen Pfeil angegeben.

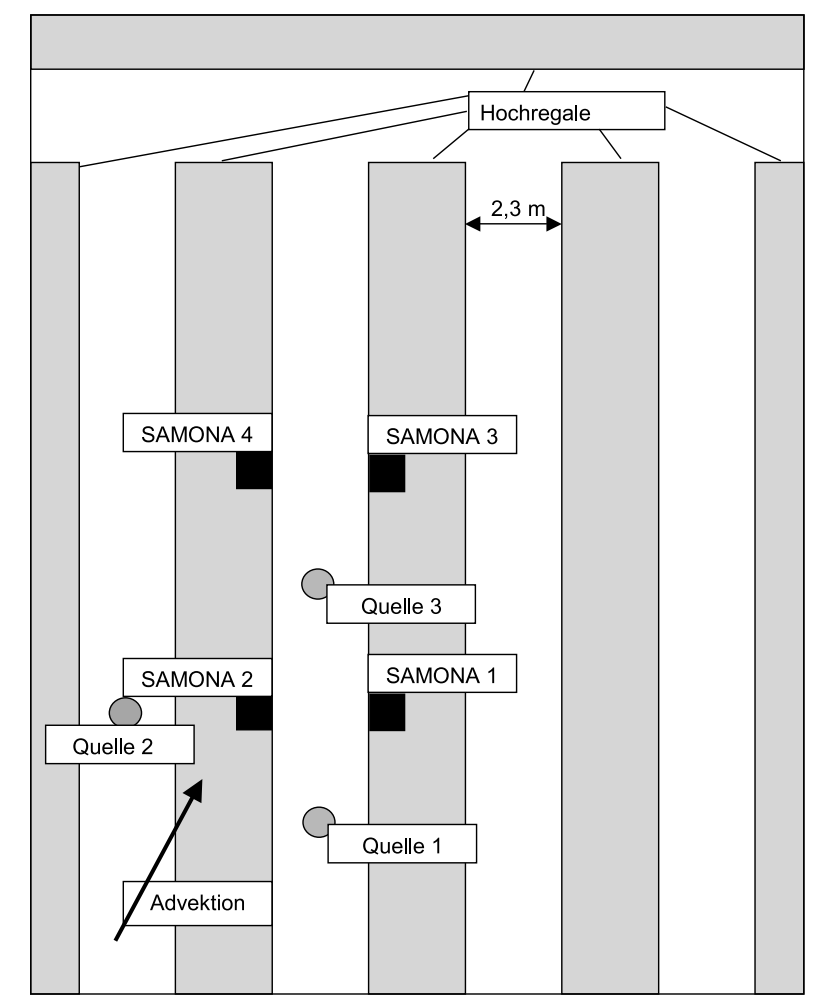

Abb. 5.5: Sensornetz mit vier SAMONA-Geräten und gewählte Quellorte (Draufsicht).

Abbildung 5.5 zeigt neben den Sensorstandorten zusätzlich die Quellorte, an denen in den Experimenten Stoffe freigesetzt wurden, um Leckagen zu simulieren. In jedem Experiment wird an einem Quellort eine bestimmte Menge einer flüssigen Substanz auf ein auf dem Boden liegendes Filterpapier geschüttet. Durch das schnelle Verdunsten wird damit eine instantane Quellintensität erhalten. Für die Quellenlokalisierung müssen die zeitlich 
integrierten Konzentrationen verwendet werden, da das Verfahren auf sprungförmigen Quellintensitäten basiert. Abbildung 5.6 zeigt die gemessenen Konzentrationsverläufe für drei Experimente sowie die zeitlich integrierten Konzentrationen.

Da sich die Sensoren nicht auf dem Boden befinden, handelt es sich hier um den idealisierten Fall einer Halbraumkonfiguration.

Die für die Quellenlokalisierung notwendigen Werte für die Strömungsgeschwindigkeit $v_{x}$ und den Diffusionskoeffizienten $K$ werden mit Hilfe zweier Vorabexperimente ermittelt. Dafür wird der Ausgangsfehler (1.1) für den in den Vorabexperimenten als bekannt vorausgesetzten Quellort bezüglich $v_{x}$ und $K$ minimiert. Dies liefert für die in den Vorabexperimenten gemessenen Konzentrationen $v_{x}=0.03 \mathrm{~m} / \mathrm{s}$ und $K=0.02 \mathrm{~m}^{2} / \mathrm{s}$.

Für die Quellenlokalisierung werden nur die stationären Endwerte der Konzentrationen benötigt. Als Schätzwert für die stationären Konzentrationen wird hier der letzte Wert der integrierten Konzentrationen jedes Sensors für jede Messung (also bei 24 min, 30 min bzw. $48 \mathrm{~min}$ ) verwendet (vgl. Abb. 5.6). Eine Signalfilterung ist wegen der Verwendung der Anreicherungseinheit nicht notwendig. Für die drei gezeigten Experimente sind die Ergebnisse der Quellenlokalisierung in Abb. 5.7 dargestellt. Dabei wurde das Koordinatensystem in die Strömungsrichtung gedreht. In Abb. 5.7 (rechts) ist die Menge $\mathcal{P}_{i}$ (Oval) für jeden Sensor für optimales $q_{0}$ dargestellt. Nachfolgend werden die Ergebnisse der gezeigten drei Experimente diskutiert.

\section{Experiment 1}

Aufgrund der Lage von Quellort 1 und der Strömungsrichtung ist zu erwarten, dass Sensor $S_{1}$ von Beginn an die größte Konzentration misst. Aufgrund von Störungen in der Ausbreitung (z. B. leicht wechselnde Strömungsrichtung) kommt es dazu, dass Sensor $S_{2}$ anfänglich höhere Konzentrationen misst, wodurch die zeitlich integrierten Konzentrationen von Sensor $S_{2}$ bis ca. 16 Minuten nach Emissionsbeginn größer sind als die von Sensor $S_{1}$. Dadurch erreicht der stationäre Endwert von Sensor $S_{1}$ einen kleineren Wert als in Bezug auf $S_{2}$ erwartet (nahezu gleich wie $S_{2}$ ). Dies führt zu einem geschätzten Quellort, in dem eine Quelle unter Berücksichtigung der identifizierten Strömungsrichtung bei beiden Sensoren $S_{1}$ und $S_{2}$ annähernd gleiche Konzentrationen hervorruft. Die Abweichung zum tatsächlichen Quellort beträgt ca. 1.3m. Die Abweichung lässt sich auch anhand der Mengen $\mathcal{P}_{i}$ (Ovale) verdeutlichen. Durch die zu geringe Konzentration bei $S_{1}$ wird ein zu großes Oval für diesen Sensor erhalten. Mit einer größeren Konzentration und damit einem kleineren Oval für $S_{1}$ liegt der geschätzte Quellort näher am tatsächlichen Quellort. 


\section{Experiment 2}

In Experiment 2 unterliegen die Konzentrationsmesswerte großen Schwankungen. Dennoch ergibt sich für die integrierten Konzentrationen ein nahezu erwartungsgemäßer Verlauf. Allein die Konzentration am Sensor $S_{2}$ ist kleiner als erwartet, nämlich etwa nur so groß wie die Konzentration am Sensor $S_{1}$. Dies ist wiederum auf Störungen bei der Ausbreitung zurückzuführen. Eine größere Konzentration bei Sensor $S_{2}$ wird den geschätzten Quellort näher in Richtung $S_{2}$ bewegen und damit die Abweichung von 0.9m zwischen geschätztem und tatsächlichem Quellort verringern. Dies wird erneut bei Betrachtung der Mengen $\mathcal{P}_{i}$ deutlich. Eine größere Konzentration und damit ein kleineres Oval für $S_{2}$ verschiebt den geschätzten Quellort in Richtung tatsächlichen Quellort.

\section{Experiment 3}

Die Konzentrationsmesswerte bei Experiment 3 und damit auch die Verläufe der integrierten Konzentrationen sehen sehr gut aus. Die in Strömungsrichtung liegenden Sensoren $S_{3}$ und $S_{4}$ messen größere Konzentrationen als die Sensoren $S_{1}$ und $S_{2}$. Jedoch sind die Konzentrationen bei $S_{1}$ und $S_{2}$ unter Berücksichtigung der identifizierten Strömungsgeschwindigkeit größer als erwartet. Dies kann durch eine temporär niedrigere Strömungsgeschwindigkeit als die identifizierte erklärt werden. Aus diesem Grund wird der geschätzte Quellort in Richtung der Sensoren $S_{1}$ und $S_{2}$ verschoben und weicht somit um ca. $1.3 \mathrm{~m}$ vom tatsächlichen Quellort ab.

Zusammenfassend lässt sich feststellen, dass in den drei gezeigten Experimenten die Schätzwerte für die Quellorte nicht direkt auf den wahren Quellorten liegen. Das ist auf Kalibrierfehler und schwankende Strömungsverhältnisse zurückzuführen. Dennoch stellen die geschätzten Quellorte unter Berücksichtigung dieser Störeinflüsse befriedigende Näherungen für die tatsächlichen Quellorte dar.

In drei weiteren Experimenten konnten ebenfalls vergleichbar gute Resultate erzielt werden, wobei eine veränderte Strömungsgeschwindigkeit berücksichtigt werden musste. Diese Veränderung wurde infolge schlechter Quellortschätzungen erkannt, die sich durch das Verwenden der vorab identifizierten Strömungsgeschwindigkeit ergaben. Das Erkennen von veränderten Ausbreitungsbedingungen ist aber im praktischen Einsatz nicht möglich. Als Ausweg bietet sich der Einsatz von Anemometern zur Online-Messung der Strömungsrichtung und -geschwindigkeit an. 

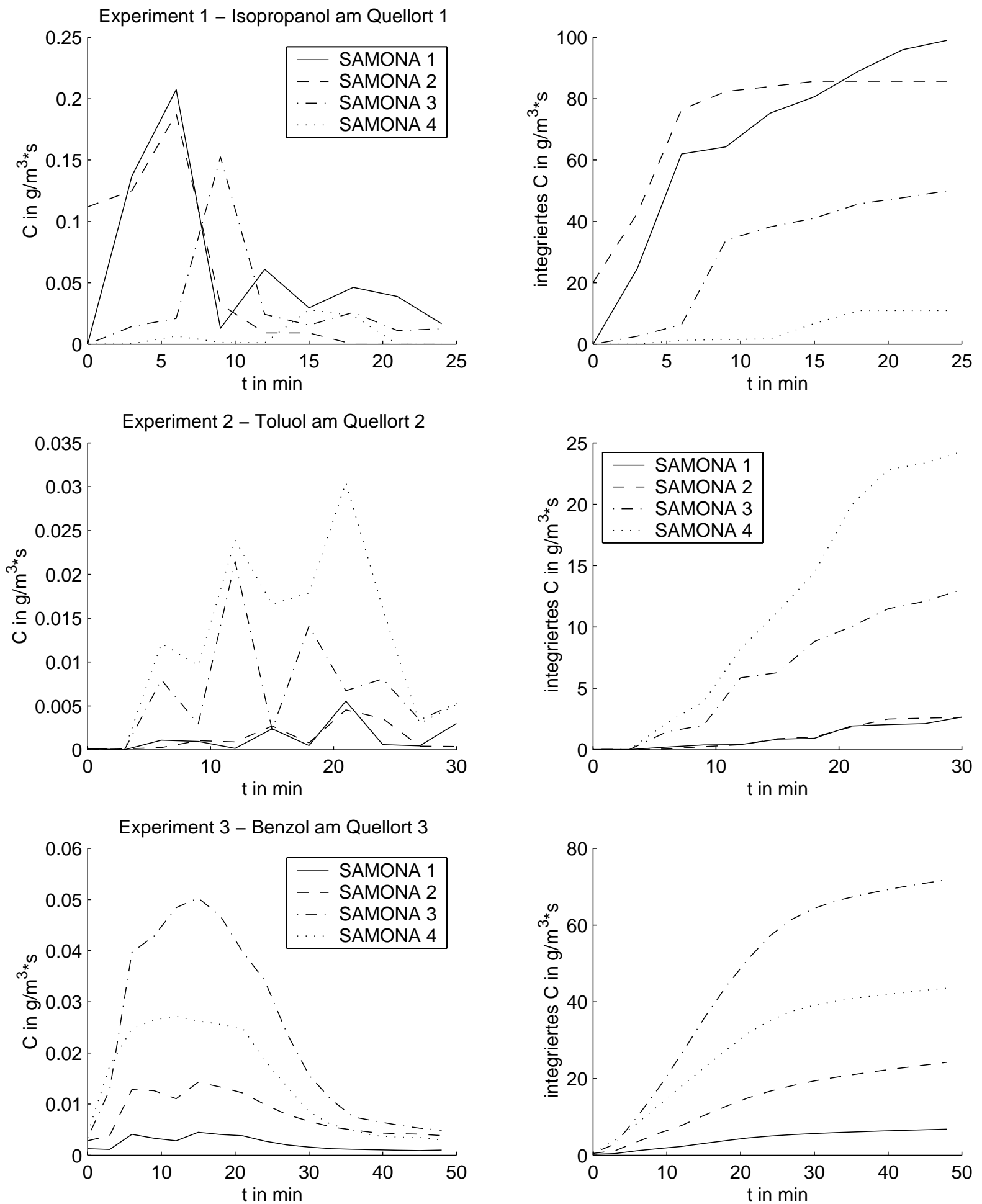

Abb. 5.6: Gemessene und zeitlich integrierte Konzentrationen für drei Experimente. 

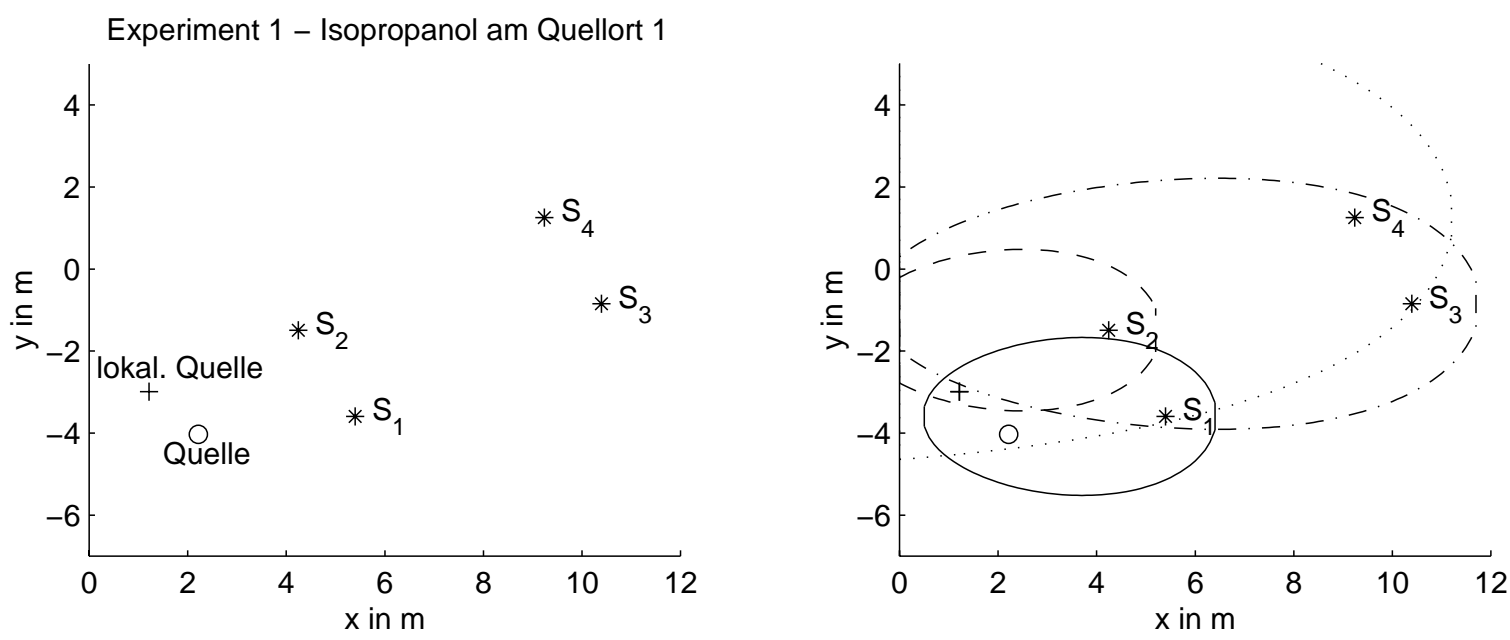

Experiment 2 - Toluol am Quellort 2
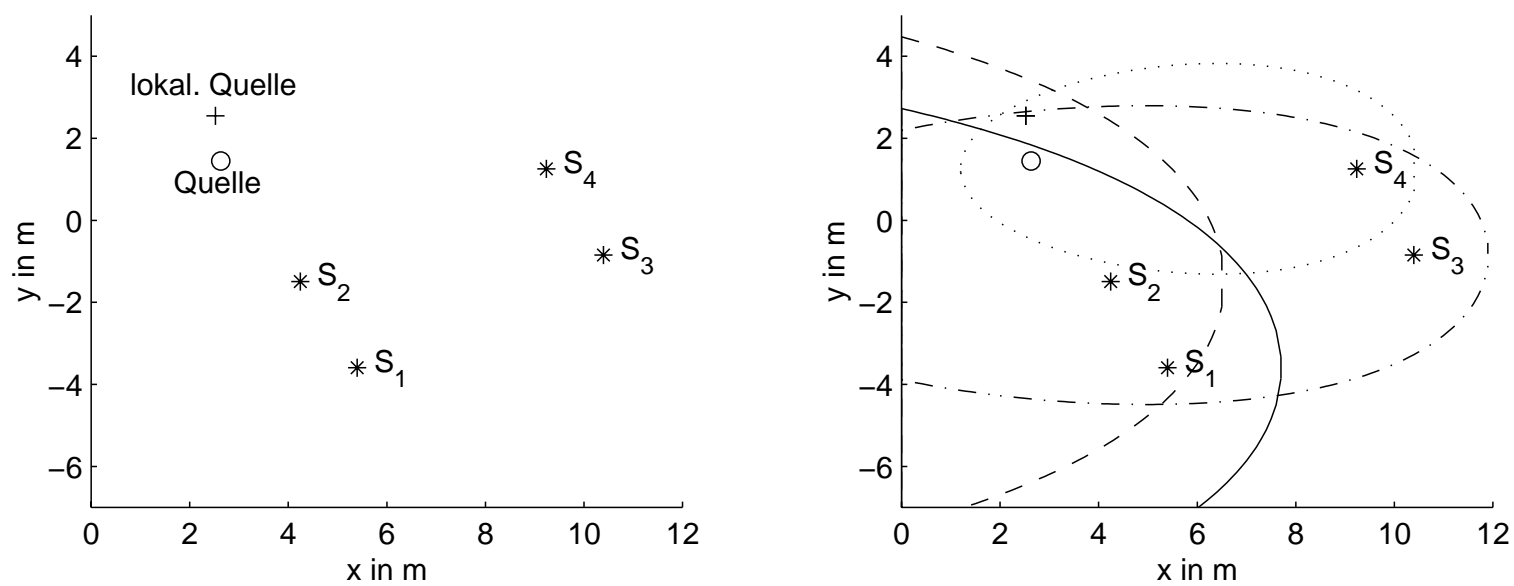

Experiment 3 - Benzol am Quellort 3
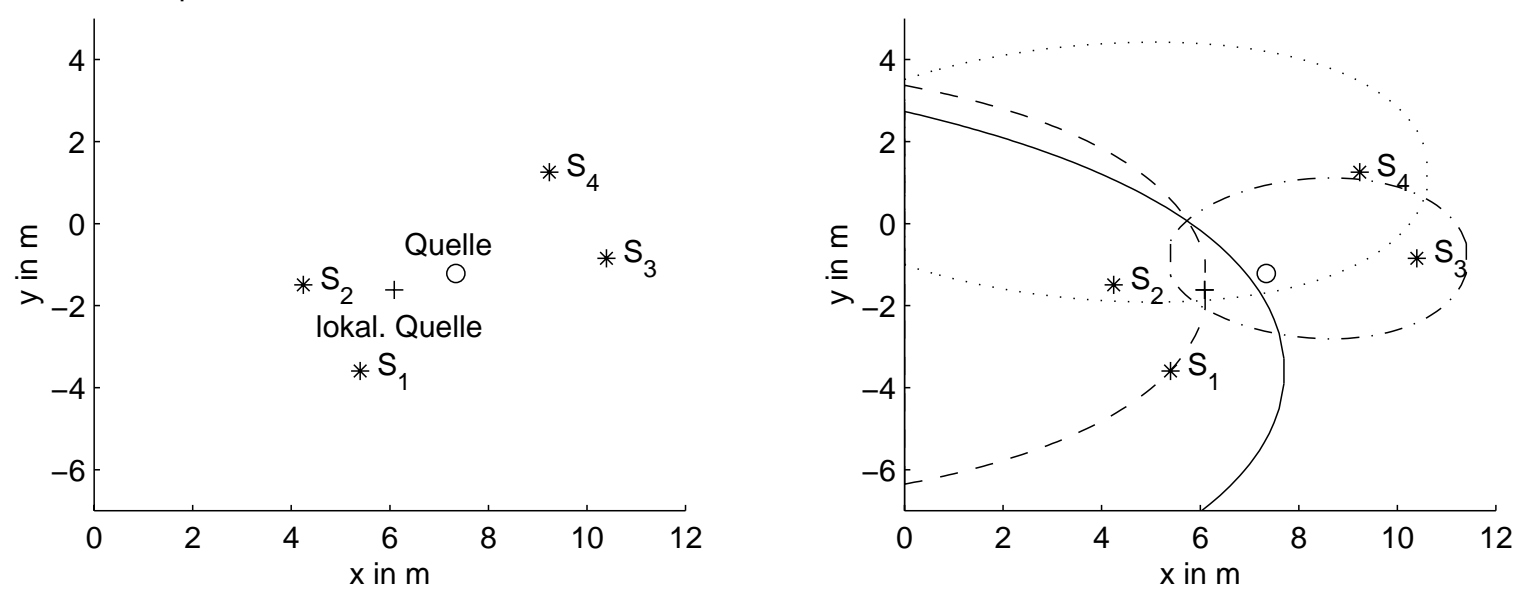

Abb. 5.7: Lage der lokalisierten Quellorte $(+)$ sowie der tatsächlichen Quellorte (o) für drei Experimente. Rechts sind die Mengen $\mathcal{P}_{i}$ (Ovale) für alle vier Sensoren dargestellt. 
Zwei abschließende Experimente ergaben keine sinnvollen Schätzwerte für die Quellorte. Die zugehörigen gemessenen Konzentrationsverläufe ließen sich nicht mit einem DiffusionsAdvektions-Modell erklären. Die Konzentrationsmessungen eines dieser Beispiele (Experiment 7) zeigt Abb. 5.8.
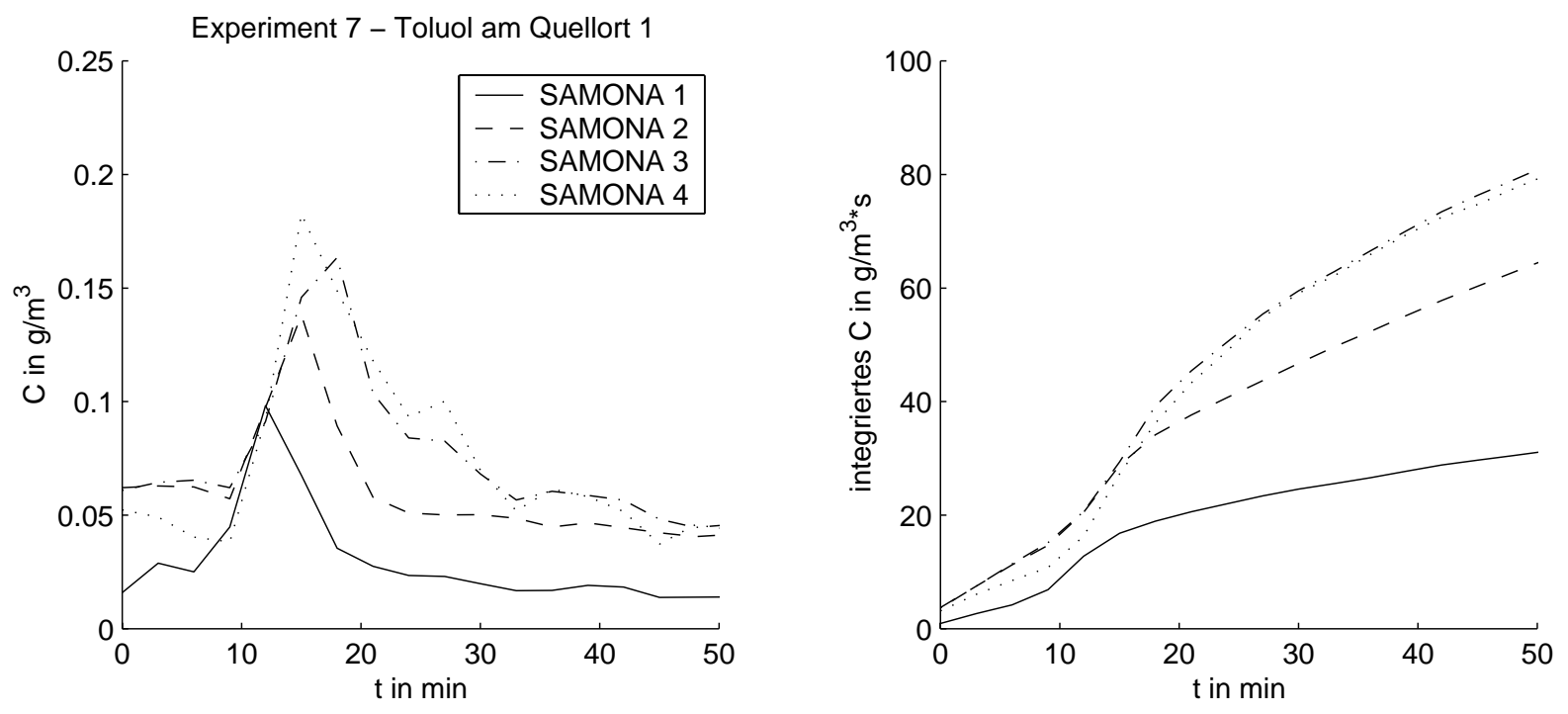

Abb. 5.8: Gemessene und zeitlich integrierte Konzentrationen für Experiment 7.

Aufgrund der Lage der Sensoren und der Quelle am Quellort 1 müssen bei einer Ausbreitung durch Diffusion-Advektion die Sensoren $S_{1}$ und $S_{2}$ größere Konzentrationen messen als die Sensoren $S_{3}$ und $S_{4}$, egal ob und in welcher Richtung eine Strömung erfolgt. Dies ist in Experiment 7 nicht der Fall; die Sensoren $S_{3}$ und $S_{4}$ messen gleich große integrierte Konzentrationen, die größer sind als die von $S_{2}$ und $S_{1}$.

Die Konzentrationsverläufe in Abb. 5.8 lassen sich nur auf erhebliche Verwirbelungen im Ausbreitungsgebiet zurückführen, die durch vorbei fahrende Gabelstapler und sich bewegende Personen hervorgerufen wurden. Aus diesen Experimenten wird klar, dass die modellbasierte Quellenlokalisierung nur dann möglich ist, wenn keine großen Störungen durch bewegte Objekte usw. vorliegen. Da praktisch eine automatische Leckagedetektion und Quellenlokalisierung gerade dann wichtig ist, wenn keine Kontrolle durch Personen im zu überwachendem Gebiet erfolgt (z. B. nachts), schränkt dieses Forderung die Anwendbarkeit der modellbasierten Verfahren nicht wesentlich ein. 


\section{Zusammenfassung und Ausblick}

Das Ziel der vorliegenden Arbeit bestand darin, neue Verfahren zur Lokalisierung von Emissionsquellen auf der Basis räumlich verteilter Konzentrationsmessungen zu entwickeln. Ausgehend von einem geometrischen Zugang wurden neue, zweistufige Verfahren zur Quellenlokalisierung sowohl für den Fall der Ausbreitung durch reine Diffusion als auch für den Fall von Diffusion-Advektion entwickelt.

Grundidee des geometrischen Zugangs ist es, zunächst für jeden Sensor die Menge aller Punkte zu bestimmen, auf der sich aus der Sicht des einzelnen Sensors die Quelle befinden kann. Durch Bilden der Schnittmenge für mehrere Sensoren lässt sich dann daraus der Quellort ermitteln. Die entwickelten Verfahren umgehen die numerischen Schwierigkeiten der klassischen ausgangsfehlerbasierten einstufigen Verfahren (Maximum-likelihoodSchätzung). Zudem liefert der Zugang Aussagen zur Existenz und Eindeutigkeit von Lösungen, zur Lage von Mehrfachlösungen sowie zur geeigneten Platzierung der Sensoren innerhalb vorgegebener Bereiche für die Quellenlokalisierung.

Für die Entwicklung der neuen Verfahren wurden im Kapitel 2 Ausbreitungsmodelle in Form von inhomogenen Diffusions-Advektions-Gleichungen für den Fall reiner Diffusion sowie für den Fall von Diffusion-Advektion hergeleitet. Für das halbunendliche und unendliche sowie für das endliche Ausbreitungsmedium wurden mittels der Greenschen Funktion geschlossene Lösungen erarbeitet. Durch Messungen wurde gezeigt, dass die Modellierung auf der Basis der Diffusions-Advektions-Gleichung praktisch sinnvoll ist.

In Kapitel 3 wurde der geometrische Zugang genutzt, um eine neues, zweistufiges Verfahren zur Quellenlokalisierung für den Fall reiner Diffusion zu entwickeln. Dabei wurde zunächst der ungestörte Fall mit minimaler Sensoranzahl untersucht, um grundlegende Aussagen zur Existenz und Eindeutigkeit sowie zur Lage von Lösungen des Quellenlokalisierungsproblems herauszuarbeiten. Anschließend wurde der gestörte Fall mit beliebiger Sensoranzahl behandelt. 
In Kapitel 4 wurden die Verfahren zur Quellenlokalisierung auf den Fall eines zusätzlichen homogenen Strömungsfelds (Advektion) erweitert. Analog zum Fall reiner Diffusion wurde erst der ungestörte Fall mit minimaler Sensoranzahl und dann der gestörte Fall mit beliebiger Sensoranzahl untersucht.

Kapitel 5 beschäftigte sich mit der simulativen und praktischen Erprobung der neuen Verfahren. Anhand von zahlreichen Simulationen wurden die wesentlichen statistischen Eigenschaften der neuen Verfahren herausgearbeitet. Abschließend wurden die Ergebnisse experimenteller Untersuchungen in einem industriellen Chemikalienlager vorgestellt.

Die wichtigsten Ergebnisse der Arbeit sind:

1. Entwicklung neuer, zweistufiger Verfahren zur Quellenlokalisierung für Diffusionsund Diffusions-Advektions-Vorgänge auf der Basis räumlich verteilter, punktweiser Konzentrationsmessungen.

2. Einführung so genannter skalierbarer Sensor-Quell-Abstände für den Diffusionsfall sowie verallgemeinerter skalierbarer Sensor-Quell-Abstände im Fall von DiffusionAdvektion.

3. Ableitung von Algorithmen zur Schätzung skalierbarer Sensor-Quell-Abstände sowohl auf der Basis stationärer als auch instationärer Konzentrationen im ersten Schritt der zweistufigen Verfahren.

4. Herleitung von Algorithmen zur Ermittlung des Quellorts auf der Basis skalierbarer Sensor-Quell-Abstände für den ungestörten und gestörten Fall bei beliebiger Sensoranzahl im zweiten Schritt der zweistufigen Verfahren.

5. Nachweis, dass bei Diffusion im ungestörten Fall mit minimaler Sensoranzahl neben der wahren stets eine scheinbare Lösung existiert, die sich durch einen zusätzlichen Sensor ausschließen lässt, wenn dieser nicht auf der Umkugel der anderen Sensoren liegt.

6. Nachweis, dass bei Diffusion-Advektion mit minimaler Sensoranzahl abhängig vom Quellort neben der wahren auch eine scheinbare Lösung existieren kann, die sich stets durch einen zusätzlichen Sensor innerhalb der konvexen Hülle der anderen Sensoren ausschließen lässt.

7. Entwicklung einer approximativ-optimalen Wichtungsstrategie, die die Streuung der Quellortschätzungen bei gestörten Konzentrationsmessungen wesentlich verringert 
und damit die gleiche Schätzgüte wie einstufige ausgangsfehlerbasierte Verfahren erreicht, ohne deren numerische Schwierigkeiten zu besitzen.

8. Implementierung der neuen Verfahren in ein Simulationssystem und breitgefächerte Untersuchungen anhand von Komplexbeispielen (mehr als 3600 Simulationen).

9. Erfolgreiche praktische Erprobung der neuen Verfahren in einem industriellen Chemikalienlager.

Durch zukünftige Arbeiten ist zu prüfen, wie sich der neue, geometrische Zugang für einund zweidimensionale Ausbreitungsmedien sowie komplexere Ausbreitungsszenarien und Raumgeometrien (z. B. inhomogener Medien) nutzen lässt.

Ein weiterer Aspekt ist die Untersuchung der potenziellen Leistungsfähigkeit hybrider Sensornetze, die sowohl aus stationären als auch aus mobilen Sensoren bestehen. Zur hybriden Quellenlokalisierung sind bisher keine Algorithmen bekannt.

Nicht zuletzt ist eine rechentechnische Umsetzung der neuen Algorithmen in ein Sensormanagementsystem wie beispielsweise INSPECT-S Voraussetzung für eine industrielle Nutzung der entwickelten Verfahren zur Quellenlokalisierung. 


\section{A Elektronische Nasen}

Die chemische Erkennungsebene besteht aus einem Array von sensitiven Rezeptoren (Sensor-Array). Jeder Sensor tritt selektiv mit dem Analyten in Wechselwirkung, wodurch seine physikalischen Eigenschaften verändert werden. Diese Änderung wird durch den Transducer in ein elektrisch verwertbares Signal umgewandelt. Die Signale des gesamten Sensor-Arrays liefern somit ein geruchsspezifisches Signalmuster, das anschließend durch eine Datenverarbeitung in der numerischen Erkennungsebene klassifiziert wird. Die messbaren Parameter sind in Abhängigkeit vom Sensortyp z. B. Temperatur, Masse oder elektrische Kenngrößen, wie Spannung, Strom, Leitfähigkeit und Kapazität. Die wichtigsten Sensortypen und deren Wirkprinzipien sind nachfolgend beschrieben.

- Ein Metall-Oxid-Sensor besteht aus einem halbleitenden Metalloxid (meist Zinndioxid), das zusätzlich eine selektiv permeable Keramikmembran besitzen kann. Wird der Sensor auf eine Betriebstemperatur zwischen 100 bis $500^{\circ} \mathrm{C}$ aufgeheizt, dann adsorbiert das Metalloxid Sauerstoff aus der Umgebungsluft, der an der Oberfläche reduziert wird. Dies führt zu einer Herabsetzung der Elektronenkonzentration und damit der Leitfähigkeit des Halbleiters. Reduzierende Gase reagieren mit dem an der Oberfläche adsorbierten Sauerstoff und führen damit zu einer Erhöhung der Leitfähigkeit. Die Leitfähigkeitsänderung hängt dabei von der Beschichtung sowie von der Betriebstemperatur des Sensors ab. Werden die Sensoren des Sensor-Arrays mit verschiedenen Beschichtungen und/oder mit verschiedenen Temperaturen betrieben, so liefern alle Leitfähigkeitsänderungen des Arrays ein geruchsspezifisches Muster, das durch die numerische Erkennungsebene klassifiziert werden kann. Ein Metall-Oxid-Sensor-System ist beispielsweise die Karlsruher Mikronase KAMINA des Forschungszentrums Karlsruhe [4, 5, 27, 34, 35, 75]. Weitere Systeme und deren Anwendung sind z. B. in $[56,57,106]$ zu finden.

- Ein Leitendes-Polymer-Sensor [8, 43, 46] besteht im Wesentlichen aus einer elektrisch leitenden Polymerschicht. Bei Kontakt mit bestimmten Analyten ändert sich 
in Abhängigkeit von der Art des Polymers deren Leitwert. Wird das Sensor-Array aus Sensoren verschiedener Polymere aufgebaut, liefern die Leitwertänderungen ein geruchsspezifisches Muster.

- Ein Feld-Effekt-Sensor [78, 109] besteht aus einem Feld-Effekt-Transistor, der zusätzlich eine Isolatorschicht am Gate besitzt. Die mit Feld-Effekt-Sensoren analysierbaren Moleküle $\mathrm{H}_{2}, \mathrm{H}_{2} \mathrm{~S}, \mathrm{C}_{2} \mathrm{H}_{5} \mathrm{OH}$ geben Wasserstoffatome an eine Metallschicht $(P d, P t, I r)$ ab, die dann eine Dipol-Schicht in der Isolatorschicht bilden. Diese verursacht eine messbare Spannungsänderung am Sensor. Die Eigenschaften des Sensors lassen sich durch Variieren von Struktur und Dicke der Metallschicht beeinflussen.

- Ein Schwingquarz-Sensor [7, 38, 44] besteht aus einem dünnen Quarzplättchen, auf dessen gegenüberliegenden Flächen zwei Elektroden aufgebracht sind. Wird an diese Elektroden eine Spannung angelegt, so kommt es auf Grund der Elektrostriktion - der reziproke Effekt zum piezoelektrischen Effekt - zu einer mechanischen Deformation des Quarzes. Durch Anlegen einer hochfrequenten Wechselspannung bilden sich stehende Wellen aus. Die Resonanzfrequenz ist dabei der Dicke des Plättchens umgekehrt proportional. Der Schwingquarz wird mit einer chemisch sensitiven Schicht versehen, in die sich je nach Beschichtungsmaterial bestimmte Analyten einlagern. Diese Einlagerung führt zu einer Vergrößerung der Dicke des Schwingquarzes und damit zu einer messbaren Änderung der Resonanzfrequenz. In einer Elektronischen Nase werden Schwingquarze mit unterschiedlicher sensitiver Beschichtung eingesetzt, um ein geruchsspezifisches Muster von Frequenzänderungen zu erhalten.

- Ein Surface-Acoustic-Wave-Sensor besteht aus so genannten interdigital transducers (IDT), d.h. aus kammartig ineinander greifenden Elektroden, die mittels Photolithographie auf ein Substrat aufgebracht werden. Durch Anlegen einer hochfrequenten Wechselspannung wird eine Oberflächenwelle in den IDTs erzeugt, deren Resonanzfrequenz von der Bauweise der IDTs sowie von der Schallgeschwindigkeit abhängt und bis zu $2 \mathrm{GHz}$ erreichen kann. Wie bei den Schwingquarzen kann durch chemisch sensitive Schichten eine Einlagerung von bestimmten Analyten erzielt werden, die hier eine Änderung der Schallgeschwindigkeit und damit eine messbare Änderung der Resonanzfrequenz bewirkt. Ein Surface-Acoustic-WaveSensorsystem ist beispielsweise das SAGAS-System des Forschungszentrums Kalrsruhe $[11,91,92,93]$. Weitere Systeme und deren Anwendung sind in [87, 98, 113] zu finden. 
Die numerische Erkennungsebene muss anhand der vom Sensor-Array gelieferten Signalmuster den aktuellen Geruch klassifizieren und wenn möglich eine quantitative Analyse (Konzentrationsbestimmung) durchführen. Dazu müssen in einer Trainingsphase Signalmuster für die zu klassifizierenden und quantifizierenden Gerüche generiert werden, mit denen die Auswertealgorithmen angelernt werden. In der Anwendungsphase der Elektronischen Nase können damit nur die vorab gelernten Gerüche wiedererkannt und quantifiziert werden. Für die Klassifikation kommen zum einen statistische Verfahren, wie Hauptkomponenten- und Diskriminanzanalyse [78, 82, 87, 100, 108, 113], als auch Neuronale Netze $[12,17,47,49,67,80,89,105,106]$ zum Einsatz. Die quantitative Analyse kann ebenfalls durch Neuronale Netze erfolgen. Oft werden jedoch Regressionsmodelle verwendet, deren Parameter meist mit dem Partial-Least-Squares (PLS) Algorithmus [23, 112] ermittelt werden.

Anwendungen Elektronischer Nasen als Einzelsysteme finden sich beispielsweise bei

- der Qualitätsüberwachung in der Lebensmittelindustrie [16, 77, 85, 86, 98, 108],

- der medizinische Diagnose durch Analyse des Atems [25, 31, 66, 82, 110],

- der Kontrolle des Innenraumgeruchs von Kraftfahrzeugen [32, 43, 78, 79] und

- der Luftüberwachung in Raumstationen [114]. 


\section{B Nachweis der Greenschen}

\section{Funktionen}

Nachfolgend wird nachgewiesen, dass die GreEnsche Funktion (2.19)

$$
C_{G}(\mathbf{x}, t)=\left(\frac{1}{2 \sqrt{\pi K\left(t-t_{0}\right)}}\right)^{n} \cdot \exp \left(-\frac{\left\|\mathbf{x}-\mathbf{x}_{0}\right\|_{2}^{2}}{4 K\left(t-t_{0}\right)}\right) \cdot 1\left(t-t_{0}\right)
$$

die zugehörige partielle Differenzialgleichung erfüllt. Dies wird hier für den dreidimensionalen Fall durchgeführt. Der Nachweis lässt sich aber analog auch für den zwei- bzw. eindimensionalen Fall zeigen.

Für $n=3$ lautet (2.19)

$$
C_{G}(\mathbf{x}, t)=\left(\frac{1}{2 \sqrt{\pi K\left(t-t_{0}\right)}}\right)^{3} \cdot \exp \left(-\frac{\left\|\mathbf{x}-\mathbf{x}_{0}\right\|_{2}^{2}}{4 K\left(t-t_{0}\right)}\right) \cdot 1\left(t-t_{0}\right)
$$

mit $\mathbf{x}=(x, y, z)^{T}, \mathbf{x}_{0}=\left(x_{0}, y_{0}, z_{0}\right)^{T}$. (B.2) soll die partielle Differenzialgleichung (2.17)

$$
\frac{\partial C_{G}}{\partial t}-K \frac{\partial^{2} C_{G}}{\partial x^{2}}-K \frac{\partial^{2} C_{G}}{\partial y^{2}}-K \frac{\partial^{2} C_{G}}{\partial z^{2}}=\delta\left(t-t_{0}\right) \cdot \delta\left(x-x_{0}\right) \cdot \delta\left(y-y_{0}\right) \cdot \delta\left(z-z_{0}\right)
$$

erfüllen.

Dazu werden nachfolgend die zeitlichen und örtlichen Ableitungen von (B.2) gebildet.

Mit Hilfe der Produktregel lautet die zeitliche Ableitung

$$
\begin{aligned}
\frac{\partial C_{G}}{\partial t}= & 1\left(t-t_{0}\right) \\
& \times\left[\left(\frac{1}{2 \sqrt{\pi K\left(t-t_{0}\right)}}\right)^{2} \cdot \frac{1}{2 \sqrt{\pi K\left(t-t_{0}\right)}} \cdot\left(-\frac{3}{2\left(t-t_{0}\right)}\right) \cdot \exp \left(-\frac{\left\|\mathbf{x}-\mathbf{x}_{0}\right\|_{2}^{2}}{4 K\left(t-t_{0}\right)}\right)\right. \\
& \left.+\left(\frac{1}{2 \sqrt{\pi K\left(t-t_{0}\right)}}\right)^{3} \cdot \exp \left(-\frac{\left\|\mathbf{x}-\mathbf{x}_{0}\right\|_{2}^{2}}{4 K\left(t-t_{0}\right)}\right) \cdot\left(-\frac{\left\|\mathbf{x}-\mathbf{x}_{0}\right\|_{2}^{2}}{4 K}\right) \cdot\left(-\frac{1}{\left(t-t_{0}\right)^{2}}\right)\right] \\
& +\left(\frac{1}{2 \sqrt{\pi K\left(t-t_{0}\right)}}\right)^{3} \cdot \exp \left(-\frac{\left\|\mathbf{x}-\mathbf{x}_{0}\right\|_{2}^{2}}{4 K\left(t-t_{0}\right)}\right) \cdot \delta\left(t-t_{0}\right)
\end{aligned}
$$


bzw.

$$
\begin{aligned}
\frac{\partial C_{G}}{\partial t}= & C_{G}(\mathbf{x}, t) \cdot\left[\left(-\frac{3}{2\left(t-t_{0}\right)}\right)+\left(\frac{\left\|\mathbf{x}-\mathbf{x}_{0}\right\|_{2}^{2}}{4 K\left(t-t_{0}\right)^{2}}\right)\right] \\
& +\left(\frac{1}{2 \sqrt{\pi K\left(t-t_{0}\right)}}\right)^{3} \cdot \exp \left(-\frac{\left\|\mathbf{x}-\mathbf{x}_{0}\right\|_{2}^{2}}{4 K\left(t-t_{0}\right)}\right) \cdot \delta\left(t-t_{0}\right) .
\end{aligned}
$$

Die ersten und zweiten Ableitungen nach der Ortskoordinate $x$ lauten

$$
\begin{aligned}
\frac{\partial C_{G}}{\partial x} & =\left(\frac{1}{2 \sqrt{\pi K\left(t-t_{0}\right)}}\right)^{3} \cdot \exp \left(-\frac{\left\|\mathbf{x}-\mathbf{x}_{0}\right\|_{2}^{2}}{4 K\left(t-t_{0}\right)}\right) \cdot 1\left(t-t_{0}\right) \cdot\left(-\frac{x-x_{0}}{2 K\left(t-t_{0}\right)}\right) \\
& =C_{G}(\mathbf{x}, t) \cdot\left(-\frac{x-x_{0}}{2 K\left(t-t_{0}\right)}\right)
\end{aligned}
$$

und

$$
\frac{\partial^{2} C_{G}}{\partial x^{2}}=C_{G}(\mathbf{x}, t) \cdot \frac{1}{K} \cdot\left[\left(\frac{\left(x-x_{0}\right)^{2}}{4 K\left(t-t_{0}\right)^{2}}\right)-\frac{1}{2\left(t-t_{0}\right)}\right] .
$$

Die Ableitungen nach $y$ und $z$ werden durch Ersetzen von $x, x_{0}$ durch $y, y_{0}$ bzw. $z, z_{0}$ in (B.6) und (B.7) erhalten.

Mit der Differenzialgleichung (2.17) entsteht die Forderung

$$
\begin{gathered}
C_{G}(\mathbf{x}, t) \cdot\left[\frac{\left(-\frac{3}{2\left(t-t_{0}\right)}\right)}{\left(\frac{\left\|\mathbf{x}-\mathbf{x}_{0}\right\|_{2}^{2}}{4 K\left(t-t_{0}\right)^{2}}\right)}\right] \\
+\left(\frac{1}{2 \sqrt{\pi K\left(t-t_{0}\right)}}\right)^{3} \cdot \exp \left(-\frac{\left\|\mathbf{x}-\mathbf{x}_{0}\right\|_{2}^{2}}{4 K\left(t-t_{0}\right)}\right) \cdot \delta\left(t-t_{0}\right) \\
-C_{G}(\mathbf{x}, t) \cdot\left[\frac{\left(\frac{\left(x-x_{0}\right)^{2}}{4 K\left(t-t_{0}\right)^{2}}\right)+\left(\frac{\left(y-y_{0}\right)^{2}}{4 K\left(t-t_{0}\right)^{2}}\right)+\left(\frac{\left(z-z_{0}\right)^{2}}{4 K\left(t-t_{0}\right)^{2}}\right)}{\left(\frac{!}{=} \delta\left(t-t_{0}\right) \cdot \delta\left(x-x_{0}\right) \cdot \delta\left(y-y_{0}\right) \cdot \delta\left(z-z_{0}\right)\right.}-\left(\frac{3}{2\left(t-t_{0}\right)}\right)\right]
\end{gathered}
$$

und durch Kürzen der ober- bzw. unterstrichenen Terme

$$
\begin{array}{r}
\left(\frac{1}{2 \sqrt{\pi K\left(t-t_{0}\right)}}\right)^{3} \cdot \exp \left(-\frac{\left\|\mathbf{x}-\mathbf{x}_{0}\right\|_{2}^{2}}{4 K\left(t-t_{0}\right)}\right) \cdot \delta\left(t-t_{0}\right) \\
\stackrel{!}{=} \delta\left(t-t_{0}\right) \cdot \delta\left(x-x_{0}\right) \cdot \delta\left(y-y_{0}\right) \cdot \delta\left(z-z_{0}\right)
\end{array}
$$

Diese Gleichung wegen $\delta\left(t-t_{0}\right)=0$ für $t-t_{0} \neq 0$ stets erfüllt. Es muss also nur noch gezeigt werden, dass die folgende Gleichung für $t \rightarrow t_{0}$ gilt

$$
\begin{array}{r}
\left(\frac{1}{2 \sqrt{\pi K\left(t-t_{0}\right)}}\right)^{3} \cdot \exp \left(-\frac{\left(x-x_{0}\right)^{2}+\left(y-y_{0}\right)^{2}+\left(z-z_{0}\right)^{2}}{4 K\left(t-t_{0}\right)}\right) \\
\stackrel{!}{=} \delta\left(x-x_{0}\right) \cdot \delta\left(y-y_{0}\right) \cdot \delta\left(z-z_{0}\right) .
\end{array}
$$


Wie bereits erwähnt, ist die $\delta$-Funktion keine Funktion im klassischen Sinne, sondern eine singuläre verallgemeinerte Funktion [33, 101]. Zwei verallgemeinerte Funktionen $f_{1}$ und $f_{2}$ werden als gleich angesehen, wenn ihre zugeordneten Funktionale übereinstimmen

$$
\left\langle f_{1}, \varphi\right\rangle \equiv\left\langle f_{2}, \varphi\right\rangle \quad \text { für alle Testfunktionen } \varphi \text {. }
$$

Für das der $\delta$-Funktion zugeordnete Funktional gilt ${ }^{1}$

$$
\left\langle\delta\left(\mathbf{x}-\mathbf{x}_{0}\right), \varphi(\mathbf{x})\right\rangle=\int_{R_{n}} \delta\left(\mathbf{x}-\mathbf{x}_{0}\right) \cdot \varphi(\mathbf{x}) \mathrm{d} \mathbf{x}=\varphi\left(\mathbf{x}_{0}\right) .
$$

Für den hier betrachteten dreidimensionalen Fall bedeutet das

$$
\begin{aligned}
\left\langle\delta\left(x-x_{0}\right)\right. & \left.\delta\left(y-y_{0}\right) \cdot \delta\left(z-z_{0}\right), \varphi(x, y, z)\right\rangle \\
& =\int_{-\infty}^{\infty} \int_{-\infty}^{\infty} \int_{-\infty}^{\infty} \delta\left(x-x_{0}\right) \cdot \delta\left(y-y_{0}\right) \cdot \delta\left(z-z_{0}\right) \cdot \varphi(x, y, z) \mathrm{d} x \mathrm{~d} y \mathrm{~d} z=\varphi\left(x_{0}, y_{0}, z_{0}\right) .
\end{aligned}
$$

Soll (B.10) gelten, dann muss also

$$
\begin{array}{r}
\lim _{t \rightarrow t_{0}} \int_{-\infty}^{\infty} \int_{-\infty}^{\infty} \int_{-\infty}^{\infty}\left(\frac{1}{2 \sqrt{\pi K\left(t-t_{0}\right)}}\right)^{3} \cdot \exp \left(-\frac{\left(x-x_{0}\right)^{2}+\left(y-y_{0}\right)^{2}+\left(z-z_{0}\right)^{2}}{4 K\left(t-t_{0}\right)}\right) \\
\times \varphi(x, y, z) \mathrm{d} x \mathrm{~d} y \mathrm{~d} z \stackrel{!}{=} \varphi\left(x_{0}, y_{0}, z_{0}\right)
\end{array}
$$

erfüllt sein. Substitution $\xi=1 /\left(t-t_{0}\right)$ liefert

$$
\begin{array}{r}
\lim _{\xi \rightarrow \infty} \int_{-\infty}^{\infty} \int_{-\infty}^{\infty} \int_{-\infty}^{\infty}\left(\frac{\sqrt{\xi}}{2 \sqrt{\pi K}}\right)^{3} \cdot \exp \left(-\frac{\xi \cdot\left(\left(x-x_{0}\right)^{2}+\left(y-y_{0}\right)^{2}+\left(z-z_{0}\right)^{2}\right)}{4 K}\right) \\
\times \varphi(x, y, z) \mathrm{d} x \mathrm{~d} y \mathrm{~d} z \stackrel{!}{=} \varphi\left(x_{0}, y_{0}, z_{0}\right)
\end{array}
$$

Weitere Substitutionen

$$
\begin{aligned}
& \tilde{x}=\sqrt{\xi} \cdot\left(x-x_{0}\right), \quad \text { also } \quad x=\frac{\tilde{x}}{\sqrt{\xi}}+x_{0} \quad \text { mit } \quad d x=\frac{1}{\sqrt{\xi}} d \tilde{x}, \\
& \tilde{y}=\sqrt{\xi} \cdot\left(y-y_{0}\right), \quad \text { also } \quad y=\frac{\tilde{y}}{\sqrt{\xi}}+y_{0} \quad \text { mit } \quad d y=\frac{1}{\sqrt{\xi}} d \tilde{x}, \\
& \tilde{z}=\sqrt{\xi} \cdot\left(z-z_{0}\right), \quad \text { also } \quad z=\frac{\tilde{z}}{\sqrt{\xi}}+z_{0} \quad \text { mit } \quad d z=\frac{1}{\sqrt{\xi}} d \tilde{x},
\end{aligned}
$$

\footnotetext{
${ }^{1}$ Das Integralzeichen steht hier nicht für ein Integral im Sinne der klassischen Analysis, sondern wird nur als Schreibweise verwendet [33].
} 
führen auf

$$
\begin{aligned}
& \int_{-\infty}^{\infty} \int_{-\infty}^{\infty} \int_{-\infty}^{\infty}\left(\frac{1}{2 \sqrt{\pi K}}\right)^{3} \cdot \exp \left(-\frac{\tilde{x}^{2}+\tilde{y}^{2}+\tilde{z}^{2}}{4 K}\right) \\
& \times \lim _{\xi \rightarrow \infty} \varphi\left(\frac{\tilde{x}}{\sqrt{\xi}}+x_{0}, \frac{\tilde{y}}{\sqrt{\xi}}+y_{0}, \frac{\tilde{z}}{\sqrt{\xi}}+z_{0}\right) \mathrm{d} \tilde{x} \mathrm{~d} \tilde{y} \mathrm{~d} \tilde{z} \stackrel{!}{=} \varphi\left(x_{0}, y_{0}, z_{0}\right)
\end{aligned}
$$

und weiter

$$
\int_{-\infty}^{\infty} \int_{-\infty}^{\infty} \int_{-\infty}^{\infty}\left(\frac{1}{2 \sqrt{\pi K}}\right)^{3} \cdot \exp \left(-\frac{\tilde{x}^{2}+\tilde{y}^{2}+\tilde{z}^{2}}{4 K}\right) \mathrm{d} \tilde{x} \mathrm{~d} \tilde{y} \mathrm{~d} \tilde{z} \cdot \varphi\left(x_{0}, y_{0}, z_{0}\right) \stackrel{!}{=} \varphi\left(x_{0}, y_{0}, z_{0}\right) .
$$

Bei dem Dreifachintegral in (B.18) handelt es sich um die Dichtefunktion der dreidimensionalen Normalverteilung [6], was durch die Substitution $\sigma=\sqrt{2 K}$ deutlich wird. Es besitzt den Wert Eins

$$
\int_{-\infty}^{\infty} \int_{-\infty}^{\infty} \int_{-\infty}^{\infty}\left(\frac{1}{\sigma \sqrt{2 \pi}}\right)^{3} \cdot \exp \left(-\frac{\tilde{x}^{2}+\tilde{y}^{2}+\tilde{z}^{2}}{2 \sigma^{2}}\right) \mathrm{d} \tilde{x} \mathrm{~d} \tilde{y} \mathrm{~d} \tilde{z}=1
$$

Die resultierende Forderung

$$
\varphi\left(x_{0}, y_{0}, z_{0}\right) \stackrel{!}{=} \varphi\left(x_{0}, y_{0}, z_{0}\right)
$$

ist damit erfüllt. Also ist (2.18) GreEnsche Funktion der partiellen Differenzialgleichung $(2.17)$. 


\section{C Übergang vom drei- zum zweidimensionalen Medium}

Gleichung (2.20)

$$
C_{G, 0}(\mathbf{x}, t)=\left(\frac{1}{2 \sqrt{\pi K t}}\right)^{n} \cdot \exp \left(-\frac{\left\|\mathbf{x}-\mathbf{x}_{0}\right\|_{2}^{2}}{4 K t}\right) \cdot 1(t)
$$

beschreibt die Greensche Funktion für Diffusion im $n$-dimensionalen Medium mit $t_{0}=0$. Nachfolgend wird gezeigt, dass die Greensche Funktion für $n=3$ in den Fall $n=2$ übergeht, wenn das dreidimensionale Medium durch zwei impermeable Flächen parallel zur $(z=0)$-Ebene begrenzt wird und der Abstand dieser Flächen gegen Null geht.

In Abschnitt 2.4.5 wird erläutert, dass der Einfluss impermeabler Flächen durch zusätzliche virtuelle Quellen modelliert werden kann, deren Positionen aus Spiegelungen an den Flächen resultieren. Die Lösung der Ausbreitungsgleichung ergibt sich durch Superposition der Konzentrationen der eigentlichen Quelle und aller virtuellen Quellen.

Für den Übergang wird hier ohne Einschränkung der Quellort bei $\mathbf{x}_{0}=\left(x_{0}, y_{0}, 0\right)^{T}$ gewählt. Zudem wird angenommen, dass die impermeablen Flächen bei $z= \pm L_{z} / 2$ liegen (Abb. C.1).

Die Greensche Funktion lautet damit für $z=0$ (vgl. (2.45))

$$
C_{G, 0, L_{z}}(\mathbf{x}, t)=\sum_{k=0}^{\infty} \frac{2}{(4 \pi K t)^{\frac{3}{2}}} \cdot \exp \left(-\frac{\left(x-x_{0}\right)^{2}+\left(y-y_{0}\right)^{2}+\left(-k L_{z}\right)^{2}}{4 K t}\right) \cdot 1(t) .
$$

Wegen der Symmetrie der impermeablen Flächen bezüglich des Quellorts kann sich im Unterschied $\mathrm{zu}$ (2.45) auf eine Summation von 0 bis $\infty$ beschränkt werden, wenn die Summe mit dem Faktor 2 multipliziert wird.

Für zweidimensionale Medien lautet die Greensche Funktion

$$
C_{G, 0}^{2 D}(\mathbf{x}, t)=\frac{1}{4 \pi K t} \cdot \exp \left(-\frac{\left(x-x_{0}\right)^{2}+\left(y-y_{0}\right)^{2}}{4 K t}\right) \cdot 1(t) .
$$




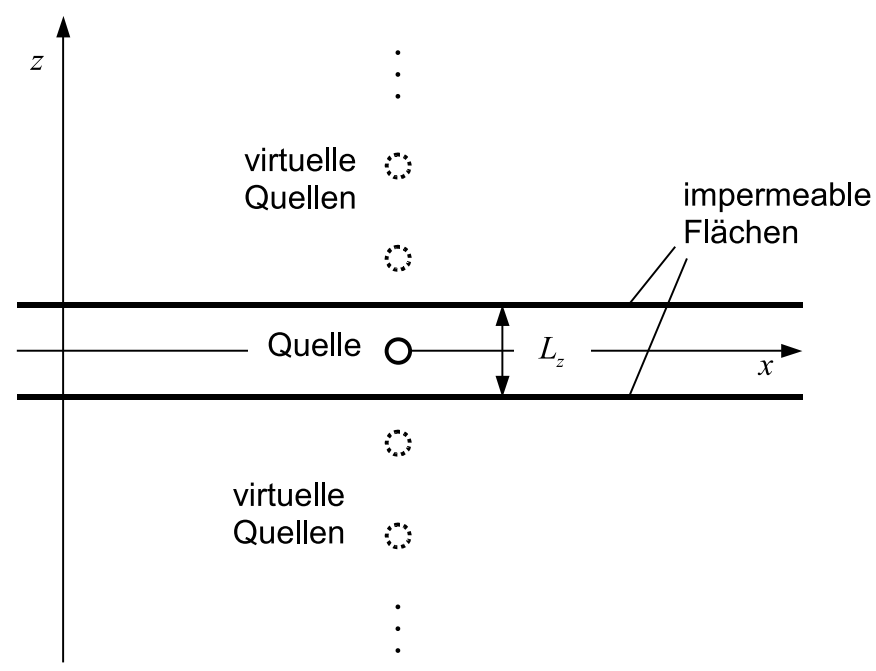

Abb. C.1: Lage der eigentlichen Quelle und der virtuellen Quellen im dreidimensionalen Medium mit zwei impermeablen Flächen parallel zur $x, y$-Ebene.

Diese Funktion liefert Konzentrationen mit der Einheit $\mathrm{mg} / \mathrm{m}^{2}$. Besitzt das Medium eine Ausdehnung $L_{z}$ in $z$-Richtung und wird ideale Durchmischung angenommen, ist durch $L_{z}$ $\mathrm{zu}$ dividieren, um Konzentrationen in der Einheit $\mathrm{mg} / \mathrm{m}^{3} \mathrm{zu}$ erhalten

$$
C_{G, 0, L_{z}}^{2 D}(\mathbf{x}, t)=\frac{1}{4 \pi K t} \cdot \exp \left(-\frac{\left(x-x_{0}\right)^{2}+\left(y-y_{0}\right)^{2}}{4 K t}\right) / L_{z} \cdot 1(t) .
$$

Für den Übergang vom drei- zum zweidimensionalen Medium wird der Grenzübergang $L_{z} \rightarrow 0$ gebildet. Für $L_{z} \rightarrow 0$ muss (C.2) gleich (C.4) sein. Um dies zu zeigen, wird (C.2) in

$$
C_{G, 0, L_{z}}(\mathbf{x}, t)=\frac{1}{4 \pi K t} \cdot \exp \left(-\frac{\left(x-x_{0}\right)^{2}+\left(y-y_{0}\right)^{2}}{4 K t}\right) \cdot 1(t) \cdot \sum_{k=0}^{\infty} \frac{2}{\sqrt{4 \pi K t}} \cdot \exp \left(-\frac{\left(k L_{z}\right)^{2}}{4 K t}\right)
$$

umgeformt. Aus (C.4) und (C.5) entsteht die Forderung

$$
\lim _{L_{z} \rightarrow 0} \sum_{k=0}^{\infty} \frac{2}{\sqrt{4 \pi K t}} \cdot \exp \left(-\frac{\left(k L_{z}\right)^{2}}{4 K t}\right) \cdot L_{z} \stackrel{!}{=} 1 .
$$

Mit der Definition für das bestimmte Riemannsche Integral zwischen den Grenzen $\xi=a$ und $\xi=b[14]$ gilt allgemein

$$
\lim _{L \rightarrow 0} \sum_{k=0}^{(b-a) / L-1} f(a+L \cdot k) \cdot L=\int_{a}^{b} f(\xi) \mathrm{d} \xi .
$$

Durch Anwendung dieser Beziehung lässt sich (C.6) als

$$
\int_{0}^{\infty} \frac{2}{\sqrt{4 \pi K t}} \cdot \exp \left(-\frac{\xi^{2}}{4 K t}\right) \mathrm{d} \xi \stackrel{!}{=} 1
$$


schreiben. Für die Bestimmung der oberen Integrationsgrenze wurde berücksichtigt, dass bei der Anwendung von (C.7) auf (C.6) die obere Summationsgrenze auch für $L \neq 0 \mathrm{zu}$ $\infty$ werden muss.

Die Substitution

$$
\chi=\frac{\xi}{4 K t} \quad \text { mit } \quad d \xi=\sqrt{4 K t} \mathrm{~d} \chi
$$

führt auf

$$
\frac{2}{\sqrt{\pi}} \int_{0}^{\infty} \mathrm{e}^{-\chi^{2}} \mathrm{~d} \chi \stackrel{!}{=} 1 .
$$

Die linke Seite ist aber gerade die Fehler-Funktion erf $(\infty)$, die den Wert 1 besitzt [14]. Damit ist die Forderung (C.6) erfüllt. Das bedeutet, dass sich der Übergang vom drei- zum zweidimensionalen Medium durch Einführen zweier impermeabler Flächen, die aufeinander zulaufen, erklären lässt. Für flache dreidimensionale Medien empfiehlt sich deshalbe eine Modellierung durch die zweidimensionale Ausbreitungsgleichung.

Der Übergang vom zwei- zum eindimensionalen Medium kann analog erfolgen. 


\section{Nachweis $\frac{\mathrm{dC}_{\mathrm{S}}(\mathrm{x}, t)}{\mathrm{d} t}=\mathrm{C}_{\mathrm{G}}(\mathrm{x}, t) \cdot q_{0}$}

Nachfolgend wird gezeigt, dass die zeitliche Ableitung von $C_{S}(\mathbf{x}, t)$ für die Quellfunktion $u(t)=q_{0} \cdot 1\left(t-t_{0}\right)$ identisch mit dem Produkt aus der zugehörigen GrEENschen Funktion $C_{G}(\mathbf{x}, t)$ mit der Quellfunktion $u(t)=\delta\left(t-t_{0}\right)$ und der konstanten Quellstärke $q_{0}$ ist.

Der Nachweis wird hier für den dreidimensionalen Fall geführt. Es ist also zu zeigen, dass die zeitliche Ableitung einer der Gleichungen (2.29) bis (2.31) gleich der GREEnschen Funktion (2.18) multipliziert mit $q_{0}$ ist. Mit (2.29) muss also

$$
\begin{array}{r}
\frac{\mathrm{d}}{\mathrm{d} t}\left[\frac{q_{0}}{8(\pi K)^{\frac{3}{2}}} \cdot \int_{t_{0}}^{t} \frac{1}{\left(t-t^{\prime}\right)^{\frac{3}{2}}} \cdot \exp \left(-\frac{\left\|\mathbf{x}-\mathbf{x}_{0}\right\|_{2}^{2}}{4 K\left(t-t^{\prime}\right)}\right) \mathrm{d} t^{\prime} \cdot 1\left(t-t_{0}\right)\right] \\
\stackrel{!}{=} \frac{1}{8\left(\pi K\left(t-t_{0}\right)\right)^{\frac{3}{2}}} \cdot \exp \left(-\frac{\left\|\mathbf{x}-\mathbf{x}_{0}\right\|_{2}^{2}}{4 K\left(t-t_{0}\right)}\right) \cdot 1\left(t-t_{0}\right) \cdot q_{0}
\end{array}
$$

gelten. Mit der Produktregel folgt für die linke Seite von (D.1)

$$
\begin{gathered}
\frac{\mathrm{d}}{\mathrm{d} t}\left[\frac{q_{0}}{8(\pi K)^{\frac{3}{2}}} \cdot \int_{t_{0}}^{t} \frac{1}{\left(t-t^{\prime}\right)^{\frac{3}{2}}} \cdot \exp \left(-\frac{\left\|\mathbf{x}-\mathbf{x}_{0}\right\|_{2}^{2}}{4 K\left(t-t^{\prime}\right)}\right) \mathrm{d} t^{\prime}\right] \cdot 1\left(t-t_{0}\right)+ \\
{\left[\frac{q_{0}}{8(\pi K)^{\frac{3}{2}}} \cdot \int_{t_{0}}^{t} \frac{1}{\left(t-t^{\prime}\right)^{\frac{3}{2}}} \cdot \exp \left(-\frac{\left\|\mathbf{x}-\mathbf{x}_{0}\right\|_{2}^{2}}{4 K\left(t-t^{\prime}\right)}\right) \mathrm{d} t^{\prime}\right] \cdot \delta\left(t-t_{0}\right) .}
\end{gathered}
$$

Der zweite Summand in (D.2) ist immer null, da

$$
\begin{gathered}
\delta\left(t-t_{0}\right)=0 \quad \text { für } t \neq t_{0} \quad \text { und } \\
\lim _{t \rightarrow t_{0}}\left[\frac{q_{0}}{8(\pi K)^{\frac{3}{2}}} \cdot \int_{t_{0}}^{t} \frac{1}{\left(t-t^{\prime}\right)^{\frac{3}{2}}} \cdot \exp \left(-\frac{\left\|\mathbf{x}-\mathbf{x}_{0}\right\|_{2}^{2}}{4 K\left(t-t^{\prime}\right)}\right) \mathrm{d} t^{\prime}\right]=0
\end{gathered}
$$

gelten. 
Für das zeitliche Ableiten des ersten Summanden von (D.2) wird die Beziehung

$$
\frac{\mathrm{d}}{\mathrm{d} t} \int_{\psi(t)}^{\varphi(t)} f\left(t^{\prime}, t\right) \mathrm{d} t^{\prime}=f(\varphi(t), t) \cdot \frac{\mathrm{d} \varphi(t)}{\mathrm{d} t}-f(\psi(t), t) \cdot \frac{\mathrm{d} \psi(t)}{\mathrm{d} t}+\int_{\psi(t)}^{\varphi(t)} \frac{\mathrm{d}}{\mathrm{d} t} f\left(t^{\prime}, t\right) \mathrm{d} t^{\prime}
$$

für das Ableiten eines bestimmten Integrals nach einem Parameter [39, S.44] verwendet.

Mit $\varphi(t)=t$ und $\psi(t)=t_{0}$ folgt für den linken Summanden von (D.2)

$$
\begin{array}{r}
{\left[\lim _{t^{\prime} \rightarrow t}\left(\frac{1}{\left(t-t^{\prime}\right)^{\frac{3}{2}}} \cdot \exp \left(-\frac{\left\|\mathbf{x}-\mathbf{x}_{0}\right\|_{2}^{2}}{4 K\left(t-t^{\prime}\right)}\right)\right)+\int_{t_{0}}^{t} \frac{\mathrm{d}}{\mathrm{d} t} \cdot \frac{1}{\left(t-t^{\prime}\right)^{\frac{3}{2}}} \cdot \exp \left(-\frac{\left\|\mathbf{x}-\mathbf{x}_{0}\right\|_{2}^{2}}{4 K\left(t-t^{\prime}\right)}\right) \mathrm{d} t^{\prime}\right]} \\
\times \frac{q_{0}}{8(\pi K)^{\frac{3}{2}}} \cdot 1\left(t-t_{0}\right) .
\end{array}
$$

Mit der Beziehung

$$
\int_{a}^{b} \frac{\mathrm{d}}{\mathrm{d} \xi} \cdot f(\xi-\sigma) \mathrm{d} \sigma=-f(\xi-b)+f(\xi-a)
$$

folgt für (D.6)

$$
\begin{array}{r}
{\left[\lim _{t^{\prime} \rightarrow t}\left(\frac{1}{\left(t-t^{\prime}\right)^{\frac{3}{2}}} \cdot \exp \left(-\frac{\left\|\mathbf{x}-\mathbf{x}_{0}\right\|_{2}^{2}}{4 K\left(t-t^{\prime}\right)}\right)\right)-\lim _{t^{\prime} \rightarrow t}\left(\frac{1}{\left(t-t^{\prime}\right)^{\frac{3}{2}}} \cdot \exp \left(-\frac{\left\|\mathbf{x}-\mathbf{x}_{0}\right\|_{2}^{2}}{4 K\left(t-t^{\prime}\right)}\right)\right)\right.} \\
\left.+\left(\frac{1}{\left(t-t_{0}\right)^{\frac{3}{2}}} \cdot \exp \left(-\frac{\left\|\mathbf{x}-\mathbf{x}_{0}\right\|_{2}^{2}}{4 K\left(t-t_{0}\right)}\right)\right)\right] \cdot \frac{q_{0}}{8(\pi K)^{\frac{3}{2}}} \cdot 1\left(t-t_{0}\right) .
\end{array}
$$

Es ist zu erkennen, dass sich die ersten beiden Terme gegeneinander aufheben und damit für die linke Seite von (D.1)

$$
\frac{q_{0}}{8\left(\pi K\left(t-t_{0}\right)\right)^{\frac{3}{2}}} \cdot \exp \left(-\frac{\left\|\mathbf{x}-\mathbf{x}_{0}\right\|_{2}^{2}}{4 K\left(t-t_{0}\right)}\right) \cdot 1\left(t-t_{0}\right)
$$

erhalten wird, was wie gefordert der rechten Seite entspricht. Damit gilt

$$
\frac{\mathrm{d} C_{S}(\mathbf{x}, t)}{\mathrm{d} t}=C_{G}(\mathbf{x}, t) \cdot q_{0}
$$




\section{Literaturverzeichnis}

[1] Adams, W. W.; Loustaunau, P.: An Introduction to Gröbner Bases. Providence, RI: Amer. Math. Soc, 1994.

[2] Alpay, M. E.: Model-based solution techniques for the source localization problem in distributed parameter systems. Dissertation, Oregon State University, 1999.

[3] Alpay, M. E.; Shor, M. H.: Model-based solution techniques for the source localization problem. IEEE Transactions on Control Systems Technology 8 (2000) 6, S. 893-902.

[4] Althainz, P.; Goschnick, J.; Ehrmann, S.; Ache, H.: Multisensor microsystem for contaminants in air. Sensors and Actuators B: Chemical 33 (1996), S. 72 76.

[5] Arnold, C.; Harms, M.; Goschnick, J.: Air Quality Monitoring And Fire Detection With The Karlsruhe Electronic Micronose KAMINA. IEEE Sensors Journal 2 (2002) 3, S. 179-188.

[6] BAndemer, H.; NÄTheR, W.: Theorie und Anwendung der optimalen Versuchsplanung, Bd. 2. Berlin: Akademie-Verlag, 1980.

[7] Bargon, J.; Braschoss, S.; Flörke, J.; Herrmann, U.; Klein, L.; LoerGen, J. W.; Lopez, M.; Maric, S.; Parham, A. H.; et Al., P. P.: Determination of the ripening state of Emmental cheese via quartz microbalances. Sensors and Actuators B: Chemical 95 (2003) 1-3, S. 6-19.

[8] Barisci, J. N.; Wallace, G. G.; Andrews, M. K.; Partridge, A. C.; HarRIS, P. D.: Conducting polymer sensors for monitoring aromatic hydrocarbons using an electronic nose. Sensors and Actuators B: Chemical 84 (2002) 2-3, S. 252-257.

[9] Beck, J.; Cole, K. D.; Haji-Sheikh, A.; Litkouhi, B.: Heat conduction using Green's functions. London, Washington DC, Philadelphia: Hemisphere publishing corporation, 1982.

[10] Ben-Israel, A.; Greville, T. N. E.: Generalized inverses: theory and applications. New York, London, Sydney, Toronto: John Wiley and Sons, 1974. 
[11] Bender, F.; Barié, N.; Romoudis, G.; Voigt, A.; Rapp, M.: Developement of a preconcentration unit for a SAW sensor micro array and its use for indoor air quality monitoring. Sensors and Actuators B: Chemical 93 (2003) 1-3, S. 135-141.

[12] Bicego, M.; Tessari, G.; Tecchiolli, G.; Bettinelli, M.: A comparative analysis of basic pattern recognition techniques for the development of small size electronic nose. Sensors and Actuators B: Chemical 85 (2002) 1-2, S. 137-144.

[13] Bronstein, I. N.; Semendjajew, K. A.: Taschenbuch der Mathematik. Leipzig: Verlag Nauka, Moskau, BSB B. G. Teubner Verlagsgesellschaft, 1983.

[14] Bronstein, I. N.; Semendjajew, K. A.; Musiol, G.; Mühlig, H.: Taschenbuch der Mathematik. Thun, Frankfurt a. M.: Harri Deutsch, 1999.

[15] Buchberger, B.: Multidimensional Systems Theory. Kap. 6 "Gröbner Bases: An Algorithmic Method in Polynomial Ideal Theory", New York: van Nostrand Reinhold, 1982.

[16] Capone, S.; Epifani, M.; Quaranta, F.; Siciliano, P.; Taurino, A.; VasaNELLI, L.: Monitoring of rancidity of milk by means of an electronic nose and a dynamic PCA analysis. Sensors and Actuators B: Chemical 78 (2001) 1-3, S. 174179.

[17] Carmel, L.; Sever, N.; Lancet, D.; Harel, D.: An eNose algorithm for identifying chemicals and determining their concentration. Sensors and Actuators B: Chemical 93 (2003) 1-3, S. 77-83.

[18] Carslaw, H. S.; Jaeger, J. C.: Conduction of heat in solids. Oxford: Clarendon Press, 1959.

[19] Churchill, R. V.: Operational Mathematics. New York: McGraw-Hill Book Company, 3. Aufl., 1972.

[20] Corless, R. M.; Gonnet, G. H.; Hare, D. E. G.; Jeffrey, D. J.; Knuth, D. E.: On the Lambert W function. Adv. Comput. Math. 5 (1996), S. 329-359.

[21] Crank, J.: The mathematics of diffusion. Oxford: Clarendon Press, 1975.

[22] Cussler, E. L.: Diffusion - Mass transfer in fluid systems. Cambridge: Cambridge University Press, 1984.

[23] Di Ruscio, D.: A weighted view on the partial least-squares algorithm. Automatica 36 (2000), S. 831-850.

[24] Duckett, T.; Axelsson, M.; Saffiotti, A.: Learning to locate an odour source with a mobile robot. In: IEEE International Conference on Robotics and Automation, S. 4017-4021, Seoul, Korea, 2001. 
[25] Ehrmann, S.; Jüngst, J.; Goschnick, J.; Everhard, D.: Application of a gas sensor microarray to human breath analysis. Sensors and Actuators B: Chemical 65 (2000) 1-3, S. 247-249.

[26] Freijer, J. I.; Veling, E. J. M.; Hassanizadeh, S. M.: Analytical solutions of the convection-dispersion equation applied to transport of pesticides in soil columns. Environmental Modelling and Software 13 (1997) 1, S. 139-149.

[27] Frietsch, M.; Zudock, F.; Goschnick, J.; Bruns, M.: CuO catalytic membrane as selectivity trimmer for metal oxide gas sensors. Sensors and Actuators B: Chemical 65 (2000) 1-3, S. 379-381.

[28] Gardner, J. W.: An introduction to electronic nose technology. Stanstead, UK: Neotronics Scientific Ltd., 1996.

[29] Gardner, J. W.; Bartlett, P. N. (Hg.): Sensors and sensory systems for an electronic nose, International Series of Numerical Mathematics, Dordrecht, Netherlands: Kluwer Academic Publishers, 1992.

[30] Gardner, J. W.; Bartlett, P. N.: A brief history of electronic noses. Sensors and Actuators B: Chemical 18 (1994) 1-3, S. 210-211.

[31] Gardner, J. W.; Shin, H. W.; Hines, E. L.: An electronic nose system to diagnose illness. Sensors and Actuators B: Chemical 70 (2000) 1-3, S. 19-24.

[32] Garrigues, S.; Talou, T.; Nesa, D.; Gaset, A.: Modified GC/MS system versus dedicated MS device: comparative application of electronic nose for QC in automotive industry. Sensors and Actuators B: Chemical 78 (2001) 1-3, S. 337-344.

[33] Gelfand, I. M.; Shilov, G. E.: Verallgemeinerte Funktionen (Distributionen). Berlin: VEB Deutscher Verlag der Wissenschaften, 1960.

[34] Goschnick, J.: An electronic nose for intelligent consumer products based on a gas analytical gradient microarray. Microelectronic Engineering 57-58 (2001), S. 693704.

[35] Goschnick, J.: Application trends, technical requirements and ojectives of further development for electronic noses. In: 203rd Meeting of the Electrochemical Society, Paris, 2003.

[36] Goschnick, J.; Arnold, C.; Blank, T.; Eppler, W.; Häringer, D.; Hartmann, V.; Keller, H. B.; Matthes, J.; Reiling, V.: Air Monitoring in Buildings with Networked Electronic Noses. Oral presentation at 10th International Symposium on Olfaction and Electronic Noses (ISOEN 03), 2003. 
[37] Goschnick, J.; WAlter, T.: ELMINA - Elektronische Mikronasen für Überwachungs- und Regelaufgaben in Gebäuden und Produktionsanlagen. Abschlussbericht des HGF-Strategieprojektes. Wissenschaftliche Berichte, Forschungszentrum Karlsruhe, 2003.

[38] Gouws, G. J.; Gouws, D. J.: Analyte identification using concentration modulation and wavelet analysis of QCM sensors. Sensors and Actuators B: Chemical 91 (2003) 1-3, S. 326-332.

[39] Gradstein, I. S.; Ryshik, I. M.: Summen-, Produkt- und Integraltafeln, Bd. 1 und 2. Thun, Frankfurt a. M.: Harri Deutsch, 1981.

[40] Groetsch, C. W.: Inverse Problems in the Mathematical Sciences. Braunschweig, Wiesbaden: Vieweg, 1993.

[41] GRÖLL, L.: LS-Identifikation mit einer quadratischen Restriktion. In: GMA-FA 1.4 Theoretische Verfahren der Regelungstechnik, S. 172-180, Johannes Kepler Universität Linz, 2002.

[42] Gröll, L.: Least Squares with a Single Quadratic Constrained. at - Automatisierungstechnik 52 (2004) 1, S. 48-55.

[43] Guadarrama, A.; Rodríguez-Méndez, M. L.; de Saja, J. A.: Conducting polymer-based array for the discrimination of odours from trim plastic materials used in automobiles. Analytica Chimica Acta 455 (2002) 1, S. 41-47.

[44] Hamacher, T.; Niess, J.; Lammers, P. S.; Diekmann, B.; Boeker, P.: Online measurement of odorous gases close to the odour threshold with a QMB sensor system with an integrated preconcentration unit. Sensors and Actuators B: Chemical 95 (2003) 1-3, S. 39-45.

[45] Hänsel, H.: Grundzüge der Fehlerrechnung. Berlin: VEB Deutscher Verlag der Wissenschaften, 1965.

[46] Harris, P. D.; Arnold, W. M.; Andrews, M. K.; Partridge, A. C.: Resistance characteristics of conducting polymer films used in gas sensors. Sensors and Actuators B: Chemical 42 (1997) 3, S. 177-184.

[47] Haugen, J. E.; KvaAl, K.: Electronic nose and artificial neural network. Meat Science 49 (1998) 1, S. 273-286.

[48] Hayes, A. T.; Martinoli, A.; Goodman, R. M.: Distributed odor source localization. IEEE Sensors Journal 2 (2002) 3, S. 260-271.

[49] Haykin, S.: Neural networks - A comprehensive foundation. New Jersey: Prentice Hall Inc., 2. Aufl., 1999. 
[50] Hill, J. M.; Dewynne, J. N.: Heat conduction. Oxford, London, Edinburgh, Boston, Palo Alto, Melbourne: Blackwell Scientific Publications, 1987.

[51] Ishida, H.; Kagawa, Y.; Nakamoto, T.; Moriızumi, T.: Odor source localization in the clean room by an autonomous mobile sensing system. Sensors and Actuators B: Chemical 33 (1996), S. 115-121.

[52] Ishida, H.; Nakamoto, T.; Moriızumi, T.; Kikas, T.; Janata, J.: Plumetracking robots: A new application of chemical sensors. Biological Bulletin 200 (2001), S. 222-226.

[53] JAKов, W.: HyGLEAM - An Approach to Generally Applicable Hybridization of Evolutionary Algorithms. In: Parallel Problem Solving from Nature : 7th International Conference (Guervos, J. J. M.; Adamidis, P.; Beyer, H. G.; FernandezVillacanas, J. L.; Schwefel, H. P., Hg.), S. 527-36, 2002.

[54] Jeremić, A.; Nehorai, A.: Design of chemical sensor arrays for monitoring disposal sites on the ocean floor. IEEE J. Oceanic Eng. 23 (1998), S. 334-343.

[55] Jeremić, A.; Nehorai, A.: Landmine detection and localization using chemical sensor array processing. IEEE Transactions on Signal Processing 48 (2000), S. 12951305 .

[56] Jerger, A.; Kohler, H.; Becker, F.; Keller, H. B.; Seifert, R.: Intelligent sensor system for reliable monitoring of ammonia leakages. In: 8th International Meeting on Chemical Sensors, Basel, 2000.

[57] Jerger, A.; Kohler, H.; Becker, F.; Keller, H. B.; Seifert, R.: New applications of tin oxide gas sensors II. Intelligent sensor system for reliable monitoring of ammonia leakages. Sensors and Actuators B: Chemical 81 (2002) 2-3, S. 301-307.

[58] Kersten, M.; Matthes, J.; Fouda, C.; Zipser, S.; Keller, H. B.: Customizing UML for the Development of Distributed Reactive Systems and Ada 95 Code Generation. Ada User Journal 23 (2002), S. 175-182.

[59] Khapalov, A. Y.: Localization of unknown sources for parabolic systems on the basis of available observations. Int. Journal Systems Sci. 25 (1994) 8, S. 1305-1322.

[60] Kinzelbach, W.: Numerische Methoden zur Modellierung des Transports von Schadstoffen im Grundwasser, Bd. 21 von Schriftenreihe Gwf Wasser - Abwasser. München, Wien: Oldenbourg Verlag, 1987.

[61] Kinzelbach, W.; Rausch, R.: Grundwassermodellierung - Eine Einführung mit Übungen. Stuttgart, Berlin: Borntraeger, 1995. 
[62] LAI, Z.: Acuisition and analysis of measurement data from spatially distributed electronic noses: the example of building monitoring. Diplomarbeit, FH Karlsruhe, 2004

[63] LeE, D.-D.; LeE, D.-S.: Environmental Gas Sensors. IEEE Sensors Journal 1 (2001) 3, S. 214-224.

[64] Lilienthal, A.; Zell, A.; Wandel, M.; Weimar, U.: Sensing odour sources in indoor environments without a constant airflow by a mobile robot. In: IEEE International Conference on Robotics and Automation, S. 4005-4009, Seoul, South Korea, 2000.

[65] Lilienthal, A.; Zell, A.; Wandel, M.; Weimar, U.: Detektion und Lokalisation einer Geruchsquelle mit einem Autonomen Mobilen Roboter. In: VDI-Berichte 1679, S. 689-694, VDI/VDE-Gesellschaft Mess- und Automatisierungstechnik, 2002.

[66] Lin, Y.; Guo, H.; Chang, Y.; KaO, M.; Wang, H.; Hong, R.: Application of the electronic nose for uremia diagnosis. Sensors and Actuators B: Chemical 76 (2001) 1-3, S. 177-180.

[67] Linder, R.; PÖPPL, S. J.: A new neural network approach classifies olfactory signals with high accuracy. Quality and Preference 14 (2003) 5-6, S. 435-440.

[68] Lunn, M.; Lunn, R. J.; MackAy, R.: Determining analytical solutions of multiple species contaminant transport, with sorption and decay. Journal of Hydrology 180 (1996) 1, S. 195-210.

[69] Martens, R.; Massmeyer, K.; Pfeffer, W.; Haider, G.; Morlock, G.: Bestandsaufnahme und Bewertung der derzeit genutzten atmosphärischen Ausbreitungsmodelle. Köln: Gesellschaft für Reaktorsicherheit, 1987.

[70] Matthes, J.: Lokalisierung von Emissionsquellen mit ortsfesten, räumlich verteilten elektronischen Nasen. In: GMA-Kongress 2003, S. 495-502, Baden-Baden, 2003.

[71] Matthes, J.; Gröll, L.: Model based source localisation by distributed sensors for point sources and diffusion. In: 13th IFAC Symposium on System Identification., S. 319-324, Rotterdam, The Netherlands, 2003.

[72] Matthes, J.; Gröll, L.; Keller, H. B.: Source localization by spatially distributed electronic noses for advection and diffusion. IEEE Trans. on Signal Proc. (2003), (in press).

[73] Matthes, J.; Gröll, L.; Keller, H. B.: Source localization by stationary, spatially distributed electronic noses. Sensors and Actuators A: Physical (2004), (in press). 
[74] Matthes, J.; Keller, H. B.; Heker, W. D.; Kersten, M.; Fouda, F.: Zuverlässige Software durch den Einsatz von UML, MDA und der Programmiersprache Ada. In: Informatik 2003: Mit Sicherheit Informatik; Beiträge des Schwerpunktes Sicherheit - Schutz und Zuverlässigkeit, Lecture Notes in Informatics; P36, S. 167178, Bonn: Gesellschaft für Informatik, 2003.

[75] Menzel, R.; Goschnick, J.: Gradient gas sensor microarrays for on-line process control, a new dynamic classification model for fast and reliable air quality assessment. Sensors and Actuators B: Chemical 68 (2000) 1-3, S. 115-122.

[76] Meyer, E.; Neumann, E. G.: Physikalische und Technische Akustik. Vieweg Verlag, 1979.

[77] Mielle, P.: Electronic noses: Towards the objective instrumental characterization of food aroma. Trends in Food Science and Technology 7 (1996) 12, S. 432-438.

[78] Morvan, M.; Talou, T.; Beziau, J.: MOS-MOSFET gas sensors array measurements versus sensory and chemical characterisation of VOC's emissions from car seat foams. Sensors and Actuators B: Chemical 95 (2003) 1-3, S. 212-223.

[79] Morvan, M.; Talou, T.; Gaset, A.; Beziau, J. F.: Electronic-nose systems for control quality applications in automotive industry. Sensors and Actuators B: Chemical 69 (2000) 3, S. 384-388.

[80] Nakamoto, T.; Fukunishi, K.; Morimzumi, T.: Perfume and flavour identification by odour-sensing system using quartz-resonator sensor array and neural-network pattern recognition. Sensors and Actuators B: Chemical 10 (1993) 2, S. 351-354.

[81] Nambo, H.; Kimura, H.; Oyabu, T.: Estimation of gas generation point using delay of gas sensor responses. IEEJ Transactions on Sensors and Micromachines 122 (2002) 10, S. 480-486.

[82] Natale, C. D.; Macagnano, A.; Martinelli, E.; Paolesse, R.; D’Arcangelo, G.; Roscioni, C.; Finazzi-Agrò, A.; D’Amico, A.: Lung cancer identification by the analysis of breath by means of an array of non-selective gas sensors. Biosensors and Bioelectronics 18 (2003) 10, S. 1209-1218.

[83] Nehorai, A.; Porat, B.; PAldi, E.: Detection and localization of vapor-emitting sources. IEEE Transactions on Signal Processing 43 (1995) 1, S. 243-253.

[84] Nievergelt, Y.: Solution to an inverse problem in diffusion. SIAM Rev. 40 (1998) 1, S. $74-80$.

[85] Nitz, S.; Kollmannsberger, H.; Lachermeier, C.; Horner, G.: Odour Assessment with Piezoelectric Quartz Crystal Sensor Arrays, a Suitable Tool for Quality Control in Food Technology. Adv. Food Sci. 21 (1999) 1, S. 136-150. 
[86] O’Connell, M.; Valdora, G.; Peltzer, G.; Negri, R.: A practical approach for fish freshness determinations using a portable electronic nose. Sensors and Actuators B: Chemical 80 (2001) 2, S. 81-87.

[87] Penza, M.; Cassano, G.: Application of principal component analysis and artificial neural networks to recognize the individual VOCs of methanol/2-propanol in a binary mixture by SAW multi-sensor array. Sensors and Actuators B: Chemical 89 (2003) 3, S. 269-284.

[88] Pierce, A. D.: Acoustics, An introduction to its principles and applications. Acoustical Society of America, 1991.

[89] Ping, W.; Jun, X.: A novel recognition method for electronic nose using artificial neural network and fuzzy recognition. Sensors and Actuators B: Chemical 37 (1996) 3, S. 169-174.

[90] RaO, C. R.; Mitra, S. K.: Generalized inverse of matrices and its applications. New York, London, Sydney, Toronto: John Wiley and Sons, 1971.

[91] RApp, M.; Bender, F.; Voigt, A.: A novel SAW micro array concept for environmental organic gas detection at low concentrations. In: 1st IEEE Internat.Conf. On Sensors, Orlando, 2002.

[92] Rapp, M.; Reibel, J.: Gasanalytik mit Sensorsystemen: Ein Weg zur elektronischen Nase? Nachr. Chem. Tech. Lab. 44 (1996), S. 1088-1092.

[93] Reibel, J.; Stahl, U.; Wessa, T.; Rapp, M.: Gas analysis with SAW sensor systems. Sensors and Actuators B: Chemical 65 (2000) 1-3, S. 173-175.

[94] Roberts, O. F. T.: The theoretical scattering of smoke in a turbulent atmosphere. In: Proceedings of the Royal Society of London, Bd. 104, S. 640-654, 1923.

[95] Russell, R. A.: Odor detection by mobile robots. Singapore: World Scientific, 1999.

[96] Russell, R. A.; Thiel, D.; Deveza, R.; Mackay-Sim, A.: A robotic system to locate hazardous chemical leaks. In: IEEE International Conference on Robotics and Automation, S. 556-561, Nagoya, 1995.

[97] Schädler, G.; Bächlin, W.; Lohmeyer, A.; Wees, T. V.: Vergleich und Bewertung derzeit verfügbarer mikroskaliger Strömungs- und Ausbreitungsmodelle. In: Berichte Umweltforschung Baden-Württemberg (FZKA-PEF 138), 418, Forschungszentrum Karlsruhe, 1996.

[98] Schieberle, P.; Freiling, A.; Bock, J.; Heinert, L.; Kohl, D.; Krummel, C.; Hofmann, T.: High resolution gas chromatography/selective odorant measurement by multisensor array (HRGC/SOMSA): A useful approach to standardise 
multisensor arrays for use in the detection of key food odorants. Sensors and Actuators B: Chemical 41 (1997) 1-3, S. 81-87.

[99] SchröDer, K.: Mathematik für die Praxis. Frankfurt/M., Zürich: Verlag Harri Deutsch, 1964.

[100] Shaffer, R. E.; Rose-Pehrsson, S. L.; McGill, R. A.: Multiway analysis of preconcentrator sample surface acoustic wave chemical sensor array data. Field Analytical Chemistry and Technology 2 (1998) 3, S. 179-192.

[101] Shilov, G. E.: Generalized functions and partial differential equations. New York, London, Paris: Gordon and Breach Science Publishers, Inc., 1968.

[102] Sminnow, W. I.: Lehrgang der höheren Mathematik, Bd. 4. Berlin: VEB Deutscher Verlag der Wissenschaften, 1963.

[103] Snopok, B. A.; Kruglenko, I. V.: Multisensor systems for chemical analysis: State-of-the-art in electronic nose technology and new trends in machine olfaction. Thin Film Solids 418 (2002) 1, S. 21-41.

[104] Söderström, T.; Stoica, P.: System identification. New York, London, Toronto, Sydney, Tokyo: Prentice Hall, 1989.

[105] Sohn, J. H.; Smith, R.; Yoong, E.; Leis, J.; Galvin, G.: Quantification of Odours from Piggery Effluent Ponds using an Electronic Nose and an Artificial Neural Network. Biosystems Engineering 86 (2003) 4, S. 399-410.

[106] Srivastava, A. K.: Detection of volatile organic compounds (VOCs) using SnO2 gas-sensor array and artificial neural network. Sensors and Actuators B: Chemical 96 (2003) 1-2, S. 24-37.

[107] Sun, Y.; Petersen, J. N.; Clement, T. P.: Analytical solutions for multiple species reactive transport in multiple dimensions. Journal of Contaminant Hydrology 35 (1999) 1, S. 429-440.

[108] Taurino, A.; Capone, S.; Distante, C.; Epifani, M.; Rella, R.; Siciliano, $\mathrm{P}$.: Recognition of olive oils by means of an integrated sol-gel $\mathrm{SnO} 2$ Electronic Nose. Food Quality and Preference 13 (2002) 6, S. 81-87.

[109] Ulmer, H.; Mitrovics, J.; Noetzel, G.; Weimar, U.; Göpel, W.: Odours and flavours identified with hybrid modular sensor systems. Sensors and Actuators B: Chemical 43 (1997) 1-3, S. 24-33.

[110] Vaid, T. P.; Lewis, N. S.: The use of 'electronic nose' sensor responses to predict the inhibition activity of alcohols on the cytochrome P-450 catalyzed phydroxylation of aniline. Bioorganic and Medicinal Chemistry 8 (2000) 4, S. 795805. 
[111] VeIt, I.: Technische Akustik. Vogel Verlag, 1996.

[112] Wold, H.: Encyclopedia of statistics sciences, Bd. 6, Kap. Partial least squares, S. 581-591. New York: Wiley, 1985.

[113] YAnG, Y.; YANG, P.; WANG, X.: Electronic nose based on SAWS array and its odor identification capability. Sensors and Actuators B: Chemical 66 (2000) 1-3, S. $167-170$.

[114] Young, R. C.; Buttner, W. J.; Linnell, B. R.; Ramesham, R.: Electronic nose for space program applications. Sensors and Actuators B: Chemical 93 (2003) 1-3, S. 7-16.

[115] Zenger, A.: Atmosphärische Ausbreitungsmodellierung: Grundlagen und Praxis. Berlin, Heidelberg, New York: Springer Verlag, 1998.

[116] Zwillinger, D.: Handbook of differential equations. Boston, San Diego, New York, Berkeley, London, Sydney, Tokyo, Toronto: Academic Press, Inc., 1989. 